\title{
Entwicklung eines faltbaren Werkstoffverbundes auf Furnierbasis
}

\section{Dissertation}

\author{
zur Erlangung des Doktorgrades \\ „Dr. rer. nat." \\ der Fakultät für Forstwissenschaften und Waldökologie \\ der Georg-August-Universität Göttingen \\ vorgelegt von \\ Timo Ackermann \\ geboren am 21.01.1984 in Limburg a. d. Lahn, Hessen
}

Göttingen im März 2020 
1. Gutachter: Prof. Dr. Alireza Kharazipour, Abt. Molekulare Holzbiotechnologie und Technische Mykologie, AG Chemie und Verfahrenstechnik für Verbundwerkstoffe, BüsgenInstitut, Georg-August-Universität Göttingen

2. Gutachter: $\quad$ Prof. Dr. Carsten Mai, Abt. Holzbiologie und Holztechnologie, Burckhardt-Institut, Georg-August-Universität Göttingen 
Veröffentlichte Schriften, die in Zusammenhang mit dieser Dissertation stehen:

Ackermann, Timo (2103): Faltbare Werkstoffverbünde - Neuartige Materialverbindungen zur Herstellung hochwertiger, flexibel anwendungsbezogener Verpackungen, Displays und Behältnisse. Forschungsbericht 2013 der Hochschule Rosenheim, S. 16-17.

Im Rahmen des Promotionsvorhabens fanden u. a. zwei Abschlussarbeiten statt. Die ermittelten Rohdaten aus den Arbeiten:

Werndl, Peter (2016): Entwicklung eines biobasierten, faltbaren Verbundwerkstoffes unter Berücksichtigung spezieller technologischer Eigenschaften. Masterarbeit an der Fakultät für Holztechnik der Hochschule Rosenheim

und

Werndl, Peter (2014): Untersuchungen ausgewählter Materialeigenschaften eines neuartigen, faltbaren Verbundwerkstoffes. Bachelorarbeit an der Fakultät für Holztechnik der Hochschule Rosenheim

wurden auch in den Kapitel 4.2, 4.3.4 und 4.5.2 verwendet. 


\section{Danksagung}

Die vorliegende Arbeit wurde vom Verfasser als externem Promotionsstudent am Büsgen-Institut der Georg-August-Universität Göttingen, AG Chemie und Verfahrenstechnik von Verbundwerkstoffen erstellt.

Das Projekt und damit auch die praktische Umsetzung fand in der Abteilung Forschung und Entwicklung der Hochschule Rosenheim in Kooperation mit der Firma Josef Wochner GmbH \& Co. KG, Rosenfeld Heiligenzimmern statt.

Mein besonderer Dank gilt daher meinem Doktorvater Herrn Prof. Dr. Alireza Kharazipour für die aufgrund der großen Distanz nicht immer einfachen Betreuung und Unterstützung während meines Promotionsvorhabens.

Zu großem Dank bin ich auch Herrn Prof. Dr. Matthias Zscheile verpflichtet, der mich nicht nur an die Hochschule Rosenheim holte, das äußerst spannende Thema stellte, sondern mich stets auch in allen beruflichen Belangen gefördert und unterstützt hat.

Herrn Prof. Dr. Carsten Mai danke ich ganz herzlich für seine stets lösungsorientierte und unkomplizierte Betreuung im Rahmen des Promotionsstudiums sowie für die Übernahme der Zweitgutachtertätigkeit.

Ein weiterer Dank gilt Herrn PD. Dr. Markus Euring für sein stets offenes Ohr und seine Hilfestellung.

Mein ganz besonders großer Dank gilt Frau Dr. Nina Ritter, die mich während der vielen Jahre stets mit vollem Einsatz unterstützte, motivierte und in die richtigen Bahnen lenkte.

Herrn Wolfgang Wochner möchte ich an dieser Stelle herzlich für die freundschaftliche Zusammenarbeit und seine stetige Unterstützung während der gemeinsamen Projektjahre und darüber hinaus danken.

Ein weiterer Dank gilt dem Team der Abteilung Forschung und Entwicklung der Technischen Hochschule Rosenheim für die schöne gemeinsame Zeit. Herrn Wolfgang Alversammer und Herrn Peter Werndl sei an dieser Stelle besonders gedankt.

Und natürlich gilt der größte Dank meiner Familie und meinen Freunden die mich über die vielen Jahre stets unterstützt, gestärkt und ermutigt haben. Dies gilt besonders für meine liebe Ehefrau und meine kleine Tochter, die aufgrund dieser Arbeit in ihren ersten Lebenswochen häufig auf ihren Vater verzichten musste. Daher widme ich diese Arbeit meiner Tochter.

Vielen Dank! 


\section{Inhaltsverzeichnis}

I. INHALTSVERZEICHNIS. .. $\mathrm{I}$

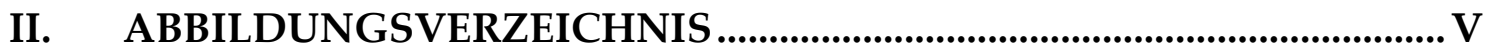

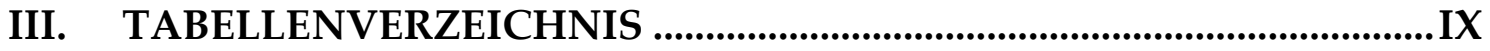

IV. ABKÜRZUNGSVERZEICHNIS .........................................................XIII

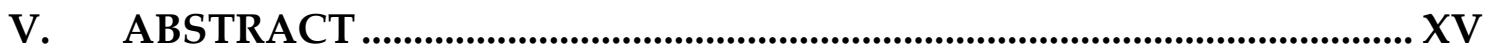

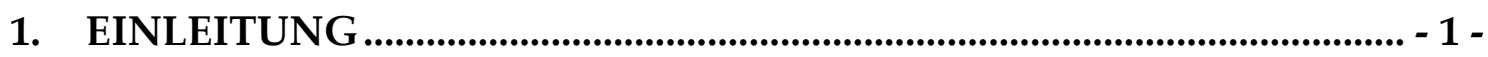

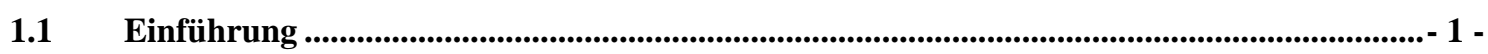

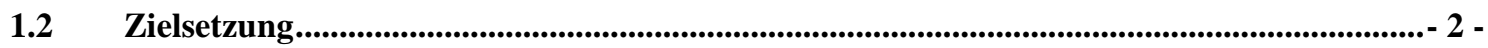

2. THEORETISCHE GRUNDLAGEN ...................................................... - 3 -

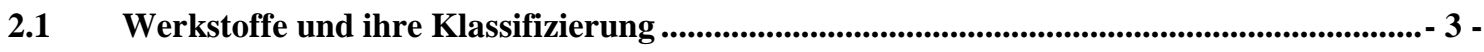

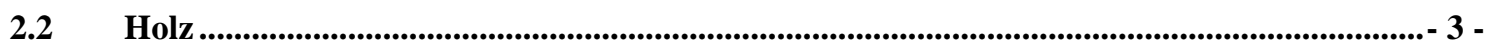

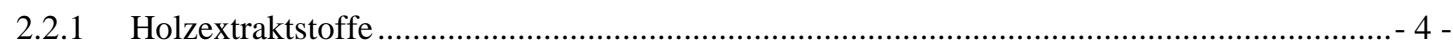

2.2.1.1 Die Bedeutung von Holzextraktstoffen in technologischer Hinsicht ............................ 4 -

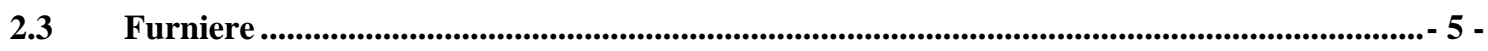

2.3.1 Grundlegendes zu Furnieren ………………………………………………………. 5 -

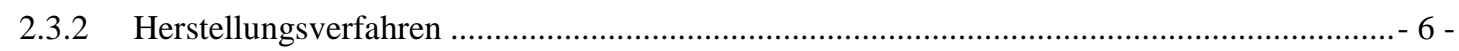

2.3.3 Furniere und ihre wirtschaftliche Bedeutung …………...................................................... 8 -

2.4 Polyvinylchlorid .............................................................................................................................. 9 -

2.4.1 Herstellungsverfahren von PVC .......................................................................... 9 -

2.4.2 PVC und seine wirtschaftliche Bedeutung ......................................................... 11 -

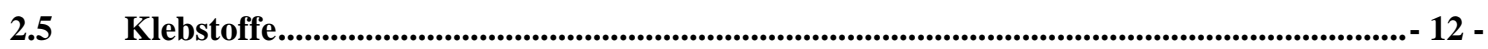


2.5.1 Aufbau von Klebstoffen ................................................................................ 12 -

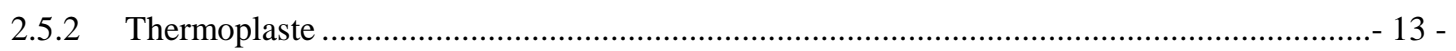

2.5.3 Duromere .................................................................................................... 13 -

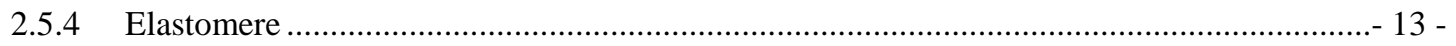

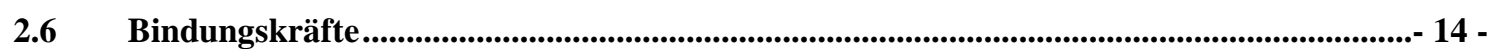

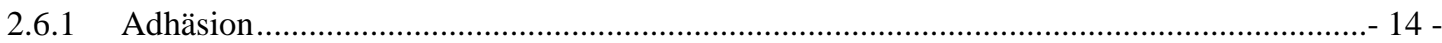

2.6.2 Kohäsion...................................................................................................... 15 -

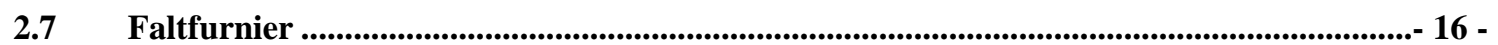

2.7.1 Aufbau von Faltfurnier ........................................................................................ 16 -

3. MATERIAL UND METHODEN_............................................................. - 18 -

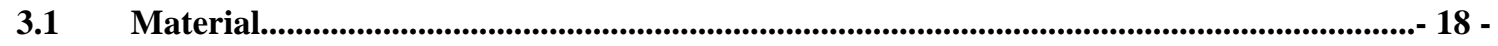

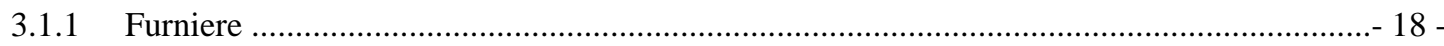

3.1.2 Material für die Mittelschicht .................................................................................... 19 -

3.1.3 Klebstoffe ........................................................................................................... 19

3.1.3.1 Furnierleim auf Polyvinylacetatbasis ............................................................ 19 -

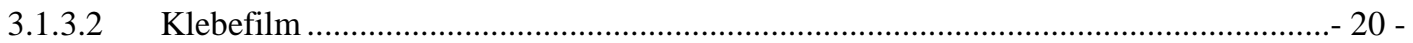

3.1.3.3 Cyanacrylatklebstoff .................................................................................... 20 -

3.1.4 Technische Geräte zur Herstellung von Faltfurnierplatten ............................................... 20 -

3.1.5 Analytische Messgeräte …................................................................................... 21 -

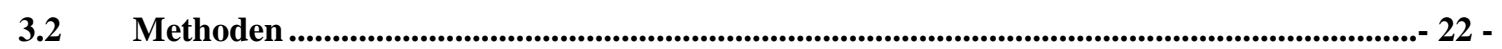

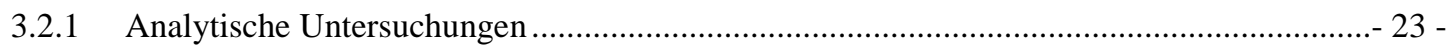

3.2.1.1 Bestimmung des Extraktstoffgehaltes in Kaltwasser ........................................... 23 -

3.2.1.2 Bestimmung des Extraktstoffgehaltes in Heißwasser .............................................. 24 -

3.2.1.3 Bestimmung des Extraktstoffgehaltes mittels sukzessiver Extraktion ...................... 24 -

3.2.1.4 Bestimmung der Oberflächenrauigkeit ...................................................................- 25 -

3.2.2 Bestimmung der mechanisch technologischen Eigenschaften...................................... 26 -

3.2.2.1 Normvorgaben zur Prüfung von Faltfurnier .................................................... 26 -

3.2.2.2 Vorabprüfung mittels Prüfmuster ...................................................................... 26 -

3.2.2.3 Probenahme, Zuschnitt und Überwachung (Deutsche Norm DIN EN 326-1)............- 27 - 


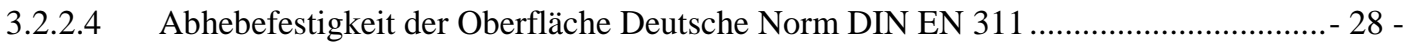

3.2.2.5 Bestimmung des Biege-Elastizitätsmoduls und der Biegefestigkeit (DIN EN 310)....- 29 -

3.2.2.6 Bestimmung der Zugeigenschaften Deutsche Norm DIN EN ISO 527-3 ................. 29 -

3.2.2.7 Modifizierte Bestimmung der Zugspannung ....................................................... 30 -

3.2.2.8 Festigkeits- und Dauerhaftigkeitsuntersuchungen der Faltnut in Anlehnung an

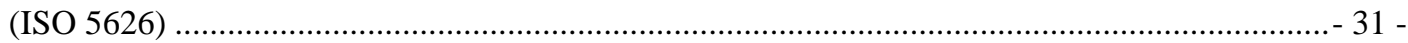

3.2.2.9 Lagerung im starken Wechselklima ...................................................................... 32 -

3.2.3 Herstellung des Verbundwerkstoffes .................................................................... 33 -

3.2.3.1 Aufbau des Verbundwerkstoffes ......................................................................... 33 -

3.2.3.2 Herstellungsprozess ................................................................................. $34-$

3.2.4 Statistische Auswertung der Daten......................................................................... 35 -

4. ERGEBNISSE UND DISKUSSION .......................................................... - 37 -

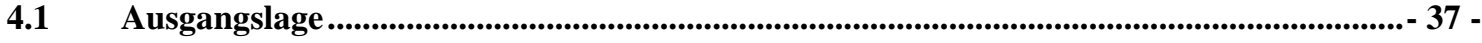

4.2 Werkstoffuntersuchungen der PVC-Folie ....................................................................... 39 -

4.2.1 Untersuchung der Zugfestigkeit unter Berücksichtigung der Produktionsrichtung.............. 39 -

4.2.2 Untersuchung der Zugfestigkeit bei verschiedenen Ausgangsbedingungen.......................- 42 -

4.2.3 Untersuchung der Zugfestigkeit in verschiedenen Winkeln .............................................. 44 -

4.3 Untersuchung von Abhebefestigkeiten verschiedener Werkstoffverbünde auf der Basis von Erlen- und Eichenfurnieren .................................................................................................................. - 49 -

4.3.1 Untersuchung der Abhebefestigkeit von Erlenfurnier auf PVC-Folie............................... 49 -

4.3.2 Untersuchung der Abhebefestigkeit von Eichenfurnier auf PVC-Folie ............................- 63 -

4.3.3 Vergleiche der Abhebefestigkeit zwischen Erlenfurnier und Eichenfurnier auf PVC-Folie - 77 -

4.3.4 Untersuchungen der Abhebefestigkeit von mittels PVAc-Leim verklebten Erlenfurnieren.- 82 -

4.3.5 Untersuchungen der Abhebefestigkeit von mittels thermoplastischem Klebefilm verklebten

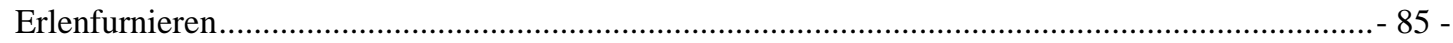

4.3.6 Vergleichende Diskussion der Erlenfurnierverklebung unter Verwendung von PVAc-Leim und thermoplastischem Klebefilm $-91-$

4.4 Vergleichende analytische Untersuchungen von Erlen- und Eichenfurnieren.................. 93 -

4.4.1 Vergleichende Untersuchung der Extraktstoffgehalte von Erlen- und Eichenfurnier .......... 93 - 
4.4.1.1 Heißwasser- und Kaltwasserextraktion sowie sukzessive Extraktion von Erlen- und

Eichenfurnier.....

$-93-$

4.4.2 Vergleiche der Oberflächenrauigkeit von Erlen- und Eichenfurnier ................................ 98 -

4.5 Werkstoffuntersuchungen des Gesamtverbundes/Halbzeugs ...........................................- 100 -

4.5.1 Biegefestigkeitsprüfung des Verbundwerkstoffes aus Erlenfurnier auf PVC-Folie ...........- 101 -

4.5.2 Dauerhaftigkeitsuntersuchungen der Faltnut ............................................................. 106 -

4.5.3 Lagerung des Verbundwerkstoffes in starkem Wechselklima........................................- 108 -

5. ZUSAMMENFASSUNG ………………........................................... 110 -

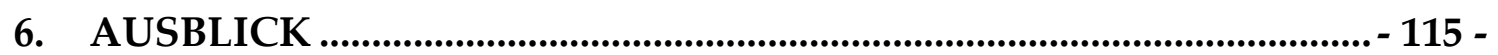

7. LITERATURVERZEICHNIS ………………………….................................. - 117 -

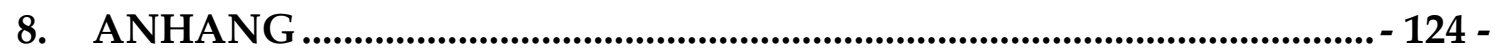

EIDESSTATTLICHE ERKLÄRUNG …........................................................ - 126 - 


\section{Abbildungsverzeichnis}

ABBILDUNG 2-1: MARKTENTWICKLUNG FURNIER IN DEUTSCHLAND 2008-2016 IN MIO. EURO NACH (VDM 2017)............................................................................................ 8 -

ABBILDUNG 2-2: PVC VERARBEITUNG NACH PRODUKTGRUPPEN IM JAHR 2013 (DATEN NACH (CONSULTIC 2014B)) - 11 -

ABBILDUNG 2-3: KLEBSTOFFEINTEILUNG NACH DEM ABBINDEMECHANISMUS (HABENICHT 2009). -12 -

ABBILDUNG 2-4: NAHAUFNAHME EINER FALTFURNIERPLATTE ZUR DARSTELLUNG

DES 5-SCHICHTIGEN AUFBAUS - 16 ABBILDUNG 2-5: SEITLICHE AUFSICHT AUF EINE BEIDSEITIG EINGEFRÄSTE FALTFURNIERPLATTE. HIER MIT DURCH DEN FRÄSKOPF BESCHÄDIGTER MITTELSCHICHT (FOTO: P. WERNDL). $17-$ ABBILDUNG 3-1: MICRO PROF MIT ERLENFURNIERPROBE BESTÜCKT. 1- OPTISCHER MESSKOPF, 2- PROBEKÖRPER AUS ERLENFURNIER UND 3- BEWEGLICHER PRÜFTISCH. $25-$ ABBILDUNG 3-2: INTERESSENSBEREICH UND FAHRWEG DES MESSKOPFES FÜR DIE BESTIMMUNG DER OBERFLÄCHENRAUIGKEIT DER VERWENDETEN FURNIERE....$25-$

ABBILDUNG 3-3: PRÜFMUSTER ZUR ERSTEN KONTROLLE DER ADHÄSIVEN UND KOHÄSIVEN EIGENSCHAFTEN DES MATERIALVERBUNDES. $-26-$ ABBILDUNG 3-4: FOTOGRAFIE DER OBERFLÄCHE DER PVC-FOLIE BEI 10-FACHER VERGRÖßERUNG MITTELS AUFLICHTMIKROSKOP. STRUKTURIERTE SEITE (LINKS) UND UNSTRUKTURIERTE SEIT (RECHTS). (AUFNAHME: PETER WERNDL)...........- 27 ABBILDUNG 3-5: EINSPANNVORRICHTUNG ZUR ZUGSPANNUNGSPRÜFUNG IM $90^{\circ}$ WINKEL (LINKS) UND $45^{\circ}$ WINKEL (RECHTS) MONTIERT AUF EINER UNIVERSALPRÜFMASCHINE. (FOTO: PETER WERNDL) -30 ABBILDUNG 3-6: TÜRPRÜFMASCHINE MIT EIGENS ANGEFERTIGTER PRÜFVORRICHTUNG (FOTO: PETER WERNDL) ............................................................... 31 ABBILDUNG 3-7: SCHEMATISCHE DARSTELLUNG DES GENERELLEN AUFBAUS DES VERBUNDWERKSTOFFES. $-33-$ ABBILDUNG 3-8: SCHEMATISCHE DARSTELLUNG DES HERSTELLUNGSPROZESSES DES VERBUNDWERKSTOFFES. - 34 - 
ABBILDUNG 3-9: SCHEMATISCHE DARSTELLUNG DES ABLAUFES DER STATISTISCHEN DATENAUSWERTUNG. $-35-$

ABBILDUNG 3-10: AUFBAU DER IN DIESER ARBEIT VERWENDETEN BOXPLOTS ZUR DARSTELLUNG DER STATISTISCHEN LAGE- UND STREUPARAMETER. $-36-$ ABBILDUNG 4-1: BOXPLOTS ZU DEN VERSUCHSERGEBNISSEN DER ZUGFESTIGKEITSPRÜFUNGEN PARALLEL UND SENKRECHT ZUR EXTRUSIONSRICHTUNG. -40 ABBILDUNG 4-2: FLASCHENTRÄGER AUS FALTFURNIER, BEI DESSEN FERTIGUNG DIE EXTRUSIONSRICHTUNG BEACHTET WERDEN SOLLTE. -41 ABBILDUNG 4-3: BOXPLOTS DER ERMITTELTEN ZUGFESTIGKEITEN ZU DEN MESSREIHEN „NORM“ ${ }^{\prime \prime}, 45^{\circ}$ “ UND , $90^{\circ}$. $-47-$ ABBILDUNG 4-4: IDEALES FRÄSBILD (LINKS), FEHLERHAFTE FRÄSNUTEN MIT VERSATZ UND ZU HOHER EINRITZTIEFE (RECHTS) (FOTO: P. WERNDL)..............- 48 ABBILDUNG 4-5: STARK ASYMMETRISCHER VERSATZ DER FRÄSNUTEN. (FOTO: P. WERNDL). $-48-$ ABBILDUNG 4-6: BEISPIEL FÜR EINE FEHLERHAFTE ABHEBEFESTIGKEITSPRÜFUNG. DAS FURNIER WURDE DURCH DEN PRÜFPILZ NICHT FLÄCHIG ABGELÖST UND DAS VERSUCHSERGEBNIS SOMIT VERWORFEN. $-51-$ ABBILDUNG 4-7: BOXPLOTS ZUR ABHEBEFESTIGKEIT VON ERLENFURNIER AUF PVC BEI 60 SEKUNDEN PRESSZEIT, SORTIERT NACH GESAMTERGEBNIS SOWIE DER PROBENLAGE INNERHALB DER PLATTEN. $-52-$ ABBILDUNG 4-8: BOXPLOTS ZUR ABHEBEFESTIGKEIT VON ERLENFURNIER AUF PVC BEI 90 SEKUNDEN PRESSZEIT, SORTIERT NACH GESAMTERGEBNIS SOWIE DER PROBENLAGE INNERHALB DER PLATTEN. $-54-$ ABBILDUNG 4-9: BOXPLOTS ZUR ABHEBEFESTIGKEIT VON ERLENFURNIER AUF PVC BEI 120 SEKUNDEN PRESSZEIT, SORTIERT NACH GESAMTERGEBNIS SOWIE DER PROBENLAGE INNERHALB DER PLATTEN. $-55-$ ABBILDUNG 4-10: BOXPLOTS ZUR ABHEBEFESTIGKEIT VON ERLENFURNIER AUF PVC BEI 180 SEKUNDEN PRESSZEIT, SORTIERT NACH GESAMTERGEBNIS SOWIE DER PROBENLAGE INNERHALB DER PLATTEN. $-57-$ ABBILDUNG 4-11: BOXPLOTS ZUR ABHEBEFESTIGKEIT VON ERLENFURNIER AUF PVC BEI 240 SEKUNDEN PRESSZEIT, SORTIERT NACH GESAMTERGEBNIS SOWIE DER PROBENLAGE INNERHALB DER PLATTEN. $-58-$ 
ABBILDUNG 4-12: ENTWICKLUNG DER ABHEBEFESTIGKEIT VON ERLENFURNIER AUF PVC IN RELATION ZU DEN PRESSZEITEN. $60-$

ABBILDUNG 4-13: VERGLEICHENDE BOXPLOTS ZUR ABHEBEFESTIGKEIT VON ERLENFURNIER AUF PVC, SORTIERT NACH DEN JEWEILIGEN PRESSZEITEN....- 61 ABBILDUNG 4-14: BOXPLOTS ZUR ABHEBEFESTIGKEIT VON EICHENFURNIER AUF PVC BEI 60 SEKUNDEN PRESSZEIT, SORTIERT NACH GESAMTERGEBNIS SOWIE DER PROBENLAGE INNERHALB DER PLATTEN. $65-$

ABBILDUNG 4-15: BOXPLOTS ZUR ABHEBEFESTIGKEIT VON EICHENFURNIER AUF PVC BEI 90 SEKUNDEN PRESSZEIT, SORTIERT NACH GESAMTERGEBNIS SOWIE DER PROBENLAGE INNERHALB DER PLATTEN. $-67-$

ABBILDUNG 4-16: BOXPLOTS ZUR ABHEBEFESTIGKEIT VON EICHENFURNIER AUF PVC BEI 120 SEKUNDEN PRESSZEIT, SORTIERT NACH GESAMTERGEBNIS SOWIE DER PROBENLAGE INNERHALB DER PLATTEN. $68-$

ABBILDUNG 4-17: BOXPLOTS ZUR ABHEBEFESTIGKEIT VON EICHEFURNIER AUF PVC BEI 180 SEKUNDEN PRESSZEIT, SORTIERT NACH GESAMTERGEBNIS SOWIE DER PROBENLAGE INNERHALB DER PLATTEN. $-70-$

ABBILDUNG 4-18: BOXPLOTS ZUR ABHEBEFESTIGKEIT VON EICHENFURNIER AUF PVC BEI 240 SEKUNDEN PRESSZEIT, SORTIERT NACH GESAMTERGEBNIS SOWIE DER PROBENLAGE INNERHALB DER PLATTEN. $-71-$ ABBILDUNG 4-19: ENTWICKLUNG DER ABHEBEFESTIGKEIT VON EICHENFURNIER AUF PVC IN RELATION ZU DEN PRESSZEITEN. $-73-$ ABBILDUNG 4-20: VERGLEICHENDE BOXPLOTS ZUR ABHEBEFESTIGKEIT VON EICHENFURNIER AUF PVC, SORTIERT NACH DEN JEWEILIGEN PRESSZEITEN. - 74 ABBILDUNG 4-21: ENTWICKLUNG DER ABHEBEFESTIGKEIT VON ERLEN- UND EICHENFURNIER AUF PVC-FOLIE, VERKLEBT MIT THERMOPLASTISCHEM KLEBEFILM, IN RELATION ZU DEN PRESSZEITEN. $-77-$ ABBILDUNG 4-22: BOXPLOTS ZU DEN ABHEBEFESTIGKEITSPRÜFUNGEN VON ERLENUND EICHENFURNIER AUF PVC-FOLIE, VERKLEBT MIT THERMOPLASTISCHEM KLEBEFILM, BEI VERSCHIEDENEN PRESSZEITEN. - 79 ABBILDUNG 4-23: BOXPLOTS ZUR ABHEBEFESTIGKEIT VON ERLENFURNIER AUF ERLENFURNIER VERKLEBT MIT „KLEIBERIT 322“ BEI 120 SEKUNDEN PRESSZEIT, SORTIERT NACH GESAMTERGEBNIS SOWIE DER PROBENLAGE INNERHALB DER PLATTEN. - 84 - 
ABBILDUNG 4-24: BOXPLOTS ZUR ABHEBEFESTIGKEIT VON ERLENFURNIER AUF ERLENFURNIER VERKLEBT MIT „COLLANO 46.002“ BEI 120 SEKUNDEN PRESSZEIT, SORTIERT NACH GESAMTERGEBNIS SOWIE DER PROBENLAGE INNERHALB DER PLATTEN. $-88-$

ABBILDUNG 4-25: TEMPERATURVERLAUF ÜBER DIE PRESSZEIT BEI ERLENFURNIER AUF PVC, GEMESSEN IN DEN KLEBEFUGEN ZWISCHEN FURNIER UND PVC AUF DER UNTER- UND OBERSEITE $-89-$

ABBILDUNG 4-26: DARSTELLUNG DER MITTELS „MICROPROF" AUFGENOMMENEN OBERFLÄCHENTOPOGRAPHIE VON ERLENFURNIER. -98 ABBILDUNG 4-27: DARSTELLUNG DER MITTELS „MICROPROF" AUFGENOMMENEN OBERFLÄCHENTOPOGRAPHIE VON EICHENFURNIER -99 ABBILDUNG 4-28: BOXPLOTS ZUR BIEGEFESTIGKEIT DES GESAMTVERBUNDES AUS ERLENFURNIEREN AUF PVC-FOLIE, SORTIERT NACH DER FASERAUSRICHTUNG DES DECKFURNIERS ZUM PRÜFSTEMPEL $102-$ ABBILDUNG 4-29: BOXPLOTS ZUM BIEGE-E-MODUL DES GESAMTVERBUNDES AUS ERLENFURNIEREN AUF PVC-FOLIE, SORTIERT NACH DER FASERAUSRICHTUNG DES DECKFURNIERS ZUM PRÜFSTEMPEL.................................................................- 103 ABBILDUNG 4-30: SERIENGRAFIK EINER BIEGEFESTIGKEITSVERSUCHSREIHE VON FALTFURNIER. DIE UNTEREN KURVEN GEHÖREN ZUR VERSUCHSREIHE „PARALLEL“ DIE OBEREN ZU „SENKRECHT“ $106-$ ABBILDUNG 4-31: FOTO DER FALTFURNIERPLATTE MIT DEN AUFFÄLLIGSTEN VERÄNDERUNGEN NACH DER LAGERUNG IM WECHSELKLIMA $109-$ 


\section{Tabellenverzeichnis}

TABELLE 2-1: KENNZEICHNENDE EIGENSCHAFTEN VON E-, S-, UND M-PVC IN ANLEHNUNG AN (KAISER 2007),...............................................................................- 10 -

TABELLE 3-1: EXKTRAKTIONSSTUFEN UND ZUGEHÖRIGES LÖSUNGSMITTEL............- 24 TABELLE 3-2: ÜBERSICHT ÜBER DIE LAGE UND ANZAHL DER PROBEKÖRPER JE VERSUCH. $-28-$

TABELLE 3-3: PRÜFPARAMETER FÜR DAUERHAFTIGKEITSVERSUCHE...........................- 32 -

TABELLE 3-4: SOLL-KLIMA WÄHREND DER VERSUCHSPERIODE.......................................... 32 TABELLE 4-1: ERGEBNISSE DER ZUGFESTIGKEITSPRÜFUNG IN UNTERSCHIEDEN LAGEN ZUR EXTRUSIONSRICHTUNG $-39-$

TABELLE 4-2: ERGEBNISSE DER ZUGFESTIGKEITSPRÜFUNG DER PVC-FOLIE IN ANLIEFERUNGS- UND TEMPERIERTEM ZUSTAND. $42-$

TABELLE 4-3: STATISTISCHE ERGEBNISSE DER ZUGVERSUCHE AN PVC-FOLIE IM ANGEWINKELTEN ZUSTAND. $-44-$

TABELLE 4-4: VERGLEICHENDE DARSTELLUNG DER MESSWERTE DER VERSUCHSREIHEN "NORM", "45" UND "90" $46-$ TABELLE 4-5: HERSTELLUNGSPARAMETER DES UNTERSUCHTEN MATERIALVERBUNDS AUS ERLE AUF PVC. $50-$ TABELLE 4-6: ERGEBNISSE DER ABHEBEFESTIGKEITSPRÜFUNG VON ERLENFURNIER AUF PVC BEI 60 SEKUNDEN PRESSZEIT, DIFFERENZIERT NACH DEM GESAMTERGEBNIS UND DEN JEWEILIGEN LAGEN DER PROBEKÖRPER IN DEN PROBEPLATTEN. -52 -

TABELLE 4-7: ERGEBNISSE DER ABHEBEFESTIGKEITSPRÜFUNG VON ERLENFURNIER AUF PVC BEI 90 SEKUNDEN PRESSZEIT, DIFFERENZIERT NACH DEM GESAMTERGEBNIS UND DEN JEWEILIGEN LAGEN DER PROBEKÖRPER IN DEN PROBEPLATTEN. $-53-$

TABELLE 4-8: ERGEBNISSE DER ABHEBEFESTIGKEITSPRÜFUNG VON ERLENFURNIER AUF PVC BEI 120 SEKUNDEN PRESSZEIT, DIFFERENZIERT NACH DEM GESAMTERGEBNIS UND DEN JEWEILIGEN LAGEN DER PROBEKÖRPER IN DEN PROBEPLATTEN. - 55 -

TABELLE 4-9: ERGEBNISSE DER ABHEBEFESTIGKEITSPRÜFUNG VON ERLENFURNIER AUF PVC BEI 180 SEKUNDEN PRESSZEIT, DIFFERENZIERT NACH DEM 
GESAMTERGEBNIS UND DEN JEWEILIGEN LAGEN DER PROBEKÖRPER IN DEN PROBEPLATTEN. $-56-$

TABELLE 4-10: ERGEBNISSE DER ABHEBEFESTIGKEITSPRÜFUNG VON ERLENFURNIER AUF PVC BEI 240 SEKUNDEN PRESSZEIT, DIFFERENZIERT NACH DEM GESAMTERGEBNIS UND DEN JEWEILIGEN LAGEN DER PROBEKÖRPER IN DEN PROBEPLATTEN. $-58-$

TABELLE 4-11: VERGLEICHENDE ERGEBNISSE DER ABHEBEFESTIGKEITSPRÜFUNGEN VON ERLENFURNIER AUF PVC BEI VERSCHIEDENEN PRESSZEITEN. $-59-$

TABELLE 4-12: HERSTELLUNGSPARAMETER DES UNTERSUCHTEN MATERIALVERBUNDS AUS EICHENFURNIER AUF PVC-FOLIE. $-63-$

TABELLE 4-13: ERGEBNISSE DER ABHEBEFESTIGKEITSPRÜFUNG VON EICHENFURNIER AUF PVC BEI 60 SEKUNDEN PRESSZEIT, DIFFERENZIERT NACH DEM GESAMTERGEBNIS UND DEN JEWEILIGEN LAGEN DER PROBEKÖRPER IN DEN PROBEPLATTEN. $64-$

TABELLE 4-14: ERGEBNISSE DER ABHEBEFESTIGKEITSPRÜFUNG VON EICHENFURNIER AUF PVC BEI 90 SEKUNDEN PRESSZEIT, DIFFERENZIERT NACH DEM GESAMTERGEBNIS UND DEN JEWEILIGEN LAGEN DER PROBEKÖRPER IN DEN PROBEPLATTEN. $-66-$

TABELLE 4-15: ERGEBNISSE DER ABHEBEFESTIGKEITSPRÜFUNG VON EICHENFURNIER AUF PVC BEI 120 SEKUNDEN PRESSZEIT, DIFFERENZIERT NACH DEM GESAMTERGEBNIS UND DEN JEWEILIGEN LAGEN DER PROBEKÖRPER IN DEN PROBEPLATTEN $-67-$

TABELLE 4-16: ERGEBNISSE DER ABHEBEFESTIGKEITSPRÜFUNG VON EICHENFURNIER AUF PVC BEI 180 SEKUNDEN PRESSZEIT, DIFFERENZIERT NACH DEM GESAMTERGEBNIS UND DEN JEWEILIGEN LAGEN DER PROBEKÖRPER IN DEN PROBEPLATTEN. 69

TABELLE 4-17: ERGEBNISSE DER ABHEBEFESTIGKEITSPRÜFUNG VON EICHENFURNIER AUF PVC BEI 240 SEKUNDEN PRESSZEIT, DIFFERENZIERT NACH DEM GESAMTERGEBNIS UND DEN JEWEILIGEN LAGEN DER PROBEKÖRPER IN DEN PROBEPLATTEN. $-71-$

TABELLE 4-18: VERGLEICHENDE ERGEBNISSE DER ABHEBEFESTIGKEITSPRÜFUNGEN VON EICHENFURNIER AUF PVC BEI VERSCHIEDENEN PRESSZEITEN. $-72-$ 
TABELLE 4-19: VERGLEICHENDE ERGEBNISSE DER ABHEBEFESTIGKEITSPRÜFUNGEN VON ERLENFURNIER AUF PVC-FOLIE, VERKLEBT MIT THERMOPLASTISCHEM KLEBEFILM, BEI VERSCHIEDENEN PRESSZEITEN. $78-$

TABELLE 4-20: VERGLEICHENDE ERGEBNISSE DER ABHEBEFESTIGKEITSPRÜFUNGEN VON EICHENFURNIER AUF PVC-FOLIE, VERKLEBT MIT THERMOPLASTISCHEM KLEBEFILM, BEI VERSCHIEDENEN PRESSZEITEN. $-78-$

TABELLE 4-21: HERSTELLUNGSPARAMETER DES UNTERSUCHTEN FURNIERPLATTEN AUS ERLENFURNIER AUF ERLENFURNIER VERKLEBT MITTELS „KLEIBERIT 322“....82 -

TABELLE 4-22: ERGEBNISSE DER ABHEBEFESTIGKEITSPRÜFUNG VON ERLENFURNIER AUF ERLENFURNIER VERKLEBT MIT „KLEIBERIT 322“ BEI 120 SEKUNDEN PRESSZEIT, DIFFERENZIERT NACH DEM GESAMTERGEBNIS UND DEN JEWEILIGEN LAGEN DER PROBEKÖRPER IN DEN PROBEPLATTEN. $83-$

TABELLE 4-23: HERSTELLUNGSPARAMETER DER UNTERSUCHTEN FURNIERPLATTEN AUS ERLENFURNIER AUF ERLENFURNIER VERKLEBT MITTELS „COLLANO 46.002“. -86 -

TABELLE 4-24: ERGEBNISSE DER ABHEBEFESTIGKEITSPRÜFUNG VON ERLENFURNIER AUF ERLENFURNIER VERKLEBT MIT „COLLANO 46.002“ BEI 120 SEKUNDEN PRESSZEIT, DIFFERENZIERT NACH DEM GESAMTERGEBNIS UND DEN JEWEILIGEN LAGEN DER PROBEKÖRPER IN DEN PROBEPLATTEN $-87-$

TABELLE 4-25: VERGLEICHENDE ERGEBNISSE DER ABHEBEFESTIGKEITSPRÜFUNG VON ERLENFURNIER AUF ERLENFURNIER VERKLEBT MIT „PVAC“ UND „KLEBEFILM“. 91 -

TABELLE 4-26: MITTELWERTE DER HEIß- UND KALTWASSEREXTRAKTE VON ERLENUND EICHENFURNIER UNTER BERÜCKSICHTIGUNG DER ZUGEHÖRIGEN PHWERTE UND ALKALISCHEN PUFFERKAPAZITÄTEN. -94 -

TABELLE 4-27: NACH LÖSUNGSMITTELN SORTIERTE, MITTLERE EXTRAKTSTOFFGEHALTE NACH SUKZESSIVER EXTRAKTION VON ERLEN- UND EICHENFURNIER. - 95 -

TABELLE 4-28: EXKTRAKTIONSMITTEL DER SUKZESSIVEN EXKTRAKTION MIT ENTSPRECHENDEN STOFFKLASSEN IM EXTRAKT (NACH FAIX 2012). - 97 -

TABELLE 4-29: HERSTELLUNGSPARAMETER DER FALTFURNIERPLATTEN AUS ERLENFURNIEREN UND PVC-FOLIE ZUR HERSTELLUNG DER PRÜFKÖRPER FÜR DIE BIEGEFESTIGKEITSPRÜFUNG -101 - 
TABELLE 4-30: VERGLEICHENDE ERGEBNISSE DER BIEGEFESTIGKEITSPRÜFUNGEN DES GESAMTVERBUNDES SOWIE SORTIERUNG DER ERGEBNISSE NACH AUSRICHTUNG DER HOLZFASER DES DECKFURNIERS. $-102-$

TABELLE 4-31: VERGLEICHENDE WERTE VON FALTFURNIERPLATTEN ZU ANDEREN HOLZBASIERTEN WERKSTOFFEN IN BEZUG AUF DIE BIEGEFESTIGKEIT UND DEN BIEGE-E-MODUL (WERTE FÜR DIE WERKSTOFFE ENTNOMMEN AUS (NIEMZ 2002A; NIEMZ UND SONDEREGGER 2017)) ................................................................... 104 -

TABELLE 4-32: ERGEBNISSE DER DAUERHAFTIGKEITSPRÜFUNG， IN BEZUG AUF MAXIMAL MÖGLICHE FALTINTERVALLE, DES GESAMTVERBUNDES AUS ERLE MIT PVC-FOLIE. $107-$

TABELLE 4-33: ÜBERSICHT ZUR LAGERZEIT, DER TEMPERATUR, UND DER LUFTFEUCHTIGKEIT DER KLIMAKAMMER SOWIE DEN SICH ÄNDERNDEN GEWICHTEN DER PROBEPLATTEN. $109-$ 


\section{Abkürzungsverzeichnis}

\begin{tabular}{|c|c|c|c|}
\hline$\circ$ & Grad (Winkelmaß) & MV 2 & Materialverbund 2 \\
\hline${ }^{\circ} \mathrm{C}$ & Grad Celcius & $\mathrm{N}$ & Newton \\
\hline Abb. & Abbildung & $\mathrm{NaOH}$ & Natriumhydroxid \\
\hline atro & absolut trocken & o. g. & oben genannt \\
\hline bzw. & beziehungsweise & OSB & Oriented strand board \\
\hline $\mathrm{CO}_{2}$ & Kohlenstoffdioxid & $\mathrm{pH}$ & $\begin{array}{l}\text { Negativ dekadischer Logarithmus } \\
\text { der Protonenkonzentration }\end{array}$ \\
\hline DIN & $\begin{array}{l}\text { Deutsches Institut für Normung } \\
\text { e.V. }\end{array}$ & PK & Prüfkörper \\
\hline EN & Europäische Norm & PP & Polypropylen \\
\hline EPDM & Ethylen-Propylen-Dien-Kautschuk & $\%$ & Prozent \\
\hline etc. & et cetera & PVAc & Polyvinylacetat \\
\hline Fa. & Firma & PVC & Polyvinylchlorid \\
\hline g & Gramm & rpm & $\begin{array}{l}\text { revolutions per minute } \\
\text { (Umdrehung pro Minute) }\end{array}$ \\
\hline h & Stunde & $\mathrm{s}$ & Sekunde \\
\hline $\mathrm{HDH}$ & $\begin{array}{l}\text { Hauptverband der Deutschen } \\
\text { Holzindustrie und Kunststoffe } \\
\text { verarbeitenden Industrie und } \\
\text { verwandter Industrie- und } \\
\text { Wirtschaftszweige e.V. }\end{array}$ & $S$ & Standardabweichung \\
\hline ISO & $\begin{array}{l}\text { International Organization for } \\
\text { Standardization }\end{array}$ & s. g. & so genannt \\
\hline $\mathrm{kN}$ & Kilonewton & $\mathrm{t}$ & Tonne(n) \\
\hline kt & Kilotonne(n) & t/a & Tonne(n) pro Jahr \\
\hline M & Molarität & u. a. & unter anderem \\
\hline$\mu \mathrm{m}$ & Mikrometer & u. $\ddot{A}$. & und Ähnliche \\
\hline MDF & Mitteldichte Holzfaserplatte & $\mathrm{VC}$ & Vinylchlorid \\
\hline
\end{tabular}




$\begin{array}{llll}\text { min } & \text { Minute } & \text { VDM } & \begin{array}{l}\text { Verband der Deutschen } \\ \text { Möbelindustrie e. V. }\end{array} \\ \text { Mio. } & \text { Million(en) } & \text { vergl. } & \text { vergleiche } \\ \mathrm{ml} & \text { Milliliter } & \overline{\mathrm{X}} & \text { arithmetischer Mittelwert } \\ \mathrm{mm} & \text { Millimeter } & \text { z. B. } & \text { zum Beispiel } \\ \text { mmol } & \text { Millimol } & \text { z. T. } & \text { zum Teil } \\ \text { MV } 1 & \text { Materialverbund 1 } & \text { zzgl. } & \text { zuzüglich }\end{array}$




\section{Abstract}

The growing demand for durable, sustainable, regionally and "fairly" produced products has been rising steadily since the early 2000s, especially products made of wood are becoming more and more popular.

Against this background, together with the company Josef Wochner GmbH \& Co KG, Rosenfeld Heiligenzimmern, the idea was born to develop a material based on real wood veneers of alder (Alnus glutinosa L) and oak (Quercus petraea (matt) Liebl. respectively Quercus robur L. ), bonded with a flexible middle layer, which can be used in the packaging environment.

In order to ensure the highest possible dimensional stability, a 5-layer structure was aimed at, each consisting of two cross-glued veneer sheets on a flexible middle layer. This composite material named „Faltfurnier" and its basic materials were investigated and evaluated with regard to their mechanical-technological and chemical properties.

A material combination of veneers and a PVC foil in the middle layer was selected. A PVAc-based adhesive and a thermoplastic adhesive film on PU basis were used for bonding the different layers.

In a first step, the tensile strength of the PVC foil used was investigated under different starting conditions. Here it was shown that the direction of production has a significant influence on the tensile strength. Thus, parallel to the direction of production significantly higher strengths than perpendicular to the direction of production were found. An influence of the process temperature could not be proven.

Within different test series the surface soundness was used as a criterion to define an optimal pressing time, to evaluate the quality of the bonding by means of thermoplastic adhesive film of the veneers on PVC film as well as the bonding of the cross grain and face veneers by means of the adhesive systems PVAc and adhesive film. The tests resulted in an optimal pressing time of $120 \mathrm{~s}$ and incomparison significantly lower surface soundness in the test series with oak than with alder on PVC foil. Furthermore it could be shown that the bonding of the cross grain and face veneers can be replaced by a thermoplastic adhesive film, but leads to lower strengths. A positive aspect here is that the more economic production using the one-shot process would thus be possible.

Due to the differences in the strength of the bond between alder and oak, the veneers were chemically characterised with regard to their $\mathrm{pH}$ values, buffer capacities and extractives, and reasons for the lower strength of oak on PVC film were determined. An influence of the surface structure of the veneers on the surface soundness could not be proven with certainty. 
Finally, a 5-layer overall composite of alder veneer on PVC foil was produced and examined with regard to its bending strength and bending modulus of elasticity, durability of the folding groove as well as its behaviour in strong alternating climate. The tests showed that the bending strength and bending modulus of elasticity were significantly higher when tested perpendicular to the grain direction of the face veneer than when tested parallel to the grain direction.

Cracks and signs of fatigue in the folding groove only became apparent on average after 4,436 repetitions of the folding process using a door testing machine, and the material remained dimensionally stable except for slight warping on its head sides when stored in a highly variable climate. 


\section{Einleitung}

\subsection{Einführung}

Seit Anfang der 2000er Jahre erlebt die Gesellschaft einen „Bio-Boom“. Nachhaltig produzierte Waren werden verstärkt nachgefragt und ein ökologischer Lebensstil zum Wohlfühlfaktor. Dieser Trend konnte sich sogar trotz der Finanzkrise im Jahr 2009 weiter behaupten. Moralisch korrekter Konsum gilt seit spätestens dieser Zeit als schick. Lediglich der „ethische“ Fokus änderte sich in der vergangenen Zeit von Bio und fair hin zu Regionalität auf Produkt- und sozialer Verantwortung auf Produktionsebene (Otto GmbH \& Co KG 2013).

Auch Produkte aus Holz erfahren zunehmender Beliebtheit, denn Holz passt nicht nur aufgrund seiner $\mathrm{CO}_{2}$-Neutralität in diesen Trend, sondern ist regional verfügbar. Zudem stärkt eine erhöhte Holznutzung den Cluster Forst und Holz und sichert somit zahlreiche Arbeitsplätze, denn nach Seintsch 2013 sind in diesem mehr als 1,1 Mio. Menschen beschäftigt. Wie diverse Clusterstudien auf Landes- wie auch Bundesebene zeigen, kommt im Besonderen dem ländlichen Raum eine hohe Bedeutung in Bezug auf Arbeitsplätze zu (vergl. hierzu Steffens und Bombosch 2007).

Vor diesem Hintergrund entstand zusammen mit einem Projektpartner, der Josef Wochner GmbH \& Co.KG aus Rosenfeld-Heiligenzimmern, die Idee einen innovativen Werkstoff auf der Basis von Echtholzfurnieren zu entwickeln, der sowohl optisch als auch haptisch höchsten Ansprüchen genügt und im Verpackungsumfeld eingesetzt werden soll.

Daraus entstand das Konzept Furniere mit einer flexiblen Mittelschicht zu verbinden, die es nach beidseitig symmetrischen Einfräsen zulässt, den Werkstoff in zwei Dimensionen zu falten, um ein 3-dimensionales Objekt zu erhalten. Der Vorteil eines solchen Werkstoffes wäre neben den ästhetischen und ökologischen Aspekten, die Echtholzfurniere mit sich bringen, dass das Endprodukt flach gelagert und auf Paletten ausgeliefert werden könnte und erst beim Einzelhändler oder Endkunden über speziell entwickelte Verschlussmöglichkeiten oder Falttechniken zusammengebaut wird. Ähnlich einem Umzugskarton. Mögliche Anregungen zu solchen Designs kommen aus dem Verpackungsumfeld (vergl. Jackson 2012). Die Designentwicklung entfällt bei dieser Werkstoffentwicklung auf den Projektpartner und ist nicht Teil dieser Dissertation. 
Eine Ausweitung der Verwendung auf Life-Style-Produkte wie Brillen- und Kreditkartenetuis, Aktenmappen u. Ä. war ebenso angedacht. Der Werkstoff soll vor allem im Hochpreissegment Einsatz finden. Dies fordert enorm hohe Ansprüche an die Verarbeitungsqualität und Langlebigkeit. Langlebige Produkte, im Gegensatz zu Wegwerfprodukten, fügen sich zudem besonders gut in den bereits erwähnten moralisch korrekten Konsumtrend ein.

\subsection{Zielsetzung}

In Zusammenarbeit mit dem Projektpartner wurden Anforderungen an den Werkstoff definiert, die im Rahmen der vorliegenden Dissertation umgesetzt werden sollten.

Als wesentliche Werkstoffmerkmale wurden Dauerhaftigkeit, hohe Qualität/Festigkeit der Verklebung und gute Festigkeitseigenschaften festgelegt. Als technologische Zielgrößen wurden hierzu folgende Werte definiert:

- Dauerhaftigkeit: der Werkstoff soll wenigstens 1.000 Faltintervalle unbeschadet überstehen.

- Qualität der Verklebung: Die Abhebefestigkeit des Furniers von der elastischen Mittelschicht soll den Mindestanforderungen an Spanplatten nach DIN EN 312 in Höhe von $0,80 \mathrm{~N}^{*} \mathrm{~mm}^{-2}$ entsprechen.

- Festigkeit: Eine Biegefestigkeit von mindestens $20 \mathrm{~N}^{*} \mathrm{~mm}^{-2}$ soll erreicht werden.

Für den Herstellungsprozess wurden nachstehende Kriterien als Zielstellung definiert:

- Die zu verwendenden Materialien sollen einfach zu handhaben sein.

- Die Produktionszeit des Halbzeugs soll bei gleichzeitig bestmöglichen technologischen Eigenschaften kurz gehalten werden.

- Die Weiterverarbeitung des Halbzeugs soll schnellstmöglich nach dessen Herstellung realisierbar sein.

Weiterhin war es Wunsch des Projektpartners, dass mit dem Halbzeug ein sehr hoher Individualisierungsgrad des Endproduktes erreicht werden kann.

Grundsätzlich wurde beschlossen, dass der Entwicklungsfokus primär auf der technologischen Seite und erst sekundär auf möglichen ökologischen Aspekten liegt. 


\section{Theoretische Grundlagen}

\subsection{Werkstoffe und ihre Klassifizierung}

Als Verbundwerkstoffe respektive Werkstoffverbünde werden Materialien definiert, die aus zwei oder mehr Komponenten (Phasen) bestehen, die nicht ineinander löslich sind und optimierte Eigenschaften für ihre spezifischen Anwendungen aufweisen (Eyerer 2005). Bei dem in der vorliegenden Arbeit entwickelten und untersuchten Werkstoff handelt es sich um einen sogenannten Schichtverbundwerkstoff.

Der Sinn von Verbundwerkstoffen, zu denen auch einige Holzwerkstoffe zählen, ist die Erzeugung eines Werkstoffes nach Maß, mit der Einflussnahme und Variation der mechanischen Eigenschaften, der Homogenität, der Isotropie u. a. (Niemz 2002b).

\subsection{Holz}

Holz ist ein heterogenes, organisches Material, das sich im Wesentlichen aus den vier chemischen Elementen Kohlenstoff (50\%), Sauerstoff (43\%), Wasserstoff (6\%) sowie einem geringen Anteil an Stickstoff $(<1 \%)$, der primär aus Eiweißstoffen stammt, zusammensetzt (Fengel und Wegener 2003).

Die Zellwand der Hölzer besteht aus drei Biopolymeren, deren Anteile je nach Baumart und Wachstumsregion schwanken. Die folgenden Prozentangaben beziehen sich auf Durchschnittswerte von extrakt- und aschefreien sowie trockenen Nutzhölzern:

Cellulose ca. $43 \%$ bis $46 \%$, Hemicellulosen $27 \%$ bis $37 \%$ und Lignine $20 \%$ bis $27 \%$. Etwa $0,5 \%$ bis $10 \%$ entfallen auf Extraktstoffe, die sich in den Hohlräumen der extrazellulären Matrix sowie in den Zelllumina befinden. Der Gewichtsanteil kann zwischen extraktstoffärmeren und extraktstoffreicheren Baumarten stark differieren (Faix 2012).

Auch innerhalb eines Baumes kann der Anteil an Polymeren und Extraktstoffen sowohl zwischen Splint- und Kernholz als auch zwischen Früh- und Spätholz variieren. So ist beispielsweise der Anteil von Lignin und Cellulose im Splint von 
Nadelhölzern höher als in deren Kernholz. Für die Gruppe der Extraktstoffe kehrt sich das Verhältnis um (Fengel und Wegener 2003).

\subsubsection{Holzextraktstoffe}

Als Extraktstoffe werden organische und anorganische Verbindungen bezeichnet, die in den Zelllumina oder innerhalb der Hohlräume der extrazellulären Matrix eingelagert sind. Sie werden auch als akzessorische Bestandteile, Inhalts- oder Begleitstoffe bezeichnet (Faix 2012).

Die Kategorie und die Quantität von Extraktstoffen divergieren nicht nur intraspezifisch, sondern auch innerhalb eines einzelnen Baumes zum Teil erheblich. Eine besonders große Differenz lässt sich beispielsweise zwischen Splint- und Kernholz feststellen.

Auch interspezifisch variiert der Gehalt an Extraktstoffen teils eminent. So genannte extraktstoffarme Arten wie etwa die Fichte (Picea abies L.) weisen einen Extraktstoffgehalt von ca. $2 \%$ bis $5 \%$ auf, während hingegen extraktstoffreiche Hölzer über einen Anteil von bis zu $25 \%$, manche Tropenhölzer gar bis zu $40 \%$ verfügen (Bodig und Jayne 1982; Roffael und Schäfer 2002).

Grundsätzlich wird zwischen primären und sekundären Extraktstoffen unterschieden, wobei die Sekundären als weitaus bedeutender eingestuft werden (Roffael und Schäfer 2002).

$\mathrm{Zu}$ ihnen zählen Stoffklassen wie Harze, Terpene, Farbstoffe u v.m. (Fengel und Wegener 2003)

\subsubsection{Die Bedeutung von Holzextraktstoffen in technologischer Hinsicht}

Extraktstoffe haben zum Teil erhebliche Auswirkungen auf technologische Prozesse. Während sie keine bzw. keine direkten Auswirkungen auf die mechanischen Eigenschaften von Holz zeigen, können sie jedoch physikalische Eigenschaften wie Hydrophobierung, Dimensionsstabilität $u$. ä. beeinflussen.

Weiterhin sind positive Eigenschaften wie Schutz vor Fäule-, Pilz- oder Insektenbefall auf Extraktstoffe zurückzuführen (Bodig und Jayne 1982).

Insbesondere im Hinblick auf die Verklebung von Hölzern können Extraktstoffe und der pH-Wert des Holzes einen negativen Einfluss bei der Verarbeitung ausüben. So können diese für eine Verschlechterung der Benetzbarkeit mit Bindemitteln verantwortlich sein (Roffael und Rauch 1974; Jaic et al. 1996)

Darüber hinaus wirken sich Extraktstoffe beispielsweise negativ auf das Abbinden von Zement aus. In diesem Fall sind die beteiligten Stoffe die Zuckeranteile und auch phenolische Verbindungen wie Tanin und Conidendrin (Roffael und Rauch 1974; Sandermann und Brendel 1956). 
Weiterhin können bei der Verarbeitung von diversen Holzarten die darin enthaltenen Extraktstoffe gesundheitsgefährden sein (Dadswell und Hillis 1962).

\subsection{Furniere}

Als Furniere werden dünne Blätter bezeichnet, die durch Schälen, Messern oder Sägen vom Stamm oder Stammteil abgetrennt werden. Sie finden als Deck-, Außen-, Innen-, Unter- oder Absperrfurniere Verwendung (Nationale Norm DIN 68330).

Die Herstellung und Nutzung von Furnieren bzw. deren Vorläufer geht weit in der Menschheitsgeschichte zurück. Archäologen datieren ägyptische Funde von Sperrholz auf ca. 2000 Jahre vor Christus (Soiné 1995).

Als reine Zierde wurden beispielsweise Furniere aus Ebenholz auf einer Truhe, die im Grabe Tutenchamuns gefunden wurde, verwendet. Neben dem Gebrauch als Schmuck war aber auch schon der technische Vorteil von Furnieren bekannt. So werfen sich Blindhölzer, auf denen Furniere aufgebracht wurden, weit weniger als massive Bretter.

Ein weiterer Beweggrund für die Nutzung von Furnieren ist der sparsame Einsatz edler und seltener Hölzer. Auf diese Weise lassen sich erheblich größere Oberflächen aus dem gleichen Stück Holz herstellen als in Massivbauweise (Kollmann 1962).

\subsubsection{Grundlegendes zu Furnieren}

Primäre Voraussetzung für die Herstellung von Furnieren ist es, die zu verarbeitenden Stämme vor Austrocknung zu schützen. Dies kann beispielsweise durch Beregnung oder Nasslagerung geschehen (Soiné 1995).

In einem nächsten Schritt werden die zu verarbeitenden Furnierstämme gedämpft oder gekocht, um innere Spannungen des Stammes abzubauen und die elastomechanischen Eigenschaften zu verändern. Hierdurch soll die notwendige Plastifizierung vor dem Messern oder Schälen erreicht werden. Gleichzeitig kann das Dämpfen von Holz zu höheren Standzeiten der Werkzeuge, einer erleichterten Entrindung sowie zum Abtöten von Pathogenen führen (Lohmann 2003; Lohmann 2010).

Im Gegensatz zu unerwünschten Holzverfärbungen durch fehlerhafte Lagerung werden Verfärbungen durch gezielte Behandlungsmethoden bewusst provoziert. So erhält beispielsweise das Holz der Rotbuche (Fagus sylvatica L.) durch eben diese exakt gesteuerte Dämpf- oder Kochprozesse eine rötliche Färbung (Koch et al. 2000).

Die Prozessparameter (Temperatur, Dauer etc.) beim Dämpfen oder Kochen variieren je nach Holzart z. T. erheblich und gelten als Firmengeheimnisse. 


\subsubsection{Herstellungsverfahren}

Furniere werden hinsichtlich ihrer Herstellungsverfahren in folgende Kategorien unterteilt:

1. Sägefurniere

Die älteste Form der Furniere ist das Sägefurnier. Es wird mittels Furniergattern oder -sägen hergestellt. Aufgrund des hohen Verschnittanteiles ist es heute eher selten geworden. Sägefurniere zeigen meist einen Fladern- oder Radialschnitt (Bablick 2009).

Um die Schnittfugenverluste möglichst gering zu halten, werden Sägefurniere meist erst ab einer Materialstärke von $1,5 \mathrm{~mm}$ produziert. Sie werden meist für Anwendungen im Außenbereich oder in starkem Wechselklima eingesetzt (Lohmann 2003).

2. Schälfurniere

Bei der Herstellung von Schälfurnieren wird zwischen zwei Verfahren unterschieden:

- Rundschälen: Beim Rundschälen werden die gedämpften, noch warmen Stammblöcke zentriert auf die Spindel (Pinole) der Schälmaschine gespannt. Das Schälmesser wird bei diesem Verfahren gegen den rotierenden Stammblock gefahren (Soiné 1995).

- Exzenterschälen: Das Stammstück wird exzentrisch eingespannt, um sich ändernde Schnittebenen zu erhalten. Durch diese Art der Einspannung werden die Jahrringe zunächst tangential und dann radial angeschnitten, was dem Bild eines Messerfurnieres entspricht.

Als Sonderform bzw. Weiterentwicklung des Exzenterschälens kann das Stay-logVerfahren angesehen werden. Bei diesem Verfahren wird der Stamm extrem exzentrisch eingespannt, was einen deutlich flacheren Schnitt durch die Jahrringe zur Folge hat (Bablick 2009).

3. Messerfurniere

Als Messerfurniere bezeichnet man solche, die von einem aufgespannten Stamm oder Stammteil durch ein parallel zur Auflageebene waagerecht oder senkrecht wirkendes Messer abgetrennt werden (Nationale Norm DIN 68330).

Beim Messern wird vor allem zwischen zwei Schnittarten unterschieden:

-Quartierschnitt: Bei dieser Schnittart wird der Stamm in s. g. Flitches geviertelt (Quartier $=$ Viertel). Dabei werden die Markstrahlen mehr oder weniger längs angeschnitten, woraus sich glänzende, horizontale Bänder ergeben, die als Spiegel bezeichnet werden (Nationale Norm DIN 68330). 
- Fladerschnitt bzw. Fauxquartierschnitt: Hier werden die Flitches sehnenartig zu den jeweils berührten Jahrringen tangential (in Faserrichtung) geschnitten. Bei diesem Schnitt kommt die natürliche Zeichnung des Holzes besonders gut zur Geltung, da die Markstrahlen senkrecht bis schräg durchtrennt werden (Nationale Norm DIN 68330).

Eine besondere Form des Messerfurniers stellt das so genannte Mikrofurnier dar. Es ist zwischen $0,1 \mathrm{~mm}$ bis 0,15 $\mathrm{mm}$ stark und wird u. a. zu Echtholztapeten verarbeitet (Bablick 2009). 


\subsubsection{Furniere und ihre wirtschaftliche Bedeutung}

War der deutsche Furniermarkt Mitte der 1990er Jahre noch der größte in Europa, zeigen aktuelle Zahlen einen gegenläufigen Trend. Innerhalb von zwei Jahrzehnten reduzierte sich das Marktvolumen von ehemals 300 Mio. Euro im Jahre 1995 auf rund 65 Mio. Euro im Jahr 2016 (siehe Abbildung 2-1) (IHB 2014; VDM 2017).

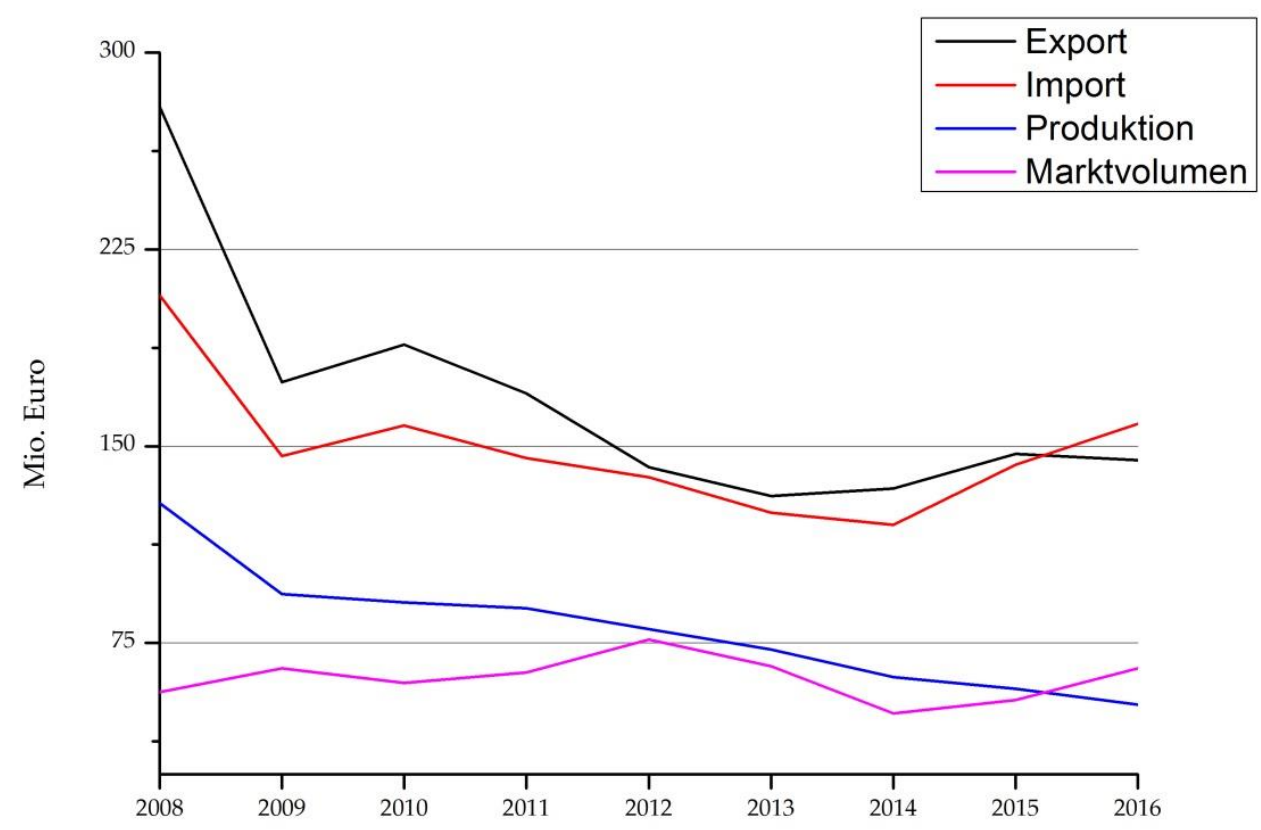

Abbildung 2-1: Marktentwicklung Furnier in Deutschland 2008-2016 in Mio. Euro nach (VDM 2017).

Als zentrale Ursache für die schlechte Marktsituation der Furnierbranche gelten Furniersubstitute wie Dekorfolien, lackierte Oberflächen, Imitate und ähnliche. Diese werden echtem Holz sowohl haptisch als auch optisch immer ähnlicher und sind selbst für Experten nur sehr schwer als Imitation zu identifizieren.

Ein weiterer Nachteil von Furnieren gegenüber Substituten sind die deutlich höheren Kosten, was v. a. Möbelhersteller, entgegen der Kundenwünsche, auf kostengünstigere Dekore umsteigen lässt (Schrott 2010; Behets-Oschmann und Knauf 2010).

Doch trotz des langfristigen Abwärtstrends der Furnierbranche sieht eben diese eine mögliche Trendwende. Nach vielen Jahren der negativen Absatzentwicklung ist, nach Aussage einiger Furniererzeuger, erstmals wieder ein leichter Umsatzanstieg zu registrieren. Eine Sonderstellung unter den Furniererzeugern haben die Zulieferer der Holzfußbodenhersteller inne. Diese erwarten im Gegensatz zum Rest der Branche ein stärkeres Wachstum (IHB 2015).

Die dargestellte Situation des Furniermarktes zeigt den drängenden Bedarf an Innovationen. Die etablierten Player haben auf diese Nachfrage reagiert und neben den 
klassischen Furnieren neue Entwicklungen angeschoben. So drängen derzeit neben Räucher-, sägerauen-, flexiblen- und 3D-Furnieren auch Thermofurniere und weitere Entwicklungen auf den Markt.

\subsection{Polyvinylchlorid}

Polyvinylchlorid (PVC) ist ein thermoplastischer Kunststoff, der mit 27 Mio. t/a an dritter Stelle des Verbrauchs sämtlicher Kunststoffe steht. Seine Entwicklungsgeschichte geht bis auf das Jahr 1835 zurück. Henri Victor Regnault gewann den Ausgangsstoff für die PVC-Produktion, Vinylchlorid, indem er Chlor an Ethylen anlagerte.

PVC wird nach den drei Polymerisationsverfahren Emulsionspolymerisation, Suspensionspolymerisation und der Massepolymerisation hergestellt, wobei die Rohstoffe zur Produktion zu $43 \%$ aus Erdöl und zu $57 \%$ aus Steinsalz gewonnen werden (Diemert et al. 2005).

\subsubsection{Herstellungsverfahren von PVC}

Im Folgenden soll kurz auf die drei verschiedenen Polymerisationsverfahren eingegangen werden (Tabelle 2-1 gibt einen Überblick über die verschiedenen Eigenschaften von Emulsions-, Suspensions-, und Massepolymerisations-PVC):

1. Emulsionspolymerisation (E-PVC)

Ein System aus Wasser und Vinylchlorid wird durch Emulgatorenzusatz und unter Rühren in eine stabile Emulsion überführt. Nach der durch wasserlösliche peroxidische Initiatoren aktivierten Polymerisation wird das Polymerisat durch Sprühtrocknen $\mathrm{zu}$ feinen, verpastbaren oder $\mathrm{zu}$ groben, rieselfähigen Typen verarbeitet. Die Teilchengröße divergiert dabei (typenabhängig) zwischen ca. $10 \mu \mathrm{m}$ und $300 \mu \mathrm{m}$ (Diemert et al. 2005).

2. Suspensionspolymerisation (S-PVC)

Diemert et al. 2005 beschreiben den Prozess der Suspensionspolymerisation wie folgt:

"Vinylchlorid wird durch intensives Rühren im Wasser eines Autoklaven in feinste Tröpfchen zerteilt. Schutzkolloide verhindern das Zusammenfließen der Tröpfchen. Vinyllösliche Initiatoren lösen die Polymerisation aus. Aus den Monomer-Tröpfchen werden feste Polymerteilchen (60 $\mu \mathrm{m}$ bis $250 \mu \mathrm{m}$ Durchmesser), die durch Zentrifugieren von der wässrigen Phase getrennt werden. Danach wird das Produkt gewaschen und getrocknet. 
Bei diesem Verfahren werden salz- und emulgatorfreie Polymere gewonnen; sie enthalten jedoch noch geringe Mengen an Schutzkolloid. Die elektrischen Werte sind gut, daraus hergestellte Folien und Flaschen glasklar."

3. Massepolymerisation (M-PVC)

Bei der Massepolymerisation handelt es sich um ein zweistufiges Verfahren, bei dem monomerlösliche Peroxide als Initiatoren Verwendung finden. Diemert et al. 2005 beschreiben dieses Zweistufenverfahren folgendermaßen:

"Im Vor-Polymerisator wird in einer ersten Stufe ein VC-Umsatz von $5 \%$ bis $10 \%$ bei sehr hoher Rührgeschwindigkeit erreicht. Diese Suspension wird in einem zweiten HauptPolymerisator mit weiterem VC und Initiatorzusatz bis zu einem Endumsatz von $60 \%-$ $80 \%$ polymerisiert. Das monomerfeuchte Polymer wird ausgegast, gesiebt und den Silos zugeführt.M-PVC ist sehr rein, die Korngröße ca. 100 um und die Kornverteilung eng."

Tabelle 2-1: Kennzeichnende Eigenschaften von E-, S-, und M-PVC in Anlehnung an (Kaiser 2007).

\begin{tabular}{|l|l|l|l|}
\hline $\begin{array}{l}\text { PVC- } \\
\text { Typ }\end{array}$ & $\begin{array}{l}\text { Korngröße vor } \\
\text { der Weiter- } \\
\text { verarbeitung }\end{array}$ & $\begin{array}{l}\text { Verunreinigungen } \\
\text { (Gew.\% } \\
\text { Sulfatasche) }\end{array}$ & \multicolumn{1}{|c|}{ Bemerkungen } \\
\hline E-PVC & $\begin{array}{l}0,10 \mu \mathrm{m}- \\
2,00 \mu \mathrm{m}\end{array}$ & $\begin{array}{l}\text { hoch (bis über 1,60 } \\
\%)\end{array}$ & $\begin{array}{l}\text { sehr gut gelierbar, mit } \\
\text { Weichmacher verpastbar; für } \\
\text { Dryblendverarbeitung bedingt } \\
\text { geeignet }\end{array}$ \\
\hline S-PVC & $\begin{array}{l}0,02 \mathrm{~mm}- \\
0,20 \mathrm{~mm}\end{array}$ & mittel (unter 0,10\%) & $\begin{array}{l}\text { wichtigster PVC-Typ; für Hart- } \\
\text { und Weich-PVC-Verarbeitung } \\
\text { mit guten mechanischen, } \\
\text { optischen und elektrischen } \\
\text { Eigenschaften }\end{array}$ \\
\hline M-PVC & $0,06 \mathrm{~mm}-$ & gering (unter 0,01 \%) & $\begin{array}{l}\text { hochreiner PVC-Typ mit besten } \\
\text { mechanischen, optischen und } \\
\text { elektrischen Eigenschaften }\end{array}$ \\
\hline
\end{tabular}

PVC wird in zwei Ausprägungsformen unterschieden. Zum einen in der harten Ausprägungsform PVC-U (Weichmacherfreies PVC) und zum anderen in der weichen Form, dem PVC-P (hoher Weichmacheranteil) (Knippers 2010). 


\subsubsection{PVC und seine wirtschaftliche Bedeutung}

Im Jahr 2013 wurde in Deutschland 1,84 Mio.t PVC produziert. Diese Produktionsmenge entsprach dabei einem Anteil von $17,5 \%$ an der deutschen Gesamtkunststoffproduktion. Somit war PVC der am zweit häufigsten erzeugte Kunststoff in Deutschland. Mit einem Anteil von 17,9\% an der Gesamtproduktion wurde nur Polypropylen (PP) häufiger fabriziert (consultic 2014a).

Mit einer Produktionsmenge von $440 \mathrm{kt}$ stellen Fensterprofile den wichtigsten Einsatzbereich von PVC dar, gefolgt von Rohren mit $230 \mathrm{kt}$ und Verpackungen mit $194 \mathrm{kt}$.

Abbildung 2-2 gibt eine Übersicht über weitere Produktgruppen und deren Produktionsmenge.

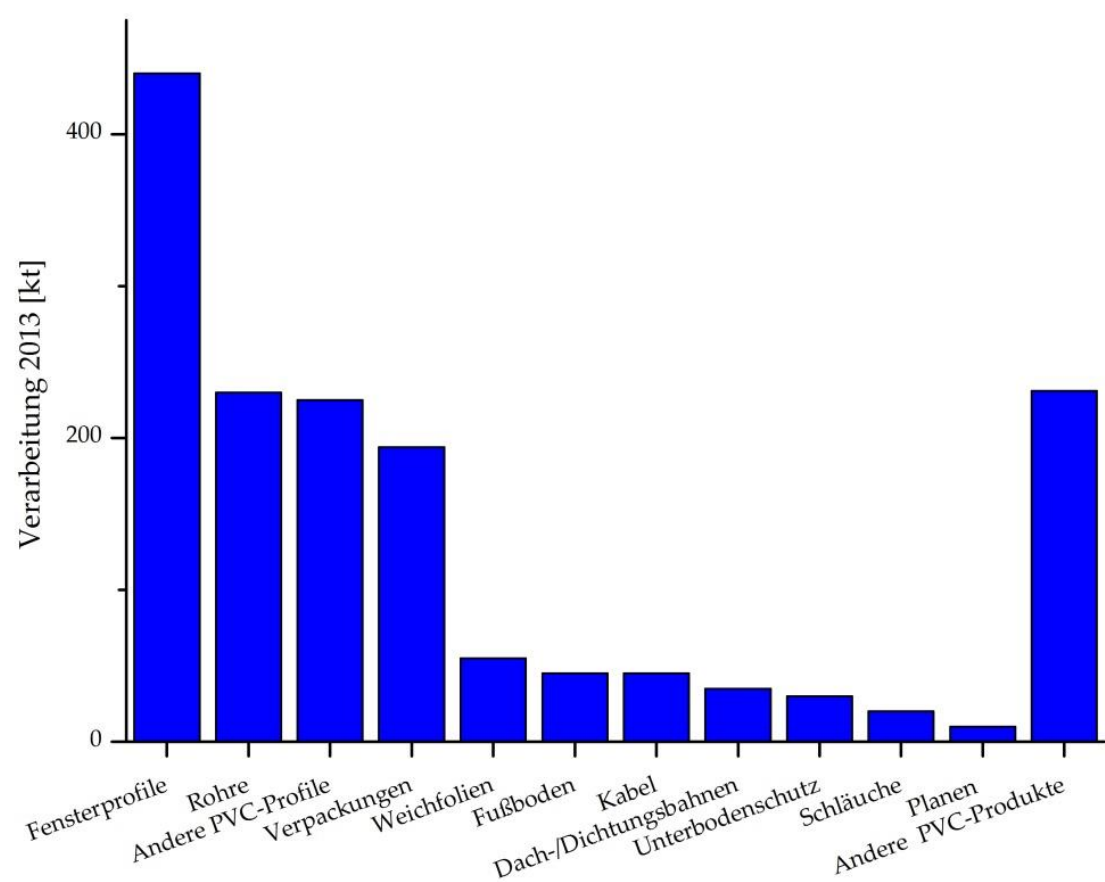

Abbildung 2-2: PVC Verarbeitung nach Produktgruppen im Jahr 2013 (Daten nach (consultic 2014b)). 


\subsection{Klebstoffe}

Als Klebstoff wird nach DIN EN 923 ein „,nichtmetallischer Stoff, der Werkstoffe durch Oberflächenhaftung (Adhäsion) so verbinden kann, dass die Verbindung eine ausreichende innere Festigkeit (Kohäsion) besitzt", bezeichnet.

Klebstoffe werden zum einen nach ihrer chemischen Basis (organisch oder anorganisch $^{1}$ ) und zum anderen nach ihrem Abbindemechanismus (siehe Abbildung 2-3) eingeteilt (Habenicht 2009).

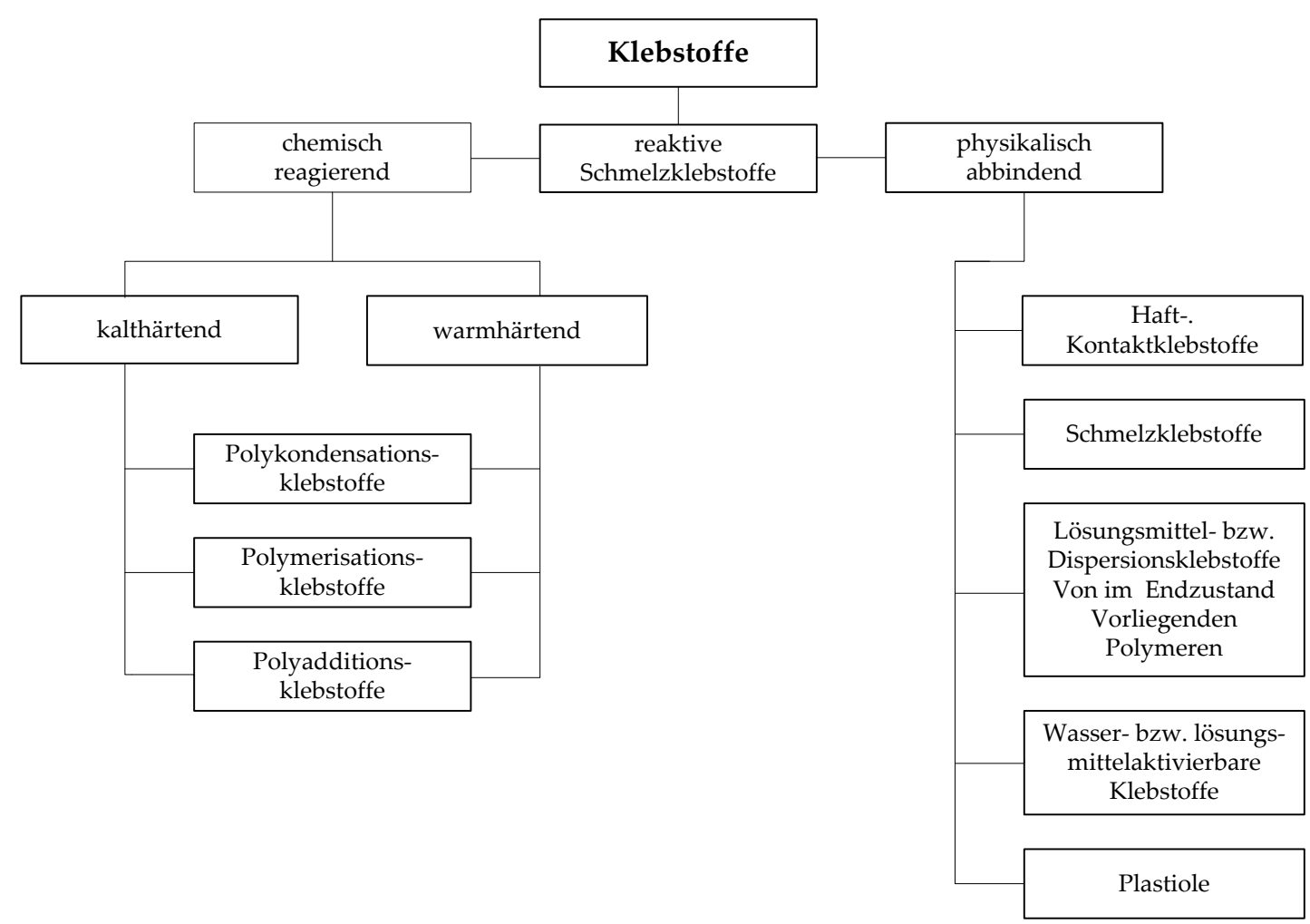

Abbildung 2-3: Klebstoffeinteilung nach dem Abbindemechanismus (Habenicht 2009).

\subsubsection{Aufbau von Klebstoffen}

Die wesentlichen Klebstoffbestandteile, also diese, die man als Grundstoff ansieht, sind die Monomere, Prepolymere oder Polymere. Sie bilden das Grundgerüst der makromolekularen Struktur und sind maßgeblich an der Ausbildung der Klebschicht beteiligt (Habenicht 2009).

Polymere unterscheiden sich hinsichtlich ihrer Struktur, was zu divergierenden chemischen, physikalischen und mechanischen Eigenschaften führt.

\footnotetext{
${ }^{1}$ Silicone nehmen eine Sonderstellung ein, da sie organische wie auch anorganische Merkmale aufweisen (Habenicht 2009).
} 
Von besonderer Bedeutung ist hierbei das Verhalten unter Temperaturbeanspruchung (Habenicht 2009).

Generell lassen sich Polymere bezüglich ihrer Eigenschaften, die auf ihrer Molekülstruktur basieren, in folgende Arten unterteilen (Habenicht 2009):

- Thermoplaste

- Duromere

- Elastomere

Einen besonderen Stellenwert nehmen in dieser Arbeit die Thermoplaste und Elastomere ein. Der Vollständigkeit halber sollen in den folgenden Kapiteln jedoch alle drei Polymerarten kurz charakterisiert werden.

\subsubsection{Thermoplaste}

Bei Thermoplasten handelt es sich um linear oder verzweigt aufgebaute Makromoleküle, die abhängig von ihrem Kettenaufbau in amorphem oder teilkristallinem Zustand vorliegen können. Thermoplaste erweichen bei Erwärmung bis zur Fließbarkeit und verfestigen sich nach dem Abkühlen wieder (reversible Zustandsänderungen) (Habenicht 2009).

\subsubsection{Duromere}

Bei der Gruppen der Duromere handelt es sich um Makromoleküle, die nach dem Aushärten in einem starren, amorphen und teils spröden Zustand vorliegen. Auch bei hohen Temperaturen lassen sich diese räumlich engvernetzen Makromoleküle plastisch nicht verformen. Dieses Merkmal beruht auf den vorliegenden kovalenten Bindungskräften, die ein gegenseitiges Verschieben in der Polymerstruktur nicht zulassen. Duromere sind in praktisch allen organischen Lösungsmitteln unlöslich (Habenicht 2009).

\subsubsection{Elastomere}

Nach Habenicht (2009) handelt es sich bei Elastomeren um: "weitmaschig vernetzte Makromoleküle, die bis zum Temperaturbereich chemischer Zersetzung nicht fließbar werden, sondern weitgehend temperaturunabhängig gummielastisch reversibel verformbar sind (z. B. Kautschukderivate"). 


\subsection{Bindungskräfte}

Das flächige Verbinden gleicher oder wie im Fall der vorliegenden Arbeit verschiedenartiger Werkstoffe unter Zuhilfenahme einer oft artfremden Substanz, die an den zu verbindenden Fügeteilen haftet und dort Kräfte überträgt, bezeichnet man als Kleben (Brockmann et al. 2005).

\subsubsection{Adhäsion}

Das makroskopische zweidimensionale (flächige) Aneinanderhaften artgleicher oder artfremder Substanzen wird als Adhäsion bezeichnet und gilt als eines der wichtigsten stofflichen Phänomene in Natur und Technik. Die Adhäsionsforschung hat über langjährige Beobachtungen festgestellt, dass adhäsive Verbindungen niemals dort trennbar sind oder versagen, wo tatsächliche Adhäsion entstanden ist (Brockmann et al. 2005).

Nach aktuellen Theorien werden folgende Adhäsionsarten unterschieden:

1. Spezifische Adhäsion

Unter dem Begriff der spezifischen Adhäsion "werden die auf chemischen, physikalischen und thermodynamischen Gesetzmäßigkeiten beruhenden Adhäsionserscheinungen verstanden. Sie stellen die wesentliche Ursache für die Ausbildung der Adhäsionskräfte in Klebungen dar" (Habenicht 2009).

2. Mechanische Adhäsion

Im Falle der mechanischen Adhäsion handelt es sich primär um eine formschlüssige Verankerung der ausgehärteten Klebschicht, die aus einer flüssigen Phase hervorging, in Poren, Kapillaren sowie Hinterschneidungen der Fügeteile. Zusätzlich zur mechanischen Adhäsion können Erscheinungen der spezifischen Adhäsion wirksam werden (Habenicht 2009).

3. Autohäsion

Bei der Fusion kautschukelastischer Polymerschichten des gleichen Materials (nahezu $\mathrm{zu}$ ausschließlich dort) tritt unter Voraussetzung einer hohen Beweglichkeit der Makromoleküle, die unter Druckanwendung zu einer bilateralen Diffusion mit nachfolgender Verklammerung von Kettensegmenten fähig sind, die s. g. Autohäsion auf (Habenicht 2009). 


\subsubsection{Kohäsion}

Die Kohäsion wird häufig auch als „innere Festigkeit“ bezeichnet. Sie beschreibt das Wirken von Anziehungskräften zwischen Atomen respektive Molekülen innerhalb eines Stoffes. Somit wird die Abgrenzung zur Adhäsion deutlich, die das agieren der Anziehungskräfte zwischen differenten Stoffen beschreibt (Habenicht 2009).

Die Kohäsion ist ursächlich für die Oberflächenspannung verantwortlich. Die Art der Bindungskräfte entspricht den Wechselwirkungen, die auch bei Adhäsion auftreten (Kharazipour 1996; Euring 2008). 


\subsection{Faltfurnier}

\subsubsection{Aufbau von Faltfurnier}

Hinsichtlich der verwendeten Materialien bzw. deren Schichtung besteht das Faltfurnier (FF) aus drei Schichten, basierend auf zwei verschiedenen Werkstoffen. Bei genauerer Betrachtung handelt es sich um einen, die Klebefugen ausgenommen, fünfschichtigen Materialaufbau (vergl. Abbildung 3-7) bestehend aus einer elastischen Mittelschicht (in der vorliegenden Arbeit PVC), die beidseitig mit einem Deck- und einem Absperrfurnier (Unterfurnier) verklebt ist.

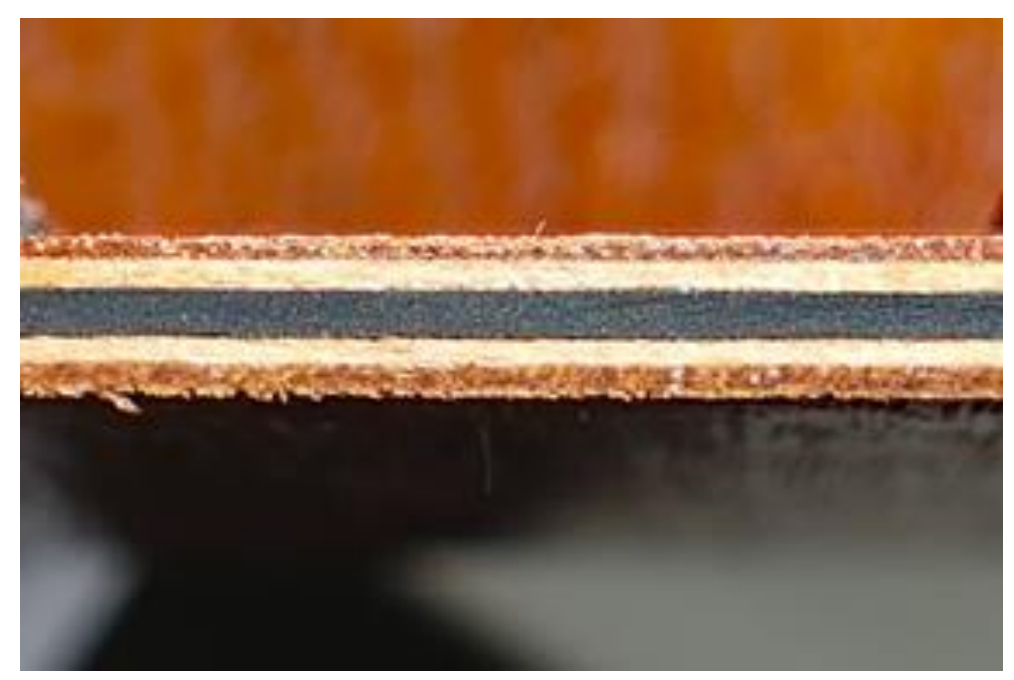

Abbildung 2-4: Nahaufnahme einer Faltfurnierplatte zur Darstellung des 5-schichtigen Aufbaus.

Abbildung 2-4 zeigt durch die Verwendung zweier farblich unterschiedlicher Furniere den 5-schichtigen Aufbau des Werkstoffes. $\mathrm{Zu}$ erkennen sind die elastische Mittelschicht aus PVC-Folie (schwarze Schicht), das helle Absperrfurnier aus Erle und das dunkle Deckfurnier aus Mahagoni (hier verwendet um den Kontrast besser darstellen zu können).

In der vorliegenden Arbeit wurden zwei Klebstoffe, ein Furnierleim und ein thermoplastischer Klebefilm verwendet und untersucht, die der Werkstoffherstellung dienten (vergl. Kapitel 3.1.3).

Als Furniere kamen Erlen- sowie Eichenfurnier zum Einsatz (vergl. Kapitel 3.1.1)

Die eigentliche Funktion, die Faltbarkeit, wird erst durch symmetrisches, beidseitiges Einfräsen der Furnierschicht (siehe Abbildung 2-5) bis auf die Mittelschicht, ohne diese zu beschädigen, ermöglicht. 


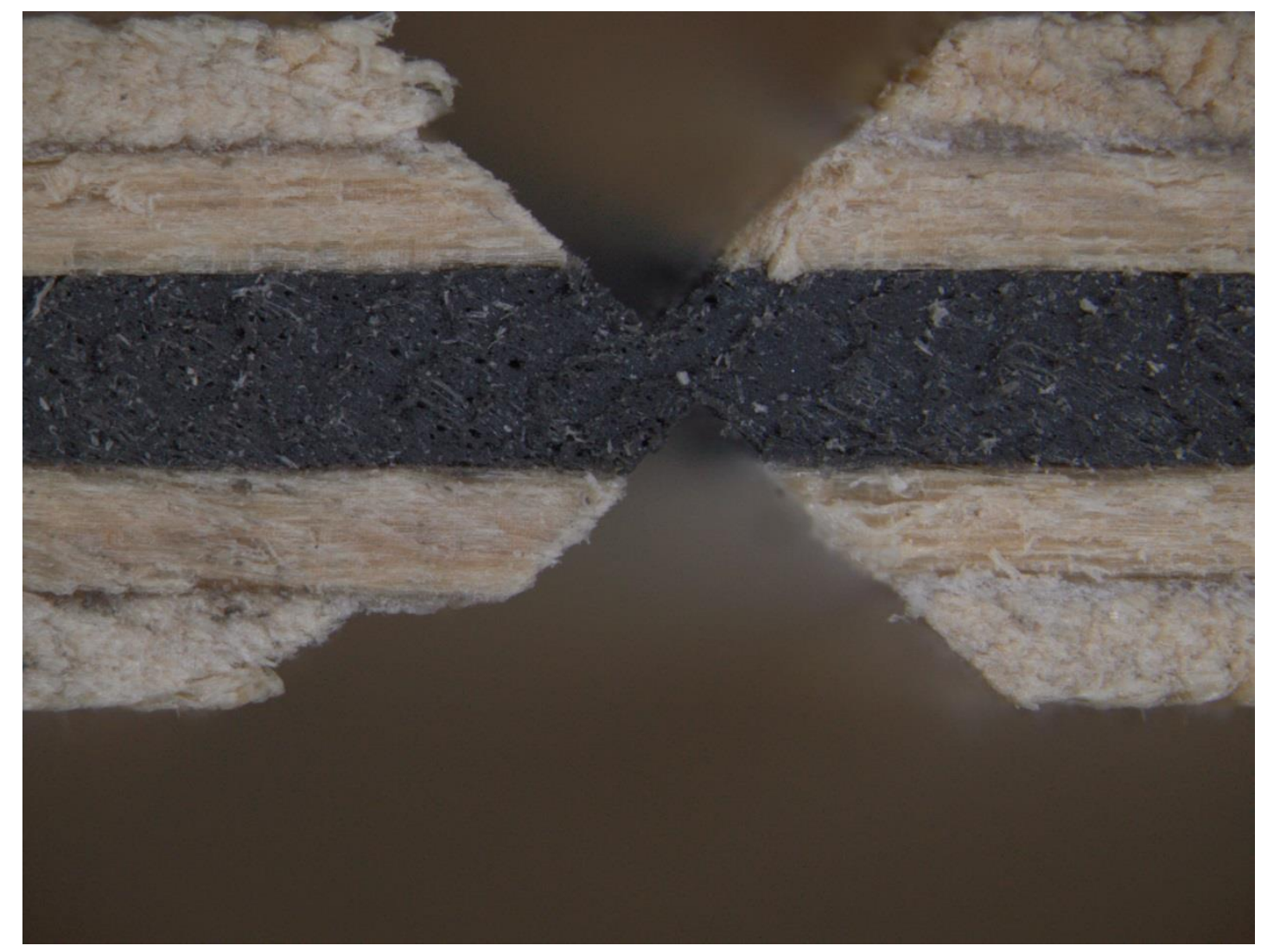

Abbildung 2-5: Seitliche Aufsicht auf eine beidseitig eingefräste Faltfurnierplatte. Hier mit durch den Fräskopf beschädigter Mittelschicht (Foto: P. Werndl).

Die jeweilige Anordnung der Fräsnuten ist abhängig von dem zu produzierenden Produkt. Ein Versatz der Fräsnuten zueinander sowie das Beschädigen der Mittelschicht wie in Abbildung 2-5 zu sehen, ist zu vermeiden. Diese Aspekte werden eingehend in den Kapiteln 4.2.3 und 4.5.2 behandelt. 


\section{Material und Methoden}

\subsection{Material}

In den folgenden Kapiteln werden zum einen die in dieser Arbeit zur Herstellung des Werkstoffverbundes verwendeten Materialen erläutert. Zum anderen werden die technischen und analytischen Geräte beschrieben, die zur Herstellung und zur Untersuchung des Werkstoffverbundes dienten.

\subsubsection{Furniere}

In dieser Arbeit lag der Fokus auf der Verwendung von Furnieren aus zwei Holzarten. Zum einen kamen Messerfurniere aus Erle (Alnus glutinosa L:) zum Einsatz und zum anderen Messerfurniere aus Eiche (Quercus petraea (MATT.) LIEBL. bzw. Quercus robur L.).

Die Entscheidung zur Verwendung dieser beiden Furnierarten erfolgte aus diversen Gründen.

Aus analytischer Sicht decken diese zwei Baumarten zwei Holzartengruppen ab.

Die Eiche gehört zur Art der ringporigen Hölzer, während die Erle den zerstreutporigen Laubhölzern zuzuordnen ist.

Auch chemisch unterscheiden sich diese Hölzer stark. Während das Furnier der Eiche saurer als das der Erle ist, sind auch andere Inhaltsstoffe wie Tannine und Terpene bei der Eiche in wesentlich höheren Konzentrationen vertreten als bei der Erle, was gesteigerte Ansprüche an die Weiterverarbeitung, insbesondere die Verklebung, stellt (vergl. Roffael und Rauch 1974).

Auch die Beschaffenheit der Oberfläche beider Furniere differiert erheblich. Während die Oberfläche bei Erlenfurnier homogen und glatt wirkt, erscheint die des Eichenfurniers sehr rau.

Eine weiterführende Betrachtung der holzanalytischen Thematik erfolgt in Kapitel 4.4.1.

Ebenfalls seien die Kosten genannt. Laut Einkaufspreisen des Projektpartners der Firma Josef Wochner GmbH \&Co. KG, Rosenfeld-Heiligenzimmern sind Messerfurnier aus Erle mit einem Preis von $\sim 6$ Euro $/ \mathrm{m}^{2}$ vergleichsweise günstig und daher als nichtsichtbares Absperrfurnier gut geeignet. Auch die Eiche gehört mit 9 Euro/ $/ \mathrm{m}^{2} \mathrm{zu}$ den kostengünstigeren Furnieren. 


\subsubsection{Material für die Mittelschicht}

Wie in Kapitel 1.1 beschrieben, wurden Zielgrößen definiert, die der Verbundwerkstoff erreichen soll. Diese wurden aus technologischer Sicht um folgende Anforderungen ergänzt. Auf gewebtes Material für die Mittelschicht ist nach Möglichkeit zu verzichten, da das Risiko des Aufspulens eines Fadens auf den Fräskopf und somit eine Materialzerstörung besteht. Weiterhin soll der Werkstoff leichtere Ungenauigkeiten beim Fräsen tolerieren können. Eine Verletzung der Mittelschicht durch den Fräser darf nicht zur Verwerfung des gesamten Halbzeugs führen. Die Verarbeitung des Materials soll keine zu hohen technologischen Ansprüche erfordern.

Da in Vorversuchen einige Materialien wie Kautschukderivate (z. B. Ethylen-PropylenDien-Kautschuk), Filze und zellstoffbasierte Materialien ausgeschlossen wurden, fiel die Wahl auf eine Folie aus Polyvenylchlorid (PVC).

Der Kunstostoff PVC ist ein häufig verwendetes Material, dass in Kapitel 2.4 bereits intensiv beschrieben wurde und dessen Eigenschaften gut erforscht sind.

Die in dieser Arbeit verwendete Folie wurde von der Firma Heissner, Lauterbach bezogen. Das Material hat eine Dicke von $1 \mathrm{~mm} \pm 8 \%$, ein Gewicht von $1.270 \mathrm{~g}^{*} \mathrm{~m}^{-2}$ sowie eine Dichte von „1,36 $\mathrm{g}^{*} \mathrm{~m}^{-2} \pm 0,03 \mathrm{~g}^{*} \mathrm{~m}^{-2}$ [sic! ]“ (Heissner GmbH o. D.).

\subsubsection{Klebstoffe}

Sowohl bei der Herstellung der Faltfurnierplatten als auch bei der Herstellung der Prüfkörper kamen verschiedenste Klebstoff zur Anwendung.

\subsubsection{Furnierleim auf Polyvinylacetatbasis}

Zur Verklebung- bzw. Verleimung der Deckschicht auf der Absperrschicht wurde ein Einkomponentenleim ohne Formaldehydzusatz auf Basis von Polyvinyacetat-(PVAc) (Spezialdispersion) verwendet. Der Handelsname dieses Leims lautet Kleiberit Furnierleim 322. Hersteller ist die Firma Klebchemie M. G. Becker GmbH \& Co. KG, Weingarten/Bd. (Markenname Kleiberit). Die Lieferform erfolgt flüssig und gebrauchsfertig, sodass keine weitere Mischung nötig ist. Laut Herstellerangaben beträgt die Dichte des Leimes ca. $1,2 \mathrm{~g}^{*} \mathrm{~cm}^{-3}$, der $\mathrm{pH}$-Wert liegt bei ca. 5 und die Farbe ist weiß. Die offene Zeit beträgt bei $20^{\circ} \mathrm{C}$ bis zu $6 \mathrm{~min}$. Der empfohlene Leimauftrag liegt nach Herstellerangaben, abhängig von der Oberflächenstruktur, bei $120-180 \mathrm{~g}^{*} \mathrm{~m}^{-2}$ und der empfohlene Pressdruck bei $0,2 \mathrm{~N}^{*} \mathrm{~mm}^{-}$ $2-0,5 \mathrm{~N}^{*} \mathrm{~mm}^{-2}$.

Die Wahl diesen Klebstoff zu verwenden geschah aus mehreren Gründen. Zum einen ist die Handhabung bekannt und relativ einfach (Schutzausrüstung, Absaugung u. ä. sind nicht notwendig), zum anderen kann dieser Klebstoff mit allen hier verwendeten 
Furnierarten problemlos angewendet werden. Eine negative Interaktion zwischen Klebstoff und z. B. Holzinhaltsstoffen ist hier nicht bekannt.

Mögliche negative Auswirkungen des Klebstoffes auf die Oberfläche des Deckfurnieres wie Leimdurschlag, Risse, Durchscheinen von Absperrfurnieren u. ä (vergl. hierzu Müller 1984) sind im Zusammenhang mit den Versuchsreihen irrelevant.

\subsubsection{Klebefilm}

Als Klebstoff zum Fügen der Polyvinylchlorid-Folie mit den verschiedenen Furnierarten wurde grundsätzlich ein thermoplastischer Klebefilm auf Basis von Polyurethanen verwendet, der den Handelsnamen „46.002 (V 807-2)“ trägt. Dieser Klebefilm stammt von der Firma Collano Adhesives (kurz Collano), Sempach-Station, Schweiz. Die Dichte dieses Klebstoffes liegt nach Herstellerangaben bei $1,16 \mathrm{~g}^{*} \mathrm{~cm}^{-3}$, der Schmelzbereich bei $65{ }^{\circ} \mathrm{C}$ bis $85^{\circ} \mathrm{C}$, der Schmelz-Index bei $5 \mathrm{~g} / 10 \mathrm{~min}$ bis $16 \mathrm{~g} / 10 \mathrm{~min}$ und seine Wärmestandfestigkeit liegt, nach interner Methode der Firma Collano, bei $60{ }^{\circ} \mathrm{C}$. Die Lieferform erfolgt als Film auf einem flachen gelben Polyethylen-Träger, der vor dem Verarbeiten entfernt werden muss.

\subsubsection{Cyanacrylatklebstoff}

Für die Prüfung der Abhebefestigkeit ist es nötig, einen normgerechten, metallischen Prüfstempel mit dem Prüfkörper zu verkleben. $\mathrm{Zu}$ diesem Zweck wurde ein Cyanacrylatklebstoff (umgangssprachlich Sekundenkleber) mit dem Handelsnamen 405.08 der Firma Jowat, Detmold verwendet. Der Festkörperanteil liegt nach Herstellerangaben bei ca. $100 \%$ und die Dichte bei $1,05 \mathrm{~g}^{*} \mathrm{~cm}^{-3}$.

\subsubsection{Technische Geräte zur Herstellung von Faltfurnierplatten}

\section{$\underline{\text { Heißpresse }}$}

Zur Herstellung des Halbzeuges wurde eine hydraulische EinetagenUnterkolbenpresse der Firma Bürkle, Freudenstadt (Modell LA 100; Baujahr 1980) verwendet.

Die Pressfläche beträgt $800 \mathrm{~mm} \times 800 \mathrm{~mm}$. Die Kolbenfläche misst $314 \mathrm{~cm}^{2}$. Nach Angaben des Herstellers handelt es sich um eine mittels Heizpatronen elektrisch beheizte Laborpresse. Sie besitzt eine Niederdruck- sowie eine Hochdruckstufe, die jeweils mit eigenem Manometer ausgestattet sind. Der maximale Betriebsdruck der Laborpresse liegt bei 31,39 MPa (max. Flächenpressung 11,28 MPa). Der obere und untere Presstisch kann jeweils bis maximal $250{ }^{\circ} \mathrm{C}$ aufgeheizt werden.

Das Verpressen des Materialverbundes erfolgt zwischen zwei Pressplatten aus Aluminium. 


\section{Furnierpresse}

Die Kaltverleimung der Deckschicht erfolgt auf einer hydraulischen EinetagenFurnierpresse der Firma Ott, Waiblingen-Neustadt (Modell Junior 60). Die Presstische werden elektrisch beheizt und haben eine Fläche von $2200 \mathrm{~mm}$ x 1100 mm. Der untere Presstisch wird durch vier Hydraulikzylinder bewegt. Die Kolbenfläche beträgt $4 \times 50,2 \mathrm{~cm}^{2}$ (Summe: 200,8 $\mathrm{cm}^{2}$ ). Die maximale Presstemperatur liegt nach Thermostatanzeige bei $150{ }^{\circ} \mathrm{C}$.

Formatkreissäge

Zum Besäumen und Zuschneiden der Faltfurnier-Platten wurde eine Formatkreissäge, Typ F 45 (CE-Ausführung) der Firma Altendorf, Minden verwendet. Die Formatkreissäge ist mit einem doppelseitigen Gehrungsanschlag ausgestattet, welcher über eine CNC-gesteuerte Verstellung verfügt, die Maßeinstellungen von bis zu 1/10 mm Genauigkeit zulässt.

\section{Bearbeitungszentrum}

Die Probekörperherstellung für Zugversuche des Halbzeuges wurden auf einem 5Achs-Bearbeitungszentrum der Firma HOMAG Group AG, Schopfloch im Hause der Firma Josef Wochner hergestellt.

Breitbandschleifmaschine (Kalibrierer)

Die zur Verklebung der Prüfkörper für die Bestimmung der Abhebefestigkeit nach DIN EN 311 benötigten Multiplex-Platten wurden vor der Verleimung mit einer Breitbandschleifmaschine der Firma Bütfering, Herzebrock-Clarholz, Typ OPTIMATSCOOCE-DIAMOND vorbereitet, um eine optimale Oberfläche und gleiche Materialstärke zu gewährleisten.

\subsubsection{Analytische Messgeräte}

\section{$\mathrm{pH}-$ Meter}

Zur Bestimmung der $\mathrm{pH}-$ Werte der verschiedenen Furniere wurde ein Microprozessor pH-Meter der Firma WTW, Freiburg verwendet.

\section{Titrometer}

Die Ermittlung der Pufferkapazität des Holzes in mmol NaOH/100 g erfolgte unter Zuhilfenahme eines Titroprozessors der Firma Schott, Mainz (Modell Titroline Easy) in wässrigen Auszügen der Kalt- und Heißwasserextraktionen. 


\section{Universalprüfmaschine}

Zur Ermittlung der mechanisch-technologischen Eigenschaften des Werkstoffes und seiner Bestandteile diente eine Material-Tischprüfmaschine der Firma Zwick/Roell, Ulm (Modell T1-FR010TH.A50), mit einer maximalen Prüfkraft von $10 \mathrm{kN}$. Mit dieser Maschine wurden Biege-, Zug- und Abhebeversuche durchgeführt.

Die Maschinensteuerung und Versuchsauswertung erfolgte mit der Software „testXpert“ der Fa. Zwick/Roel, Ulm.

\section{Türprüfmaschine}

Die Dauerhaftigkeit des Werkstoffes hinsichtlich seiner Falzfestigkeit, also der maximalen Anzahl an Faltvorgängen, wurde mittels einer Türprüfmaschine der Firma Hegewald \& Peschke, Nossen durchgeführt.

Die Auswertung der Ergebnisse folgte mittels der Software „labcontrol“.

\section{$\underline{\text { Schneidmühle }}$}

Zur Herstellung extrahierfähigen Materials wurde eine Schneidmühle der Firma Retsch $\mathrm{GmbH}$, Haan verwendet. In dieser wurden die Furniere auf die zur Extraktion der Holzinhaltsstoffe benötigte Größe zerkleinert.

\section{$\underline{\text { 3D-Oberflächenmesstechnik }}$}

Zur Bestimmung der Rauheit von Furnieroberflächen wurde ein Tischgerät der Firma FRT GmbH, Bergisch Gladbach verwendet. Das Gerät wurde mit der Software "Acquire“ gesteuert, die Daten mit der Analysesoftware „Mark III“ ausgewertet. Beide Produkte stammen ebenfalls aus dem Hause FRT.

\subsection{Methoden}

Der in dieser Arbeit entwickelte Verbundwerkstoff „Faltfurnier“ wurde hinsichtlich bestimmter analytischer Parameter sowie auf seine mechanisch-technologischen Eigenschaften untersucht.

Während sich die analytischen Untersuchungen ausschließlich auf die verwendeten Furniere beziehen, wurden die mechanisch-technologischen Eigenschaften sowohl für einzelne Bestandteile des Werkstoffes (z. B. PVC-Folie) aber auch für Teilverbünde und den Gesamtverbund aus 5-Schichten untersucht.

In den folgenden Abschnitten werden die Vorgehensweisen für die analytischen und die mechanisch-technologischen Eigenschaften erläutert. 


\subsubsection{Analytische Untersuchungen}

Da es bei Verklebungen, vorwiegend bei extraktstoffreichen Hölzern wie dem der Eiche (vergl. Kapitel 2.2.1), zu Schwierigkeiten kommen kann, wurden Untersuchungen zu den Extrakstoffgehalten der verwendeten Furniere aus Erle und Eiche durchgeführt.

Da diese beiden Holzarten zu zwei verschiedenen Gruppen (vergl. Kapitel 3.1.1), nämlich den zerstreutporigen (Erle) und den ringporigen (Eiche) Laubhölzern, gehören, erfolgte zusätzlich eine Untersuchung der Topographie der Oberfläche beider Furnierarten, um einen möglichen Einfluss dieser auf die adhäsiven Eigenschaften des Verbundmaterials zu erforschen.

In den beiden folgenden Abschnitten wird die Vorgehensweise $\mathrm{zu}$ den o. g. Untersuchungen erläutert.

\subsubsection{Bestimmung des Extraktstoffgehaltes in Kaltwasser}

Zur Bestimmung des Exktraktstoffgehaltes in Kaltwasser wurden zunächst Furnierblätter aus Erle und Eiche mittels einer Schneidmühle zerkleinert und im Anschluss darrgetrocknet.

Das zu ca. $5 \mathrm{~g}$ abgemessene darrgetrocknete Probenmaterial wurde in einen Erlenmeyerkolben gegeben und $150 \mathrm{ml}$ bidest. Wasser hinzugefügt. Die Proben verblieben für $24 \mathrm{~h}$ bei Raumtemperatur auf dem Rüttler mit ca. 120 rpm.

Im Anschluss erfolgte die Filtrierung der Suspension mit gedarrten Fritten. Nach erneutem Darren wird der Exktraktstoffgehalt gravimetrisch gemessen (Ritter 2011). Der Exktraktstoffgehalt errechnet sich wie folgt:

$$
\text { Exktraktstoffgehalt }(\%)=\frac{\text { atro Einwaage }- \text { atro Auswaage }}{\text { atro Einwaage }} \times 100
$$

\section{Bestimmung des $\mathrm{pH}-$ Wertes}

Mittels einer $\mathrm{pH}$-Elektrode, die bei $20^{\circ} \mathrm{C}$ für 4 min direkt in der abfiltrierten Lösung $(20 \mathrm{ml})$ positioniert wird, kann der $\mathrm{pH}$-Wert bestimmt und am Gerät abgelesen werden.

\section{Bestimmung der Pufferkapazität}

Zur Bestimmung der Pufferkapazität werden $20 \mathrm{ml}$ der abfiltrierten Lösung mittels Titroprozessors mit 0,01 $\mathrm{M} \mathrm{NaOH}$ bis zum Erreichen der Neutralität versetzt. Anhand der verbrauchten Menge an Lauge, die bis zum Erreichen einer neutralen Lösung ( $\mathrm{pH}-$ Wert 7) nötig ist, kann die Pufferkapazität ( $\mathrm{mmol} \mathrm{NaOH/100} \mathrm{g} \mathrm{atro} \mathrm{Probenmaterial)}$ errechnet werden. $1 \mathrm{ml}$ Lauge entspricht in diesem Fall 1,5 mmol NaOH/100 g atro Probenmaterial. 


\subsubsection{Bestimmung des Extraktstoffgehaltes in Heißwasser}

Die Bestimmung der in heißem Wasser löslichen Bestandteile der Furniere erfolgte durch eine Heißwasserextraktion. Hierfür wurden $2 \mathrm{~g}$ atro Probenmaterial in einen Erlenmeyerkolben gegeben und mit $150 \mathrm{ml}$ bidest. Wasser versetzt. Der Ansatz wurde für $2 \mathrm{~h}$ auf einer Reihenheizbank unter Rückfluss mit Siedesteinen versetzt gekocht. Im Anschluss erfolgte das Abnutschen der Materialsuspension über gedarrten und gewogenen Fritten. Nach dem erneuten Darren der Fritten folgte die gravimetrische Bestimmung der Heißwasserextrakte analog zu den in kaltem Wasser löslichen Extrakten.

Die Bestimmung des pH-Wertes sowie der Pufferkapazität verläuft nach dem gleichen Schema wie in Kapitel 3.2.1.1 beschrieben, wobei $1 \mathrm{ml}$ Lauge hier 3,75 mmol NaOH/100g atro Probenmaterial entspricht.

\subsubsection{Bestimmung des Extraktstoffgehaltes mittels sukzessiver Extraktion}

Die quantitative Bestimmung der in den Furnieren erhaltenen Stoffklassen erfolgte durch eine sukzessive Extraktion im Soxhlet-Extraktor. Hierzu wurden $7 \mathrm{~g}$ atro bzw. $5 \mathrm{~g}$ atro Probenmaterial mit unterschiedlichen Lösungsmitteln steigender Polarität im Soxhlet-Extraktor unter Rückfluss extrahiert. Das Probenmaterial wird mit jeweils $200 \mathrm{ml}$ des Lösungsmittels in gedarrte Rundkolben gegeben und jeweils $6 \mathrm{~h}$ extrahiert. Nach jedem Durchgang werden die Kolben mit den extrahierten Kolben eingedampft, gedarrt und gewogen.

Tabelle 3-1: Exktraktionsstufen und zugehöriges Lösungsmittel.

\begin{tabular}{|c|l|}
\hline Extraktionsstufe & $\begin{array}{l}\text { Lösungsmittel } \\
\text { (Mischungsverhältnis) }\end{array}$ \\
\hline 1 & Petrolether \\
\hline 2 & Diethylether \\
\hline 3 & Aceton/ Wasser $(9: 1)$ \\
\hline 4 & Ethanol/ Wasser $(4: 1)$ \\
\hline 5 & Wasser $\left(60{ }^{\circ} \mathrm{C}\right)$ \\
\hline
\end{tabular}

Die verschiedenen Extraktionsstufen mit den zugehörigen Lösungsmitteln finden sich in Tabelle 3-1. 


\subsubsection{Bestimmung der Oberflächenrauigkeit}

Um einen möglichen Einfluss der Oberflächenstruktur der Furniere auf die adhäsiven Eigenschaften zu überprüfen, wurden an Proben von Erlen- und Eichenfurnieren Messungen der Oberflächenrauigkeit vorgenommen. Abbildung 3-1 zeigt die Prüfeinrichtung zur Messung der Rauigkeit von Oberflächen.

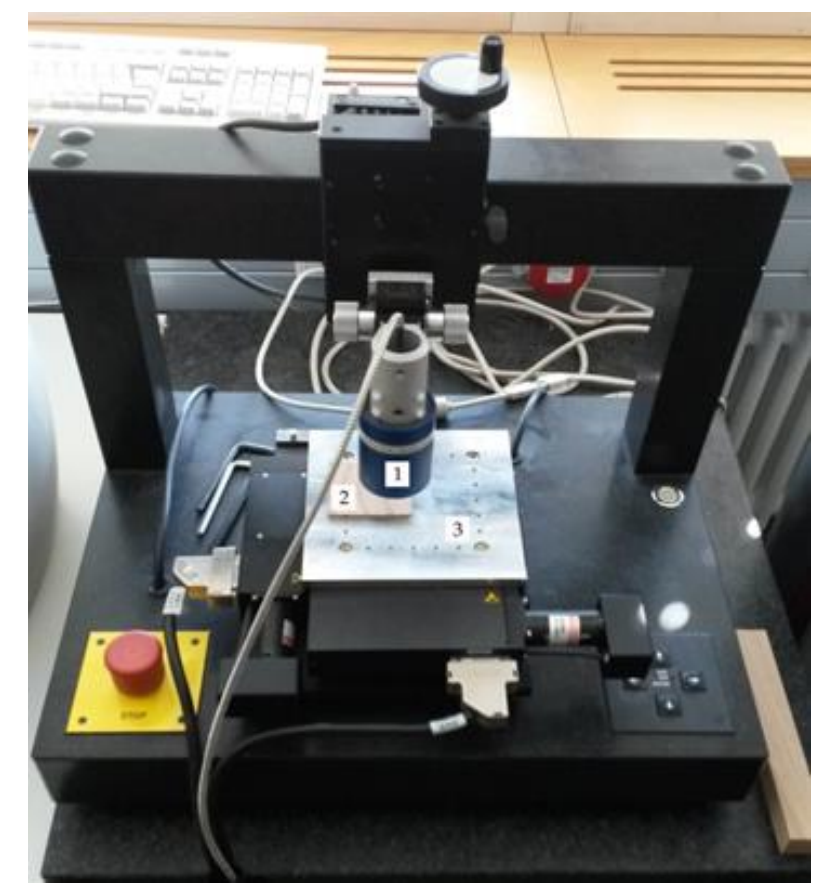

Abbildung 3-1: Micro Prof mit Erlenfurnierprobe bestückt. 1- Optischer Messkopf, 2- Probekörper aus Erlenfurnier und 3- Beweglicher Prüftisch.

Die Bestimmung der Rauigkeit erfolgt mittels eines fest positionierten optischen Messkopfes. Der Prüfkörper wird auf einem Probentisch platziert, der sich auf der xund y-Achse bewegt. Abbildung 3-2 zeigt den für die Untersuchungen ausgewählten Interessensbereich sowie den Fahrweg des Tisches für die Messungen.

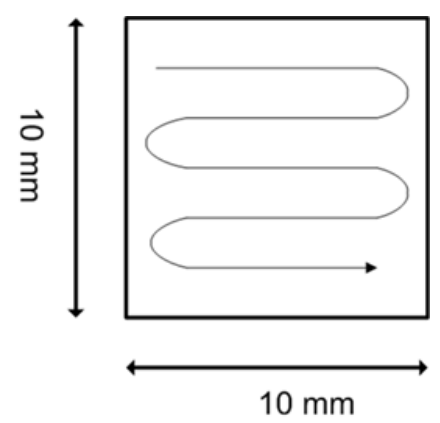

Abbildung 3-2: Interessensbereich und Fahrweg des Messkopfes für die Bestimmung der Oberflächenrauigkeit der verwendeten Furniere.

Die gewählte Auflösung für diesen Bereich betrug 500 Linien mit je 500 Messpunkten. Der Abstand zwischen den Messpunkten bezifferte sich somit auf $20 \mu \mathrm{m}$. Je Probe wurden 20 min Prüfzeit benötigt. 


\subsubsection{Bestimmung der mechanisch technologischen Eigenschaften}

Mechanisch-technologische Eigenschaften stehen zumeist im Vordergrund bei der Entwicklung von Werkstoffen. Wie in Kapitel 1.2 und Kapitel 2.7.1 bereits aufgezeigt. Auch für den in dieser Arbeit entwickelten Verbundwerkstoff Faltfurnier wurden Anforderungen und Zielgrößen an dessen Eigenschaften definiert und anhand der in den folgenden Abschnitten erläuterten Materialprüfungen untersucht.

\subsubsection{Normvorgaben zur Prüfung von Faltfurnier}

Da es sich bei Faltfurnier um einen neuen Werkstoff handelt, der in dieser Art bisher nicht auf dem Markt existent ist, sind die aktuell etablierten Normen für Holz- und Holzwerkstoffe nur partiell oder gar nicht anwendbar. Teilweise existieren keine Normen für dieses Halbzeug, die eine Prüfung der an das Faltfurnier gestellten Anforderungen darstellen können. Aus diesem Grund mussten die angewandten Normen z. T. modifiziert resp. aus anderen industriellen Sektoren wie beispielsweise der Möbelindustrie herangezogen werden.

\subsubsection{Vorabprüfung mittels Prüfmuster}

Um eine erste Begutachtung der adhäsiven Eigenschaften neuer Materialkombinationen resp. der funktionalen Eigenschaften der verwendeten Klebstoffe durchzuführen, wurde ein eigens entwickeltes "Prüfmuster" in Form eines symmetrischen Rasters mittels Formatkreissäge in den erstellten Werkstoff gesägt (siehe Abbildung 3-3).

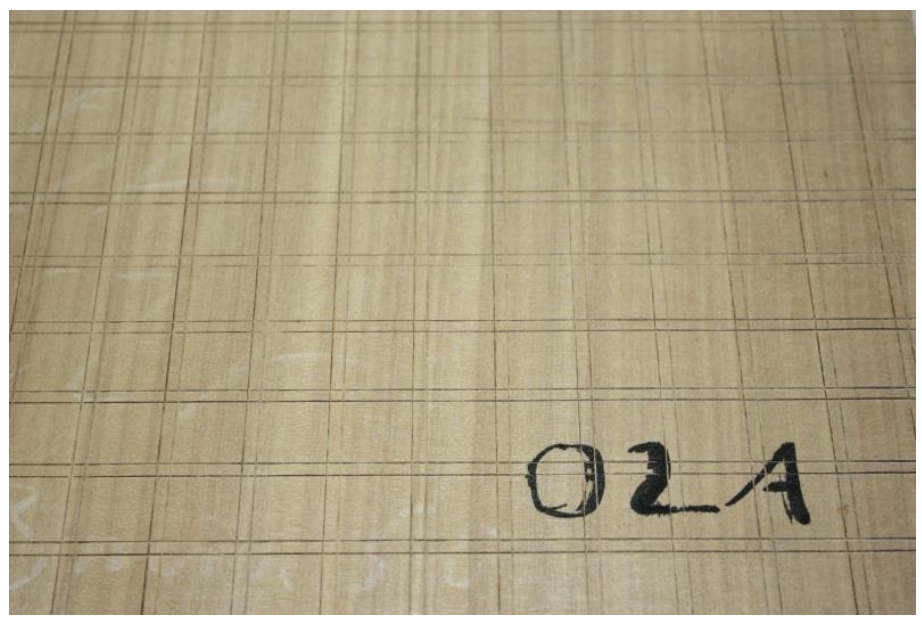

Abbildung 3-3: Prüfmuster zur ersten Kontrolle der adhäsiven und kohäsiven Eigenschaften des Materialverbundes.

$\mathrm{Zu}$ diesem Zweck wurde das Sägeblatt auf einen Winkel von $45^{\circ}$ Neigung eingestellt und die Höhe des Sägeblattes an die Furnierdicke angepasst, so dass eine Beschädigung der Mittelschicht ausgeschlossen werden konnte. 
Durch das Prüfmuster (siehe Abbildung 3-3) konnte eine erste Beurteilung der Adhäsion erfolgen, indem das Material händisch gefaltet und währenddessen am Punkt der höchsten Spannung versucht wurde, einzelne Segmente des Furniers von der Mittelschicht zu lösen.

Konnten bei diesem Prozess ungenügende Festigkeiten konstatiert werden (einzelne Segmente ließen sich problemlos lösen), folgten keine aufwendigen (im Folgenden beschriebene) Abhebefestigkeitsversuche sondern die Herstellung neuer Materialkombinationen.

\subsubsection{Probenahme, Zuschnitt und Überwachung (Deutsche Norm DIN EN 326-1)}

Die Auswahl der Anzahl und der Lage von Probekörpern aus Faltfurnierplatten geschah nach DIN EN 326-1. Entsprechend der Vorgaben wurden für die Biegefestigkeitsversuche je Faltfurnierplatte 6 Prüfkörper mit parallelem Faserverlauf des Deckfurniers zur Prüfachse sowie 6 Prüfkörper mit quer zur Prüfachse verlaufenden Fasern des Deckfurniers ausgewählt.

Zur Bestimmung der Abhebefestigkeit wurden jeweils 8 Probekörper ausgewählt, die von der Oberseite der Faltfurnierplatte stammten sowie 8 Prüfkörper von der Unterseite. Ober- und Unterseite definieren sich über die PVC-Folie, da diese auf einer Seite strukturiert und auf der gegenüberliegenden Seite unstrukturiert ist. Die strukturierte Seite (siehe Abbildung 3-4 links) ist als Oberseite festgelegt.

Weiterhin erfolgte entsprechend der o. g. Norm die Probenahme von jeweils 6 bzw. 8 Probekörpern vom Plattenrand sowie 6 bzw. 8 Probekörpern aus der Plattenmitte.

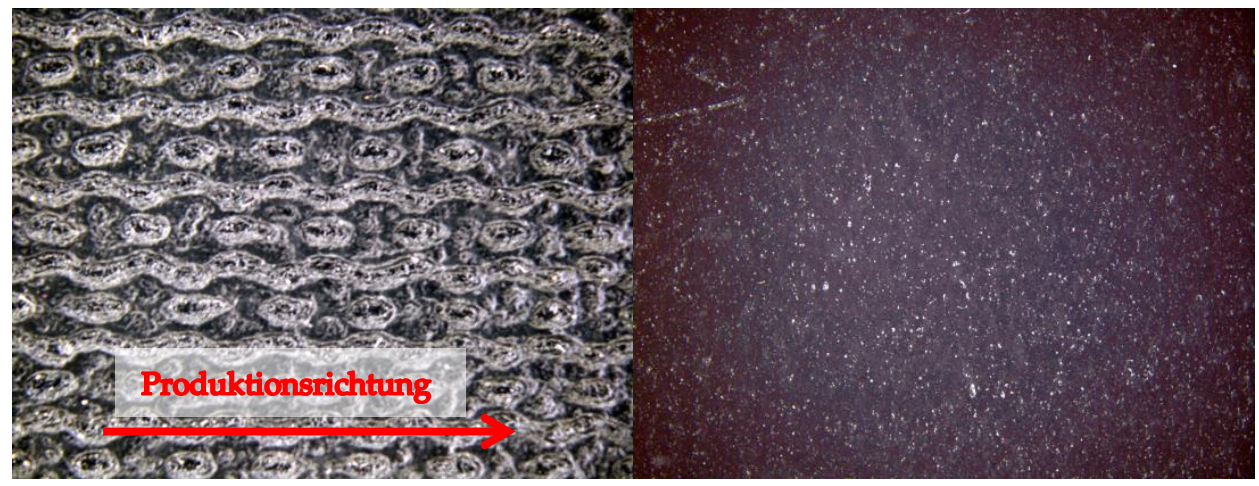

Abbildung 3-4: Fotografie der Oberfläche der PVC-Folie bei 10-facher Vergrößerung mittels Auflichtmikroskop. Strukturierte Seite (links) und unstrukturierte Seit (rechts). (Aufnahme: Peter Werndl)

Die folgende Tabelle 3-2 gibt einen Überblick über die Lage und Anzahl der entnommenen Probekörper. 
Tabelle 3-2: Übersicht über die Lage und Anzahl der Probekörper je Versuch.

\begin{tabular}{|c|c|c|c|c|c|c|c|c|}
\hline & \multicolumn{3}{|c|}{ Abhebefestigkeit } & \multicolumn{3}{|c|}{ Biegefestigkeit } & \multicolumn{2}{|c|}{ Zugspannung } \\
\hline & $\begin{array}{l}\text { Platten- } \\
\text { seite }\end{array}$ & $\begin{array}{l}\text { Lage } \\
\text { in der } \\
\text { Platte }\end{array}$ & $\begin{array}{l}\text { Anzahl } \\
\text { Probe- } \\
\text { körper }\end{array}$ & $\begin{array}{l}\text { Platten- } \\
\text { seite }\end{array}$ & $\begin{array}{l}\text { Faserverlauf } \\
\text { der } \\
\text { Deckschicht } \\
\text { zur } \\
\text { Prüfrichtung }\end{array}$ & $\begin{array}{l}\text { Anzahl } \\
\text { Probe- } \\
\text { körper }\end{array}$ & $\begin{array}{l}\text { Lage zur } \\
\text { Produktion } \\
\text { s-richtung }\end{array}$ & $\begin{array}{l}\text { Anzahl } \\
\text { Probe- } \\
\text { körper }\end{array}$ \\
\hline & oben & Mitte & 4 & oben & parallel & 6 & parallel & 5 \\
\hline & oben & Rand & 4 & unten & senkrecht & 6 & senkrecht & 5 \\
\hline & unten & Mitte & 4 & & & & & \\
\hline & unten & Rand & 4 & & & & & \\
\hline Summe & & & 16 & & & 12 & & 10 \\
\hline
\end{tabular}

Für die Überprüfung der Zugspannung der Mittelschicht wurden gemäß Deutsche Norm DIN EN ISO 527-1 jeweils 5 Probekörper parallel und 5 Probekörper senkrecht zur Produktionsrichtung entnommen. Die Produktionsrichtung konnte anhand von mikroskopischen Aufnahmen bestimmt werden, die vorab an Proben jeder Folienrolle erfolgten (siehe Abbildung 3-4 links).

\subsubsection{Abhebefestigkeit der Oberfläche Deutsche Norm DIN EN 311}

Verlief der „Vorversuch“ positiv, wurden zur Verifizierung und Quantifizierung der adhäsiven Eigenschaften des Materialverbundes Abhebefestigkeitsversuche durchgeführt.

Dies erfolgte mit leichter Modifikation nach den Vorgaben der DIN EN 311 (2002). Zu Herstellung der Prüfkörper werden die Faltfurnierplatten wechselseitig auf eine auf 17,50 mm kalibrierte Multiplexplatte aufgeklebt (mittels PVAc-Leim). An diesem Punkt erfolgte die Modifikation. Die Norm schreibt bei Prüfmaterialdicken unter $15 \mathrm{~mm}$, die in diesem Falle vorliegen, eine Versteifung durch eine Stahlplatte von mindestens $10 \mathrm{~mm}$ vor (vergl. Deutsche Norm DIN EN 311).

Die vorgegebenen Stahlplatten wurden ersetzt, da eine Verklebung der Probekörper auf dem Träger mit Heißkleber nicht möglich war.

Die Verleimung der Probekörper erfolgte vor dem Zuschnitt. Der Grund hierfür ist eine sauberere Schnittführung ohne Ausfransen des Probekörpers sowie die Vermeidung von Vorschädigungen durch beim Sägen entstehende Vibrationen.

Nach der Verleimung von Faltfurnierplatten auf die Multiplexplatte wurden die Probekörper mit Kantenmaßen von 50 mm x 50 mm produziert.

Die Abhebefestigkeit errechnet sich mit der nachfolgenden Gleichung:

$$
\text { Abhebefestigkeit der Oberfläche }=\frac{\text { Höchstkraft }(N)}{\text { Oberfläche }\left(\mathrm{mm}^{2}\right)}
$$

Sie wird in $\mathrm{N}^{*} \mathrm{~mm}^{-2}$ angegeben (Deutsche Norm DIN EN 311). 
3.2.2.5 Bestimmung des Biege-Elastizitätsmoduls und der Biegefestigkeit (DIN EN 310)

Die Prüfung der Biegefestigkeit erfolgte nach der Deutsche Norm DIN EN 310.

Durch das mittige Aufbringen einer Last auf einen an zwei Punkten aufliegenden Prüfkörper erfolgt die Ermittlung des Biegeelastizitätsmoduls sowie der Biegefestigkeit.

Die Biegefestigkeit ergibt sich aus der Ermittlung des Verhältnisses von Biegemoment $\mathrm{M}$ bei Bruchlast $\mathrm{F}_{\max }$ zum Widerstandsmoment seines vollen Querschnittes (Deutsche Norm DIN EN 310).

Die Biegefestigkeit wird in $\mathrm{N}^{*} \mathrm{~mm}^{-2}$ angegeben und errechnet sich nach folgender Formel:

$$
\begin{array}{ll}
\quad \text { Biegefestigkeit in } N * \mathrm{~mm}^{-2}=\frac{3 * F_{\text {max }} * l_{1}}{2 * b * t^{2}} \\
F_{\text {max }} \quad=\text { Bruchkraft in } N \\
l_{1} \quad=\text { Stützweite in } \mathrm{mm} \\
b \quad=\text { Breite des Prüfkörpers in mm } \\
t \quad=\text { Dicke des Prüfkörpers }
\end{array}
$$

Der E-Modul wird nach Norm in $\mathrm{N}^{*} \mathrm{~mm}^{-2}$ angegeben. Im Folgenden Ergebnisteil wird dieser jedoch zur besseren Übersicht in $\mathrm{kN}^{*} \mathrm{~mm}^{-2}$ aufgeführt. Er errechnet sich nach Deutsche Norm DIN EN 310 mit nachstehender Formel:

$$
E_{m}=\frac{l_{1}^{3} *\left(F_{2}-F_{1}\right)}{4 * b * t^{3}\left(a_{2}-a_{1}\right)}
$$

$l_{1} \quad=$ der Abstand zwischen den Mitten der Auflager, in $\mathrm{mm}$

$b \quad=$ Prüfkörperbreite in $\mathrm{mm}$

$t \quad=$ Prüfköperdicke in $\mathrm{mm}$

$F_{2}-F_{1}=$ Kraftzunahme $m$ geradlinigen Bereich des Kraft-Durchbiegungs-

.Diagrammes, in N. F1 muss ungefähr $10 \%$ und F2 ungefähr $40 \%$ .der Bruchkraft betragen

$a_{2}-a_{2}=$ Durchbiegungszunahme in Prüfkörpermitte (entsprechend $F_{1}-F_{2}$ )

\subsubsection{Bestimmung der Zugeigenschaften Deutsche Norm DIN EN ISO 527-3}

Die Zugfestigkeit der Mittelschicht wurde nach DIN EN ISO 527-3 unter Berücksichtigung von DIN EN ISO 527-1 ermittelt.

Durch das Dehnen des an den zwei langen Enden eingespannten Probekörpers mit kontinuierlicher Geschwindigkeit entlang seiner größten Hauptachse, bis hin zum Bruch, wird die Zugfestigkeit bestimmt. 
Als Probekörperform wurde die in DIN EN ISO 527-3 als Typ 2 beschriebene Form eines Rechteckes verwendet. Die Maße betrugen hierbei $b=20 \mathrm{~mm}$ und $\mathrm{l}_{3}=200 \mathrm{~mm}$.

Die Zugspannung errechnet sich laut Deutsche Norm DIN EN ISO 527-1 nach folgender Formel:

$$
\begin{gathered}
\text { Zugspannung in } N * \mathrm{~mm}^{-2}=\frac{F}{A} \\
F \quad=\text { die betreffende gemessene Kraft in } N \\
A \quad=\text { die Anfangsquerschnittsfläche des Probekörpers in } \mathrm{mm}^{2}
\end{gathered}
$$

\subsubsection{Modifizierte Bestimmung der Zugspannung}

Aufgrund diverser hergestellter Produktprototypen aus Faltfurnier (Aktenmappen, Wasserträger, etc.) konnte festgestellt werden, dass nicht nur eine Bestimmung der Zugspannung nach Deutsche Norm DIN EN ISO 527-3, die eine Prüfung entlang der längsten Hauptachse eine Probekörpers vorschreibt von Interesse ist, sondern auch die Zugspannung des Materials, insbesondere im $90^{\circ}$ Winkel, aber auch im Winkel von $45^{\circ}$. Dies basiert auf der Formgebung einiger zu erwartender Produktgruppen wie z. $\mathrm{b}$. einem Wasserträger (siehe Anhang). Die Hauptspannung tritt dort nicht in der geraden Achse sondern im angewinkelten Zustand auf. Um diese Belastungsgrenzen $\mathrm{zu}$ untersuchen, wurde im Rahmen einer Masterarbeit (vergl. Werndl 2016) eine Einspannvorrichtung entwickelt, die modular an der genutzten Prüfvorrichtung zu befestigen ist und die zu prüfenden Probekörper rutschfest einspannt (siehe Abbildung 3-5).

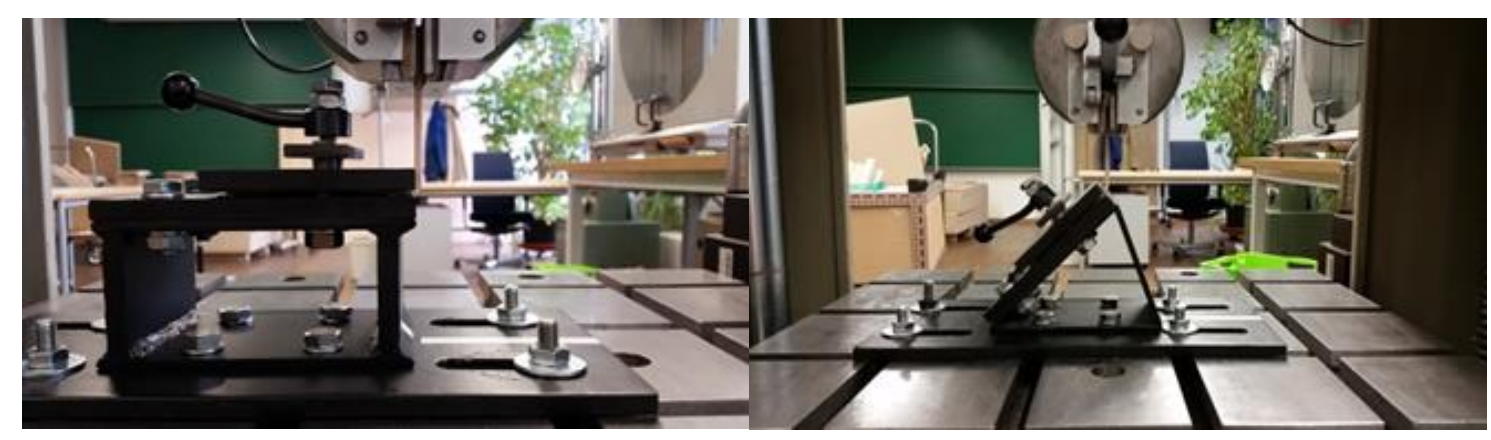

Abbildung 3-5: Einspannvorrichtung zur Zugspannungsprüfung im $90^{\circ}$ Winkel (links) und $45^{\circ}$ Winkel (rechts) montiert auf einer Universalprüfmaschine. (Foto: Peter Werndl)

Im Unterschied zur DIN EN ISO 527-3 wurde nicht nur die PVC-Folie sondern der ganze Materialverbund geprüft. Die Maße der Probekörper entsprechen der Norm. Dies geschah aus der zwingenden Annahme, dass bei der beidseitig in die Furnierschichten bis auf die Mittelschicht eingefrästen Nuten eine Materialkante entsteht, die durch einen „einschneidenden“ Effekt zu einer reduzierten Zugspannung im Vergleich zum reinen PVC führt (vergl. Kapitel 4.2.3). Entsprechend wurden die Probekörper planparallel zur Fräskante eingespannt (vergl. Werndl 2016). 
3.2.2.8 Festigkeits- und Dauerhaftigkeitsuntersuchungen der Faltnut in Anlehnung an (ISO 5626)

Zur Ermittlung der Dauerhaftigkeit der Mittelschicht des Werkstoffes bzw. der maximalen Anzahl der möglichen Faltungen erfolgten Materialprüfungen in Anlehnung an ISO 5626 (Paper - Determination of folding endurance).

Die Dauerhaftigkeitsuntersuchungen fanden an einer Türprüfmaschine der Fa. Hegewald \& Peschke, Nossen stattt.

Hierzu wurden Probekörper mit einer Breite von $160 \mathrm{~mm}$, einer Länge von $350 \mathrm{~mm}$ und einer Materialstärke von durchschnittlich 3,09 $\mathrm{mm}$ auf eine eigens angefertigte Konstruktion gespannt (siehe Abbildung 3-6).

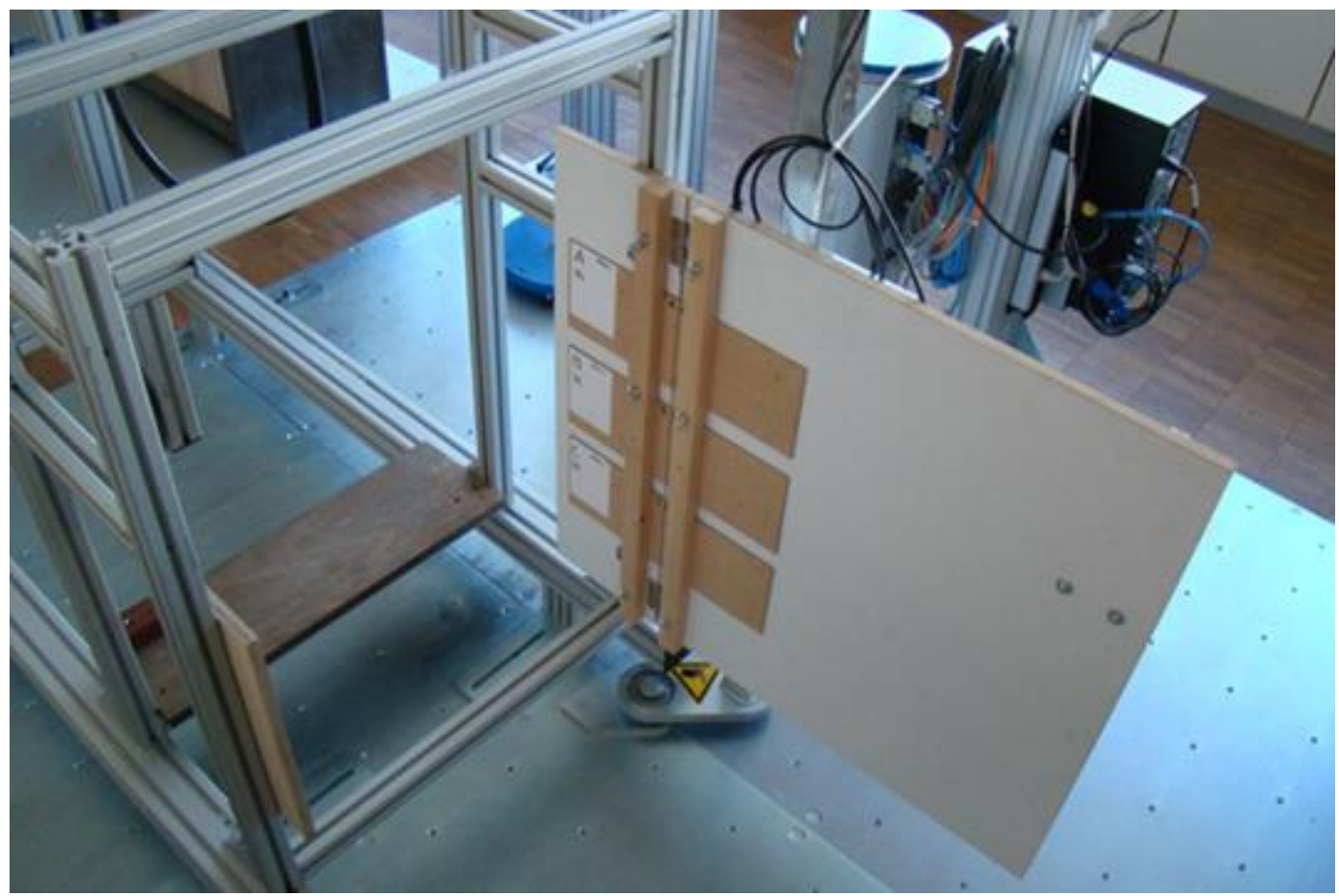

Abbildung 3-6: Türprüfmaschine mit eigens angefertigter Prüfvorrichtung (Foto: Peter Werndl).

Als Einspannvorrichtung dienten eine schmale sowie eine breite beschichtete MDF, die zur Fixierung der Prüfkörper mit zwei mittels Flügelschrauben zu befestigenden Massivholzleisten versehen waren. Die Massivholzleisten wurden $300 \mathrm{~mm}$ neben dem „Türspalt“ positioniert. Somit wurde eine ausreichende Befestigung der Prüfköper gewährleistet und ein Einfluss auf die zu prüfenden Faltnut vermieden.

Um die Vorrichtung in ihrer Vertikalachse $\mathrm{zu}$ fixieren und mögliche Horizontalverschiebungen $\mathrm{zu}$ vermeiden, wurden zusätlich zwei handelsübliche Scharniere angebracht.

Wesentliche Voraussetzung für eine fehlerfreie Prüfung ist die exakt senkrechte Positionierung der Faltnuten zueinander sowie im Spalt der Prüfvorichtung (Werndl 2014). 
Zur Prüfung der Probekörper wurde die Türprüfmaschine von der Position $0^{\circ}$ in die Position $90^{\circ}$ bewegt. Um eine Verfälschung der Daten durch Materialerwärmung zu vermeiden, belief sich die Zeitspanne je Bewegung auf $5 \mathrm{~s}$, ergänzt um eine Pause von 0,2 s nach jedem Bewegungszyklus. Die Prüfparameter für die Steuersoftware finden sich in Tabelle 3-3 (Werndl 2014).

Tabelle 3-3: Prüfparameter für Dauerhaftigkeitsversuche.

\begin{tabular}{|l|c|c|}
\hline \multicolumn{1}{|c|}{ Parameter } & Wert & Einheit \\
\hline Winkelgeschwindigkeit & 45 & $\% / \mathrm{s}$ \\
\hline Winkelbeschleunigung & 85 & $\% / \mathrm{s}^{2}$ \\
\hline Startwinkel & 0 & $\circ$ \\
\hline Endwinkel & -90 & $\circ$ \\
\hline Haltezeit & 0,2 & $\mathrm{~s}$ \\
\hline
\end{tabular}

Um eine gleichmäßige Belastung zu simulieren wurden die Prüfkörper nach 2000 Faltintervallen gewendet. Ein Zyklus/Intervall entspricht einer Bewegung von der Position $0^{\circ}$ in die Position $90^{\circ}$.

Eine Überprüfung auf Beschädigungen der MS erfolgte nach jeweils 200 Zyklen.

Als Kriterium des Materialversagens und damit einhergehender Zeitpunkt des Versuchsabbruchs wurde eine Rissbildung von $>20$ mm Länge festgelegt (Werndl 2014).

\subsubsection{Lagerung im starken Wechselklima}

Um die Formstabilität sowie die adhäsiven Eigenschaften des Materialverbundes auch außerhalb des normativen Klimas zu überprüfen und ungünstige Wettersituationen z. B. beim Transport oder falscher Lagerung zu simulieren, wurden Faltfurnierplatten mit den Maßen 750 mm x 350 mm in einer Klimakammer der Hochschule Rosenheim starkem Wechselklima ausgesetzt.

Die Lagerung der Proben erfolgte über einen Zeitraum von 3 Monaten bei den aus Tabelle 3-4 ersichtlichen Bedingungen.

Tabelle 3-4: Soll-Klima während der Versuchsperiode.

\begin{tabular}{|l|c|c|}
\hline Zeitraum & $\begin{array}{c}\text { Temperatur } \\
\left({ }^{\circ} \mathbf{C}\right)\end{array}$ & $\begin{array}{c}\text { Luftfeuchtigkeit } \\
(\mathbf{\%})\end{array}$ \\
\hline $01.10 .2013-15.10 .2013$ & 20 & 65 \\
\hline $15.10 .2013-21.11 .2013$ & 5 & 90 \\
\hline $21.11 .2013-12.12 .2013$ & 50 & 90 \\
\hline $12.12 .2013-07.01 .2014$ & 50 & 10 \\
\hline $07.01 .2014-10.01 .2014$ & 20 & 65 \\
\hline
\end{tabular}

Im Anschluss an die Lagerung in der Klimakammer wurden die Platten optisch bewertet. Hierzu fand eine Untersuchung der Probekörper auf etwaige Verformungen 
(Schüsselungen, Torsionen u. ä.) sowie auf Schäden im Furnier (Risse, Ablösungen etc.) statt.

\subsubsection{Herstellung des Verbundwerkstoffes}

Der im Zusammenhang mit dieser Dissertation entwickelte Verbundwerkstoff besteht aus zwei verschiedenen Materialien, einer flexiblen Mittelschicht und Furnierblättern, die miteinander verklebt werden. In den folgenden Kapiteln wird der Aufbau, die Anordnung der Schichten sowie der Herstellungsprozess erläutert.

\subsubsection{Aufbau des Verbundwerkstoffes}

Der Schichtverbundwerkstoff Faltfurnier besteht, die Klebschichten ausgenommen, aus 5 Werkstoffschichten. Die mittlere Lage bildet eine flexible Mittelschicht (MS). Diese wird beidseitig mit jeweils zwei Furnierblättern versehen. Die erste Lage Furniere wird auf beiden Seiten der MS mit parallelem Faserverlauf verklebt. Diese erste Furnierlage wird mit einem weiteren Furnierblatt, mit einem um $90^{\circ}$ verdrehten Faserverlauf beklebt (siehe Abbildung 3-7). Die erste Furnierschicht bezeichnet man auch als Absperrfurnier. Diese sorgt für die Formstabilität der Platte zur Vermeidung von Verwerfungen, Schüsselungen, Rissen u. ä. Fehlern, die durch Quellen und Schwinden entstehen können (vergl. hierzu Tsoumis 2009).

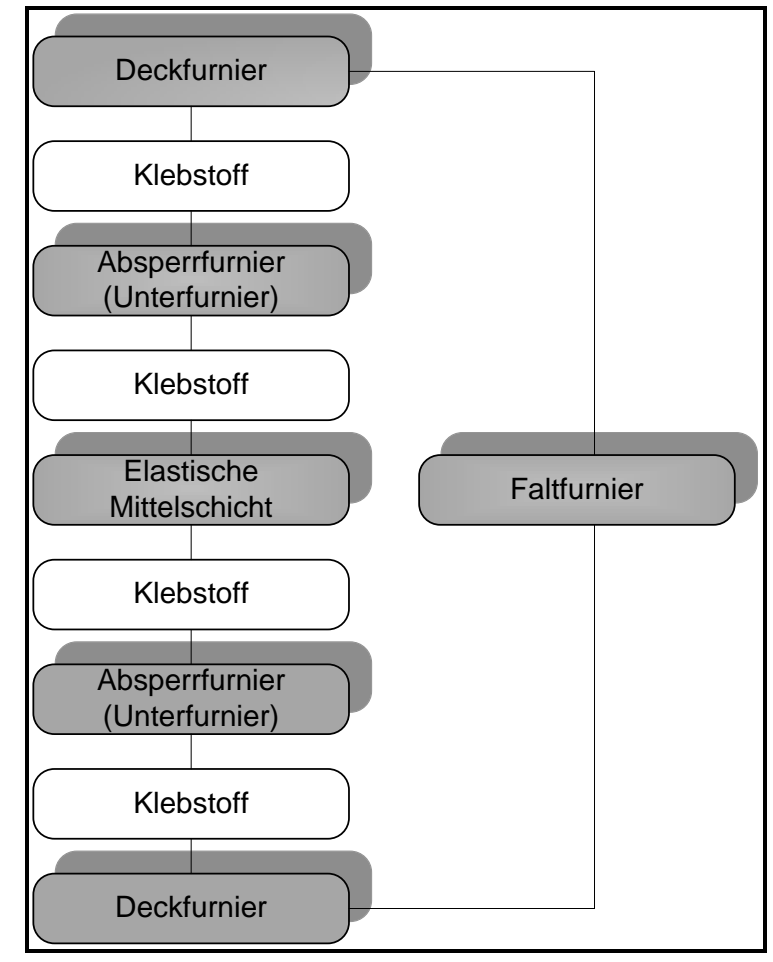

Abbildung 3-7: Schematische Darstellung des generellen Aufbaus des Verbundwerkstoffes.

Die Auswahl der Holzart für die Deckschicht ist hierbei unerheblich, da lediglich die Absperrschicht derart gewählt werden soll, dass diese sowohl mit dem Klebstoff als 
auch mit der MS eine ausreichende Adhäsion erreichen muss. Hierzu sind negative Interaktionen zwischen den einzelnen Komponenten auszuschließen.

\subsubsection{Herstellungsprozess}

Die Herstellung des Verbundwerkstoffes erfolgte für sämtliche Prototypenplatten in zwei Schritten.

Zunächst erfolgte die Verklebung der Mittelschicht mit dem Absperrfurnier mittels Heißverpressen. Hierzu werden Furnier, Mittelschicht und Klebefolie (Collano 46.002) auf die gewünschte Größe zugeschnitten. Im Anschluss erfolgt die Herstellung von Materialverbund 1 (MV 1). Dazu werden die in Abbildung 3-8 (Materialverbund 1) gezeigten Komponenten kantenbündig in dargestellter Abfolge zwischen zwei Aluminiumpressplatten platziert und heiß verpresst.

Der hierbei entstandene MV 1 wird im Anschluss an den Pressprozess zur Abkühlung auf Raumtemperatur unter Pressblechen gelagert. Nach erfolgtem Abkühlen wird auf diesen PVAc-Leim mittels Zahnspachtel (Zahnweite 1,5 mm-2 mm) appliziert. Im Anschluss erfolgt das Fügen von MV 1 mit dem um $90^{\circ}$ zur Faserrichtung versetzten Deckfurnier. Nach der Beleimung und dem Belegen des MV 1 mit dem Deckfurnier wird der neu entstandene Materialverbund (MV 2) kalt verpresst. Nach dem Abbinden des PVAc-Leimes in der Presse kann das nun fertige Halbzeug Faltfurnier (MV 2) entnommen und sofort weiterverarbeitet werden (siehe Abbildung 3-8).

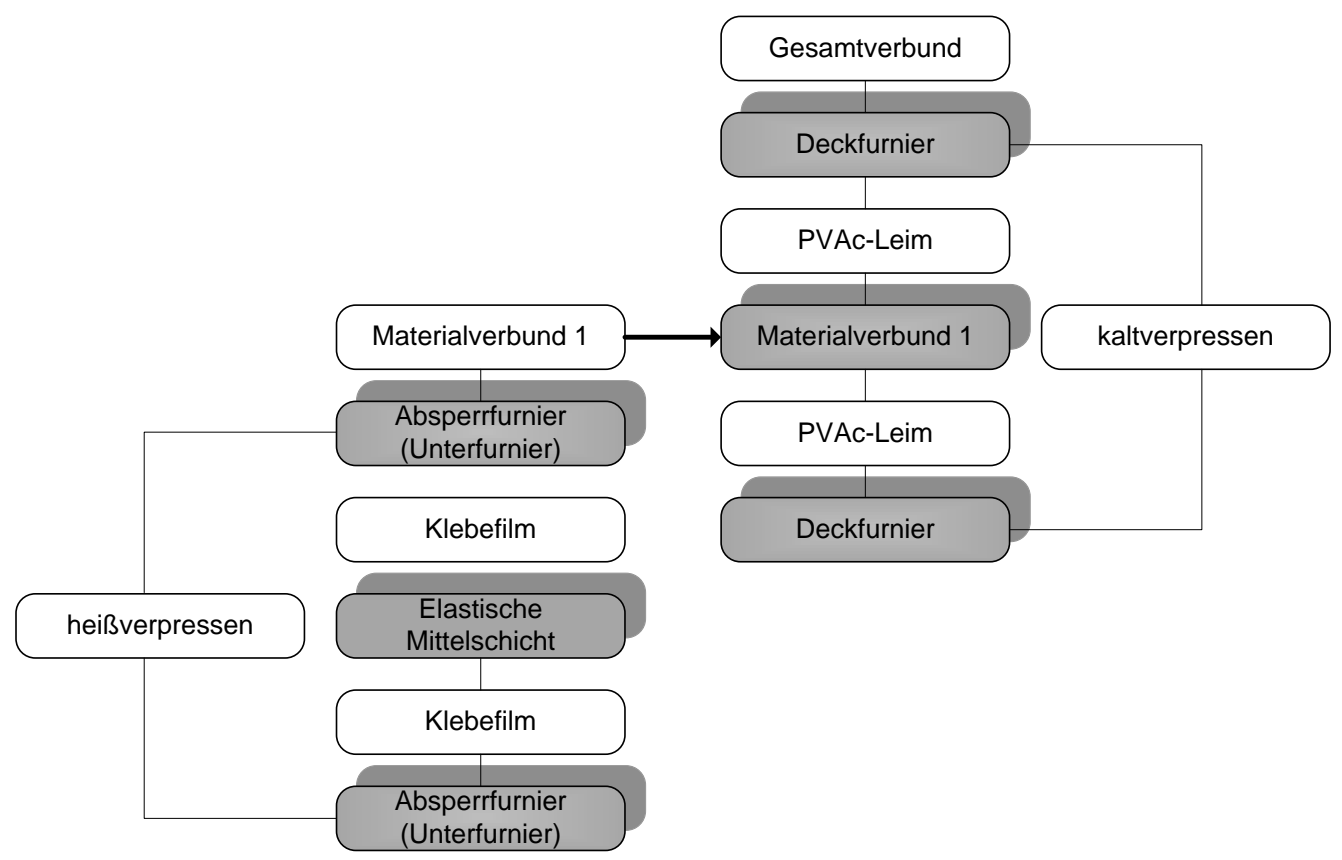

Abbildung 3-8: Schematische Darstellung des Herstellungsprozesses des Verbundwerkstoffes.

Wie sich im Laufe des Vorhabens herausstellte, ist das ganze Herstellungsverfahren auch mit einem einzigen Heißpressvorgang durchführbar. Hierzu wird auf die Verwendung von PVAc-Leim, wie in Abbildung 3-8 dargestellt, verzichtet und dieser durch den Klebefilm aus Pressvorgang 1 ersetzt. Die zu Versuchs- und Prüfzwecken 
hergestellten Faltfurnierplatten wurden jedoch, um die Vergleichbarkeit und Kontinuität zu wahren, entsprechen Abbildung 3-8 hergestellt. Die Verleimung der Deckschicht mit PVAc-Leim bietet zusätzlich den Vorteil, dass alle in dieser Arbeit zur Verwendung kommenden Furniere problemlos mit diesem Leim verklebt werden können.

\subsubsection{Statistische Auswertung der Daten}

Die statistische Auswertung der Daten der vorliegenden Arbeit wurde unter Zuhilfenahme der Statistiksoftware Origin Pro 8.5.0G SR1, der Firma OriginLab Cooperation, Northhampton, MA USA durchgeführt.

Der Ablauf der Auswertungen erfolgte stets nach gleichem Schema (vergl. Abbildung 3-9). Zunächst wurde jede Datenreihe mittels Shapiro-Wilk-Test auf Normalverteilung überprüft. Das Signifikanzniveau beträgt hierbei 0,05. Bei bestätigter Normalverteilung erfolgte im Anschluss eine Varianzanalyse mittels einfaktorielle-ANOVA. Im Rahmen der ANOVA bietet Origin zugleich die Möglichkeit eines Tests auf Varianzhomogenität nach Levene. Dieser Test wurde zeitgleich mit einem festgesetzten Signifikanzniveau von 0,05 durchgeführt. Der Mittelwertvergleich geschah mittels Tukey-Test.

Für den Fall, dass bei den Grundgesamtheiten keine Normalverteilungen und/oder eine Varianzheterogenitäten vorlagen, erfolgte eine nichtparametrische Analyse der Daten nach Kruskal-Wallis (Kruskal-Wallis-Test) mit einem Signifikanzniveau von ebenfalls 0,05 .

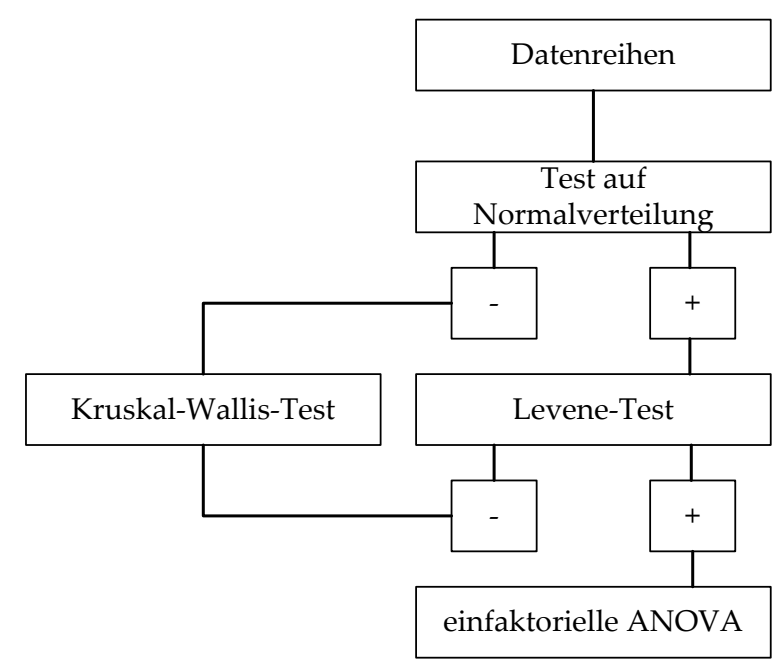

Abbildung 3-9: Schematische Darstellung des Ablaufes der statistischen Datenauswertung.

Die verwendete Software Origin bietet den Vorteil, dass das Auswertungsblatt sofort mittels Text das Testergebnis darstellt. 
Die statistischen Lage- und Streuparameter werden in dieser Arbeit häufig als Boxplots dargestellt.

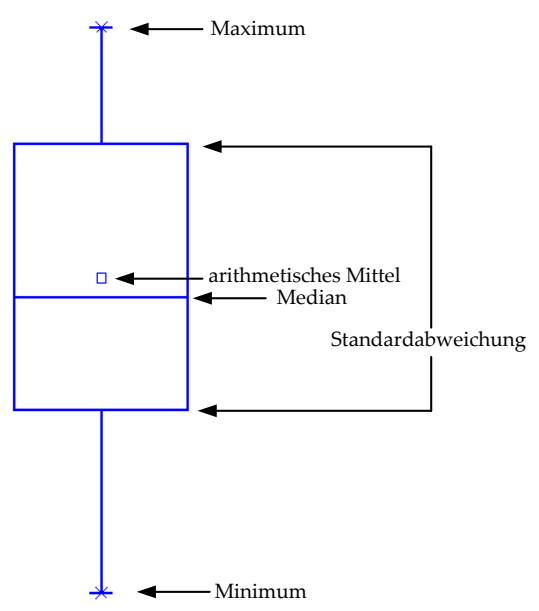

Abbildung 3-10: Aufbau der in dieser Arbeit verwendeten Boxplots zur Darstellung der statistischen Lage- und Streuparameter.

Abbildung 3-10 gibt einen Überblick zum Aufbau der verwendeten Boxplots. 


\section{Ergebnisse und Diskussion}

\subsection{Ausgangslage}

Ziel der vorliegenden Arbeit war es, einen Werkstoff auf Furnierbasis zu entwickeln, der mit einer flexiblen Mittelschicht ausgestattet ist, um durch symmetrisches, beidseitiges Einfräsen der Materialschichten aus Furnier einen Faltvorgang zu ermöglichen. Durch die gewählten Fräsmuster sollen somit verschiedenste Verpackungen, Behältnisse, Gebrauchsgegenstände $u$. ä. hergestellt werden können (vergl. Kapitel 1.2). Produktbeispiele hierzu finden sich im Anhang.

Um dieses Ziel zu erreichen, musste eine Mittelschicht gefunden werden, die den vorab definierten Anforderungen genügte sowie entsprechende Absperrfurniere, die zum einen kostengünstig sind und zum anderen mit dem Klebstoff und der Mittelschicht nicht in negative Wechselwirkungen treten. Weiterhin sollte die Mittelschicht leichte Beschädigungen durch den Fräskopf tolerieren können (vergl. Kapitel 1.2). Die Wahl fiel auf PVC-Folie, da Vorversuche mit anderen Materialien nur unbefriedigende Abhebefestigkeiten (als Maß für die Festigkeit/Qualität der Verklebung) erreichten. Als Beispiele seien die Werte der Abhebefestigkeiten von Erlenfurnier auf Filz $\left(\overline{\mathrm{x}}=0,29 \mathrm{~N}^{*} \mathrm{~mm}^{-2}\right)$ und Erlenfurnier auf Ethylen-Propylen-DienKautschuk $\left(\overline{\mathrm{x}}=0,18 \mathrm{~N}^{*} \mathrm{~mm}^{-2}\right)$ genannt.

Auch die Auswahl des Klebstoffes beruhte auf den Vorgaben der leichten Verarbeitbarkeit bei hoher Adhäsion - ohne negative Wechselwirkungen mit den jeweiligen Materialen - sowie eines möglichst geringen bis keinen Maschinenreinigungsbedarf. Als zu verwendende Klebstoffe wurde ein PU-basierter thermoplastischer Klebefilm (Collano 46.002) und ein Furnierleim auf PVAc-Basis (Kleiberit 322) ausgewählt.

Aufgrund der Vorgabe durch den beteiligten Projektpartner, die Firma Josef Wochner GmbHE Co. KG, Rosenfeld-Heiligenzimmern und die o. g. Aspekte wurde der Fokus auf Furniere aus Eiche und Erle gelegt.

\section{Herstellungsparameter der untersuchten Platten}

Der Schwerpunkt dieser Arbeit beschäftigte sich mit der Analyse der physikalischen, technischen und chemischen Eigenschaften des Werkstoffes und/oder seiner Bestandteile.

Als wesentliche Variable im Herstellungsprozess stellte sich die Presszeit heraus, auf der in der vorliegenden Arbeit ein besonderer Fokus liegt. 
Eine weitere Variable bei Verbundwerkstoffherstellung, die jedoch im Rahmen dieser Arbeit keinen wesentlichen Spielraum zulässt, ist die Presstemperatur.

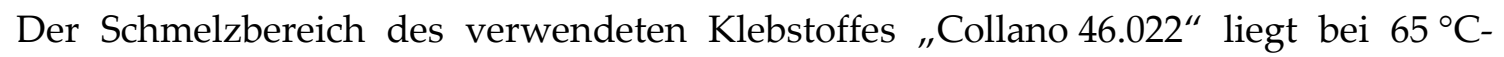
$85^{\circ} \mathrm{C}$, die minimale Klebfugentemperatur bei $90^{\circ} \mathrm{C}$ (Collano Adhesives AG 2011). Dementsprechend musste eine Temperatur im diesem Bereich gefunden werden.

Da bereits ab $100{ }^{\circ} \mathrm{C}$ Veränderungen im Holz stattfinden können, besonders die Festigkeitseigenschaften lassen mit zunehmender Temperatur nach (Fengel und Wegener 2003; Niemz und Sonderegger 2017; Kollmann 1955), sollte diese Temperaturgrenze nicht zu weit überschritten werden.

Weiterhin können ab $130^{\circ} \mathrm{C}$, zumindest bei längeren thermischen Einflüssen, erhöhte Farbveränderungen eintreten (Schneider 1966).

Zudem hat eine erhöhte Temperatur einen Einfluss auf die Eigenschaften der PVCFolie, wie z. B. auf die Glasübergangstemperatur und die Wärmedruckbeständigkeit. Die Wärmedruckbeständigkeit nimmt mit zunehmender Temperatur deutlich ab. Auch die Glasübergangstemperatur sinkt mit erhöhtem Weichmacheranteil (der durch den Hersteller nicht benannt wurde) deutlich (Diemert et al. 2005).

Um einen Kompromiss zwischen kurzer Presszeit (durch möglichst hohe Temperatur) und zugleich materialschonender Temperatur zu finden, wurde aus o. g. Gründen die Presstemperatur auf $120^{\circ} \mathrm{C}$ festgelegt

Die Verarbeitung des PVAc-Leims erfolgte bei Raumtemperatur, um einen zusätzlichen thermischen Einfluss auf den ersten Materialverbund aus PVC-Folie und Furnier zu vermeiden.

Ein weiterer, im Rahmen der vorliegenden Arbeit, wenig zu beeinflussender Herstellungsparameter ist der Pressdruck.

Die Herstellung der hier untersuchten Werkstoffverbünde erfolgte mit zwei verschiedenen Pressdrücken.

Sämtliche Verbindungen auf Basis des thermoplastischen Klebefilms „Collano 46.002" wurden mit einem Pressdruck von $13,96 \mathrm{~N}^{*} \mathrm{~cm}^{-2}$ verpresst.

Da dieser Klebstoff heiß verarbeitet wird, wirkt die Temperatur der Presse sich besonders auf die Schmelzviskosität aus. Um ein Herausdrücken des verflüssigten Klebstoffes aus der Klebefuge zu vermeiden, sollte der Pressdruck möglichst niedrig gewählt werden (Habenicht 2002). Ein einstellbarer Pressdruck von 10 bar der Niederdruckstufe der verwendeten Presse (vergl. Kapitel 3.1.4) liegt im unteren Bereich der Einstellmöglichkeiten.

Zudem sollte die PVC-Folie möglichst wenig gestaucht werden. Da der Werkstoffverbund bei $120^{\circ} \mathrm{C}$ verarbeitet wird und wie bereits erwähnt mit zunehmender Temperatur die Wärmedruckbeständigkeit der Folie stark nachlässt 
(Diemert et al. 2005), musste auch aus diesem Grund der Pressdruck möglichst gering gehalten werden.

Bei Verbindungen mittels PVAc-Leim wurde ein Pressdruck von $24,50 \mathrm{~N}^{*} \mathrm{~cm}^{-2}$ angewendet.

Einerseits gibt der Hersteller der Furnierpresse (vergl. 3.1.4) einen optimalen Druck für die jeweilige $\mathrm{zu}$ pressende Fläche vor, andererseits empfiehlt der Hersteller des verwendeten Klebstoffes „Kleiberit 322“ einen anzuwendenden Druck von 0,20 N*mm${ }^{2}$ bis $0,50 \mathrm{~N}^{*} \mathrm{~mm}^{-2}$ (KLEBCHEMIE M. G. Becker GmbH \& Co. KG 1999). Somit liegt der hier gewählte Pressdruck von gerundet $0,25 \mathrm{~N}^{*} \mathrm{~mm}^{-2} \mathrm{im}$ Bereich der Vorgaben.

\subsection{Werkstoffuntersuchungen der PVC-Folie}

Aufgrund diverser Vorversuche fiel die Wahl des Werkstoffes für die Mittelschicht auf eine PVC-basierte Teichfolie (Firma Heissner, Lauterbach) (vergl. Kapitel 3.1.2 und Kapitel 4.1).

Da je nach Endprodukt und durch die gewünschte Falteigenschaft eine Zugkraft auf die Folie wirkt, wurde die Zugfestigkeit der Folie unter verschiedenen Bedingungen untersucht, um die Belastungsgrenzen des Werkstoffverbundes zu bestimmen.

\subsubsection{Untersuchung der Zugfestigkeit unter Berücksichtigung der Produktionsrichtung}

Zur Ermittlung eines möglichen Einflusses der Extrusionsrichtung auf die Festigkeit der PVC-Folie, wurde eine Zugfestigkeitsprüfung an der Folie nach DIN EN ISO 527-3 durchgeführt. Die Bestimmung der Extrusionsrichtung der Probekörper erfolgte vor deren Zuschnitt mittels Mikroskop (vergl. Abbildung 3-4). Die Probekörper wurden parallel sowie senkrecht zur Herstellungsrichtung entnommen.

Tabelle 4-1: Ergebnisse der Zugfestigkeitsprüfung in unterschieden Lagen zur Extrusionsrichtung.

\begin{tabular}{|l|c|c|c|}
\hline Parameter & $\begin{array}{c}\text { Zugversuch } \\
\text { "parallel" } \\
\mathbf{n}=\mathbf{3 9} \\
{\left[\mathbf{N}^{*} \mathbf{m m}^{-2}\right]}\end{array}$ & $\begin{array}{c}\text { Zugversuch } \\
\text { "senkrecht" } \\
\mathbf{n}=\mathbf{3 9} \\
{\left[\mathbf{N}^{*} \mathbf{m m}^{-2}\right]}\end{array}$ & $\begin{array}{c}\text { Zugversuch } \\
\text { "gesamt" } \\
\mathbf{n}=\mathbf{3 9} \\
{\left[\mathbf{N}^{*} \mathbf{m m}^{-2}\right]}\end{array}$ \\
\hline Median Z & 14,16 & 11,74 & 12,90 \\
\hline Arithmetisches Mittel $\boldsymbol{\mu}$ & 14,19 & 11,72 & 12,96 \\
\hline Standardabweichung S & 0,23 & 0,19 & 1,26 \\
\hline Minimum & 13,76 & 11,21 & 11,21 \\
\hline Maximum & 14,69 & 12,05 & 14,69 \\
\hline
\end{tabular}


Wie Tabelle 4-1 zu entnehmen ist, beträgt die Zugfestigkeit parallel zur Extrusionsrichtung im arithmetischen Mittel $14,19 \mathrm{~N}^{*} \mathrm{~mm}^{-2}$ bei einer errechneten Standardabweichung von $0,23 \mathrm{~N}^{*} \mathrm{~mm}^{-2}$. Senkrecht zur Extrusionsrichtung konnte eine mittlere Zugfestigkeit von 11,72 $\mathrm{N}^{*} \mathrm{~mm}^{-2}$ gemessen werden. Die Standardabweichung beträgt $0,19 \mathrm{~N}^{*} \mathrm{~mm}^{-2}$. Somit liegt die Differenz der arithmetischen Mittelwerte der beiden Grundgesamtheiten bei 2,47 $\mathrm{N}^{*} \mathrm{~mm}^{-2}$. In der Gesamtbetrachtung differieren die Extremwerte bei einem Minimum von $11,21 \mathrm{~N}^{*} \mathrm{~mm}^{-2}$ und einem Maximum von $14,69 \mathrm{~N}^{*} \mathrm{~mm}^{-2}$ um 3,48 $\mathrm{N}^{*} \mathrm{~mm}^{-2}$. Die Extremwerte liegen in der Gesamtbetrachtung bei einem Minimum von 11,21 $\mathrm{N}^{*} \mathrm{~mm}^{-2}$ und einem Maximum von 14,69 $\mathrm{N}^{*} \mathrm{~mm}^{-2}$.

Auf Grundlage dieser Ergebnisse werden folgende Hypothesen aufgestellt:

$\mathrm{H}_{0}$ : Die jeweiligen arithmetischen Mittelwerte der Zugspannung, bezogen auf die Extrusionsrichtung „parallel“" und „senkrecht", unterscheiden sich nicht.

$\mathrm{H}_{1}$ : Die jeweiligen arithmetischen Mittelwerte der Zugspannung, bezogen auf die Extrusionsrichtung "parallel“ und „senkrecht", unterscheiden sich signifikant.

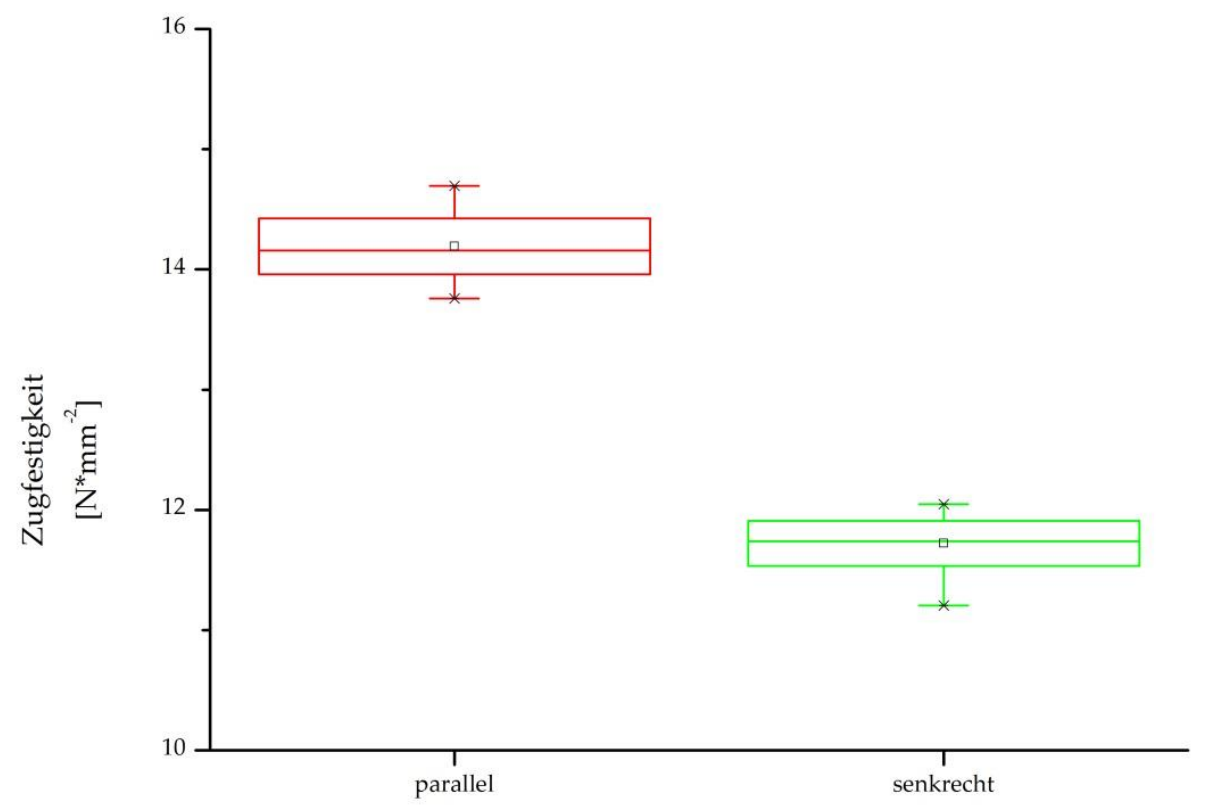

Abbildung 4-1: Boxplots zu den Versuchsergebnissen der Zugfestigkeitsprüfungen parallel und senkrecht zur Extrusionsrichtung.

Abbildung 4-1 zeigt, dass die Mittelwerte der Versuchsreihen „parallel“ und „senkrecht" bei jeweils geringer Standardabweichung stark auseinanderliegen. Da sich zudem die Maximal- bzw. Minimalwerte nicht „berühren“ ist davon auszugehen, dass die Mittelwerte beider Grundgesamtheiten signifikant differieren.

Aufgrund der gegebenen Ausgangsvorrausetzungen der Datenlage (Normalverteilung und Varianzhomogenität) erfolgt eine einfaktorielle Varianzanalyse (ANOVA). Das 
Ergebnis der ANOVA zeigt, dass sich bei einem Niveau von 0,05 die Mittelwerte der Grundgesamtheiten signifikant unterscheiden. Somit muss die $\mathrm{H}_{1}$-Hypothese angenommen und $\mathrm{H}_{0}$ verworfen werden.

\section{Diskussion der Versuchsergebnisse}

Die Zugfestigkeit der PVC-Folie liegt bei Messungen parallel zur Extrusionsrichtung mit einer Zugfestigkeit von $\bar{x}=14,19 \mathrm{~N}^{*} \mathrm{~mm}^{-2}$ ca. 17,41 \% höher als der Mittelwert der Zugfestigkeit in Höhe von $\bar{x}=11,72 \mathrm{~N}^{*} \mathrm{~mm}^{-2}$ bei senkrechter Lage.

Diesen Effekt beschreiben auch Simona (2016) und Retting (1975). Extrudierte Kunststoffe, zu denen die in dieser Arbeit verwendete PVC-Folie zählt (die Extrusion erfolgt vor dem Kalandrieren), besitzen eine s. g. Vorzugsrichtung. Diese ist bei extrudiertem Material in Extrusionsrichtung größer als senkrecht zu ihr. Dies bedeutet, dass die mechanischen Eigenschaften in Orientierungsrichtung größer als quer zu ihr sind. Somit verhalten sich extrudierte Folien anisotrop. Bei gepressten Kunststoffplatten hingegen liegt nahezu eine Isotropie vor, da keine Fließvorgänge in der Schmelze stattfinden (Simona 2016; Retting 1975).

Bei zukünftigen Endprodukten, die mit schweren Gewichten (z. B. ein Flaschenträger wie in Abbildung 4-2 dargestellt) beladen werden, ist es aufgrund der Datenlage empfehlenswert zunächst die Extrusionsrichtung der Folie zu bestimmen.

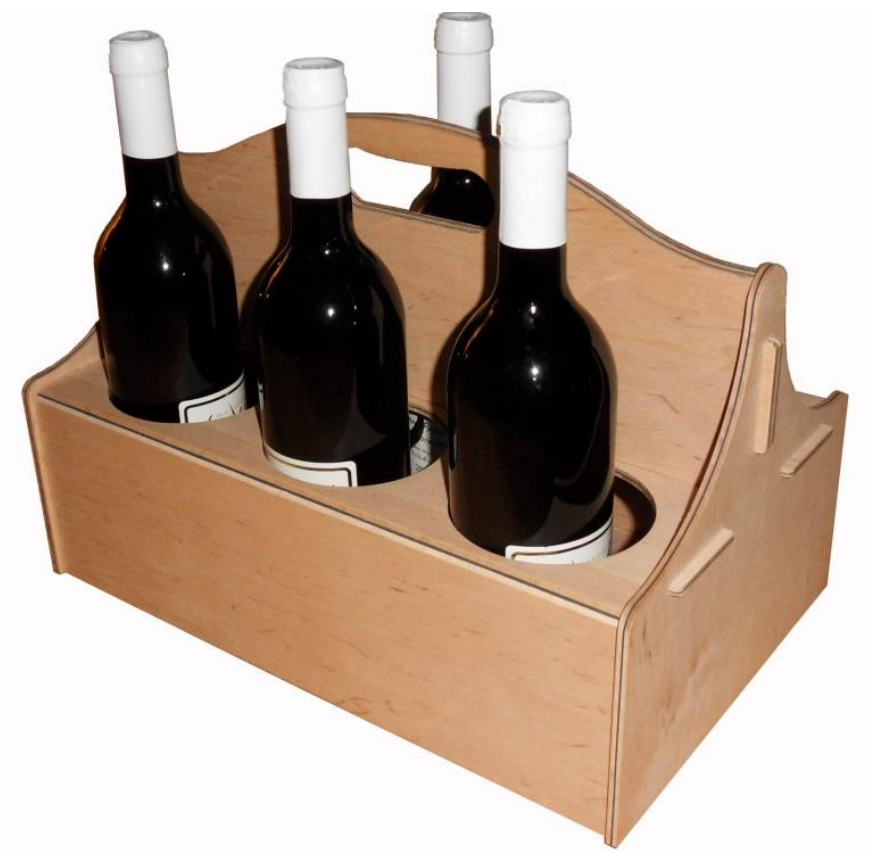

Abbildung 4-2: Flaschenträger aus Faltfurnier, bei dessen Fertigung die Extrusionsrichtung beachtet werden sollte.

Nach entsprechender Kennzeichnung des Halbzeuges sollte das Objektdesign und das dazugehörige „Fräslayout“ so gewählt werden, dass die Kanten des Objektes mit der höchsten Belastung parallel zur Extrusionsrichtung der Folie liegen. 


\subsubsection{Untersuchung der Zugfestigkeit bei verschiedenen Ausgangsbedingungen}

Während des Herstellungsprozesses des Halbzeugs ist die PVC-Folie für eine bestimmte Zeit einer Temperatur von $120{ }^{\circ} \mathrm{C}$ ausgesetzt. Da dieser thermische Einfluss bei der Produktion grundsätzlich vorhanden ist und einen Einfluss auf die Materialeigenschaften von PVC haben kann (vergl. Diemert et al. 2005), wurde neben der Zugfestigkeitsprüfung von zuvor im Normalklima gelagerter Proben eine weitere Versuchsreihe mit thermisch vorbehandelten Probekörpern durchgeführt. Hierzu wurde die Folie wie im üblichen Herstellungsprozess mit Furnier beleget, wobei kein Klebstoff zur Anwendung kam und bei $120^{\circ} \mathrm{C}$ für $120 \mathrm{~s}$ (zzgl. ca. $20 \mathrm{~s}$ für das Öffnen und Schließen der Presse) bei einem eingestellten Pressdruck von ca. $35 \mathrm{~N}^{*} \mathrm{~cm}^{-2}$ gepresst. Aufgrund eines langfristigen Defektes der Niederdruckstufe der Laborpresse musste auf die Hochdruckstufe gewechselt und die dort kleinstmögliche Einstellung gewählt werden. Daher weichen die Pressdrücke in diesem Kapitel zu denen aus Kapitel - $49-4.3$ ab.

Tabelle 4-2 zeigt die Ergebnisse des Zugfestigkeitsversuchs. Die Versuchsreihe, bei denen die Probekörper zuvor im Normalklima gelagert wurden, ist als Zugversuch „,norm“ tituliert. Die Versuchsreihe, bei der die Probekörper vor der Materialprüfung einer thermischen Behandlung ausgesetzt waren, wird als Zugversuch "temp“ bezeichnet.

Tabelle 4-2: Ergebnisse der Zugfestigkeitsprüfung der PVC-Folie in Anlieferungs- und temperiertem Zustand.

\begin{tabular}{|l|c|c|}
\hline Parameter & $\begin{array}{c}\text { Zugversuch "norm" } \\
\mathbf{n = 3 2} \\
{\left[\mathbf{N}^{*} \mathbf{m m}^{-2}\right]}\end{array}$ & $\begin{array}{c}\text { Zugversuch "temp" } \\
\mathbf{n}=\mathbf{3 5} \\
{\left[\mathbf{N}^{*} \mathbf{m m}^{-\mathbf{2}}\right]}\end{array}$ \\
\hline Median Z & 12,46 & 13,12 \\
\hline Arithmetisches Mittel $\boldsymbol{\mu}$ & 12,35 & 13,32 \\
\hline Standardabweichung $\mathbf{S}$ & 0,42 & 0,96 \\
\hline Minimum & 11,29 & 11,51 \\
\hline Maximum & 12,97 & 15,28 \\
\hline
\end{tabular}

Bei der Versuchsreihe „norm“ konnte eine mittlere Zugfestigkeit von 12,35 N*mm-2 bei einer Standardabweichung von $0,42 \mathrm{~N}^{*} \mathrm{~mm}^{-2}$ gemessen werden. Der mittlere Zugfestigkeitswert der Versuchsreihe "temp“ beläuft sich auf 13,32 $\mathrm{N}^{*} \mathrm{~mm}^{-2}$ $\left(\mathrm{S}=0,96 \mathrm{~N}^{*} \mathrm{~mm}^{-2}\right)$ und liegt somit $0,97 \mathrm{~N}^{*} \mathrm{~mm}^{-2}$ über dem Ergebnis der Versuchsreihe „norm“.

Die Extreme streuen bei „norm“ mit einem Minimum von 11,29 $\mathrm{N}^{*} \mathrm{~mm}^{-2}$ und einem Maximum von $12,97 \mathrm{~N}^{*} \mathrm{~mm}^{-2}$ um das arithmetische Mittel. 
In der Versuchsreihe "temp" wurde ein Minimum von $11,51 \mathrm{~N}^{*} \mathrm{~mm}^{-2}$ und ein Maximum von $15,28 \mathrm{~N}^{*} \mathrm{~mm}^{-2}$ gemessen.

Im Folgenden werden die Mittelwerte der beiden Versuchsreihen einander gegenüber gestellt, um zu untersuchen, ob ein signifikanter Unterschied zwischen den Grundgesamtheiten vorliegt. Die hierzu aufgestellten Hypothesen lauten:

$H_{0}$ : Die jeweiligen arithmetischen Mittelwerte der $F_{\max }$, bezogen auf die Zustände "normal“ und "thermisch behandelt", unterscheiden sich nicht.

H1: Die jeweiligen arithmetischen Mittelwerte der $F_{\max }$, bezogen auf die Zustände "normal" und "thermisch behandelt", unterscheiden sich signifikant.

Da die Untersuchung auf Normalverteilung innerhalb der Gruppen negativ verlief, erfolgte zum Vergleich der beiden Mittelwerte eine nichtparametrische Analyse. Als Testverfahren zum Vergleich der Mittelwerte wurde ein Kruskal-Wallis-Test durchgeführt.

Das Ergebnis der Analyse besagt, dass $\mathrm{H}_{0}$ verworfen und $\mathrm{H}_{1}$ angenommen werden muss. Somit unterscheiden sich die beiden Grundgesamtheiten bei einem Niveau von 0,05 signifikant voneinander.

\section{Diskussion der Versuchsergebnisse}

Wie der oben beschriebene Versuch zeigt, hat die während des Herstellungsprozesses des Halbzeugs auftretende Temperatur keinen "negativen“ Einfluss auf die Zugfestigkeit der PVC-Folie. Vielmehr führt der thermische Einfluss zu einer Erhöhung der Zugspannung. Ob die erhöhte Temperatur jedoch einen Einfluss auf andere Werkstoffeigenschaften hat, wurde nicht weitergehend untersucht.

Besonders hervorstechend ist die um 0,97 $\mathrm{N}^{*} \mathrm{~mm}^{-2}$ höhere Zugfestigkeit der Versuchsreihe "temp“ im Vergleich zur Versuchsreihe „norm“. Dies ist insofern auffällig, da Diemert et al. (2005) eine Abnahme der Zugspannung bei thermischer Belastung von PVC beschreiben. Auch Franck 2000 schildert ein Nachlassen der Zugspannung bei steigender thermischer Exposition von Thermoplasten. Im Unterschied $\mathrm{zu}$ den von Diemert et al. (2005) beschriebenen Kurven, die eine thermische Exposition des Kunststoffes ab einer Stunde und bei maximal $60{ }^{\circ} \mathrm{C}$ darstellen, betrug die thermische Exposition im vorliegenden Fall ca. $130 \mathrm{~s}$ bei $120^{\circ} \mathrm{C}$.

Die nachlassende Zugfestigkeit von thermoplastischen Kunststoffen bei erhöhter Temperatur ist sogar nötig, um diese verarbeiten zu können (vergl. Franck 2000). Da die Glasübergangstemperatur von weich-PVC (entspricht der hier verwendeten Folie) mit zunehmendem Weichmacheranteil deutlich sinkt (vergl. Diemert et al. 2005), könnte es bei der hier verwendeten Temperatur von $120^{\circ} \mathrm{C}$ und dem angewandten Pressdruck von $35 \mathrm{~N}^{*} \mathrm{~cm}^{-2}$ evtl. sein, dass es zu einer Neuausrichtung der Moleküle 
innerhalb der PVC-Folie kommt. Gepresste Kunststoffplatten verhalten sich im Gegensatz zu extrudierten Folien nahezu isotrop (Simona 2016).

Diese Hypothese, kann abschließend nicht geklärt werden.

Betrachtet man jedoch hierzu Abbildung 4-3 und vergleicht Versuchsreihen „norm“ und "temp“, könnten die gemessenen Unterschiede der Zugfestigkeit evtl. auf einen Messfehler zurückzuführen sein. Da der Stichprobenumfang bei den Mittelwertvergleichen in Kapitel 4.2.2 nur $n=32$ bzw. $n=35$ beträgt und die Standardabweichung bei der Versuchsreihe "temp“ deutlich höher ausfällt als bei „,norm“, muss hier davon ausgegangen werden, dass bei höherem Stichprobenumfang kein signifikanter Unterschied zwischen den Mittelwerten zu erkennen sein würde.

Als Fazit lässt sich jedoch festhalten, dass Bedenken hinsichtlich des Herstellungsprozesses und der gewählten Prozesstemperatur von $120^{\circ} \mathrm{C}$ bezogen auf die PVC-Folie, negiert werden können.

\subsubsection{Untersuchung der Zugfestigkeit in verschiedenen Winkeln}

Wie in Kapitel 3.2.2.7 beschrieben, erfolgte in Anlehnung an DIN ISO 527 die Messung der Zugfestigkeit der Folie in verschiedenen Einspannwinkeln. Entgegen der Zugfestigkeitsprüfung in Kapitel 4.2.2 wurde die Folie im gesamten Werkstoffverbund überprüft, da dies den Bedingungen im realen „Einsatz“ am nächsten kommt. Zudem dienen die Decklagen dazu, die Probekörper im exakten Winkel einspannen zu können, was ohne diese nur schwer zu realisieren ist.

Tabelle 4-3: Statistische Ergebnisse der Zugversuche an PVC-Folie im angewinkelten Zustand.

\begin{tabular}{|c|c|c|c|c|c|c|}
\hline Parameter & $\begin{array}{c}\text { Zugversuch } \\
\text { "450" } \\
\text { "parallel" } \\
n=40 \\
{\left[\mathrm{~N}^{*} \mathrm{~mm}^{-2}\right]}\end{array}$ & $\begin{array}{c}\text { Zugversuch } \\
\text { "45 } \\
\text { "senkrecht" } \\
\mathrm{n}=39 \\
{\left[\mathrm{~N}^{*} \mathrm{~mm}^{-2}\right]}\end{array}$ & $\begin{array}{c}\text { Zugversuch } \\
\text { "450" } \\
\text { "gesamt" } \\
\text { n }=79 \\
{\left[\mathrm{~N}^{*} \mathrm{~mm}^{-2}\right]}\end{array}$ & $\begin{array}{c}\text { Zugversuch } \\
\text { "90 } \\
\text { "parallel" } \\
\text { n }=42 \\
{\left[\mathrm{~N}^{*} \mathrm{~mm}^{-2}\right]}\end{array}$ & $\begin{array}{c}\text { Zugversuch } \\
\text { "90 } \\
\text { "senkrecht" } \\
n=40 \\
{\left[N^{*} \mathrm{~mm}^{-2}\right]}\end{array}$ & $\begin{array}{c}\text { Zugversuch } \\
\text { "90" } \\
\text { "gesamt" } \\
\text { n }=82 \\
{\left[\mathrm{~N}^{*} \mathrm{~mm}^{-2}\right]}\end{array}$ \\
\hline Median Z & 11,93 & 10,31 & 10,53 & 12,04 & 11,85 & 11,90 \\
\hline $\begin{array}{l}\text { Arithmetisches } \\
\text { Mittel } \mu\end{array}$ & 11,59 & 10,30 & 10,95 & 11,94 & 11,79 & 11,87 \\
\hline $\begin{array}{l}\text { Standardab- } \\
\text { weichung S }\end{array}$ & 1,35 & 0,54 & 1,21 & 0,85 & 1,05 & 0,95 \\
\hline Minimum & 9,29 & 9,06 & 9,06 & 10,61 & 9,77 & 9,77 \\
\hline Maximum & 14,19 & 11,36 & 14,19 & 13,87 & 14,41 & 14,41 \\
\hline
\end{tabular}

Wie aus Tabelle 4-3 ersichtlich, gibt es innerhalb der Zugversuchsreihen als auch zwischen den Zugversuchen „ $45^{\circ}$ “ und „90“ differierende Festigkeitswerte.

\section{Versuchsreihe mit Einspannwinkel „45 $45^{\circ}$}

In der Versuchsreihe ", $45^{\circ}$ (parallel zur Extrusionsrichtung) wurde eine mittlere Zugfestigkeit von 11,59 $\mathrm{N}^{*} \mathrm{~mm}^{-2}\left(\mathrm{~S}=1,35 \mathrm{~N}^{*} \mathrm{~mm}^{-2}\right)$ gemessen. Die mittlere Zugfestigkeit 
bei ", $45^{\circ}$ “ (senkrecht zur Extrusionsrichtung) belief sich hingegen auf $10,30 \mathrm{~N}^{*} \mathrm{~mm}^{-2}$ $\left(\mathrm{S}=0,54 \mathrm{~N}^{*} \mathrm{~mm}^{-2}\right)$.

Die Differenz beträgt somit innerhalb der Messreihe „ $45^{\circ}$ “ $1,29 \mathrm{~N}^{*} \mathrm{~mm}^{-2}$ zwischen den Lagen „parallel“ und „senkrecht". Somit lauten die Hypothesen:

$\mathrm{H}_{0}$ : Die jeweiligen arithmetischen Mittelwerte der Zugspannung beim modifizierten Prüfwinkel von $45^{\circ}$, bezogen auf die Extrusionsrichtung "parallel“ und "senkrecht", unterscheiden sich nicht.

H1: Die jeweiligen arithmetischen Mittelwerte der Zugspannung bei einem modifizierten Prüfwinkel von $45^{\circ}$, bezogen auf die Extrusionsrichtung "parallel“ und ",senkrecht", unterscheiden sich signifikant.

Da keine Normalverteilung der Messwerte innerhalb Datenreihe „parallel“ gegeben war, wurde zum Vergleich der Mittelwerte zwischen den Messreihen ein KruskalWallis-Test durchgeführt. Dieser ergab bei einem Niveau von 0,05 einen signifikanten Unterschied der beiden Mittelwerte. Somit muss $\mathrm{H}_{1}$ angenommen und $\mathrm{H}_{0}$ verworfen werden.

\section{Versuchsreihe mit Einspannwinkel „90\%}

Wie Tabelle 4-3 zeigt, konnte bei der Versuchsreihe "90 " (parallel zur Extrusionsrichtung) eine mittlere Zugfestigkeit von $11,94 \mathrm{~N}^{*} \mathrm{~mm}^{-2}\left(\mathrm{~S}=0,85 \mathrm{~N}^{*} \mathrm{~mm}^{-2}\right)$ ermittelt werden. Der Mittelwert der Zugversuche von "90 “ (senkrecht zur Extrusionsrichtung) wurde mit $, 11,79 \mathrm{~N}^{*} \mathrm{~mm}^{-2} \quad\left(\mathrm{~S}=1,05 \mathrm{~N}^{*} \mathrm{~mm}^{-2}\right)$ bestimmt. Dementsprechend liegt innerhalb der Versuchsreihe ",90“" eine Differenz zwischen den Lagen „parallel“ und „senkrecht“ in Höhe von 0,15 N*mm² vor.

Aus diesem Grund werden folgen Hypothesen aufgestellt:

$\mathrm{H}_{0}$ : Die jeweiligen arithmetischen Mittelwerte der Zugspannung beim modifizierten Prüfwinkel von $90^{\circ}$, bezogen auf die Extrusionsrichtung "parallel“ und "senkrecht" unterscheiden sich nicht.

$H_{1}$ : Die jeweiligen arithmetischen Mittelwerte der Zugspannung bei einem modifizierten Prüfwinkel von $90^{\circ}$, bezogen auf die Extrusionsrichtung "parallel“ und "senkrecht" unterscheiden sich signifikant.

Da im Falle der Datenreihe „senkrecht" eine nicht normalverteilte Grundgesamtheit vorliegt, wurde der Vergleich der beiden Mittelwerte mittels Kruskal-Wallis-Tests durchgeführt. Das Ergebnis besagt, dass sich bei einem Niveau von 0,05 die beiden Grundgesamtheiten signifikant unterscheiden. Somit muss $\mathrm{H}_{0}$ verworfen und $\mathrm{H}_{1}$ angenommen werden.

Vergleichende Diskussion der Versuchsergebnisse ${ }^{4} 45^{\circ}$ und ${ }^{\circ} 90^{\circ}$ 
Die Versuchsreihen " $45^{\circ}$ " und "9 $90^{\circ}$ " weisen eine Differenz in ihrer Zugfestigkeit von $0,92 \mathrm{~N}^{*} \mathrm{~mm}^{-2}$ auf, wobei die Versuchsreihe "90 " 9 mit $\overline{\mathrm{x}}=11,87 \mathrm{~N}^{*} \mathrm{~mm}^{-2}$ die höheren Festigkeitswerte im Vergleich zur Versuchsreihe „, $45^{\circ}$ “ mit $\bar{x}=10,95 \mathrm{~N}^{*} \mathrm{~mm}^{-2}$ erreicht.

Zugleich fällt in beiden Messreihen auf, dass die Zugfestigkeiten parallel zur Extrusionsrichtung signifikant höher ausfallen als senkrecht zur Extrusionsrichtung. Dies entspricht den Ergebnissen aus Kapitel 4.2.1 und zeigt, dass die Orientierungsrichtung in der PVC-Folie, die während des Extrusionsvorgangs zustande kommt, auch hier den wie von Simona (2016) beschrieben Einfluss auf die Festigkeit hat.

Beim Vergleich zwischen der Versuchsreihe nach DIN-EN ISO 527-3 mit den beiden modifizierten Messreihen ergibt sich jedoch ein anderes Bild wie Tabelle 4-4 zeigt.

Tabelle 4-4: Vergleichende Darstellung der Messwerte der Versuchsreihen "norm", "45" und "90".

\begin{tabular}{|c|c|c|c|}
\hline Parameter & $\begin{array}{c}\text { Zugversuch "norm" } \\
\text { "gesamt" } \\
\mathbf{n}=78 \\
{\left[\mathbf{N}^{*} \mathrm{~mm}^{-2}\right]}\end{array}$ & 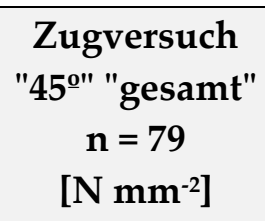 & $\begin{array}{c}\text { Zugversuch } \\
\text { "90 } 0^{\mathrm{o} " ~ " g e s a m t "} \\
\mathbf{n}=82 \\
{\left[\mathrm{~N} \mathrm{~mm}^{-2}\right]}\end{array}$ \\
\hline Median Z & 12,90 & 10,53 & 11,90 \\
\hline Arithmetisches Mittel $\mu$ & 12,96 & 10,95 & 11,87 \\
\hline Standardabweichung S & 1,26 & 1,21 & 0,95 \\
\hline Minimum & 11,21 & 9,06 & 9,77 \\
\hline Maximum & 14,69 & 14,19 & 14,41 \\
\hline
\end{tabular}

Beim Zugversuch „,norm“ wurde eine Festigkeit von $\bar{x}=12,96 \mathrm{~N}^{*} \mathrm{~mm}^{-2}\left(\mathrm{~S}=1,26 \mathrm{~N}^{*} \mathrm{~mm}^{-2}\right)$, bei " $45^{\circ}$ “ eine Zugfestigkeit von $\bar{x}=10,95 \mathrm{~N}^{*} \mathrm{~mm}^{-2}\left(\mathrm{~S}=1,21 \mathrm{~N}^{*} \mathrm{~mm}^{-2}\right)$ und bei "90" eine solche von $\bar{x}=11,87 \mathrm{~N}^{*} \mathrm{~mm}^{-2}\left(\mathrm{~S}=0,95 \mathrm{~N}^{*} \mathrm{~mm}^{-2}\right)$ erreicht.

Auf Grundlage dieser Ergebnisse wurden folgende Hypothesen aufgestellt:

$\mathrm{H}_{0}$ : Die jeweiligen arithmetischen Mittelwerte der Zugspannungen der einzelnen Versuchsreihen unterscheiden sich nicht.

$H_{1}$ : Die jeweiligen arithmetischen Mittelwerte der Zugspannungen der einzelnen Versuchsreihen unterscheiden sich signifikant.

Die Überprüfung der Mittelwerte mittels Kruskal-Wallis-Tests ergab, dass sich die drei Grundgesamtheiten bei einem Niveau von 0,05 signifikant unterscheiden.

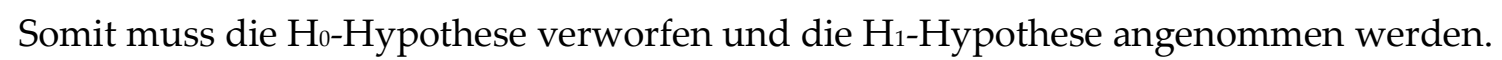




\section{Vergleichende Diskussion der Versuchsergebnisse "norm "}

Bereits vor Versuchsbeginn wurde vermutet, dass die Festigkeitswerte der Versuchsreihen " $45^{\circ}$ “ und "90" geringer ausfallen als bei der Versuchsreihe "norm", da von einem Einfluss der Furnierkante auf den Kunststoff auszugehen ist. Wie aus Tabelle 4-4 und Abbildung 4-3 ersichtlich, bestätigte sich diese Annahme. Im Vergleich zur Versuchsreihe "90“ liegt das arithmetische Mittel der Zugfestigkeit im Zugversuch „norm“ um 1,09 $\mathrm{N}^{*} \mathrm{~mm}^{-2}$ und somit um 9,18\% höher. Im Falle der Versuchsreihe „ $45^{\circ}$ “ beträgt die Differenz des arithmetischen Mittels zur Reihe „norm“ sogar $18,36 \%$.

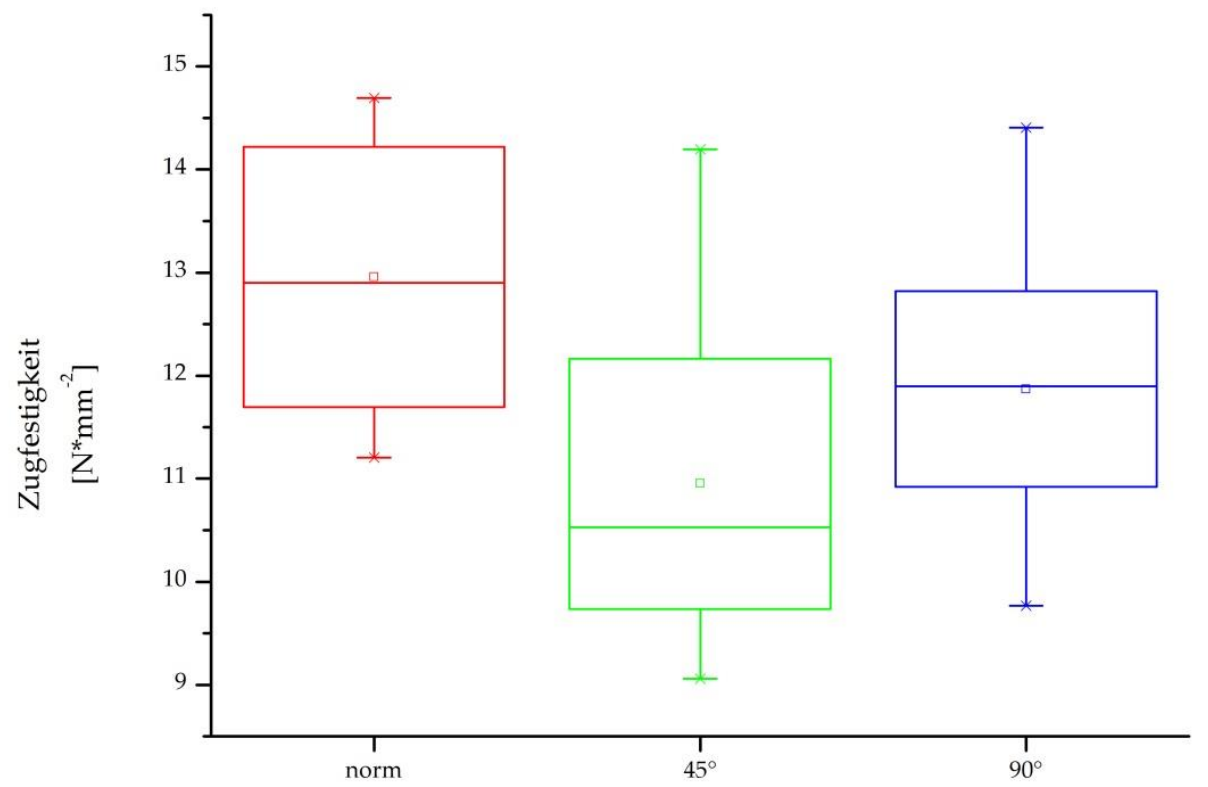

Abbildung 4-3: Boxplots der ermittelten Zugfestigkeiten zu den Messreihen „norm“, „45“" und „90".

Da die statistischen Untersuchungen die Signifikanz der Unterschiede der Mittelwerte zwischen den einzelnen Versuchsreihen bestätigen, ist von einem Einfluss zu tief gefräster Faltnuten und/oder asymmetrische gefräster Nuten auszugehen (vergl. Abbildung 4-4). Den entscheidenderen Einfluss dürften zu tief gefräste Nuten haben, da diese zu einer Reduzierung des Querschnitts der Mittelschicht führen (siehe auch Werndl 2016). 


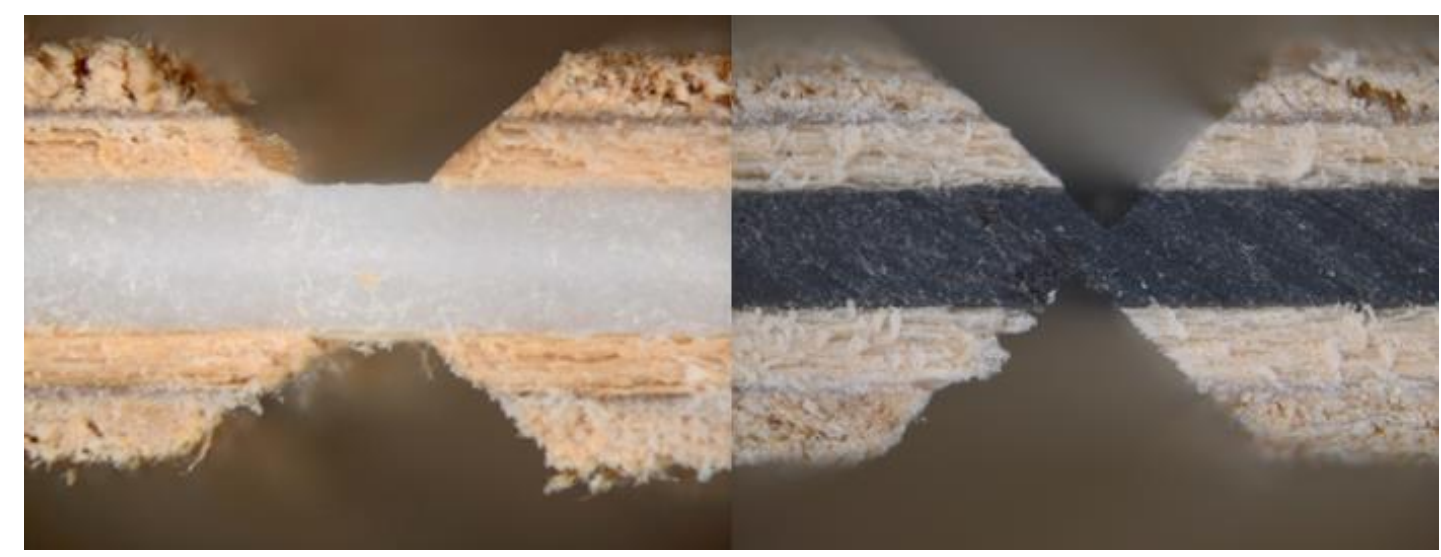

Abbildung 4-4: Ideales Fräsbild (links), fehlerhafte Fräsnuten mit Versatz und zu hoher Einritztiefe (rechts) (Foto: P. Werndl).

Bei einem starken Versatz der Fräsnuten, wie z. B. in Abbildung 4-5 ersichtlich, kann sich die Decklage aus Furnier, bei angewinkelter Materialprüfung über die Versatzkante ähnlich einer Klinge auf die Mittellage der Kunststoffschicht auswirken und somit zu verringerten Festigkeitswerten führen.

Weiterhin sollte jedoch auch die Möglichkeit in Betracht gezogen werden, dass die durch den Anstellwinkel entstehende Vorspannung auf der Außenseite des Prüfkörpers bzw. die Stauchung auf der Innenseite zu einem Festigkeitsverlust führen kann, da sich die Belastungen auf die Mittelschicht deutlich von der im normativen Zugversuch unterscheiden (Werndl 2016).

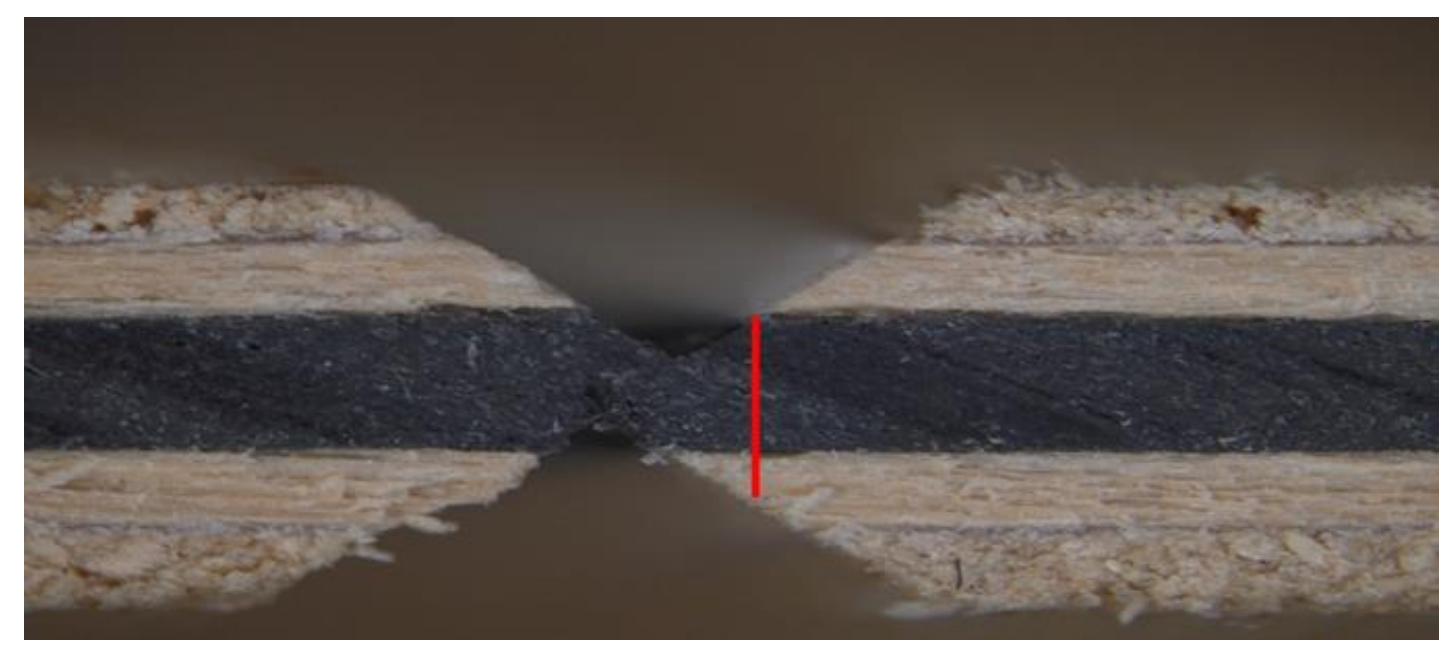

Abbildung 4-5: Stark asymmetrischer Versatz der Fräsnuten. (Foto: P. Werndl)

Daher ist bei der Herstellung von Produkten aus Faltfurnier ein besonders hohes Augenmerk auf die Einhaltung exakter Fräsvorgänge zu legen, um negative Einflüsse auf die Festigkeitseigenschaften des Endproduktes zu vermeiden. 


\subsection{Untersuchung von Abhebefestigkeiten verschiedener Werkstoffverbünde auf der Basis von Erlen- und Eichenfurnieren}

In den folgenden Kapiteln werden zunächst die adhäsiven Eigenschaften und die Qualität der Verklebungen von Erlenfurnier auf PVC-Folie (mittels thermoplastischem Klebefilm) bei verschiedenen Presszeiten untersucht. Diese Versuchsriehen dienen der Bestimmung der optimalen Presszeit für den Verbundwerkstoff unter der Berücksichtigung von hoher Festigkeit bei möglichst kurzer Presszeit (vergl. Kapitel 1.2. und 4.1).

Im Anschluss erfolgt eine analoge Versuchsreihe mit Eichenfurnieren anstelle von Erlenfurnier.

Zudem sollen die zwei verwendeten Klebstoffsysteme, PVAc-Leim und PU-basierter thermoplastischer Klebefilm, auf ihre Eignung zur Verklebung von Unter- und Deckfurnier mittels Abhebefestigkeitsprüfung untersucht werden. Für den Vergleich wird Erlenfurnier mit Erlenfurnier verklebt.

\subsubsection{Untersuchung der Abhebefestigkeit von Erlenfurnier auf PVC-Folie}

Die Bestimmung der Abhebefestigkeit der Oberfläche nach DIN EN 311 gilt u. a. für beschichtete Holzwerkstoffe. Ziel ist die Ermittlung der Festigkeit bzw. Qualität der adhäsiven Eigenschaften, im Falle von beschichteten Platten, zwischen der Beschichtung und der darunter liegenden Platte. Hierbei wird die Zugkraft festgestellt, die nötig ist, um eine bestimmte Fläche von einer Oberfläche abzuziehen (Deutsche Norm DIN EN 311).

Im vorliegenden Fall wurden die Festigkeitseigenschaft, also die Bruchkraft bestimmt, die nötig ist, um das Furnier von der PVC-Folie zu lösen.

Ziel war die Ermittlung der höchsten Bruchkraft, bei gleichzeitig möglichst kurzer Pressdauer. Die Untersuchung erfolgte an Probekörpern, die von Platten stammten, die bei verschiedenen Presszeiten nämlich 60 s, 90 s, 120 s, 180 s und 240 s gepresst wurden. Als minimales Ziel galt eine Bruchkraft von $0,8 \mathrm{~N}^{*} \mathrm{~mm}^{-2}$. Dies entspricht der Mindestanforderung an Spanplatten (Niemz und Sonderegger 2017).

Tabelle 4-5 zeigt die Herstellungsparameter der untersuchten Platten. 
Das Plattenformat hatte einheitlich die Maße $750 \mathrm{~mm}$ x $300 \mathrm{~mm}$. Die PVC-Folie wurde beidseitig mittels des Klebstoffs Collano 46.002 / 100 g*m$^{-2}$ (vergl. Kapitel 3.2.3.2) mit jeweils einem Furnierblatt aus Erle beklebt. Die gemittelten Furnierdicken lagen bei 0,54 $\mathrm{mm}$ und die Foliendicke bei 0,91 $\mathrm{mm}$.

Da produktionsbedingt minimale Schwankungen sowohl zwischen den Einzelwerten der Furnierblätter, als auch der PVC-Folie gegeben sind, wurden die stichprobeartig genommenen Messwerte arithmetisch gemittelt.

Tabelle 4-5: Herstellungsparameter des untersuchten Materialverbunds aus Erle auf PVC.

\begin{tabular}{|l|l|}
\hline Material & $\begin{array}{l}\text { Messerfurniere Erle } \\
\text { PVC-Folie }\end{array}$ \\
\hline Plattenformat & $750 \mathrm{~mm} \times 300 \mathrm{~mm}$ \\
\hline Furnierdicke & $\overline{\mathrm{x}}=0,54 \mathrm{~mm}$ \\
\hline Foliendicke & $\overline{\mathrm{x}}=0,91 \mathrm{~mm}$ \\
\hline Klebstoff MS-Absperrfurnier & Collano $46.002 / 100 \mathrm{~g}^{*} \mathrm{~m}^{-2}$ \\
\hline Presstemperatur & $120^{\circ} \mathrm{C}$ \\
\hline Pressdruck & $13,96 \mathrm{~N}^{*} \mathrm{~cm}^{-2}$ \\
\hline Schließzeit Presse & $\overline{\mathrm{x}}=8,83 \mathrm{~s}$ \\
\hline
\end{tabular}

Der Pressdruck ergibt sich aus dem an der Presse eingestellten Druck von 10 bar (entspricht $100 \mathrm{~N}^{*} \mathrm{~cm}^{-2}$ ), der Kolbenfläche der Presse von $314 \mathrm{~cm}^{2}$ sowie der Fläche des Verbundmaterials von $2.250 \mathrm{~cm}^{2}$.

Die Begründung zur Auswahl der Parameter Pressdruck und Temperatur findet sich in Kapitel 4.1.

Die im Folgenden verwendeten Namen der Versuchsreihen „oben“, „unten“, „Mitte“ und "Rand" bezeichnen ihre Entnahmestelle aus den Probeplatten; also Oberseite, Unterseite, Mittel- und Randlage. Als "gesamt“ werden sämtliche zu einer Presszeit gehörigen Messwerte, lageunabhängig, tituliert.

Die Differenzen des Umfangs der Grundgesamtheiten der folgenden Versuchsreihen stammen aus fehlerhaften Messungen bzw. Probekörpern (vergl. Abbildung 4-6.) 


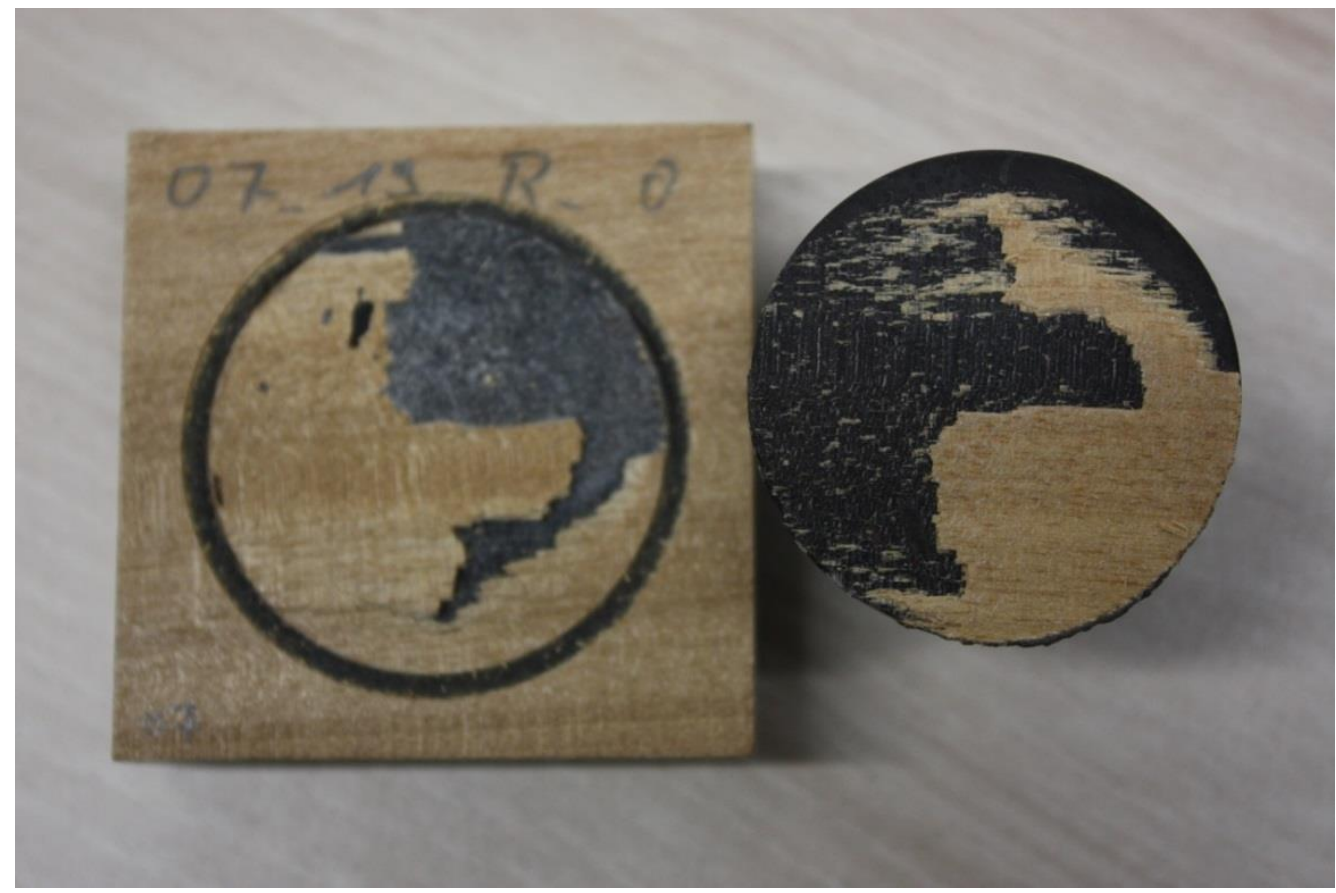

Abbildung 4-6: Beispiel für eine fehlerhafte Abhebefestigkeitsprüfung. Das Furnier wurde durch den Prüfpilz nicht flächig abgelöst und das Versuchsergebnis somit verworfen.

\section{Presszeit 60 Sekunden}

Bei einer Presszeit von 60 s wurde wie Tabelle 4-6 darstellt, eine maximale Bruchkraft (Fmax) von $\bar{x}=1,25 \mathrm{~N}^{*} \mathrm{~mm}^{-2}$ bei einer Standardabweichung von $0,31 \mathrm{~N}^{*} \mathrm{~mm}^{-2}$ erreicht. Wie weiterhin aus dieser Tabelle ersichtlich, differieren die arithmetischen Mittel der Fmax zwischen den einzelnen Lagen in den Probeplatten um maximal 0,05 $\mathrm{N}^{*} \mathrm{~mm}^{-2}$. Dies entspricht, bezogen auf den höchsten Mittelwert von 1,27 $\mathrm{N}^{*} \mathrm{~mm}^{-2}$, bei Lage der Probeköper auf der unteren Plattenseite, einer Abweichung von 3,94 \%.

Deutlich höher differieren hingegen die Extremwerte. So liegt der Minimalwert bei lediglich $0,46 \mathrm{~N}^{*} \mathrm{~mm}^{-2}$ und der Maximalwert bei $1,84 \mathrm{~N}^{*} \mathrm{~mm}^{-2}$. Dies bedeutet eine Differenz von $1,38 \mathrm{~N}^{*} \mathrm{~mm}^{-2}$. 
Tabelle 4-6: Ergebnisse der Abhebefestigkeitsprüfung von Erlenfurnier auf PVC bei 60 Sekunden Presszeit, differenziert nach dem Gesamtergebnis und den jeweiligen Lagen der Probekörper in den Probeplatten.

\begin{tabular}{|l|c|c|c|c|c|}
\hline Parameter & $\begin{array}{c}\text { Abhebefestig- } \\
\text { keit } \\
\text { "gesamt" } \\
\mathbf{n}=57 \\
{\left[\mathbf{N}^{*} \mathbf{m m}-\mathbf{2}\right]}\end{array}$ & $\begin{array}{c}\text { Abhebefestig- } \\
\text { keit } \\
\text { "oben" } \\
\mathbf{n = 2 6} \\
{\left[\mathbf{N}^{*} \mathbf{m m}^{-2}\right]}\end{array}$ & $\begin{array}{c}\text { Abhebefestig- } \\
\text { keit } \\
\text { "unten" } \\
\mathbf{n = 3 1} \\
{\left[\mathbf{N}^{*} \mathbf{m m}^{-2}\right]}\end{array}$ & $\begin{array}{c}\text { Abhebefestig- } \\
\text { keit } \\
\text { "Mitte" } \\
\mathbf{n}=57 \\
{\left[\mathbf{N}^{*} \mathbf{m m} \mathbf{m}^{-2}\right]}\end{array}$ & $\begin{array}{c}\text { Abhebefestig- } \\
\text { keit } \\
\text { "Rand" } \\
\mathbf{n}=\mathbf{2 5} \\
{\left[\mathbf{N}^{*} \mathbf{m m}^{-2}\right]}\end{array}$ \\
\hline Presszeit s & 60 & 60 & 60 & 60 & 60 \\
\hline Median Z & 1,24 & 1,21 & 1,29 & 1,23 & 1,25 \\
\hline $\begin{array}{l}\text { Arithmetisches } \\
\text { Mittel } \boldsymbol{\mu}\end{array}$ & 1,25 & 1,22 & 1,27 & 1,25 & 1,24 \\
\hline $\begin{array}{l}\text { Standardab- } \\
\text { weichung S }\end{array}$ & 0,31 & 0,29 & 0,34 & 0,27 & 0,37 \\
\hline Minimum & 0,46 & 0,46 & 0,48 & 0,54 & 0,46 \\
\hline Maximum & 1,84 & 1,68 & 1,84 & 1,72 & 1,84 \\
\hline
\end{tabular}

Die beiden aufzustellenden Hypothesen lauten:

$H_{0}$ : Die jeweiligen arithmetischen Mittelwerte der $F_{\max }$, bezogen auf die Lage der Probekörper in der Prüfplatte, unterscheiden sich nicht.

$H_{1}$ : Die jeweiligen arithmetischen Mittelwerte der $F_{\max }$, bezogen auf die Lage der Probekörper in der Prüfplatte, unterscheiden sich signifikant.

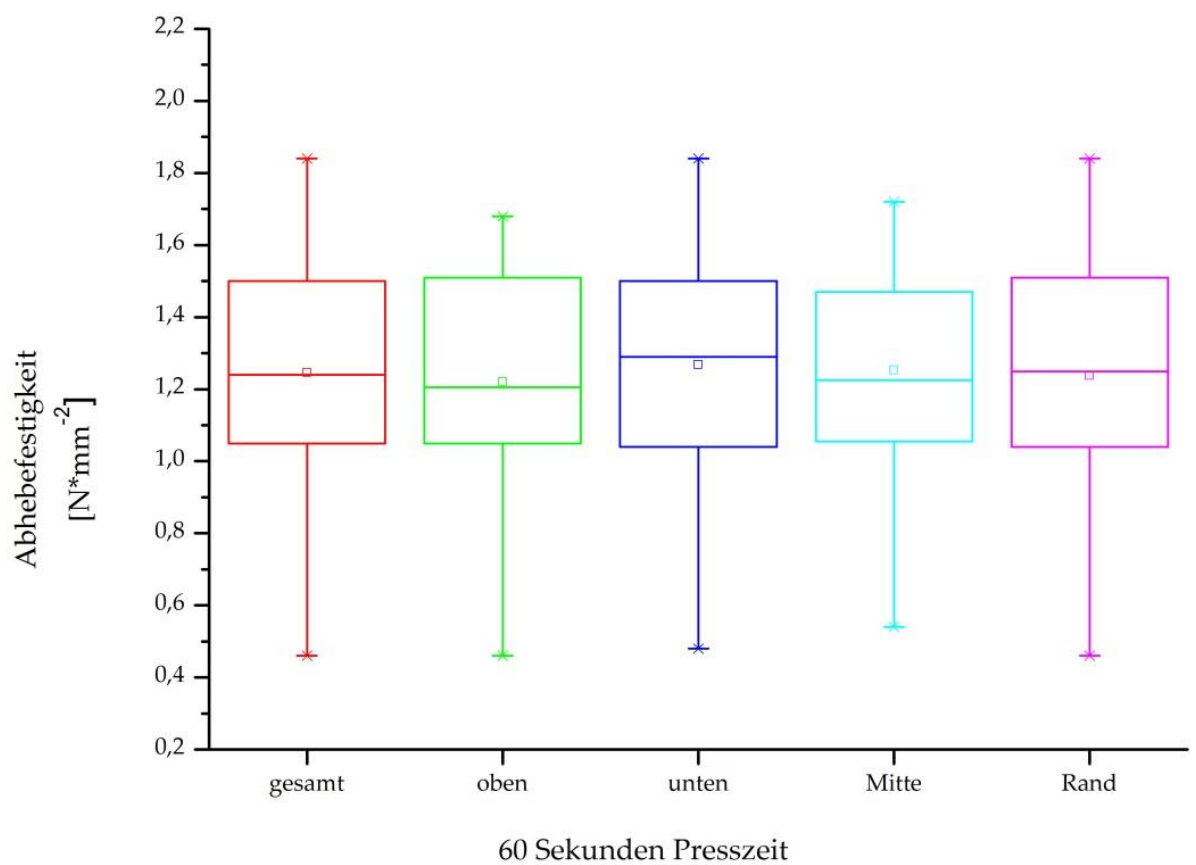

Abbildung 4-7: Boxplots zur Abhebefestigkeit von Erlenfurnier auf PVC bei 60 Sekunden Presszeit, sortiert nach Gesamtergebnis sowie der Probenlage innerhalb der Platten. 
Sieht man von den Extremwerten ab und betrachtet die in Abbildung 4-7 dargestellten Lage- und Streuparameter sowie die errechneten Standardabweichungen ist davon auszugehen, dass sich die Fmax zwischen den einzelnen Lagen in den Probeplatten nicht signifikant voneinander unterscheiden.

Die statistische Auswertung mittels ANOVA bestätigt diese Annahme. Bei einem Signifikanzniveau von 0,05 unterscheiden sich die Mittelwerte der Grundgesamtheiten nicht signifikant voneinander. Somit muss $\mathrm{H}_{0}$ angenommen und $\mathrm{H}_{1}$ verworfen werden.

\section{Presszeit 90 Sekunden}

Bei einer Presszeit von $90 \mathrm{~s}$ wurde, wie in Tabelle 4-7 dargestellt, eine maximale Bruchkraft (Fmax) von $\bar{x}=1,17 \mathrm{~N}^{*} \mathrm{~mm}^{-2}$ bei einer Standardabweichung von $0,28 \mathrm{~N}^{*} \mathrm{~mm}^{-2}$ erreicht. Wie weiterhin aus dieser Tabelle ersichtlich, differieren die Mittelwerte der Fmax zwischen den einzelnen Lagen in den Probeplatten um maximal 0,08 $\mathrm{N}^{*} \mathrm{~mm}^{-2}$, was einer Abweichung von 6,61\% bezogen auf den höchsten Mittelwert von $1,21 \mathrm{~N}^{*} \mathrm{~mm}^{-2}$ entspricht. Die Extrema liegen bei $0,58 \mathrm{~N}^{*} \mathrm{~mm}^{-2}$ bzw. $1,82 \mathrm{~N}^{*} \mathrm{~mm}^{-2}$ : Die Differenz entspricht somit $1,24 \mathrm{~N}^{*} \mathrm{~mm}^{-2}$.

Tabelle 4-7: Ergebnisse der Abhebefestigkeitsprüfung von Erlenfurnier auf PVC bei 90 Sekunden Presszeit, differenziert nach dem Gesamtergebnis und den jeweiligen Lagen der Probekörper in den Probeplatten.

\begin{tabular}{|l|c|c|c|c|c|}
\hline Parameter & $\begin{array}{c}\text { Abhebefestig- } \\
\text { keit } \\
\text { "gesamt" } \\
\mathbf{n = 6 1} \\
{\left[\mathbf{N}^{*} \mathbf{m m}^{-2}\right]}\end{array}$ & $\begin{array}{c}\text { Abhebefestig- } \\
\text { keit } \\
\text { "oben" } \\
\mathbf{n = 2 9} \\
{\left[\mathbf{N}^{*} \mathbf{m m}^{-2}\right]}\end{array}$ & $\begin{array}{c}\text { Abhebefestig- } \\
\text { keit } \\
\text { "unten" } \\
\mathbf{n = 3 2} \\
{\left[\mathbf{N}^{*} \mathbf{m m}^{-2}\right]}\end{array}$ & $\begin{array}{c}\text { Abhebefestig- } \\
\text { keit } \\
\text { "Mitte" } \\
\mathbf{n = 3 2} \\
{\left[\mathbf{N}^{*} \mathbf{m m}^{-2}\right]}\end{array}$ & $\begin{array}{c}\text { Abhebefestig- } \\
\text { keit } \\
\text { "Rand" } \\
\mathbf{n = 2 9} \\
{\left[\mathbf{N}^{*} \mathbf{m m}^{-2}\right]}\end{array}$ \\
\hline Presszeit s & 90 & 90 & 90 & 90 & 90 \\
\hline Median Z & 1,18 & 1,11 & 1,20 & 1,17 & 1,19 \\
\hline $\begin{array}{l}\text { Arithmetisches } \\
\text { Mittel } \boldsymbol{\mu}\end{array}$ & 1,17 & 1,16 & 1,19 & 1,13 & 1,21 \\
\hline $\begin{array}{l}\text { Standardab- } \\
\text { weichung S }\end{array}$ & 0,28 & 0,29 & 0,27 & 0,24 & 0,31 \\
\hline Minimum & 0,58 & 0,60 & 0,58 & 0,58 & 0,73 \\
\hline Maximum & 1,82 & 1,70 & 1,82 & 1,68 & 1,82 \\
\hline
\end{tabular}

Die beiden aufzustellenden Hypothesen lauten:

$H_{0}$ : Die jeweiligen arithmetischen Mittelwerte der $F_{\max }$, bezogen auf die Lage der Probekörper in der Prüfplatte, unterscheiden sich nicht.

$H_{1}$ : Die jeweiligen arithmetischen Mittelwerte der $F_{\max }$, bezogen auf die Lage der Probekörper in der Prüfplatte, unterscheiden sich signifikant.

Trotz der hohen Differenz zwischen den beiden Extremwerten lassen die Standardabweichung, wie auch grafisch in Abbildung 4-8 dargestellt, darauf schließen, dass sich die Mittelwerte der Fmax zwischen den einzelnen Lagen in den Probeplatten nicht signifikant voneinander unterscheiden. 


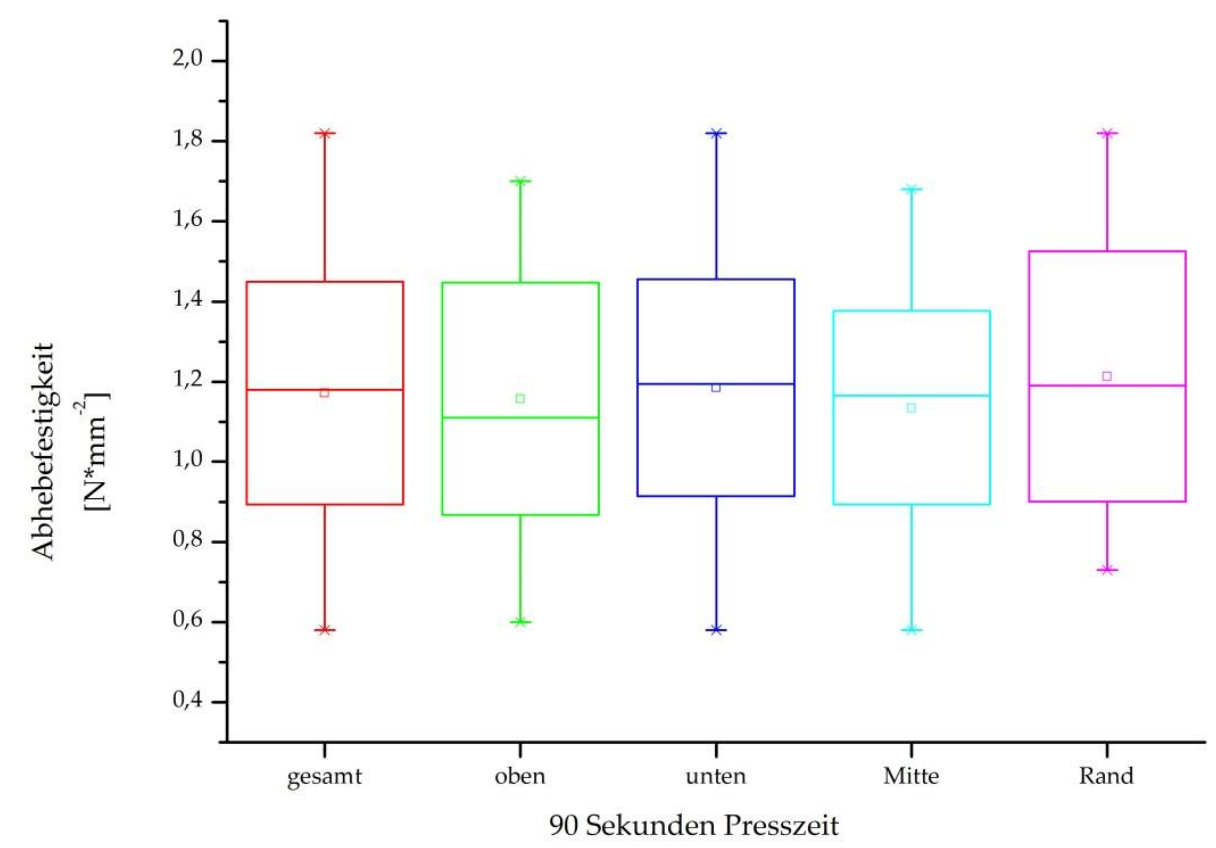

Abbildung 4-8: Boxplots zur Abhebefestigkeit von Erlenfurnier auf PVC bei 90 Sekunden Presszeit, sortiert nach Gesamtergebnis sowie der Probenlage innerhalb der Platten.

Die statistische Auswertung mittels ANOVA bestätigt diese Annahme. Bei einem Signifikanzniveau von 0,05 unterscheiden sich die Mittelwerte der Grundgesamtheiten nicht signifikant voneinander. Somit muss $\mathrm{H}_{0}$ angenommen und $\mathrm{H}_{1}$ verworfen werden.

\section{Presszeit 120 Sekunden}

Bei einer Presszeit von $120 \mathrm{~s}$ wurde wie aus Tabelle 4-8 ersichtlich, eine im Mittel maximale Bruchkraft (Fmax) von $\bar{x}=1,71 \mathrm{~N}^{*} \mathrm{~mm}^{-2}$, bei einer Standardabweichung von $0,27 \mathrm{~N}^{*} \mathrm{~mm}^{-2}$, erreicht. Die Extrema liegen wie auch bei den vorangegangenen Versuchsreihen „60 s" und „90 s" mit 1,11 N* $\mathrm{mm}^{-2}$ deutlich auseinander. Wie weiterhin aus dieser Tabelle ersichtlich, unterscheiden sich die Werte der Fmax zwischen den einzelnen Lagen in den Probeplatten um maximal 5,17 \% bezogen auf den höchsten Mittelwert von 1,75 N*mm-2 im Lagebereich „Rand“.

Die beiden aufzustellenden Hypothesen lauten:

$H_{0}$ : Die jeweiligen arithmetischen Mittelwerte der $F_{\max }$, bezogen auf die Lage der Probekörper in der Prüfplatte, unterscheiden sich nicht.

$H_{1}$ : Die jeweiligen arithmetischen Mittelwerte der $F_{\max }$, bezogen auf die Lage der Probekörper in der Prüfplatte, unterscheiden sich signifikant. 
Tabelle 4-8: Ergebnisse der Abhebefestigkeitsprüfung von Erlenfurnier auf PVC bei 120 Sekunden Presszeit, differenziert nach dem Gesamtergebnis und den jeweiligen Lagen der Probekörper in den Probeplatten.

\begin{tabular}{|l|c|c|c|c|c|}
\hline Parameter & $\begin{array}{c}\text { Abhebefestig- } \\
\text { keit } \\
\text { "gesamt" } \\
\mathbf{n = 6 8} \\
{\left[\mathbf{N}^{*} \mathbf{m m}^{-2}\right]}\end{array}$ & $\begin{array}{c}\text { Abhebefestig- } \\
\text { keit } \\
\text { oben" } \\
\mathbf{n = 3 4} \\
{\left[\mathbf{N}^{*} \mathbf{m m}^{-2}\right]}\end{array}$ & $\begin{array}{c}\text { Abhebefestig- } \\
\text { keit } \\
\text { unten" } \\
\mathbf{n}=\mathbf{3 4} \\
{\left[\mathbf{N}^{*} \mathbf{m m}^{-2}\right]}\end{array}$ & $\begin{array}{c}\text { Abhebefestig- } \\
\text { keit } \\
\text { "Mitte" } \\
\mathbf{n = 3 1} \\
{\left[\mathbf{N}^{*} \mathbf{m m} \mathbf{m}^{-2}\right]}\end{array}$ & $\begin{array}{c}\text { Abhebefestig- } \\
\text { keit } \\
\text { "Rand" } \\
\mathbf{n}=37 \\
{\left[\mathbf{N}^{*} \mathbf{m m}^{-2}\right]}\end{array}$ \\
\hline Presszeit s & 120 & 120 & 120 & 120 & 120 \\
\hline Median Z & 1,65 & 1,65 & 1,66 & 1,63 & 1,68 \\
\hline $\begin{array}{l}\text { Arithmetisches } \\
\text { Mittel } \boldsymbol{\mu}\end{array}$ & 1,71 & 1,67 & 1,74 & 1,65 & 1,75 \\
\hline $\begin{array}{l}\text { Standardab- } \\
\text { weichung S }\end{array}$ & 0,27 & 0,28 & 0,25 & 0,30 & 0,23 \\
\hline Minimum & 1,05 & 1,05 & 1,35 & 1,05 & 1,38 \\
\hline Maximum & 2,16 & 2,16 & 2,12 & 2,13 & 2,16 \\
\hline
\end{tabular}

Trotz der erheblichen Differenz zwischen den Extremwerten deuten die Darstellungen der Lage- und Streuparameter in Abbildung 4-9 sowie die festgestellten Standardabweichungen darauf hin, dass sich die Fmax zwischen den einzelnen Lagen in den Probeplatten nicht signifikant voneinander unterscheiden.

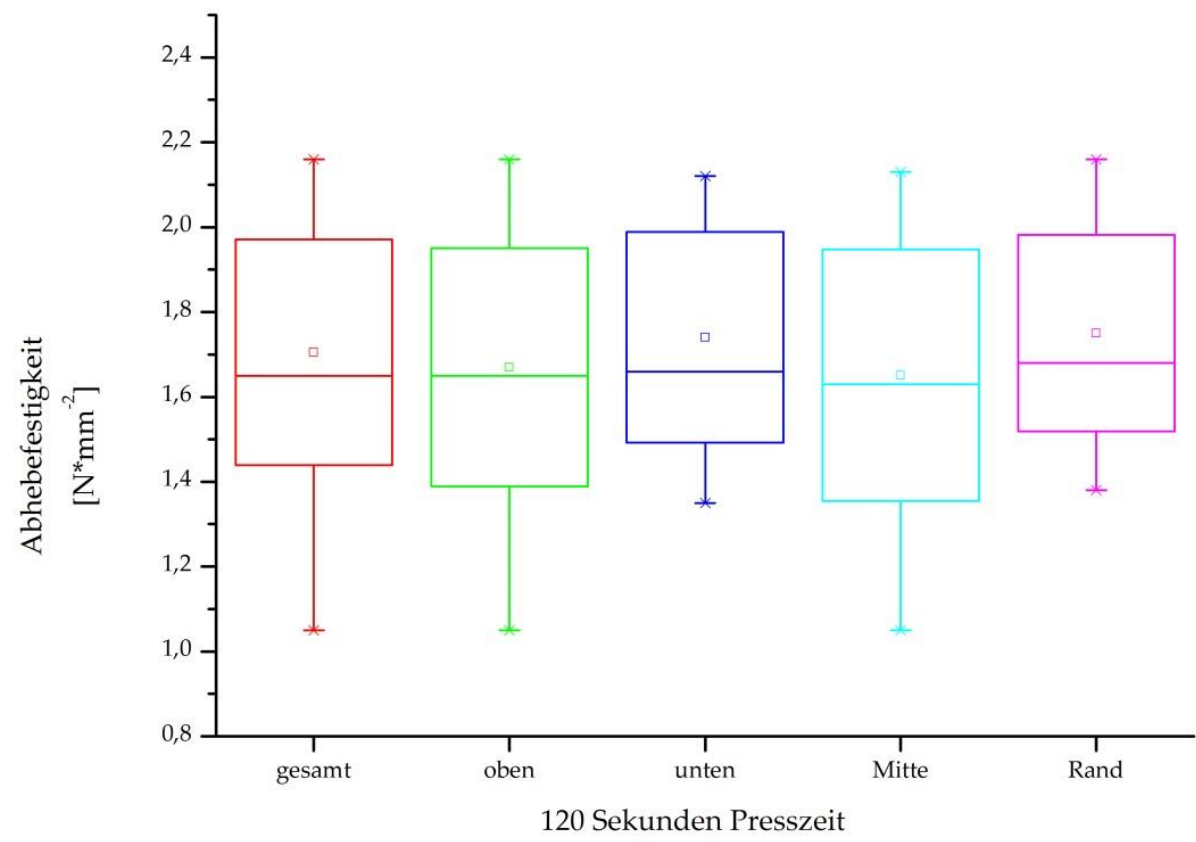

Abbildung 4-9: Boxplots zur Abhebefestigkeit von Erlenfurnier auf PVC bei 120 Sekunden Presszeit, sortiert nach Gesamtergebnis sowie der Probenlage innerhalb der Platten. 
Die statistische Auswertung mittels ANOVA bestätigt diese Annahme. Bei einem Signifikanzniveau von 0,05 unterscheiden sich die Mittelwerte der Grundgesamtheiten nicht signifikant voneinander. Somit muss $\mathrm{H}_{0}$ angenommen und $\mathrm{H}_{1}$ verworfen werden.

\section{Presszeit 180 Sekunden}

Bei einer Presszeit von $180 \mathrm{~s}$ wurde wie aus Tabelle 4-9 ersichtlich, im Mittel eine maximale Bruchkraft (Fmax) von $\bar{x}=1,49 \mathrm{~N}^{*} \mathrm{~mm}^{-2}$ bei einer Standardabweichung von $0,37 \mathrm{~N}^{*} \mathrm{~mm}^{-2}$ gemessen. Wie weiterhin dieser Tabelle zu entnehmen ist, differieren die arithmetischen Mittelwerte der Fmax zwischen den einzelnen Lagen in den Probeplatten um maximal 5,23\% bezogen auf den höchsten Mittelwert von $1,53 \mathrm{~N}^{*} \mathrm{~mm}^{-2}$ im Lagebereich „unten“. Die Extrema liegen mit einer Differenz von $1,59 \mathrm{~N}^{*} \mathrm{~mm}^{-2}$ deutlich auseinander.

Tabelle 4-9: Ergebnisse der Abhebefestigkeitsprüfung von Erlenfurnier auf PVC bei 180 Sekunden Presszeit, differenziert nach dem Gesamtergebnis und den jeweiligen Lagen der Probekörper in den Probeplatten.

\begin{tabular}{|l|c|c|c|c|c|}
\hline Parameter & $\begin{array}{c}\text { Abhebefestig- } \\
\text { keit } \\
\text { "gesamt" } \\
\mathbf{n = 5 0} \\
{\left[\mathbf{N}^{*} \mathbf{m m}^{-2}\right]}\end{array}$ & $\begin{array}{c}\text { Abhebefestig- } \\
\text { keit } \\
\text { "oben" } \\
\mathbf{n}=\mathbf{2 4} \\
{\left[\mathbf{N}^{*} \mathbf{m m}^{-2}\right]}\end{array}$ & $\begin{array}{c}\text { Abhebefestig- } \\
\text { keit } \\
\text { unten" } \\
\mathbf{n = 2 6} \\
{\left[\mathbf{N}^{*} \mathbf{m m}^{-2}\right]}\end{array}$ & $\begin{array}{c}\text { Abhebefestig- } \\
\text { keit } \\
\text { "Mitte" } \\
\mathbf{n = 2 4} \\
{\left[\mathbf{N}^{*} \mathbf{m m}^{-2}\right]}\end{array}$ & $\begin{array}{c}\text { Abhebefestig- } \\
\text { keit } \\
\text { "Rand" } \\
\mathbf{n = 2 6} \\
{\left[\mathbf{N}^{*} \mathbf{m m}^{-2}\right]}\end{array}$ \\
\hline Presszeit s & 180 & 180 & 180 & 180 & 180 \\
\hline Median Z & 1,55 & 1,54 & 1,56 & 1,55 & 1,55 \\
\hline $\begin{array}{l}\text { Arithmetisches } \\
\text { Mittel } \boldsymbol{\mu}\end{array}$ & 1,49 & 1,45 & 1,53 & 1,48 & 1,50 \\
\hline $\begin{array}{l}\text { Standardab- } \\
\text { weichung S }\end{array}$ & 0,37 & 0,31 & 0,41 & 0,32 & 0,41 \\
\hline Minimum & 0,61 & 0,61 & 0,66 & 0,61 & 0,66 \\
\hline Maximum & 2,20 & 1,97 & 2,20 & 1,95 & 2,20 \\
\hline
\end{tabular}

Die beiden aufzustellenden Hypothesen lauten:

$H_{0}$ : Die jeweiligen arithmetischen Mittelwerte der $F_{\max }$, bezogen auf die Lage der Probekörper in der Prüfplatte, unterscheiden sich nicht.

$H_{1}$ : Die jeweiligen arithmetischen Mittelwerte der $F_{\max }$, bezogen auf die Lage der Probekörper in der Prüfplatte, unterscheiden sich signifikant.

Betrachtet man die in Abbildung 4-10 dargestellten Lage- und Streuparameter sowie die festgestellten Standardabweichungen ist trotz der stark differenten Extrema davon auszugehen, dass sich die Fmax zwischen den einzelnen Lagen in den Probeplatten nicht signifikant voneinander unterscheiden. 


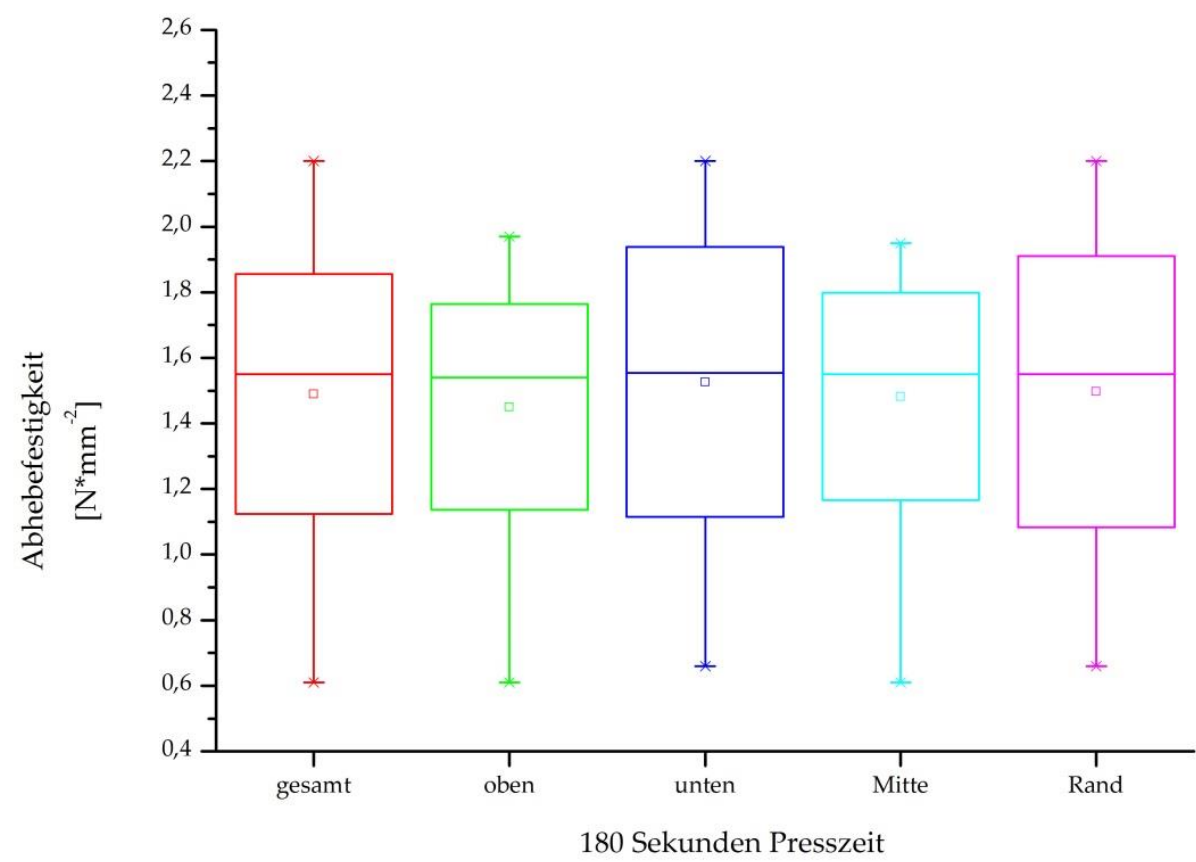

Abbildung 4-10: Boxplots zur Abhebefestigkeit von Erlenfurnier auf PVC bei 180 Sekunden Presszeit, sortiert nach Gesamtergebnis sowie der Probenlage innerhalb der Platten.

Die statistische Auswertung mittels ANOVA bestätigt diese Annahme. Bei einem Signifikanzniveau von 0,05 unterscheiden sich die Mittelwerte der Grundgesamtheiten nicht signifikant voneinander. Somit muss $\mathrm{H}_{0}$ angenommen und $\mathrm{H}_{1}$ verworfen werden.

Presszeit 240 Sekunden

Bei einer Presszeit von $240 \mathrm{~s}$ wurde, wie Tabelle 4-10 zeigt, eine im Mittel maximale Bruchkraft von $\bar{x}=1,74 \mathrm{~N}^{*} \mathrm{~mm}^{-2}$ bei einer Standardabweichung von $0,25 \mathrm{~N}^{*} \mathrm{~mm}^{-2}$ erreicht. Wie weiterhin aus dieser Tabelle ersichtlich, variieren die Werte der mittleren Fmax zwischen den einzelnen Lagen in den Probeplatten um maximal 1,14\%. Die Differenz der Extrema hingegen beträgt $1,06 \mathrm{~N}^{*} \mathrm{~mm}^{-2}$, bei einem Minimum von $1,16 \mathrm{~N}^{*} \mathrm{~mm}^{-2}$ und einem Maximum von 2,22 $\mathrm{N}^{*} \mathrm{~mm}^{-2}$.

Die beiden aufzustellenden Hypothesen lauten:

$H_{0}$ : Die jeweiligen arithmetischen Mittelwerte der $F_{\max }$, bezogen auf die Lage der Probekörper in der Prüfplatte, unterscheiden sich nicht.

$H_{1}$ : Die jeweiligen arithmetischen Mittelwerte der $F_{\max }$, bezogen auf die Lage der Probekörper in der Prüfplatte, unterscheiden sich signifikant. 
Tabelle 4-10: Ergebnisse der Abhebefestigkeitsprüfung von Erlenfurnier auf PVC bei 240 Sekunden Presszeit, differenziert nach dem Gesamtergebnis und den jeweiligen Lagen der Probekörper in den Probeplatten.

\begin{tabular}{|l|c|c|c|c|c|}
\hline Parameter & $\begin{array}{c}\text { Abhebefestig- } \\
\text { keit } \\
\text { "gesamt" } \\
\mathbf{n = 7 3} \\
{\left[\mathbf{N}^{*} \mathbf{m m}^{-2}\right]}\end{array}$ & $\begin{array}{c}\text { Abhebefestig- } \\
\text { keit } \\
\text { "oben" } \\
\mathbf{n = 3 8} \\
{\left[\mathbf{N}^{*} \mathbf{m m}^{-2}\right]}\end{array}$ & $\begin{array}{c}\text { Abhebefestig- } \\
\text { keit } \\
\text { "unten" } \\
\mathbf{n = 3 5} \\
{\left[\mathbf{N}^{*} \mathbf{m m}^{-2}\right]}\end{array}$ & $\begin{array}{c}\text { Abhebefestig- } \\
\text { keit } \\
\text { "Mitte" } \\
\mathbf{n = 3 5} \\
{\left[\mathbf{N}^{*} \mathbf{m m}^{-2}\right]}\end{array}$ & $\begin{array}{c}\text { Abhebefestig- } \\
\text { keit } \\
\text { "Rand" } \\
\mathbf{n = 3 8} \\
{\left[\mathbf{N}^{*} \mathbf{m m}^{-2}\right]}\end{array}$ \\
\hline Presszeit s & 240 & 240 & 240 & 240 & 240 \\
\hline Median Z & 1,82 & 1,74 & 1,82 & 1,84 & 1,71 \\
\hline $\begin{array}{l}\text { Arithmetisches } \\
\text { Mittel } \boldsymbol{\mu}\end{array}$ & 1,74 & 1,74 & 1,74 & 1,75 & 1,73 \\
\hline $\begin{array}{l}\text { Standardab- } \\
\text { weichung S }\end{array}$ & 0,25 & 0,26 & 0,24 & 0,25 & 0,24 \\
\hline Minimum & 1,16 & 1,23 & 1,16 & 1,17 & 1,16 \\
\hline Maximum & 2,22 & 2,22 & 2,12 & 2,22 & 2,12 \\
\hline
\end{tabular}

Aufgrund der minimalen Differenz der Festigkeitswerte, der Betrachtung der Lageund Streuparameter in Abbildung 4-11 sowie der errechneten Standardabweichungen ist davon auszugehen, dass sich die Fmax zwischen den einzelnen Lagen in den Probeplatten nicht signifikant voneinander unterscheiden.

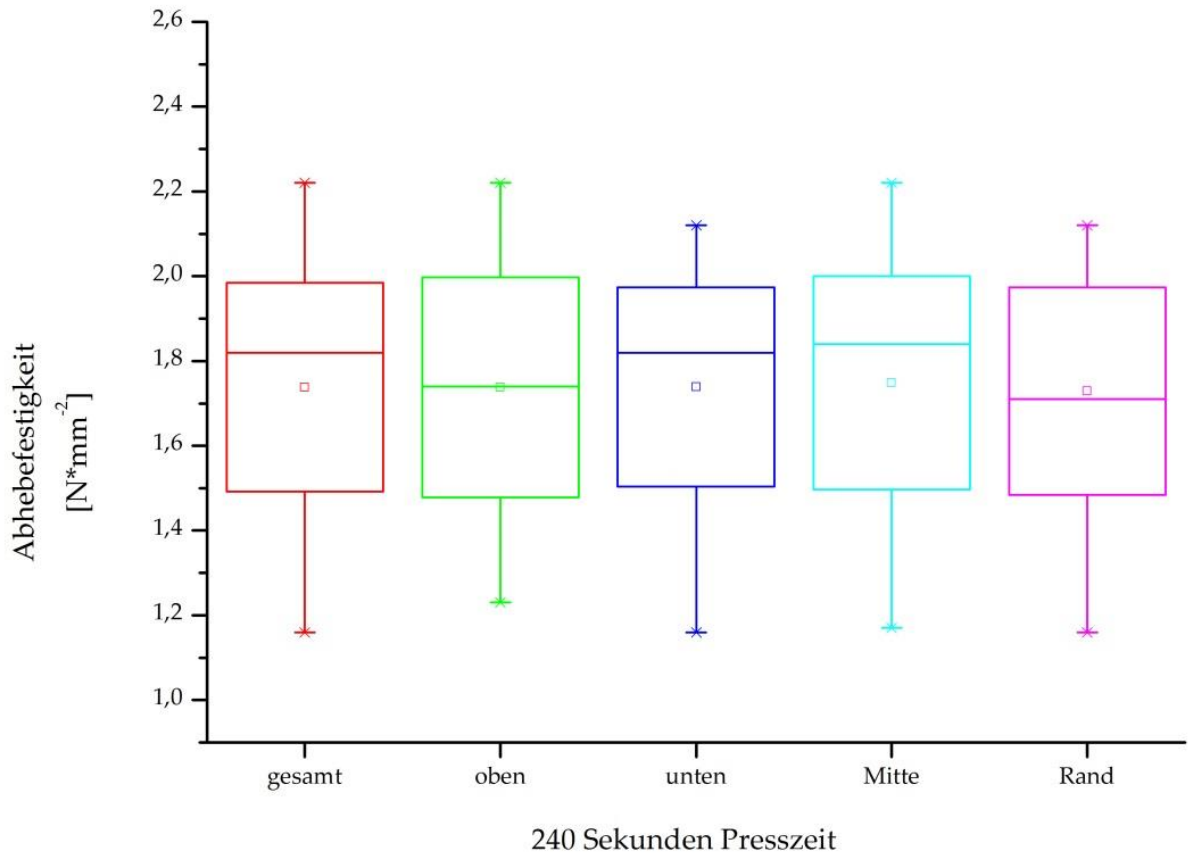

Abbildung 4-11: Boxplots zur Abhebefestigkeit von Erlenfurnier auf PVC bei 240 Sekunden Presszeit, sortiert nach Gesamtergebnis sowie der Probenlage innerhalb der Platten.

Diese Annahme bestätigt auch die statistische Auswertung mittels ANOVA. Bei einem Signifikanzniveau von 0,05 unterscheiden sich die Mittelwerte der Grundgesamtheiten nicht signifikant voneinander. Somit muss $\mathrm{H}_{0}$ angenommen und $\mathrm{H}_{1}$ verworfen werden. 


\section{Vergleich der unterschiedlichen Presszeiten}

Tabelle 4-11 gibt einen Überblick über die Entwicklung der maximalen Abhebefestigkeiten bei den verschiedenen beprobten Presszeiten. Die Bezeichnungen "60s", "90s" usw. beziehen sich auf die jeweilige Presszeit. Die angegeben Werte werden lageunabhängig betrachtet und beziehen sich auf die Gesamtheit der pro Presszeit genommenen Probekörper.

Wie Tabelle 4-11 zu entnehmen ist, entwickeln sich die Werte der Fmax über die Länge der Pressdauer indifferent, was deutlich in Abbildung 4-12 zu erkennen ist.

Während die Fmax bei einer Presszeit von $60 \mathrm{~s}$ mit $\bar{x}=1,25 \mathrm{~N}^{*} \mathrm{~mm}^{-2}$, bei einer errechneten Standardabweichung von $0,31 \mathrm{~N}^{*} \mathrm{~mm}^{-2}$, gemessen wurde, fällt die Fmax bei einer Presszeit von $90 \mathrm{~s}$ auf $\bar{x}=1,17 \mathrm{~N}^{*} \mathrm{~mm}^{-2}\left(\mathrm{~S}=0,28 \mathrm{~N}^{*} \mathrm{~mm}^{-2}\right)$. Die maximale Bruchkraft steigt bei $120 \mathrm{~s}$ Presszeit um $0,54 \mathrm{~N}^{*} \mathrm{~mm}^{-2}$ auf $1,71 \mathrm{~N}^{*} \mathrm{~mm}^{-2}\left(\mathrm{~S}=0,27 \mathrm{~N}^{*} \mathrm{~mm}^{-2}\right)$ an. Bei einer Presszeit von $180 \mathrm{~s}$ fällt die Fmax hingegen wieder um $0,22 \mathrm{~N}^{*} \mathrm{~mm}^{-2}$ auf $1,49 \mathrm{~N}^{*} \mathrm{~mm}^{-2}\left(\mathrm{~S}=0,37 \mathrm{~N}^{*} \mathrm{~mm}^{-2}\right)$ ab, um bei $240 \mathrm{~s}$ wieder um $0,25 \mathrm{~N}^{*} \mathrm{~mm}^{-2}$ auf $1,74 \mathrm{~N}^{*} \mathrm{~mm}^{-2}$ $\left(\mathrm{S}=0,25 \mathrm{~N}^{*} \mathrm{~mm}^{-2}\right)$ anzusteigen.

Tabelle 4-11: Vergleichende Ergebnisse der Abhebefestigkeitsprüfungen von Erlenfurnier auf PVC bei verschiedenen Presszeiten.

\begin{tabular}{|l|c|c|c|c|c|}
\hline Parameter & $\begin{array}{c}\text { Abhebefestig- } \\
\text { keit } \\
\text { "60s" } \\
\mathbf{n = 5 7} \\
{\left[\mathbf{N}^{*} \mathbf{m m}^{-2}\right]}\end{array}$ & $\begin{array}{c}\text { Abhebefestig- } \\
\text { keit } \\
\mathbf{9 0 5 "} \\
\mathbf{n = 6 1} \\
{\left[\mathbf{N}^{*} \mathbf{m m}^{-2}\right]}\end{array}$ & $\begin{array}{c}\text { Abhebefestig- } \\
\text { keit } \\
\mathbf{1 2 0 s "} \\
\mathbf{n = 6 8} \\
{\left[\mathbf{N}^{*} \mathbf{m m}^{-2}\right]}\end{array}$ & $\begin{array}{c}\text { Abhebefestig- } \\
\text { keit } \\
\text { "180s" } \\
\mathbf{n = 5 0} \\
{\left[\mathbf{N}^{*} \mathbf{m m}^{-2}\right]}\end{array}$ & $\begin{array}{c}\text { Abhebefestig- } \\
\text { keit } \\
\text { "240s" } \\
\mathbf{n = 7 3} \\
{\left[\mathbf{N}^{*} \mathbf{m m}^{-2}\right]}\end{array}$ \\
\hline Median Z & 1,24 & 1,18 & 1,65 & 1,55 & 1,82 \\
\hline $\begin{array}{l}\text { Arithmetisches } \\
\text { Mittel } \boldsymbol{\mu}\end{array}$ & 1,25 & 1,17 & 1,71 & 1,49 & 1,74 \\
\hline $\begin{array}{l}\text { Standardab- } \\
\text { weichung S }\end{array}$ & 0,31 & 0,28 & 0,27 & 0,37 & 0,25 \\
\hline Minimum & 0,46 & 0,58 & 1,05 & 0,61 & 1,16 \\
\hline Maximum & 1,84 & 1,82 & 2,16 & 2,20 & 2,22 \\
\hline
\end{tabular}

Die Extremwerte schwanken über alle Versuchsreihen hinweg vom Minimum mit gemessenen $0,46 \mathrm{~N}^{*} \mathrm{~mm}^{-2}$ zum Maximum von gemessenen $2,22 \mathrm{~N}^{*} \mathrm{~mm}^{-2}$. 


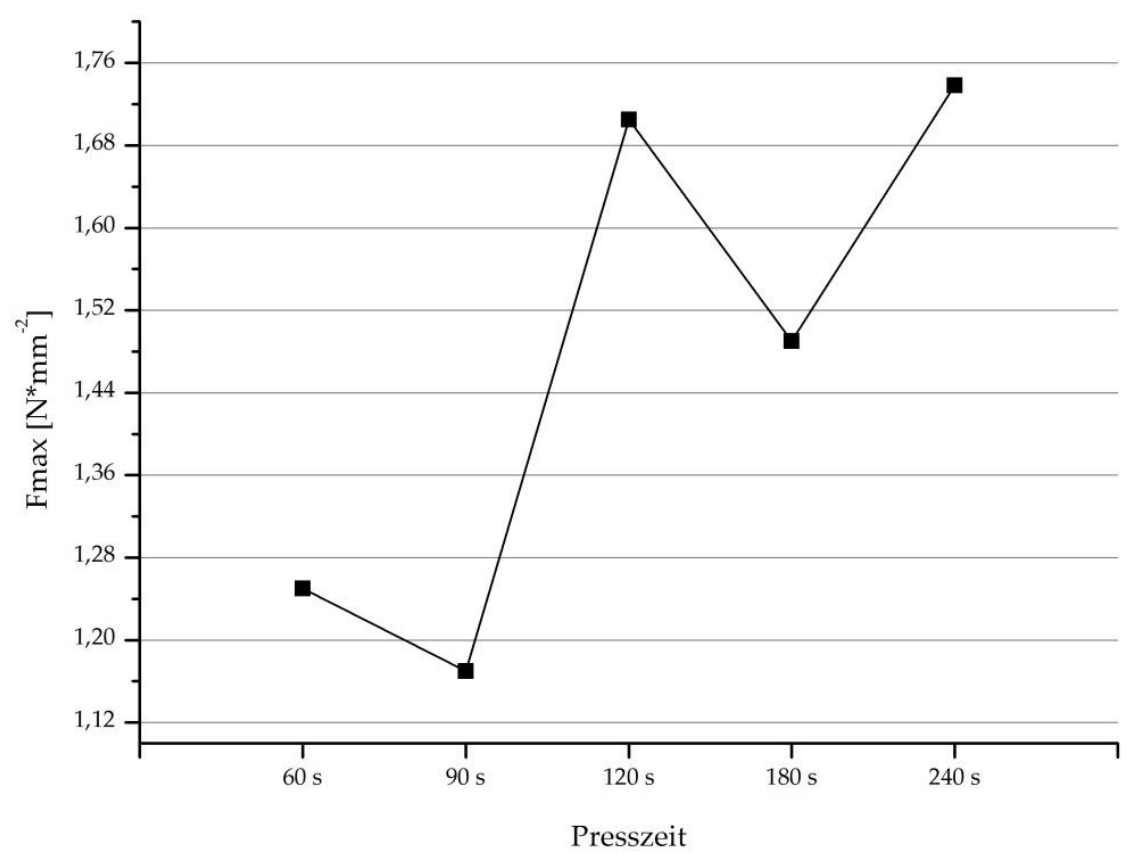

Abbildung 4-12: Entwicklung der Abhebefestigkeit von Erlenfurnier auf PVC in Relation zu den Presszeiten.

Wie aus Abbildung 4-13 ersichtlich, teilen sich die Festigkeitswerte in zwei Gruppen. Eine mit deutlich niedrigeren aber nahe beieinander liegenden Werten sowie eine Gruppe mit höheren Werten und ebenfalls nahe beieinander liegenden Werten.

Es werden wie auch bei den vorherigen Untersuchungen folgende Hypothesen aufgestellt:

$\mathrm{H}_{0}$ : Die jeweiligen arithmetischen Mittelwerte der $\mathrm{F}_{\max }$, bezogen auf Presszeit, unterscheiden sich nicht.

$H_{1}$ : Die jeweiligen arithmetischen Mittelwerte der $F_{\max }$, bezogen auf die Presszeit, unterscheiden sich signifikant. 


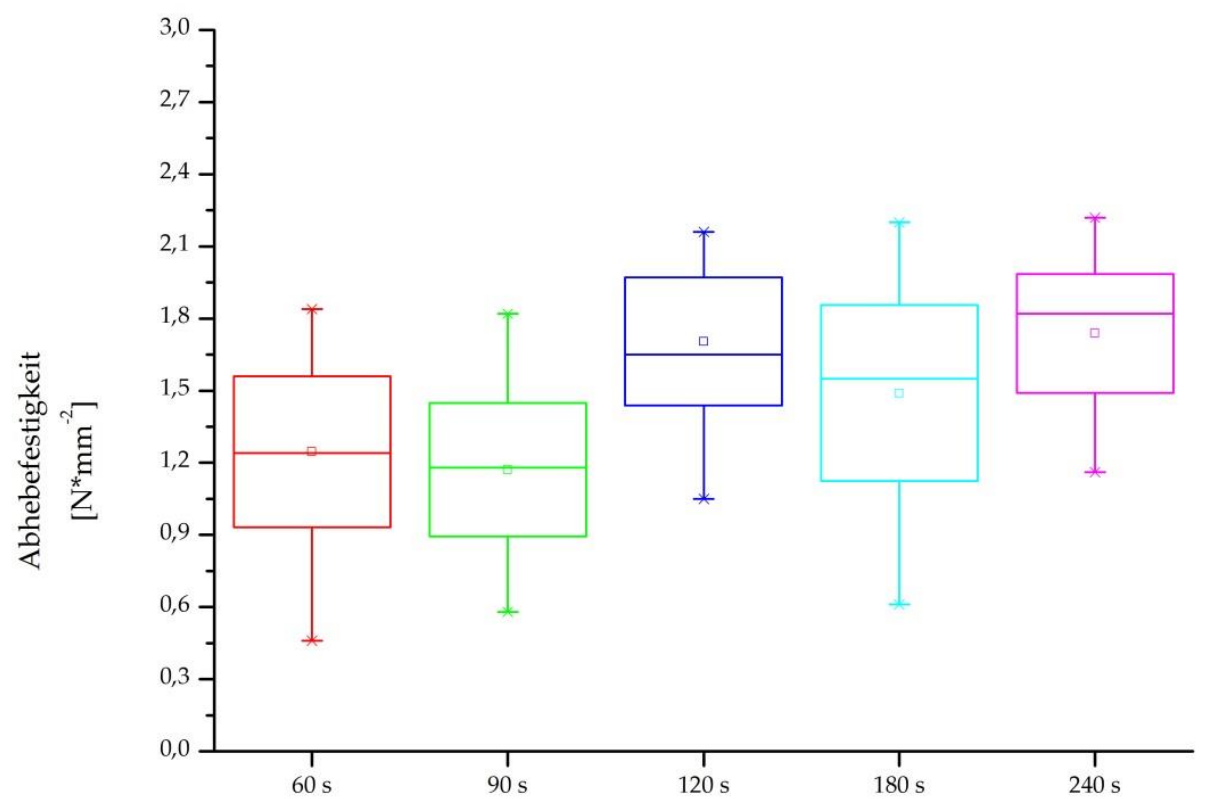

Abbildung 4-13: Vergleichende Boxplots zur Abhebefestigkeit von Erlenfurnier auf PVC, sortiert nach den jeweiligen Presszeiten.

Da es sich bei den Pressezeiten „120 s“ und „240 s" nach Überprüfung um nicht normalverteilte Grundgesamtheiten handelt, wurde ein Kruskal-Wallis-Test zum Vergleich der Mittelwerte durchgeführt.

Das Ergebnis besagt bei einem Signifikanzniveau von 0,05, dass die Stichproben aus verschiedenen Grundgesamtheiten stammen und sich somit mindestens eine Grundgesamtheit signifikant von den Restlichen unterscheidet. Daher muss zunächst $\mathrm{H}_{1}$ grundsätzlich angenommen und $\mathrm{H}_{0}$ verworfen werden.

Da für die vorliegende Arbeit eine möglichst hohe Bruchkraft von Relevanz ist, wurden daher die Werte von "120 s“, "180 s" und "240 s“ noch einmal genauer betrachtet, nachdem zuvor bei einem Signifikanzniveau von 0,05 mittel KruskalWallis-Tests berechnet wurde, dass sich die Mittelwerte von "60 s" und "120 s" signifikant voneinander unterscheiden, wie auch schon deutlich aus Abbildung 4-13 zu interpretieren ist.

Da die Daten von "120 s" und „240 s" aus nicht normalverteilten Grundgesamtheiten stammen, wurde zum Mittelwertvergleich zwischen den drei Datenreihen ein KruskalWallis-Test durchgeführt.

Das Ergebnis der Analyse besagt, wenn auch nach Betrachtung von Abbildung 4-13 kein signifikanter Unterschied zwischen "120 s“ und ,240 s“ zu erwarten war, dass sich die Mittelwerte der drei Messreihen signifikant voneinander unterscheiden. 
Somit muss auch für diese Fälle $\mathrm{H}_{1}$ angenommen und $\mathrm{H}_{0}$ verworfen werden.

\section{Diskussion der Versuchsergebnisse}

Betrachtet man zunächst die Ergebnisse der Abhebefestigkeitsuntersuchungen der einzelnen Versuchsreihen allgemein, so lässt sich feststellen, dass bei sämtlichen Presszeiten die nach DIN EN 312 geforderte minimale Abhebefestigkeit von $0,80 \mathrm{~N}^{*} \mathrm{~mm}^{-2}$ deutlich überschritten und das Mindestziel somit erreicht wurde. In den Versuchsreihen "120 s“ und "240 s“ konnte sogar die von Niemz und Sonderegger (2017) beschriebene maximale Abhebefestigkeit von Spanplatten in Höhe von $1,60 \mathrm{~N}^{*} \mathrm{~mm}^{-2}$ überschritten werden. Wenn auch ein direkter Vergleich mit Spanplatten nicht möglich ist, so kann jedoch bei dem Versuch die Festigkeitswerte des in dieser Arbeit entwickelten Werkstoffes einzuordnen von einer sehr guten Adhäsion zwischen Furnier und PVC-Folie gesprochen werden.

Da Erle als gut zu verleimen gilt Sachsse (1984), wurde schon vor Versuchsbeginn mit zufriedenstellenden Ergebnissen gerechnet. Auch Grosser (2003) beschreibt Leimverbindungen von Erlenholz als besonders fest.

Betrachtet man nun die Untersuchungen innerhalb der einzelnen Versuchsreihen (Presszeiten), wurde bei keiner einzelnen Reihe ein lageabhängiger signifikanter Unterschied zwischen den Mittelwerten festgesellt.

Aufgrund dieser Datenlage lässt sich darlegen, dass die Oberflächenstruktur der PVCFolie, die eine rauere und eine glattere Seite aufweist, keinen Einfluss auf die Abhebefestigkeit des Verbundwerkstoffes hat.

Weiterhin lässt sich konstatieren, dass die Qualität der Klebung über die gesamte Klebefläche von gleichmäßiger Qualität zu sein scheint.

Auffällig ist, dass die Festigkeitswerte mit zunehmender Presszeit nicht linear zu- oder abnehmen, sondern springen. Während zwischen den Mittelwerten der Versuchsreihen "60 s" und "90 s" kein signifikanter Unterschied vorliegt und dem „Phänomen“ somit keine größere Aufmerksamkeit geschenkt werden muss, ist dies im Falle der Schwankungen zwischen den Presszeiten "120 s“, "180 s" und "240 s" different. Die Mittelwerte dieser Reihen unterscheiden sich signifikant. Im Rahmen der vorliegenden Arbeit konnte dieser Umstand nicht abschließend geklärt werden. Auffällig waren im Falle der Reihe „180 s“ die gehäuft ungültigen Prüfergebnisse aufgrund nicht vollständiger Ablösung des Furniers vom Prüfköper und der damit einhergehende geringere Stichprobenumfang. Zudem fällt die hohe Standardabweichung bei dieser Versuchsreihe auf. Eine mögliche Verunreinigung von Furnier oder PVC-Folie war im Anschluss an die Materialprüfungen nicht mehr zu ermitteln, muss aber unter den gegebenen Bedingungen in Betracht gezogen werden. Alle verwendeten Materialien stammten aus der gleichen Charge wie bei den restlichen Versuchsreihen. Ein Fehler in der Klebefolie kann nahezu ausgeschlossen werden, da diese durch eine zusätzliche Folie vor Verunreinigungen geschützt ist. 
Aufgrund der vorliegenden Daten lässt sich schlussendlich folgern, dass mit $120 \mathrm{~s}$ Presszeit der ideale Produktionsparameter gefunden wurde.

Zwar liegen die Fmax bei $240 \mathrm{~s}$ Presszeit $0,03 \mathrm{~N}^{*} \mathrm{~mm}^{-2}$ signifikant höher als im Falle von 120 s, jedoch rechtfertigt dieser minimale Zugewinn an Festigkeit aus ökonomischen Gründen keine Verdoppelung der Presszeit, da einerseits die Presse länger blockiert wird und damit nicht für eine weitere Produktion zur Verfügung steht und andererseits höhere Produktionskosten durch einen gesteigerten Energiebedarf entstehen.

Für sämtliche weiteren Materialuntersuchungen mit Erle wird aufgrund dieser Ergebnisse die Produktion der Probekörper mit einer Presszeit von $120 \mathrm{~s}$, bei einer Presstemperatur von $120^{\circ} \mathrm{C}$ und einem Pressdruck von $13,96 \mathrm{~N}^{*} \mathrm{~mm}^{-2}$ durchgeführt.

\subsubsection{Untersuchung der Abhebefestigkeit von Eichenfurnier auf PVC-Folie}

Wie bereits in Kapitel 4.3.1 beschrieben, wurden ebenfalls Abhebefestigkeitsprüfungen nach DIN EN 311 durchgeführt, um die Verklebungsqualität des Eichenfurniers mit der PVC-Folie in Form der maximalen Bruchkraft zu ermitteln.

Tabelle 4-12 beschreibt die Herstellungsparameter der untersuchten Platten. Diese sind bis auf geringe Unterschiede in der Furnierdicken, von Eiche (im Mittel 0,58 mm) zu Erle (im Mittel 0,54 mm), identisch. Das Plattenformat bleibt einheitlich bei $750 \mathrm{~mm} \times 300 \mathrm{~mm}$. Die PVC-Folie wurde beidseitig mittels "Collano 46.002" / $100 \mathrm{~g}^{*} \mathrm{~mm}^{-2}$ mit jeweils einem Furnierblatt aus Eiche beklebt.

Tabelle 4-12: Herstellungsparameter des untersuchten Materialverbunds aus Eichenfurnier auf PVCFolie.

\begin{tabular}{|l|l|}
\hline Material & Messerfurniere Eiche PVC-Folie \\
\hline Plattenformat & $750 \mathrm{~mm} \times 300 \mathrm{~mm}$ \\
\hline Furnierdicke & $\overline{\mathrm{x}}=0,58 \mathrm{~mm}$ \\
\hline Foliendicke & $\overline{\mathrm{x}}=0,91 \mathrm{~mm}$ \\
\hline Klebstoff MS-Absperrfurnier & Collano $46.002 / 100 \mathrm{~g}^{*} \mathrm{~m}^{-2}$ \\
\hline Presstemperatur & $120^{\circ} \mathrm{C}$ \\
\hline Pressdruck & $13,96 \mathrm{~N}^{*} \mathrm{~cm}^{-2}$ \\
\hline Schließzeit Presse & $\overline{\mathrm{x}}=8,83 \mathrm{~s}$ \\
\hline
\end{tabular}

Auch in den folgenden Versuchsreihen wurden Presszeiten von 60 s, 90 s, 120 s, 180 s und 240 s. untersucht. Diese werden anschließend auf die Lage der Probekörper in den Platten sowie im Gesamtvergleich betrachtet. 
Die im Folgenden verwendeten Namen der Versuchsreihen „oben“, „unten“, „Mitte“ und "Rand" bezeichnen ihre Entnahmestelle aus den Probeplatten also: Oberseite, Unterseite, Mittel- und Randlage. Als „gesamt“ werden sämtliche zu einer Presszeit gehörigen Messwerte, lageunabhängig, tituliert.

Auch bei dieser Versuchsreihe stammen die Differenzen des Umfangs der Grundgesamtheiten aus fehlerhaften Messungen bzw. Probekörpern.

\section{Presszeit 60 Sekunden}

Bei einer Presszeit von $60 \mathrm{~s}$ wurde wie aus Tabelle 4-13 ersichtlich eine im arithmetischen Mittel maximale Bruchkraft von $\bar{x}=0,91 \mathrm{~N}^{*} \mathrm{~mm}^{-2}$ bei einer Standardabweichung von $0,23 \mathrm{~N}^{*} \mathrm{~mm}^{-2}$ erreicht. Die Extrema liegen bei einem Minimum von $0,44 \mathrm{~N}^{*} \mathrm{~mm}^{-2}$ und einem Maximum von $1,55 \mathrm{~N}^{*} \mathrm{~mm}^{-2}$ mit $1,11 \mathrm{~N}^{*} \mathrm{~mm}^{-2}$ deutlich auseinander. Wie weiterhin aus dieser Tabelle ersichtlich, differieren die Werte der Fmax zwischen den einzelnen Lagen in den Probeplatten um maximal 20 \% bezogen auf den höchsten Mittelwert von 1,01 N*mm² im Lagebereich „Rand“.

Tabelle 4-13: Ergebnisse der Abhebefestigkeitsprüfung von Eichenfurnier auf PVC bei 60 Sekunden Presszeit, differenziert nach dem Gesamtergebnis und den jeweiligen Lagen der Probekörper in den Probeplatten.

\begin{tabular}{|l|c|c|c|c|c|}
\hline Parameter & $\begin{array}{c}\text { Abhebefestig- } \\
\text { keit } \\
\text { "gesamt" } \\
\mathbf{n = 8 0} \\
{\left[\mathbf{N}^{*} \mathbf{m m}^{-2}\right]}\end{array}$ & $\begin{array}{c}\text { Abhebefestig- } \\
\text { keit } \\
\text { "oben" } \\
\mathbf{n = 4 1} \\
{\left[\mathbf{N}^{*} \mathbf{m m}^{-2}\right]}\end{array}$ & $\begin{array}{c}\text { Abhebefestig- } \\
\text { keit } \\
\text { unten" } \\
\mathbf{n = 3 9} \\
{\left[\mathbf{N}^{*} \mathbf{m m}^{-2}\right]}\end{array}$ & $\begin{array}{c}\text { Abhebefestig- } \\
\text { keit } \\
\text { "Mitte" } \\
\mathbf{n = 4 1} \\
{\left[\mathbf{N}^{*} \mathbf{m m}^{-2}\right]}\end{array}$ & $\begin{array}{c}\text { Abhebefestig- } \\
\text { keit } \\
\text { "Rand" } \\
\mathbf{n = 3 9} \\
{\left[\mathbf{N}^{*} \mathbf{m m}^{-2}\right]}\end{array}$ \\
\hline Presszeit s & 60 & 60 & 60 & 60 & 60 \\
\hline Median Z & 0,89 & 0,82 & 1,01 & 0,88 & 0,92 \\
\hline $\begin{array}{l}\text { Arithmetisches } \\
\text { Mittel } \boldsymbol{\mu}\end{array}$ & 0,91 & 0,81 & 1,01 & 0,90 & 0,92 \\
\hline $\begin{array}{l}\text { Standardab- } \\
\text { weichung S }\end{array}$ & 0,23 & 0,19 & 0,23 & 0,23 & 0,24 \\
\hline Minimum & 0,44 & 0,44 & 0,46 & 0,44 & 0,45 \\
\hline Maximum & 1,55 & 1,20 & 1,55 & 1,55 & 1,34 \\
\hline
\end{tabular}

Die beiden aufzustellenden Hypothesen lauten:

$\mathrm{H}_{0}$ : Die jeweiligen arithmetischen Mittelwerte der $F_{\max }$, bezogen auf die Lage der Probekörper in der Prüfplatte, unterscheiden sich nicht.

$H_{1}$ : Die jeweiligen arithmetischen Mittelwerte der $F_{\max }$, bezogen auf die Lage der Probekörper in der Prüfplatte, unterscheiden sich signifikant.

Aufgrund der hohen Differenz der Festigkeitswerte, der errechneten Standardabweichungen sowie der Betrachtung der Lage- und Streuparameter in Abbildung 4-14 ist davon auszugehen, dass sich die Fmax zwischen den einzelnen Lagen in den Probeplatten signifikant voneinander unterscheiden. 


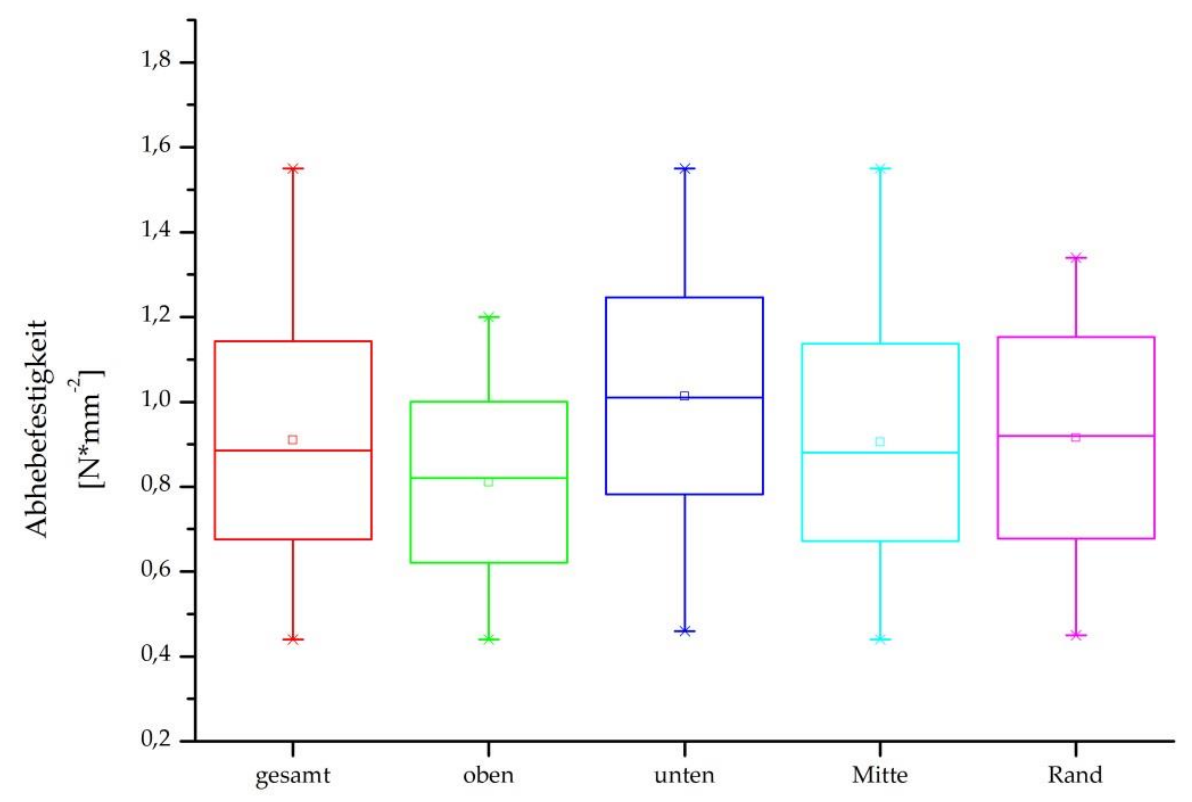

Presszeit 60 Sekunden

Abbildung 4-14: Boxplots zur Abhebefestigkeit von Eichenfurnier auf PVC bei 60 Sekunden Presszeit, sortiert nach Gesamtergebnis sowie der Probenlage innerhalb der Platten.

Die Untersuchung der arithmetischen Mittelwerte mittels ANOVA bestätigt die zuvor getätigte Annahme. Bei einem Signifikanzniveau von 0,05 unterscheidet sich mindestens ein Mittewert signifikant von den Restlichen. Somit muss $\mathrm{H}_{0}$ verworfen und $\mathrm{H}_{1}$ angenommen werden.

Eine weitere durchgeführte ANOVA zwischen den Mittelwerten der einzelnen Lagen zeigt, dass es bei einem Signifikanzniveau von 0,05 die Lagen „oben“ und "unten“ sind, die sich signifikant voneinander unterscheiden, während die Mittelwerte zwischen „Mitte“ und „Rand“ keine signifikanten Unterschiede aufweisen.

\section{Presszeit 90 Sekunden}

Wie Tabelle 4-14 zeigt liegt das arithmetische Mittel der maximalen Bruchkraft bei $\bar{x}=1,22 \mathrm{~N}^{*} \mathrm{~mm}^{-2}$ und einer Standardabweichung von $0,24 \mathrm{~N}^{*} \mathrm{~mm}^{-2}$. Die maximale Differenz der Mittelwerte zwischen den einzelnen Lagen der Probekörper in der Platten liegt bei lediglich $0,03 \mathrm{~N}^{*} \mathrm{~mm}^{-2}$ und somit bei 2,4 \% bezogen auf den höchsten Mittelwert von 1,25 $\mathrm{N}^{*} \mathrm{~mm}^{-2}$. Zwischen den Extremwerten kann ein Unterschied von $1,13 \mathrm{~N}^{*} \mathrm{~mm}^{-2}$ festgestellt werden 
Tabelle 4-14: Ergebnisse der Abhebefestigkeitsprüfung von Eichenfurnier auf PVC bei 90 Sekunden Presszeit, differenziert nach dem Gesamtergebnis und den jeweiligen Lagen der Probekörper in den Probeplatten.

\begin{tabular}{|l|c|c|c|c|c|}
\hline Parameter & $\begin{array}{c}\text { Abhebefestig- } \\
\text { keit } \\
\text { "gesamt" } \\
\mathbf{n}=\mathbf{7 6} \\
{\left[\mathbf{N}^{*} \mathbf{m m}^{-2}\right]}\end{array}$ & $\begin{array}{c}\text { Abhebefestig- } \\
\text { keit } \\
\text { "oben" } \\
\mathbf{n}=\mathbf{3 9} \\
{\left[\mathbf{N}^{*} \mathbf{m m}^{-2}\right]}\end{array}$ & $\begin{array}{c}\text { Abhebefestig- } \\
\text { keit } \\
\text { "unten" } \\
\mathbf{n}=\mathbf{3 7} \\
{\left[\mathbf{N}^{*} \mathbf{m m}^{-2}\right]}\end{array}$ & $\begin{array}{c}\text { Abhebefestig- } \\
\text { keit } \\
\text { "Mitte" } \\
\mathbf{n}=\mathbf{3 8} \\
{\left[\mathbf{N}^{*} \mathbf{m m}^{-2}\right]}\end{array}$ & $\begin{array}{c}\text { Abhebefestig- } \\
\text { keit } \\
\text { "Rand" } \\
\mathbf{n = 3 8} \\
{\left[\mathbf{N}^{*} \mathbf{m m}^{-2}\right]}\end{array}$ \\
\hline Presszeit s & 90 & 90 & 90 & 90 & 90 \\
\hline Median Z & 1,27 & 1,26 & 1,27 & 1,27 & 1,25 \\
\hline $\begin{array}{l}\text { Arithmetisches } \\
\text { Mittel } \boldsymbol{\mu}\end{array}$ & 1,22 & 1,25 & 1,22 & 1,24 & 1,23 \\
\hline $\begin{array}{l}\text { Standardab- } \\
\text { weichung S }\end{array}$ & 0,24 & 0,22 & 0,26 & 0,23 & 0,25 \\
\hline Minimum & 0,54 & 0,74 & 0,54 & 0,74 & 0,54 \\
\hline Maximum & 1,67 & 1,67 & 1,63 & 1,67 & 1,63 \\
\hline
\end{tabular}

Die beiden aufzustellenden Hypothesen lauten:

$H_{0}$ : Die jeweiligen arithmetischen Mittelwerte der $F_{\max }$, bezogen auf die Lage der Probekörper in der Prüfplatte, unterscheiden sich nicht.

$H_{1}$ : Die jeweiligen arithmetischen Mittelwerte der $F_{\max }$, bezogen auf die Lage der Probekörper in der Prüfplatte, unterscheiden sich signifikant.

Aufgrund der sehr geringen Differenz der Mittelwerte und bei Betrachtung der Lage und Streuparameter in Abbildung 4-15 ist davon auszugehen, dass sich die Grundgesamtheiten bezogen auf ihre Mittelwerte nicht signifikant voneinander unterscheiden. 


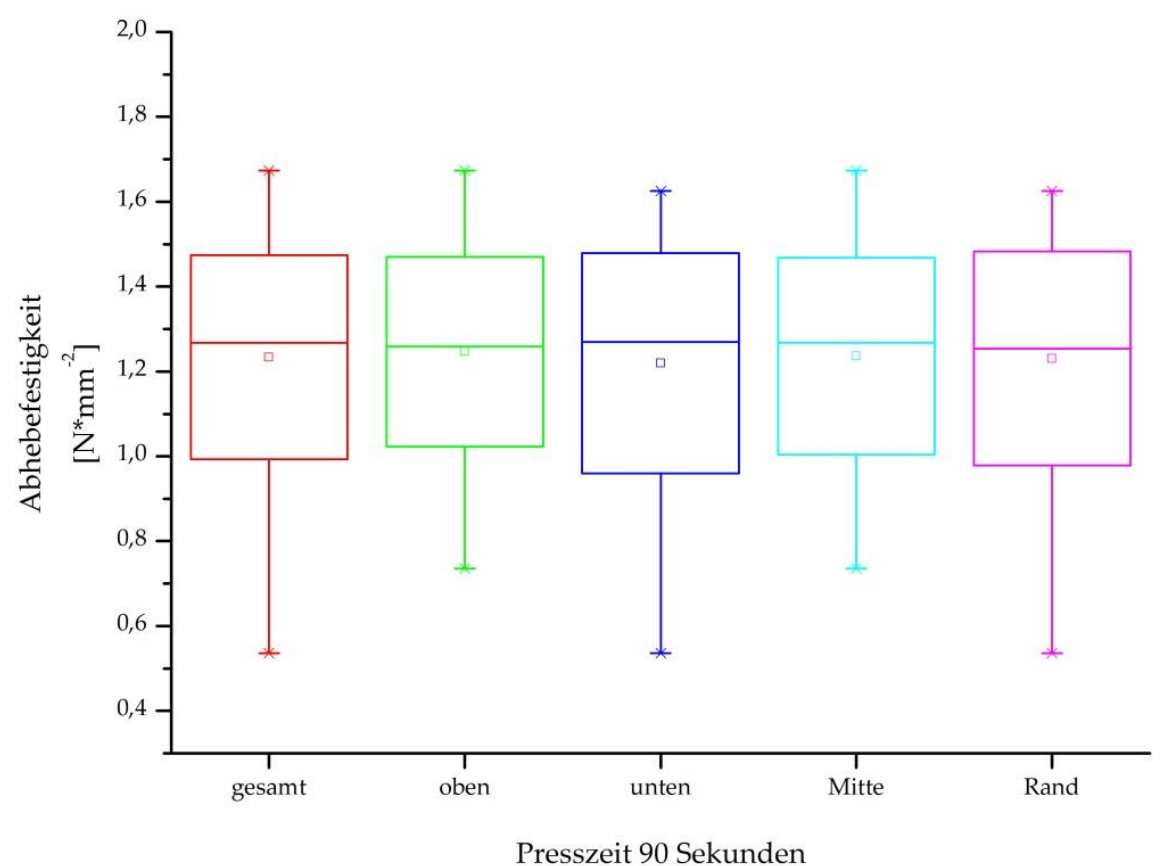

Abbildung 4-15: Boxplots zur Abhebefestigkeit von Eichenfurnier auf PVC bei 90 Sekunden Presszeit, sortiert nach Gesamtergebnis sowie der Probenlage innerhalb der Platten.

Die Untersuchung der Daten mittels ANOVA bestätigt bei einem Signifikanzniveau von 0,05 diese Annahme. Die Mittelwerte der Grundgesamtheiten unterscheiden sich nicht signifikant. Somit muss $\mathrm{H}_{0}$ angenommen und $\mathrm{H}_{1}$ verworfen werden.

\section{Presszeit 120 Sekunden}

Die Lage- und Streuparameter der Abhebefestigkeitsprüfung von Eichenfurnier auf PVC-Folie bei einer Presszeit von 120 s stellen sich wie aus Tabelle 4-15 ersichtlich dar.

Tabelle 4-15: Ergebnisse der Abhebefestigkeitsprüfung von Eichenfurnier auf PVC bei 120 Sekunden Presszeit, differenziert nach dem Gesamtergebnis und den jeweiligen Lagen der Probekörper in den Probeplatten.

\begin{tabular}{|l|c|c|c|c|c|}
\hline Parameter & $\begin{array}{c}\text { Abhebefestig- } \\
\text { keit } \\
\text { "gesamt" } \\
\mathbf{n = 7 4} \\
{\left[\mathbf{N}^{*} \mathbf{m m}-\mathbf{2}\right]}\end{array}$ & $\begin{array}{c}\text { Abhebefestig- } \\
\text { keit } \\
\text { oben" } \\
\mathbf{n = 3 6} \\
{\left[\mathbf{N}^{*} \mathbf{m m}^{-2}\right]}\end{array}$ & $\begin{array}{c}\text { Abhebefestig- } \\
\text { keit } \\
\text { unten" } \\
\mathbf{n}=\mathbf{3 8} \\
{\left[\mathbf{N}^{*} \mathbf{m m}^{-2}\right]}\end{array}$ & $\begin{array}{c}\text { Abhebefestig- } \\
\text { keit } \\
\text { "Mitte" } \\
\mathbf{n = 3 7} \\
{\left[\mathbf{N}^{*} \mathbf{m m}^{-2}\right]}\end{array}$ & $\begin{array}{c}\text { Abhebefestig- } \\
\text { keit } \\
\text { "Rand" } \\
\mathbf{n = 3 7} \\
{\left[\mathbf{N}^{*} \mathbf{m m}^{-2}\right]}\end{array}$ \\
\hline Presszeit s & 120 & 120 & 120 & 120 & 120 \\
\hline Median Z & 1,53 & 1,52 & 1,54 & 1,52 & 1,57 \\
\hline $\begin{array}{l}\text { Arithmetisches } \\
\text { Mittel } \boldsymbol{\mu}\end{array}$ & 1,48 & 1,46 & 1,50 & 1,45 & 1,51 \\
\hline $\begin{array}{l}\text { Standardab- } \\
\text { weichung S }\end{array}$ & 0,26 & 0,26 & 0,25 & 0,28 & 0,23 \\
\hline Minimum & 0,87 & 0,89 & 0,87 & 0,87 & 0,93 \\
\hline Maximum & 1,88 & 1,83 & 1,88 & 1,88 & 1,83 \\
\hline
\end{tabular}


Der Mittelwert der maximalen Bruchkraft über alle Lagen hinweg beträgt $\overline{\mathrm{x}}=1,48 \mathrm{~N}^{*} \mathrm{~mm}^{-2}$ bei einer Standardabweichung von $0,26 \mathrm{~N}^{*} \mathrm{~mm}^{-2}$. Zwischen den einzelnen Lagen differiert das arithmetische Mittel um maximal 0,06 $\mathrm{N}^{*} \mathrm{~mm}^{-2}$. Die Extremwerte differieren bei einem Minimum von $0,87 \mathrm{~N}^{*} \mathrm{~mm}^{-2}$ und einem Maximum von $1,88 \mathrm{~N}^{*} \mathrm{~mm}^{-2}$ um $1,01 \mathrm{~N}^{*} \mathrm{~mm}^{-2}$.

Die beiden aufzustellenden Hypothesen lauten somit:

$H_{0}$ : Die jeweiligen arithmetischen Mittelwerte der $F_{\max }$, bezogen auf die Lage der Probekörper in der Prüfplatte, unterscheiden sich nicht.

$H_{1}$ : Die jeweiligen arithmetischen Mittelwerte der $F_{\max }$, bezogen auf die Lage der Probekörper in der Prüfplatte, unterscheiden sich signifikant.

Aufgrund der Differenz zwischen den Mittelwerten, der errechnete Standardabweichungen und bei Betrachtung der Lage und Streuparameter in Abbildung 4-16 ist davon auszugehen, dass sich die Grundgesamtheiten bezogen auf ihre Mittelwerte nicht signifikant voneinander unterscheiden.

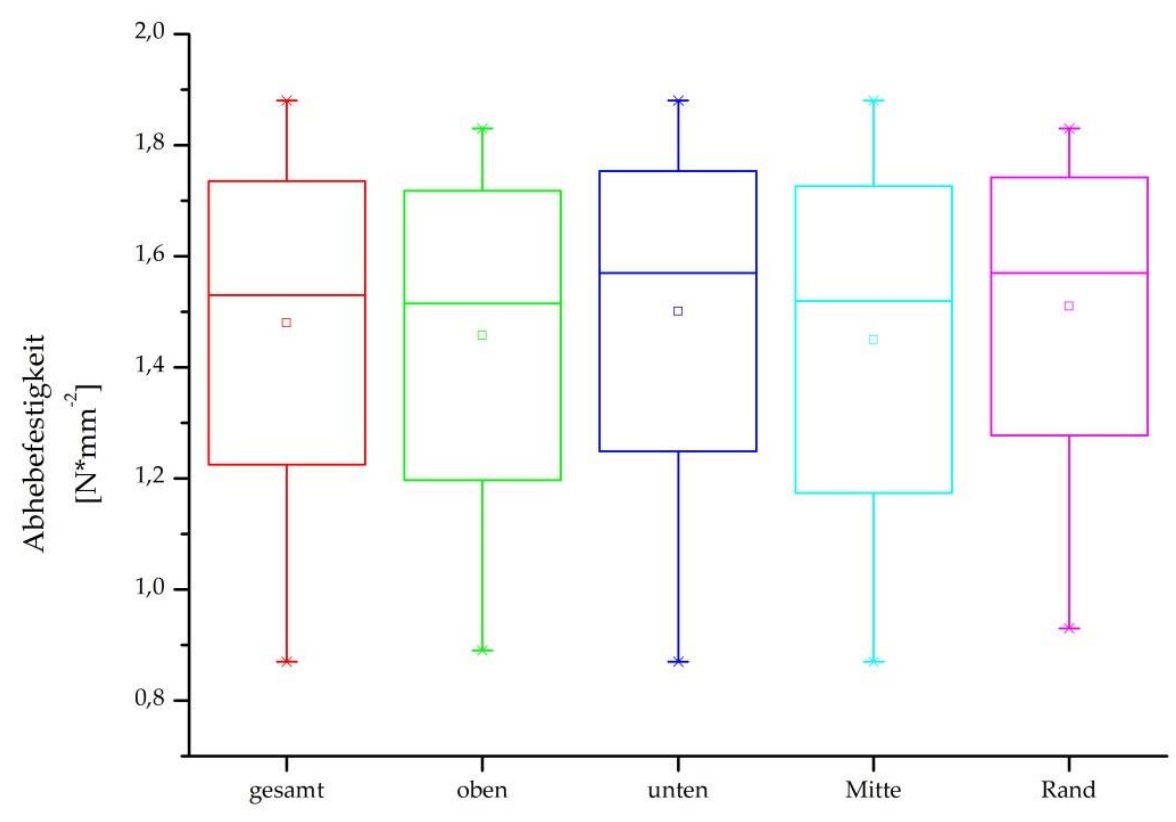

Presszeit 120 Sekunden

\begin{abstract}
Abbildung 4-16: Boxplots zur Abhebefestigkeit von Eichenfurnier auf PVC bei 120 Sekunden Presszeit, sortiert nach Gesamtergebnis sowie der Probenlage innerhalb der Platten.

Die statistische Auswertung mittels ANOVA bestätigt die o.g. Annahme. Die Mittelwerte der Grundgesamtheiten unterscheiden sich bei einem Signifikanzniveau von 0,05 nicht signifikant. Somit muss $\mathrm{H}_{0}$ angenommen und $\mathrm{H}_{1}$ verworfen werden.
\end{abstract}




\section{Presszeit 180 Sekunden}

Wie die Darstellung der Lage- und Streuparameter in Tabelle 4-16 zeigt, liegt der Mittelwert der maximalen Bruchkraft über alle Lagen bei $\bar{x}=1,64 \mathrm{~N}^{*} \mathrm{~mm}^{-2}$ und einer Standardabweichung von $0,18 \mathrm{~N}^{*} \mathrm{~mm}^{-2}$. Die höchste Differenz zwischen den arithmetischen Mittelwerten der einzelnen Lagen beträgt $0,14 \mathrm{~N}^{*} \mathrm{~mm}^{-2}$ und stellt somit eine Abweichung von 8,2 \% bezogen auf den höchsten Mittelwert von 1,71 $\mathrm{N}^{*} \mathrm{~mm}^{-2}$ in der Lage „Rand“ dar. Die Extremwerte differieren um $0,84 \mathrm{~N}^{*} \mathrm{~mm}^{-2}$ bei einem gemessenen Minimum von 1,20 $\mathrm{N}^{*} \mathrm{~mm}^{-2}$ und einem Maximum von 2,04 $\mathrm{N}^{*} \mathrm{~mm}^{-2}$.

Tabelle 4-16: Ergebnisse der Abhebefestigkeitsprüfung von Eichenfurnier auf PVC bei 180 Sekunden Presszeit, differenziert nach dem Gesamtergebnis und den jeweiligen Lagen der Probekörper in den Probeplatten.

\begin{tabular}{|l|c|c|c|c|c|}
\hline Parameter & $\begin{array}{c}\text { Abhebefestig- } \\
\text { keit } \\
\text { "gesamt" } \\
\mathbf{n = 8 0} \\
{\left[\mathbf{N}^{*} \mathbf{m m}^{-2}\right]}\end{array}$ & $\begin{array}{c}\text { Abhebefestig- } \\
\text { keit } \\
\text { "oben" } \\
\mathbf{n = 4 0} \\
{\left[\mathbf{N}^{*} \mathbf{m m}^{-2}\right]}\end{array}$ & $\begin{array}{c}\text { Abhebefestig- } \\
\text { keit } \\
\text { "unten" } \\
\mathbf{n}=\mathbf{4 0} \\
{\left[\mathbf{N}^{*} \mathbf{m m}^{-2}\right]}\end{array}$ & $\begin{array}{c}\text { Abhebefestig- } \\
\text { keit } \\
\text { "Mitte" } \\
\mathbf{n = 4 0} \\
{\left[\mathbf{N}^{*} \mathbf{m m} \mathbf{m}^{-2}\right]}\end{array}$ & $\begin{array}{c}\text { Abhebefestig- } \\
\text { keit } \\
\text { "Rand" } \\
\mathbf{n}=\mathbf{4 0} \\
{\left[\mathbf{N}^{*} \mathbf{m m}^{-2}\right]}\end{array}$ \\
\hline Presszeit s & 180 & 180 & 180 & 180 & 180 \\
\hline Median Z & 1,64 & 1,64 & 1,64 & 1,57 & 1,72 \\
\hline $\begin{array}{l}\text { Arithmetisches } \\
\text { Mittel } \boldsymbol{\mu}\end{array}$ & 1,64 & 1,61 & 1,67 & 1,57 & 1,71 \\
\hline $\begin{array}{l}\text { Standardab- } \\
\text { weichung S }\end{array}$ & 0,18 & 0,16 & 0,20 & 0,17 & 0,17 \\
\hline Minimum & 1,20 & 1,31 & 1,20 & 1,20 & 1,38 \\
\hline Maximum & 2,04 & 1,86 & 2,04 & 2,01 & 2,04 \\
\hline
\end{tabular}

Die beiden aufzustellenden Hypothesen lauten somit:

$H_{0}$ : Die jeweiligen arithmetischen Mittelwerte der $F_{\max }$, bezogen auf die Lage der Probekörper in der Prüfplatte, unterscheiden sich nicht.

$H_{1}$ : Die jeweiligen arithmetischen Mittelwerte der $F_{\max }$, bezogen auf die Lage der Probekörper in der Prüfplatte, unterscheiden sich signifikant.

Aufgrund der Differenz zwischen den gemessenen Mittelwerten, der aufgezeigten Standardabweichungen und bei Betrachtung der Lage- und Streuparameter in Abbildung 4-17 ist davon auszugehen, dass sich die Grundgesamtheiten bezogen auf ihre Mittelwerte signifikant voneinander unterscheiden. 


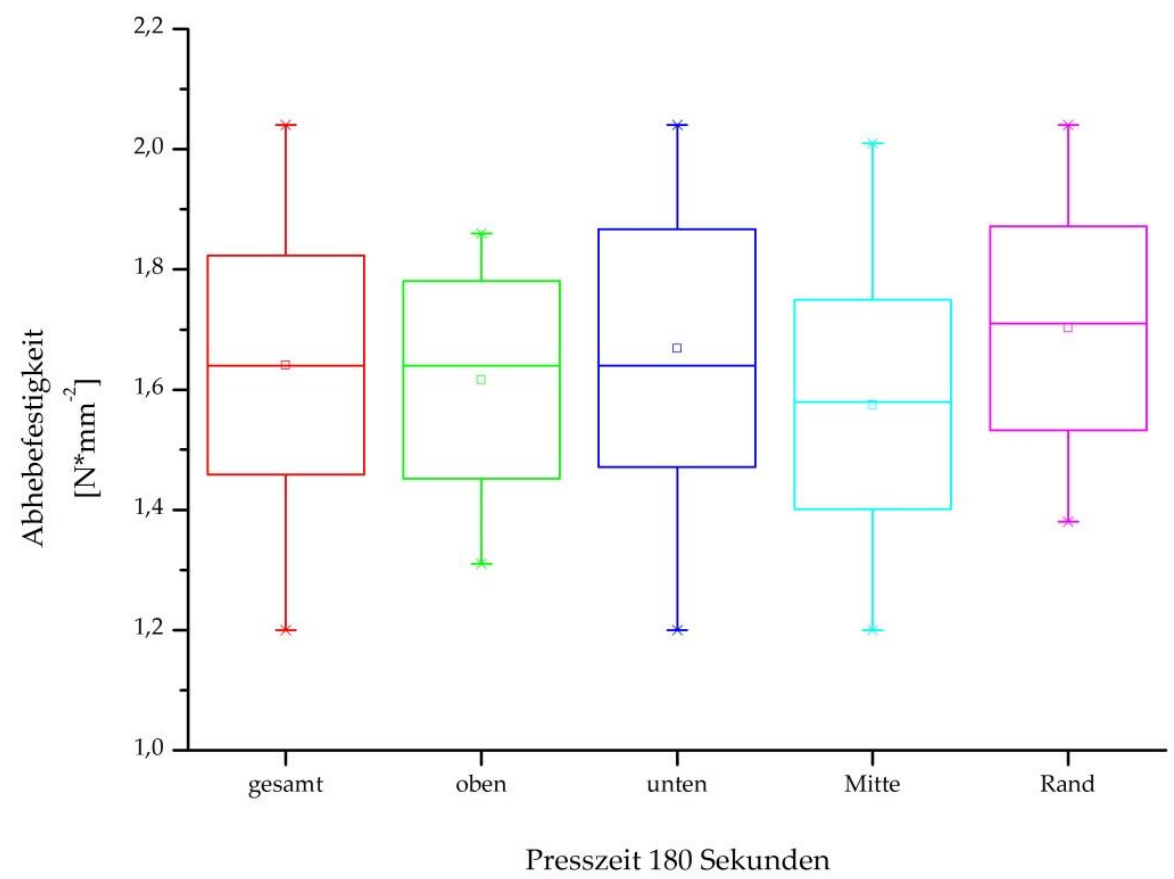

Abbildung 4-17: Boxplots zur Abhebefestigkeit von Eichefurnier auf PVC bei 180 Sekunden Presszeit, sortiert nach Gesamtergebnis sowie der Probenlage innerhalb der Platten.

Die Annahme eines signifikanten Unterschiedes zwischen den Mittelwerten der einzelnen Grundgesamtheiten wird bei einem Signifikanzniveau von 0,05 mittels ANOVA bestätigt. Mindestens ein Mittelwert unterscheidet sich signifikant von den Restlichen. Somit muss $\mathrm{H}_{0}$ verworfen und $\mathrm{H}_{1}$ angenommen werden.

Beim einzelnen Vergleich der Mittelwerte gegeneinander mittels ANOVA ließ sich feststellen, dass sich bei einem Signifikanzniveau von 0,05 die Mittelwerte von "oben“ und „unten“ nicht, diejenigen von „Mitte“ und „Rand“ jedoch signifikant voneinander unterscheiden, worauf auch schon Abbildung 4-17 schließen lässt.

\section{Presszeit 240 Sekunden}

Wie aus Tabelle 4-17 ersichtlich, wurde ein arithmetischer Mittelwert für die maximale Bruchkraft über alle Lagen von $\bar{x}=1,45 \mathrm{~N}^{*} \mathrm{~mm}^{-2}$ bei einer Standardabweichung von $0,28 \mathrm{~N}^{*} \mathrm{~mm}^{-2}$ gemessen. Die höchste Differenz zwischen den lageabhängigen Mittelwerten liegt bei $0,17 \mathrm{~N}^{*} \mathrm{~mm}^{-2}$, was eine Abweichung von 11,04\% bedeutet. Zwischen dem Minimalwert von 0,81 $\mathrm{N}^{*} \mathrm{~mm}^{-2}$ und dem Maximalwert von 2,07 $\mathrm{N}^{*} \mathrm{~mm}^{-2}$ ist eine hohe Abweichung von 1,26 $\mathrm{N}^{*} \mathrm{~mm}^{-2}$ festzustellen. 
Tabelle 4-17: Ergebnisse der Abhebefestigkeitsprüfung von Eichenfurnier auf PVC bei 240 Sekunden Presszeit, differenziert nach dem Gesamtergebnis und den jeweiligen Lagen der Probekörper in den Probeplatten.

\begin{tabular}{|l|c|c|c|c|c|}
\hline Parameter & $\begin{array}{c}\text { Abhebefestig- } \\
\text { keit } \\
\text { "gesamt" } \\
\mathbf{n = 8 0} \\
{\left[\mathbf{N}^{*} \mathbf{m m}^{-2}\right]}\end{array}$ & $\begin{array}{c}\text { Abhebefestig- } \\
\text { keit } \\
\text { "oben" } \\
\mathbf{n = 4 0} \\
{\left[\mathbf{N}^{*} \mathbf{m m}^{-2}\right]}\end{array}$ & $\begin{array}{c}\text { Abhebefestig- } \\
\text { keit } \\
\text { unten" } \\
\mathbf{n = 4 0} \\
{\left[\mathbf{N}^{*} \mathbf{m m}^{-2}\right]}\end{array}$ & $\begin{array}{c}\text { Abhebefestig- } \\
\text { keit } \\
\text { "Mitte" } \\
\mathbf{n = 4 0} \\
{\left[\mathbf{N}^{*} \mathbf{m m}^{-2}\right]}\end{array}$ & $\begin{array}{c}\text { Abhebefestig- } \\
\text { keit } \\
\text { "Rand" } \\
\mathbf{n = 4 0} \\
{\left[\mathbf{N}^{*} \mathbf{m m}^{-2}\right]}\end{array}$ \\
\hline Presszeit $\mathbf{~}$ & 240 & 240 & 240 & 240 & 240 \\
\hline Median Z & 1,49 & 1,36 & 1,56 & 1,42 & 1,52 \\
\hline $\begin{array}{l}\text { Arithmetisches } \\
\text { Mittel } \boldsymbol{\mu}\end{array}$ & 1,45 & 1,37 & 1,54 & 1,40 & 1,51 \\
\hline $\begin{array}{l}\text { Standardab- } \\
\text { weichung S }\end{array}$ & 0,28 & 0,31 & 0,22 & 0,32 & 0,22 \\
\hline Minimum & 0,81 & 0,81 & 0,96 & 0,81 & 1,03 \\
\hline Maximum & 2,07 & 2,07 & 1,99 & 2,07 & 1,88 \\
\hline
\end{tabular}

Die beiden aufzustellenden Hypothesen lauten somit:

$H_{0}$ : Die jeweiligen arithmetischen Mittelwerte der $F_{\max }$, bezogen auf die Lage der Probekörper in der Prüfplatte, unterscheiden sich nicht.

$H_{1}$ : Die jeweiligen arithmetischen Mittelwerte der $F_{\max }$, bezogen auf die Lage der Probekörper in der Prüfplatte, unterscheiden sich signifikant.

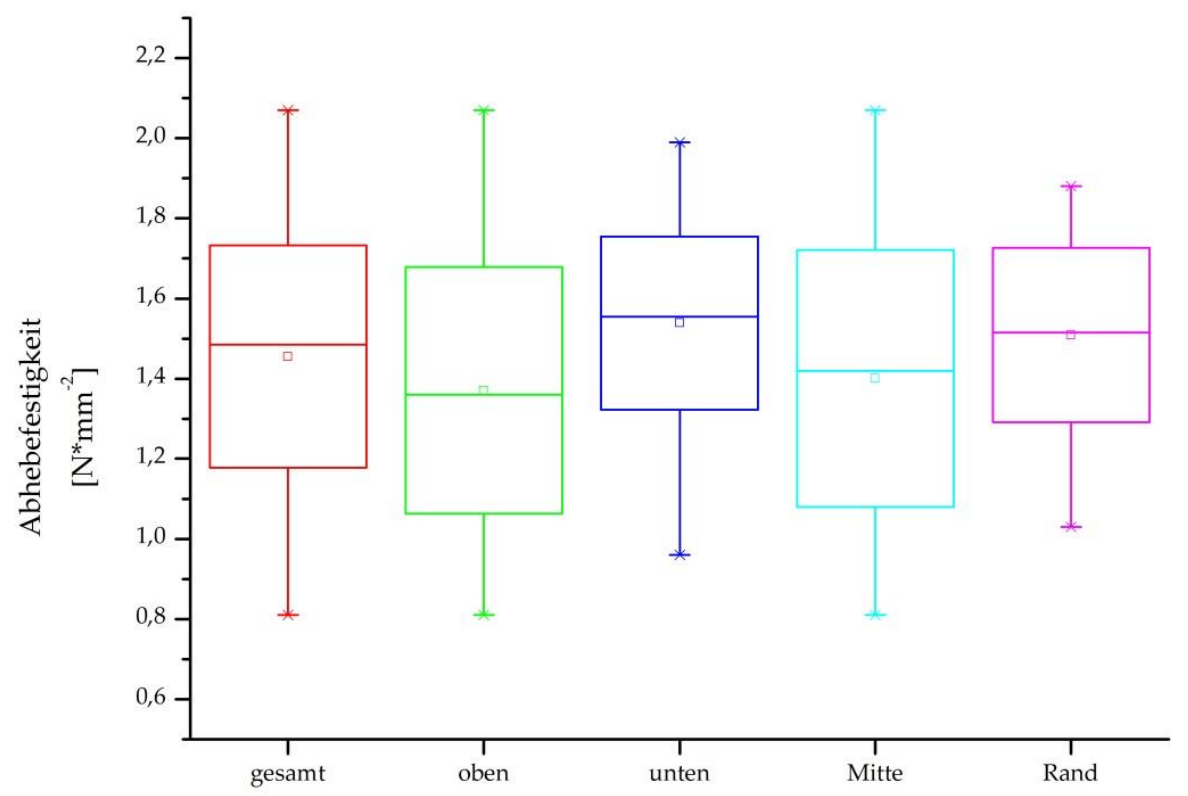

Presszeit 240 Sekunden

Abbildung 4-18: Boxplots zur Abhebefestigkeit von Eichenfurnier auf PVC bei 240 Sekunden Presszeit, sortiert nach Gesamtergebnis sowie der Probenlage innerhalb der Platten. 
Aufgrund der Differenzen zwischen den Mittelwerten, der dazu errechneten Standardabweichungen und bei Betrachtung der Lage und Streuparameter in Abbildung 4-18 ist davon auszugehen, dass sich die Grundgesamtheiten bezogen auf ihre Mittelwerte signifikant voneinander unterscheiden.

Die statistische Auswertung mittels Kruskal-Wallis-Tests bestätigt bei einem Signifikanzniveau von 0,05 die oben getätigte Annahme. Somit muss $\mathrm{H}_{0}$ verworfen und $\mathrm{H}_{1}$ angenommen werden. Mindestens ein Mittelwert unterscheidet sich signifikant von den Restlichen.

Im hier vorliegenden Fall musste ein nicht-parametrischer Test (Kruskal-Wallis-Test) durchgeführt werden, da der Levene-Test auf Varianzhomogenität bei einem Signifikanzniveau von 0,05 negativ ausfiel, die Varianzen der Grundgesamtheit somit heterogen verteilt waren.

Der intensivere Vergleich zwischen den einzelnen Lagen zeigt bei einem Signifikanzniveau von 0,05, dass sich sowohl die Versuchsreihen „oben“ und „unten“ sowie "Mitte“ und „Rand" signifikant voneinander unterscheiden.

\section{Vergleich der unterschiedlichen Presszeiten}

Tabelle 4-18 gibt einen zusammenfassenden Überblick zu den Versuchsergebnissen der Abhebefestigkeitsprüfungen von Eichenfurnier auf PVC bei verschiedenen Presszeiten. Die im Folgenden verwendeten Bezeichnungen "60 s", "90 s" usw. beziehen sich direkt auf eben jene Presszeiten.

Tabelle 4-18: Vergleichende Ergebnisse der Abhebefestigkeitsprüfungen von Eichenfurnier auf PVC bei verschiedenen Presszeiten.

\begin{tabular}{|c|c|c|c|c|c|}
\hline Parameter & $\begin{array}{c}\text { Abhebefestig- } \\
\text { keit } \\
\text { "60s" } \\
\mathrm{n}=80 \\
{\left[\mathrm{~N}^{*} \mathrm{~mm}^{-2}\right]}\end{array}$ & $\begin{array}{c}\text { Abhebefestig- } \\
\text { keit } \\
\text { "90s" } \\
\mathrm{n}=76 \\
{\left[\mathrm{~N}^{*} \mathrm{~mm}^{-2}\right]}\end{array}$ & $\begin{array}{c}\text { Abhebefestig- } \\
\text { keit } \\
\text { "120s" } \\
\text { n=74 } \\
{\left[\mathrm{N}^{*} \mathrm{~mm}^{-2}\right]}\end{array}$ & $\begin{array}{c}\text { Abhebefestig- } \\
\text { keit } \\
\text { "180s" } \\
n=80 \\
{\left[\mathrm{~N}^{*} \mathrm{~mm}^{-2}\right]}\end{array}$ & $\begin{array}{c}\text { Abhebefestig- } \\
\text { keit } \\
\text { "240s" } \\
\mathbf{n}=80 \\
{\left[\mathrm{~N}^{*} \mathrm{~mm}^{-2}\right]}\end{array}$ \\
\hline Presszeit $\mathbf{s}$ & 60 & 90 & 120 & 180 & 240 \\
\hline Median Z & 0,89 & 1,27 & 1,53 & 1,64 & 1,49 \\
\hline $\begin{array}{l}\text { Arithmetisches } \\
\text { Mittel } \mu\end{array}$ & 0,91 & 1,22 & 1,48 & 1,64 & 1,45 \\
\hline $\begin{array}{l}\text { Standardab- } \\
\text { weichung S }\end{array}$ & 0,23 & 0,24 & 0,26 & 0,18 & 0,28 \\
\hline Minimum & 0,44 & 0,54 & 0,87 & 1,20 & 0,81 \\
\hline Maximum & 1,55 & 1,67 & 1,88 & 2,04 & 2,07 \\
\hline
\end{tabular}

Wie aus Tabelle 4-18 zu entnehmen ist, steigt die maximale Abhebefestigkeit mit zunehmender Pressdauer stetig an, bis sie bei $240 \mathrm{~s}$ wieder abfällt (vergl. hierzu Abbildung 4-19). 


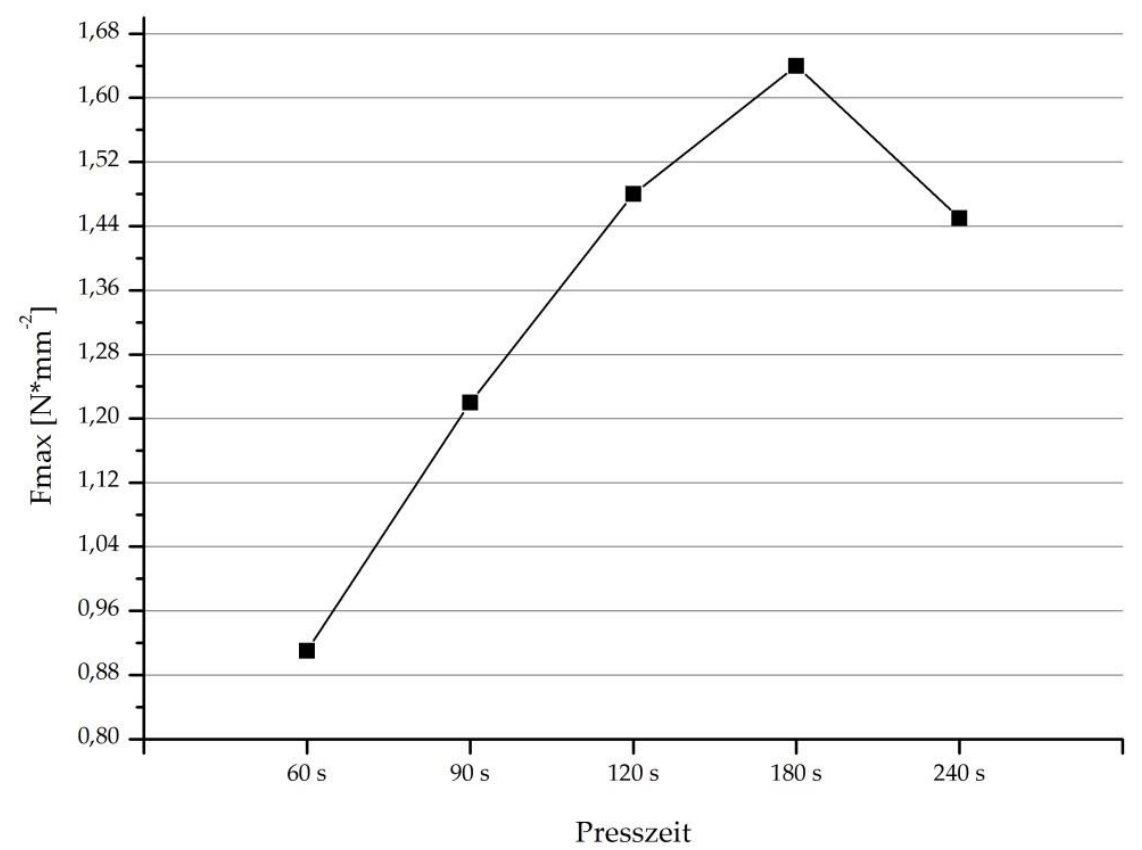

Abbildung 4-19: Entwicklung der Abhebefestigkeit von Eichenfurnier auf PVC in Relation zu den Presszeiten.

Bei einer Presszeit von $60 \mathrm{~s}$ wurde bei den Abhebefestigkeitsuntersuchungen eine maximale Bruchkraft von $\overline{\mathrm{x}}=0,91 \mathrm{~N}^{*} \mathrm{~mm}^{-2}$, bei einer errechneten Standardabweichung von $0,23 \mathrm{~N}^{*} \mathrm{~mm}^{-2}$, gemessen. Dieser Wert erhöht sich bei $90 \mathrm{~s}$ Presszeit um $0,31 \mathrm{~N}^{*} \mathrm{~mm}^{-2}$ auf $\bar{x}=1,22 \mathrm{~N}^{*} \mathrm{~mm}^{-2}\left(\mathrm{~S}=0,24 \mathrm{~N}^{*} \mathrm{~mm}^{-2}\right)$. Bei einer Presszeit von $120 \mathrm{~s}$ wird im Mittel eine maximale Bruchkraft von $\bar{x}=1,48 \mathrm{~N}^{*} \mathrm{~mm}^{-2}\left(\mathrm{~S}=0,26 \mathrm{~N}^{*} \mathrm{~mm}^{-2}\right)$ erreicht, was einen weiteren Anstieg der Abhebefestigkeit um 0,26 N*mm-2 bedeutet. Nach $180 \mathrm{~s}$ Presszeit wurde mit einem Mittelwert von $\bar{x}=1,64 \mathrm{~N}^{*} \mathrm{~mm}^{-2}$, bei einer Standardabweichung von $0,18 \mathrm{~N}^{*} \mathrm{~mm}^{-2}$, die höchste Abhebefestigkeit gemessen. Die Prüfung der Abhebefestigkeit nach $240 \mathrm{~s}$ Presszeit ergab einen mittleren Messwert von $\overline{\mathrm{x}}=1,45 \mathrm{~N}^{*} \mathrm{~mm}^{-2}$, bei einer errechneten Standardabweichung von $0,28 \mathrm{~N}^{*} \mathrm{~mm}^{-2}$. Dieser Wert liegt um $0,19 \mathrm{~N}^{*} \mathrm{~mm}^{-2}$ niedriger als bei der Messreihe $180 \mathrm{~s}$ und 0,03 $\mathrm{N}^{*} \mathrm{~mm}^{-2}$ unter dem Ergebnis der Messreihe $120 \mathrm{~s}$.

Die Extremwerte schwanken über alle Messreihen hinweg vom Minimum mit $0,44 \mathrm{~N}^{*} \mathrm{~mm}^{-2}$ bis zum Maximum von $2,07 \mathrm{~N}^{*} \mathrm{~mm}^{-2}$, was einer Differenz von $1,63 \mathrm{~N}^{*} \mathrm{~mm}^{-2}$ entspricht.

Für den Vergleich der Mittelwerte der Versuchsreihen werden folgende Hypothesen aufgestellt:

$H_{0}$ : Die jeweiligen arithmetischen Mittelwerte der $F_{\max }$ bezogen auf Presszeit, unterscheiden sich nicht. 
$H_{1}$ : Die jeweiligen arithmetischen Mittelwerte der $F_{\max }$, bezogen auf die Presszeit, unterscheiden sich signifikant.

Bei Betrachtung von Abbildung 4-20 und der Lage- und Streuparameter in Tabelle 4-18 ist davon auszugehen, dass sich die Mittelwerte der Grundgesamtheiten, sprich die Werte der Abhebefestigkeit der einzelnen Versuchsreihen, unterscheiden.

Die Versuchsreihe „120 s“ stellte sich nach Überprüfung mittels Shapiro-Wilk-Test als nicht normalverteilte Grundgesamtheit heraus. Ebenso lag im Falle der Versuchsreihe „240 s“ eine heterogene Verteilung der Varianzen vor. Dementsprechend wurde bei den Vergleichen der Mittelwerte dieser Reihen ein Kruskal-Wallis-Test durchgeführt, während bei den übrigen Untersuchungen eine ANOVA angewendet wurde.

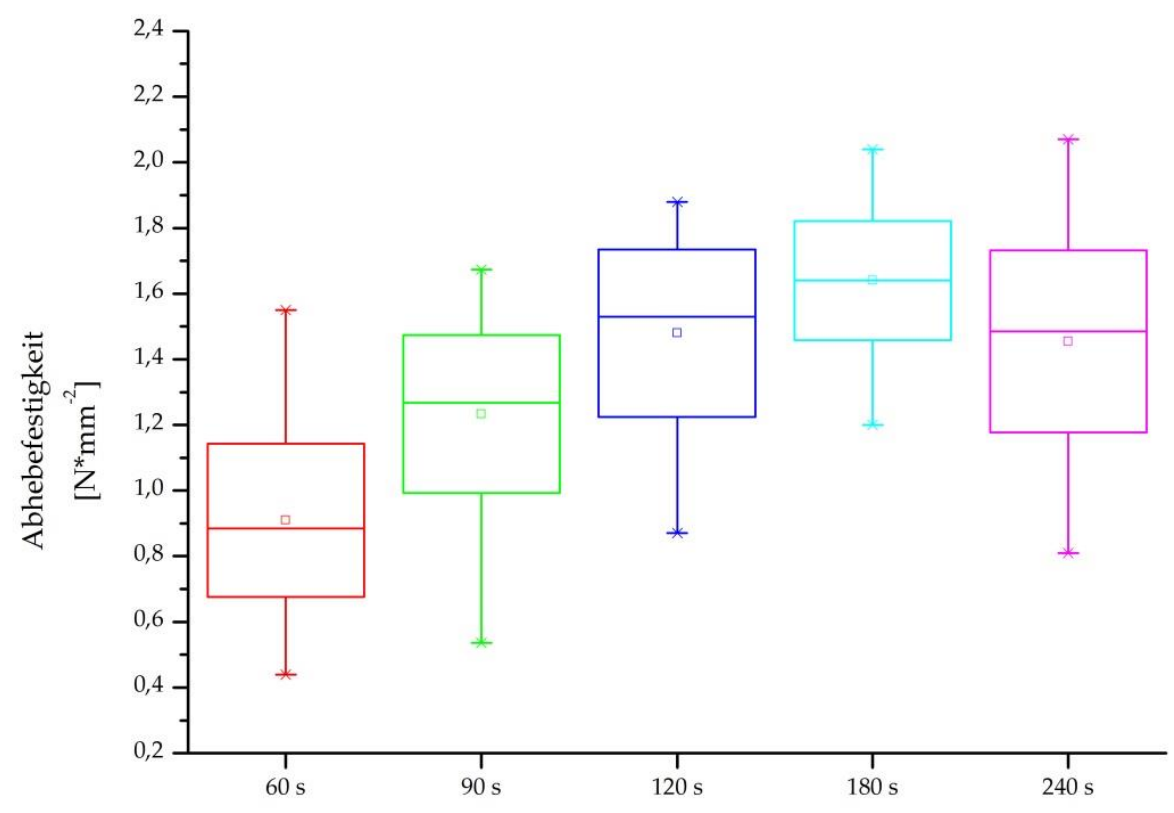

Abbildung 4-20: Vergleichende Boxplots zur Abhebefestigkeit von Eichenfurnier auf PVC, sortiert nach den jeweiligen Presszeiten.

Wie bereits durch Ableitung aus den Lage- du Streuparametern sowie der Darstellung in Abbildung 4-20 vermutet, unterscheiden sich die Mittelwerte sämtlicher Grundgesamtheiten bei einem Signifikanzniveau von 0,05 signifikant voneinander. Dies gilt auch für die um lediglich $0,03 \mathrm{~N}^{*} \mathrm{~mm}^{-2}$ differierenden Versuchsreihen "120 s" und „240 s". Somit muss für die Vergleiche sämtlicher Mittelwerte untereinander $\mathrm{H}_{1}$ angenommen und $\mathrm{H}_{0}$ verworfen werden. 


\section{Diskussion der Versuchsergebnisse}

Betrachtet man zunächst die Entwicklung der Abhebefestigkeiten von Eichenfurnier auf PVC-Folie im Gesamten so fällt auf, dass der nach DIN EN 312 geforderte Minimalwert von $0,80 \mathrm{~N}^{*} \mathrm{~mm}^{-2}$ (vergl. Kapitel 1.2) bei allen geprüften Presszeiten erreicht wird. Die Festigkeitswerte steigen zunächst mit andauernder Presszeit von im Mittel 0,91 $\mathrm{N}^{*} \mathrm{~mm}^{-2}$ bei „60 s“ bis auf 1,64 $\mathrm{N}^{*} \mathrm{~mm}^{-2}$ im Mittel bei „180 s" an um ab diesem Zeitpunkt wieder auf $\bar{x}=1,45 \mathrm{~N}^{*} \mathrm{~mm}^{-2}$ (bei „240 s“) zu fallen.

Betrachtet man nun die Messreihen im Einzelnen so fällt auf, dass sich innerhalb der Versuchsreihen "60 s", "180s" und "240s" die Mittelwerte je nach Lage in der Probeplatte signifikant voneinander unterscheiden.

Im Falle der Versuchsreihe „60 s" sind es die Lagen „oben“ und „unten“, die einen signifikanten Unterschied aufweisen. Da die mittlere maximale Bruchkraft in der Lage „oben“ 0,20 N*mm-2 niedriger liegt als in der Lage „unten“, ist ein Effekt der Struktur in der PVC-Folie in Betracht zu ziehen, da die strukturierte Seite grundsätzlich nach oben ausgerichtet wurde. Der Grund hierfür könnte in einer schlechteren Benetzung mit der Klebefolie aufgrund der kurzen Presszeit und durch die Struktur der PVCFolie vergrößerte Oberfläche zu suchen sein. Einen weiteren Hinweis darauf liefert der fehlende Unterschied zwischen den Mittelwerten der Lagen "Mitte“ und „Rand“, was grundsätzlich auf eine einheitliche Qualität der Verklebung in der Fläche schließen lässt.

Abweichend von den Ergebnissen bei einer Presszeit von „60 s“ stellt sich die Situation innerhalb der Versuchsreihe "180 s“ dar. Die Differenz zwischen den beiden Lagen „Mitte“ und „Rand“ beträgt 0,14 $\mathrm{N}^{*} \mathrm{~mm}^{-2}$. So scheint im Gegensatz zur vorherigen Versuchsreihe die Struktur der PVC-Folie keinen Einfluss auf die Qualität der Verklebung zu haben. Vielmehr entsteht der Eindruck, dass trotz der hier vorliegenden höchsten Festigkeitswerte mit $\bar{x}=1,64 \mathrm{~N}^{*} \mathrm{~mm}^{-2}$ im Rahmen der Versuchsreihen von Eichenfurnier auf PVC ein Problem in der Qualität der flächigen Verklebung vorliegt. Da für die Herstellung von Furnieren vorwiegend ältere Stämme Verwendung finden und diese einen höherer Extraktstoffgehalt sowie einen niedrigeren $\mathrm{pH}$-Wert als junge Eichen aufweisen (Roffael und Rauch 1974), könnten diese Fakten für die oben beschriebenen Differenzen verantwortlich sein. Auf den Möglichen Einfluss der Extraktstoffe und des $\mathrm{pH}$-Wertes von Eiche auf die Qualität der Verklebung des in dieser Arbeit behandelten Werkstoffes wird in Kapitel 4.4.1 noch genauer eingegangen werden.

Während die Differenz der Abhebefestigkeit zwischen den einzelnen Lagen bisher auf jeweils zwei Lageparameter begrenzt war, unterscheiden sich in der Versuchsreihe "240 s" sowohl die Mittelwerte zwischen "oben“ und „unten“ als auch zwischen "Mitte“ und „Rand“ signifikant. In Festigkeiten differieren „oben“ und „unten“ um 0,17 $\mathrm{N}^{*} \mathrm{~mm}^{-2}$, „Mitte“ und „Rand“ um 0,11 $\mathrm{N}^{*} \mathrm{~mm}^{-2}$. Somit kann festgestellt werden, 
dass sich nicht nur die Abhebefestigkeit bei längeren Presszeiten signifikant verschlechtert, sondern das somit auch die Qualität der Verklebung über die Fläche deutlich schwankt, sich also verschlechtert.

Da weder Presszeit noch Pressdruck verändert wurden, muss die Ursache für o. g. Beobachtungen konsequenterweise in der Dauer der thermischen Belastung zu suchen sein. Da laut technischem Datenblatt des Klebstoffherstellers die kritische Verarbeitungstemperatur erst ab $200{ }^{\circ} \mathrm{C}$ beginnt (Collano Adhesives AG 2011), sollte der Klebstoff als Auslöser für die nachlassenden Festigkeitseigenschaften ausgeschlossen werden können.

Somit kommen wie schon im Rahmen der Versuchsreihe "180 s" Wechselwirkungen zwischen Eichenfurnier, Klebstoff und/oder PVC-Folie in Betracht, die wie bereits erwähnt, in den folgenden Kapiteln intensiver beleuchtet werden. 


\subsubsection{Vergleiche der Abhebefestigkeit zwischen Erlenfurnier und Eichenfurnier auf PVC-Folie}

Wie in den Einzelkapiteln 4.3.1und 4.3.2 $\mathrm{zu}$ beobachten ist, scheinen sich die Abhebefestigkeiten und damit die adhäsiven Eigenschaften von Erlen- und Eichenfurnieren, die unter Verwendung von thermoplastischem Klebefilm mit PVCFolie verklebt wurden, zu unterscheiden (vergl. Abbildung 4-21). Diese Beobachtung soll im folgenden Abschnitt durch die Gegenüberstellung der Versuchsergebnisse genauer betrachtet werden. In den Beschreibungen wird zur Wahrung der Übersicht und Leserlichkeit der Materialverbund aus Erlenfurnier und PVC-Folie als Erle bezeichnet und der Verbund aus Eichenfurnier und PVC-Folie als Eiche.

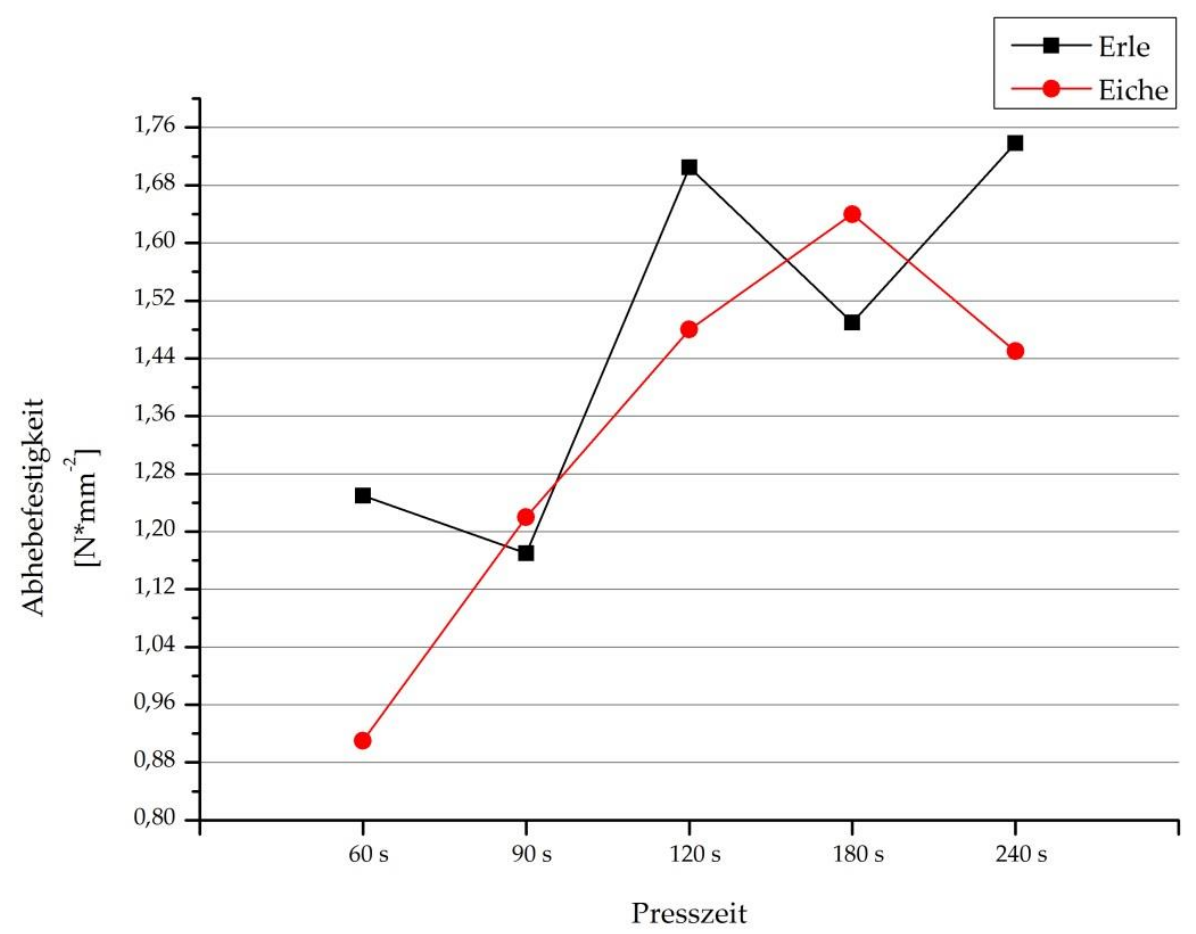

Abbildung 4-21: Entwicklung der Abhebefestigkeit von Erlen- und Eichenfurnier auf PVC-Folie, verklebt mit thermoplastischem Klebefilm, in Relation zu den Presszeiten.

Tabelle 4-19 und Tabelle 4-20 stellen noch einmal die Gesamtergebnisse der Abhebefestigkeiten von Erlen- und Eichenfurnier auf PVC-Folie (verklebt mit thermoplastischem Klebefilm) dar.

In der Versuchsreihe Erle "60 s“ wurde eine mittlere Abhebefestigkeit von 1,25 N* $\mathrm{mm}^{-2}$ $\left(\mathrm{S}=0,31 \mathrm{~N}^{*} \mathrm{~mm}^{-2}\right)$ gemessen. Bei Eiche „60 $\mathrm{s}^{\prime \prime}$ lag der Wert hingegen bei $0,91 \mathrm{~N}^{*} \mathrm{~mm}^{-2}$ $\left(\mathrm{S}=0,23 \mathrm{~N}^{*} \mathrm{~mm}^{-2}\right)$. 
Tabelle 4-19: Vergleichende Ergebnisse der Abhebefestigkeitsprüfungen von Erlenfurnier auf PVCFolie, verklebt mit thermoplastischem Klebefilm, bei verschiedenen Presszeiten.

\begin{tabular}{|l|c|c|c|c|c|}
\hline Parameter & $\begin{array}{c}\text { Abhebefestig- } \\
\text { keit } \\
\text { "60s" } \\
\mathbf{n = 5 7} \\
{\left[\mathbf{N}^{*} \mathbf{m m}^{-2}\right]}\end{array}$ & $\begin{array}{c}\text { Abhebefestig- } \\
\text { keit } \\
\text { "90s" } \\
\mathbf{n = 6 1} \\
{\left[\mathbf{N}^{*} \mathbf{m m}^{-2}\right]}\end{array}$ & $\begin{array}{c}\text { Abhebefestig- } \\
\text { keit } \\
\text { "120s" } \\
\mathbf{n = 6 8} \\
{\left[\mathbf{N}^{*} \mathbf{m m}^{-2}\right]}\end{array}$ & $\begin{array}{c}\text { Abhebefestig- } \\
\text { keit } \\
\text { "180s" } \\
\mathbf{n = 5 0} \\
{\left[\mathbf{N}^{*} \mathbf{m m}^{-2}\right]}\end{array}$ & $\begin{array}{c}\text { Abhebefestig- } \\
\text { keit } \\
\text { "240s" } \\
\mathbf{n}=73 \\
{\left[\mathbf{N}^{*} \mathbf{m m}^{-2}\right]}\end{array}$ \\
\hline Median Z & 1,24 & 1,18 & 1,65 & 1,55 & 1,82 \\
\hline $\begin{array}{l}\text { Arithmetisches } \\
\text { Mittel } \boldsymbol{\mu}\end{array}$ & 1,25 & 1,17 & 1,71 & 1,49 & 1,74 \\
\hline $\begin{array}{l}\text { Standardab- } \\
\text { weichung } \mathbf{S}\end{array}$ & 0,31 & 0,28 & 0,27 & 0,37 & 0,25 \\
\hline Minimum & 0,46 & 0,58 & 1,05 & 0,61 & 1,16 \\
\hline Maximum & 1,84 & 1,82 & 2,16 & 2,20 & 2,22 \\
\hline
\end{tabular}

Im Rahmen der Versuchsreihen "90 s“ wurde bei Eiche eine um 0,05 N*mm² höhere Abhebefestigkeit als bei Erle gemessen. Die Reihe Eiche "90 s" erreichte eine mittlere Abhebefestigkeit von 1,22 $\mathrm{N}^{*} \mathrm{~mm}^{-2}$ mit einer errechneten Standardabweichung von $0,24 \mathrm{~N}^{*} \mathrm{~mm}^{-2}$. Bei Erle „90 s“ lag der Mittelwert bei 1,17 $\mathrm{N}^{*} \mathrm{~mm}^{-2}\left(\mathrm{~S}=0,28 \mathrm{~N}^{*} \mathrm{~mm}^{-2}\right)$.

Tabelle 4-20: Vergleichende Ergebnisse der Abhebefestigkeitsprüfungen von Eichenfurnier auf PVCFolie, verklebt mit thermoplastischem Klebefilm, bei verschiedenen Presszeiten.

\begin{tabular}{|l|c|c|c|c|c|}
\hline Parameter & $\begin{array}{c}\text { Abhebefestig- } \\
\text { keit } \\
\text { "60s" } \\
\mathbf{n = 8 0} \\
{\left[\mathbf{N}^{*} \mathbf{m m}^{-2}\right]}\end{array}$ & $\begin{array}{c}\text { Abhebefestig- } \\
\text { keit } \\
\mathbf{9 0 0 s "} \\
\mathbf{n = 7 6} \\
{\left[\mathbf{N}^{*} \mathbf{m m}^{-2}\right]}\end{array}$ & $\begin{array}{c}\text { Abhebefestig- } \\
\text { keit } \\
\mathbf{1 2 0 s "} \\
\mathbf{n = 7 4} \\
{\left[\mathbf{N}^{*} \mathbf{m m}^{-2}\right]}\end{array}$ & $\begin{array}{c}\text { Abhebefestig- } \\
\text { keit } \\
\mathbf{1 8 0 s "} \\
\mathbf{n = 8 0} \\
{\left[\mathbf{N}^{*} \mathbf{m m}^{-2}\right]}\end{array}$ & $\begin{array}{c}\text { Abhebefestig- } \\
\text { keit } \\
\text { "240s" } \\
\mathbf{n = 8 0} \\
{\left[\mathbf{N}^{*} \mathbf{m m}^{-2}\right]}\end{array}$ \\
\hline Presszeit s & 60 & 90 & 120 & 180 & 240 \\
\hline Median Z & 0,89 & 1,27 & 1,53 & 1,64 & 1,49 \\
\hline $\begin{array}{l}\text { Arithmetisches } \\
\text { Mittel } \boldsymbol{\mu}\end{array}$ & 0,91 & 1,22 & 1,48 & 1,64 & 1,45 \\
\hline $\begin{array}{l}\text { Standardab- } \\
\text { weichung S }\end{array}$ & 0,23 & 0,24 & 0,26 & 0,18 & 0,28 \\
\hline Minimum & 0,44 & 0,54 & 0,87 & 1,20 & 0,81 \\
\hline Maximum & 1,55 & 1,67 & 1,88 & 2,04 & 2,07 \\
\hline
\end{tabular}

Die Messwerte der Abhebefestigkeitsversuche bei 120 s Presszeit variierten zwischen Erle und Eiche um $0,23 \mathrm{~N}^{*} \mathrm{~mm}^{-2}$. Erle „120 s“ erreichte eine mittlere Abhebefestigkeit von $1,71 \mathrm{~N}^{*} \mathrm{~mm}^{-2}$ bei einer errechneten Standardabweichung von $0,27 \mathrm{~N}^{*} \mathrm{~mm}^{-2}$ und zeigt somit deutlich höhere Abhebefestigkeiten als der Mittelwert bei Eiche „120 s" mit $1,48 \mathrm{~N}^{*} \mathrm{~mm}^{-2}\left(\mathrm{~S}=0,26 \mathrm{~N}^{*} \mathrm{~mm}^{-2}\right)$.

In der Versuchsreihe „180 s“ kehrte sich das Ergebnis der vorherigen Serie um. Bei Eiche „180 s“ konnte eine mittlere Abhebefestigkeit von 1,64 $\mathrm{N}^{*} \mathrm{~mm}^{-2}\left(\mathrm{~S}=0,18 \mathrm{~N}^{*} \mathrm{~mm}^{-2}\right)$ gemessen werden. In der Versuchsreihe Erle „180 s“ fiel die mittlere Abhebefestigkeit mit $1,49 \mathrm{~N}^{*} \mathrm{~mm}^{-2}$ und einer hohen Standardabweichung von $0,37 \mathrm{~N}^{*} \mathrm{~mm}^{-2}$ um $0,15 \mathrm{~N}^{*} \mathrm{~mm}^{-2}$ geringer aus als bei Eiche "180 s“. 
Während in der Versuchsreihe Erle "240 s" die mittlere Abhebefestigkeit im Vergleich $\mathrm{zu}, 180 \mathrm{~s}^{\prime}$ deutlich um $0,25 \mathrm{~N}^{*} \mathrm{~mm}^{-2}$ auf 1,74 $\mathrm{N}^{*} \mathrm{~mm}^{-2}\left(\mathrm{~S}=0,25 \mathrm{~N}^{*} \mathrm{~mm}^{-2}\right)$ anstieg, fiel der Wert bei Eiche „240 $\mathrm{s}^{\prime \prime}$ ähnlich deutlich um $0,19 \mathrm{~N}^{*} \mathrm{~mm}^{-2}$ auf $1,45 \mathrm{~N}^{*} \mathrm{~mm}^{-2}$ $\left(\mathrm{S}=0,28 \mathrm{~N}^{*} \mathrm{~mm}^{-2}\right) \mathrm{ab}$.

Wie schon zuvor, sollen zwei Hypothesen bzgl. der Mittelwerte der Versuchsergebnisse aufgestellt werden:

$H_{0}$ : Die jeweiligen arithmetischen Mittelwerte der $F_{\max }$, bezogen auf die Presszeit und die Furnierart, unterscheiden sich nicht.

$H_{1}$ : Die jeweiligen arithmetischen Mittelwerte der $F_{\max }$, bezogen auf die Presszeit und die Furnierart, unterscheiden sich signifikant.

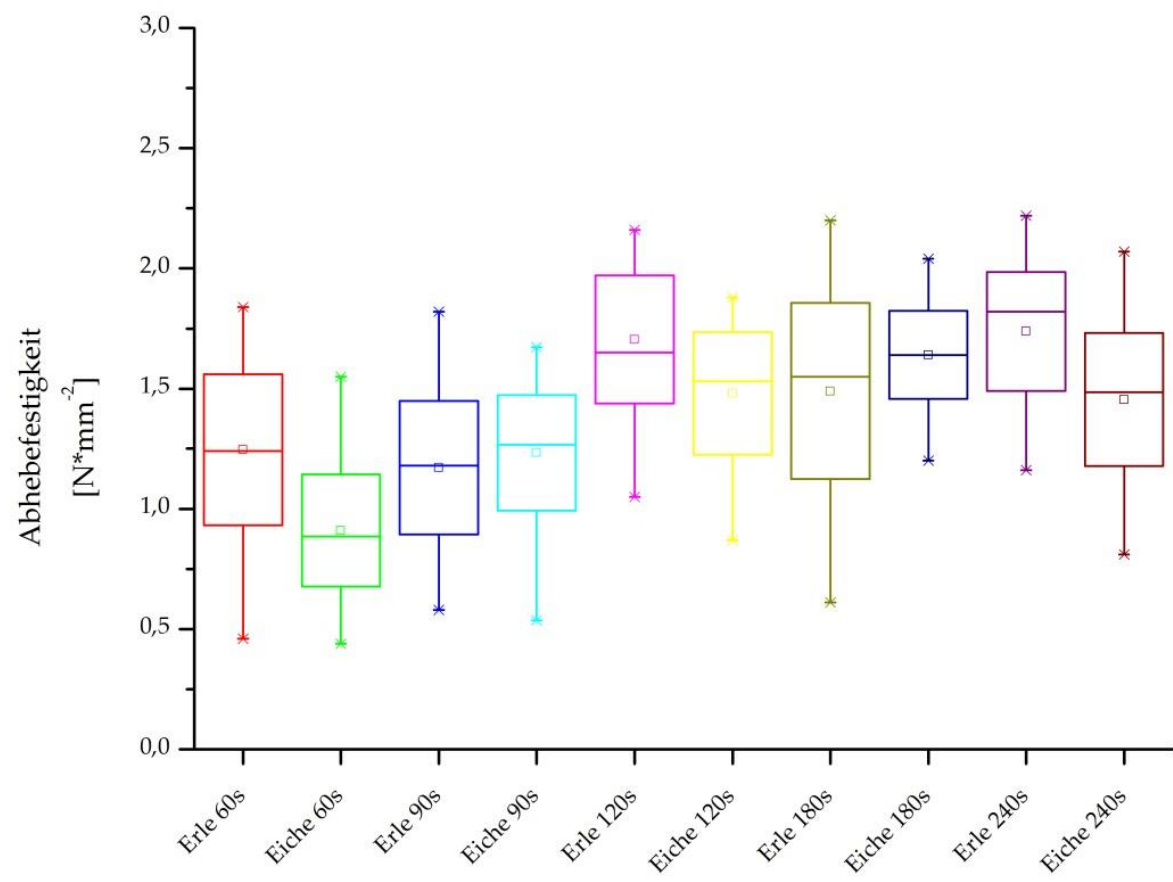

Abbildung 4-22: Boxplots zu den Abhebefestigkeitsprüfungen von Erlen- und Eichenfurnier auf PVCFolie, verklebt mit thermoplastischem Klebefilm, bei verschiedenen Presszeiten.

Da bereits in den Kapiteln 4.3.1 und 4.3.2 die Mittelwerte der Abhebefestigkeiten für die einzelnen Furnierarten in sich verglichen und diskutiert wurden, beziehen sich die folgenden Vergleiche auf die Mittelwerte der beiden Furnierarten bei gleicher Presszeit.

Wie aus Abbildung 4-22 ersichtlich, zeigt sich ein indifferentes Bild. Bei der Betrachtung der Lage- und Streuparameter kann zumindest für die Mittelwertsvergleiche der Versuchsreihen Erle "60 s" und Eiche "60 s" sowie Erle "240 s" und Eiche "240 s" mit hoher Wahrscheinlichkeit von signifikanten 
Unterschieden der Mittelwerte ausgegangen werden. Besonders Erle „60 s" und Erle „180 s“ zeigen vergleichsweise hohe Standardabweichungen.

Der statistische Vergleich der Mittelwerte von Erle "90 s" und Eiche "90 s" wurde mittels einfaktorieller ANOVA durchgeführt. Bei den weiteren Versuchsreihen lagen entweder nicht normalverteile Grundgesamtheiten vor oder es war eine Varianzheterogenität gegeben (vergl. Kapitel 4.3.1und Kapitel 4.3.2). Demzufolge wurden die Vergleiche der Mittelwerte der Serien "60 s“, „120 s“, „180 s“ und „240 s" mittels Kruskal-Wallis-Test durchgeführt.

Bei einem Signifikanzniveau von 0,05 unterschieden sich die Mittelwerte der Grundgesamtheiten der einzelnen Versuchsreihen bis auf eine Ausnahme signifikant voneinander. Lediglich bei den Mittelwerten von Erle "90 s" und Eiche "90 s" lag kein signifikanter Unterschied vor.

Somit muss $H_{1}$ für die Vergleiche der Mittelwerte von Erle "60 s" und Eiche „60 s", Erle „120 s" und Eiche „120 s", Erle „180 s" und Eiche „180 s" sowie für Erle "240 s" und Eiche "240 s" angenommen und Ho verworfen werden.

Für den Mittelwertsverlgeich von Erle "90 s" und Eiche "90 s" muss hingegen $\mathrm{H}_{0}$ angenommen und $\mathrm{H}_{1}$ verworfen werden. Die beiden Mittelwerte unterscheiden sich nicht signifikant voneinander.

\section{Diskussion der Versuchsergebnisse}

Das auffällig indifferente Verhalten der Abhebefestigkeit (siehe Abbildung 4-21) zwischen den beiden Furnierarten, insbesondere bei Erle, wurde bereits eingehende in den entsprechenden Kapiteln (vergl. Kapitel 4.3.1 und Kapitel 4.3.2) behandelt.

Da in der Versuchsreihe "90 s" kein signifikanter Unterschied zwischen Erle und Eiche festgestellt werden konnte, findet diese keinen weiteren Eingang in die Betrachtungen.

Eichenfurnier auf PVC-Folie erreichte nur in der Versuchsreihe "180 s" höhere Ergebnisse der Abhebefestigkeit als das Erlenfurnier auf PVC-Folie in der gleichen Versuchsreihe. Diese Reihe ist bei Erlenfurnier auf PVC-Folie jedoch kritisch zu betrachten, da neben der hohen Standardabweichung auch überdurchschnittlich viele Probekörper aufgrund fehlerhafter Prüfungen verworfen werden mussten (vergl. Kapitel 4.3.1).

Da diese Versuchsreihe in sich wenig konsistent erscheint, lässt sie sich dementsprechend auch nur schwierig zu einem Vergleich verwenden.

Für die Versuchsreihen „60 s“, „120s“ und "240s" lässt sich sagen, dass bei Erlenfurnier auf PVC-Folie signifikant höhere Abhebefestigkeiten gemessen wurden als bei Eichenfurnier auf PVC-Folie. Die Differenz der gemittelten Abhebefestigkeiten bei Erle fiel mindestens $0,23 \mathrm{~N}^{*} \mathrm{~mm}^{-2}\left({ }_{,} 120 \mathrm{~s}^{\prime \prime}\right)$ höher aus als bei den vergleichenden Versuchsreihen mit Eiche. 
Auch wenn sich in der schwierig zu bewertenden Serie "180 s" bei Eiche eine signifikant höhere Abhebefestigkeit messen lies, und nur in dieser Versuchsreihe, so lässt sich doch feststellen, dass die Abhebefestigkeiten von Eichenfurnier auf PVCFolie in keiner Versuchsreihe an die Höchstwerte von Erlenfurnier auf PVC-Folie heranreichen.

Dies führt zu der Folgerung, dass störende Einflussfaktoren im oder am Eichenfurnier zu geringeren Festigkeitswerten führen müssen, da die restlichen Variablen wie PVCFolie und Klebstoff den gleichen Chargen entsprachen wie bei den Vergleichsversuchen mit Erlenfurnier auf PVC-Folie.

Als Ursachen hierfür wurden zwei mögliche Faktoren in Betracht gezogen.

Da Eichen zu den ringporigen Laubhölzern zählen, weisen die aus ihr hergestellten Messerfurniere eine deutlich strukturiertere und haptisch feststellbar rauere Oberfläche auf als Furniere der zerstreutporigen Erle (Sachsse 1984). Aus diesem augenfälligen Grund wurde die Topographie der Oberfläche von Eichenfurnier als mögliche Ursache für die verringerten Abhebefestigkeiten in Betracht gezogen. Diese Hypothese wird in Kapitel 4.4.2 genauer untersucht.

Da in der Literatur vielfältig negativen Auswirkungen der Holzextraktstoffe von Eiche auf Verklebungen bekannt sind (Roffael und Rauch 1974; Roffael und Schäfer 2002; Popper 1974; Fengel und Wegener 2003; Faix 2012; Dunky und Niemz 2002; Christiansen 1991), muss die Vermutung wiederholt werden, dass die Extrakte auch hier die Verklebung negativ beeinflussen (vergl. 4.3.2).

Kapitel 4.4.1 befasst sich tiefergehend mit dieser zweiten Vermutung.

Für Erlenholz sind keine Beeinträchtigungen von Verklebungen beschrieben. Vielmehr gelten Verleimungen von Erlenholz als besonders fest und ihr Holz als chemisch nur wenig reaktiv (Grosser 2003)(vergl. 4.3.1 bis 4.3.5).

Aufgrund der schlechteren Abhebefestigkeiten von Eichenfurnieren auf PVC-Folie wurde seitens des Projektkonsortiums beschlossen, nur noch mit Erlenfurnier als Absperrfurnier $\mathrm{zu}$ arbeiten. Daher entfielen die weiteren Materialprüfungen wie Biegefestigkeit, Falzfestigkeit etc. mit Eichenfurnier. 


\subsubsection{Untersuchungen der Abhebefestigkeit von mittels PVAc- Leim verklebten Erlenfurnieren}

PVAc basierte Furnierleime sind beim Projektpartner, der Firma "Josef Wochner GmbH \&Co. KG", wesentlich für die Verarbeitung von Furnieren, da sie aufgrund ihrer stark polaren Acetatgruppe „sehr gute Haftungseigenschaften auf vielen Fügeteiloberflächen“ haben (Habenicht 2002). Nicht nur bei o. g. Projektpartner, sondern in der Holz- und Möbelindustrie generell, zählen PVAc basierte Leime zu den wichtigsten Klebstoffen (Zeppenfeld und Grunwald 2005). (Dunky 2002a) beschreibt PVAc-Leime sogar als die zweitwichtigsten Klebstoffe nach Harnstoff-FormaldehydHarzen in der Möbelindustrie.

Da Absperr- und Deckfurniere des Werkstoffs mittels PVAc basierten Leims, namentlich „Kleiberit 322“, verklebt wurden, erfolgte auch für diesen Verbund eine Abhebefestigkeitsprüfung um die Werte vergleichend bewerten zu können.

Tabelle 4-21 beschreibt die Herstellungsparameter der mittels PVAc-Leim verklebten Erlenfurnierplatten. Die aufgetragene Klebstoffmenge von $175 \mathrm{~g}^{*} \mathrm{~m}^{-2}$ wurde als Mittelwert der einzelnen Messungen dargestellt, da geringe Schwankungen beim händischen Auftrag auftreten. Im Gegensatz zur Heißverpressung des Verbundes aus Erlenfurnier auf PVC-Folie, wird der hier betrachtete Materialverbund bei Raumtemperatur (ca. $20^{\circ} \mathrm{C}$ während der Plattenherstellung) und bei höherem Pressdruck verpresst. Die Verweildauer in der Presse betrug 1.800 s (30 min).

Tabelle 4-21: Herstellungsparameter des untersuchten Furnierplatten aus Erlenfurnier auf Erlenfurnier verklebt mittels „Kleiberit 322“.

\begin{tabular}{|l|l|}
\hline Material & $\begin{array}{l}\text { Messerfurniere Erle auf Messerfurnier } \\
\text { Erle }\end{array}$ \\
\hline Plattenformat & $750 \mathrm{~mm} \times 300 \mathrm{~mm}$ \\
\hline Furnierdicke & $\overline{\mathrm{x}}=0,54 \mathrm{~mm}$ \\
\hline Klebstoff & Kleiberit 322 \\
\hline Klebstoffmenge & $\overline{\mathrm{x}}=175 \mathrm{~g}^{*} \mathrm{~m}^{-2}$ \\
\hline Presszeit & $1.800 \mathrm{~s}$ \\
\hline Presstemperatur & $\mathrm{ca} .20^{\circ} \mathrm{C}$ \\
\hline Pressdruck & $24,5 \mathrm{~N}^{*} \mathrm{~cm}^{-2}$ \\
\hline
\end{tabular}

Die Schließzeit der Presse ist aufgrund der ohnehin langen Presszeit irrelevant.

Wie in den vorangegangenen Kapiteln werden auch hier zunächst die Ergebnisse der Abhebefestigkeitsprüfung in Summe und im Anschluss in Bezug auf ihre Lage in den Probeplatten betrachtet. Im Anschluss werden zwei Hypothesen, in Bezug auf die Mittelwerte, aufgestellt 
Tabelle 4-22 stellt die Versuchsergebnisse der Abhebefestigkeitsprüfungen von Erlenfurnier auf Erlenfurnier verklebt mit „Kleiberit 322“ dar.

Tabelle 4-22: Ergebnisse der Abhebefestigkeitsprüfung von Erlenfurnier auf Erlenfurnier verklebt mit „Kleiberit 322“ bei 120 Sekunden Presszeit, differenziert nach dem Gesamtergebnis und den jeweiligen Lagen der Probekörper in den Probeplatten.

\begin{tabular}{|l|c|c|c|}
\hline Parameter & $\begin{array}{c}\text { Abhebefestigkeit } \\
\text { "gesamt" } \\
\mathbf{n = 4 4} \\
{\left[\mathbf{N}^{*} \mathbf{m m}^{-\mathbf{2}]}\right.}\end{array}$ & $\begin{array}{c}\text { Abhebefestigkeit } \\
\text { "Mitte" } \\
\mathbf{n = 2 3} \\
{\left[\mathbf{N}^{*} \mathbf{m m}^{-2}\right]}\end{array}$ & $\begin{array}{c}\text { Abhebefestigkeit } \\
\text { "Rand" } \\
\mathbf{n = 2 1} \\
{\left[\mathbf{N}^{*} \mathbf{m m}^{-2}\right]}\end{array}$ \\
\hline Presszeit s & 120 & 120 & 120 \\
\hline Median Z & 2,66 & 2,64 & 2,80 \\
\hline $\begin{array}{l}\text { Arithmetisches } \\
\text { Mittel } \boldsymbol{\mu}\end{array}$ & 2,67 & 2,63 & 2,71 \\
\hline $\begin{array}{l}\text { Standardab- } \\
\text { weichung S }\end{array}$ & 0,32 & 0,27 & 0,34 \\
\hline Minimum & 2,00 & 2,13 & 2,00 \\
\hline Maximum & 3,41 & 3,13 & 3,41 \\
\hline
\end{tabular}

Das arithmetische Mittel der Abhebefestigkeit des Verbundes beträgt 2,67 $\mathrm{N}^{*} \mathrm{~mm}^{-2}$ bei einer errechneten Standardabweichung von $0,31 \mathrm{~N}^{*} \mathrm{~mm}^{-2}$. Zwischen den Extrema liegt bei einem Minimum von 2,00 N*mm-2 und einem Maximum von 3,41 $\mathrm{N}^{*} \mathrm{~mm}^{-2}$ eine Differenz von $1,41 \mathrm{~N}^{*} \mathrm{~mm}^{-2}$.

Die Festigkeitswerte der Lagen „Mitte" und "Rand“ unterscheiden sich um $0,08 \mathrm{~N}^{*} \mathrm{~mm}^{-2}$, wobei die Abhebefestigkeit der Lage „Mitte“ 2,63 N* $\mathrm{mm}^{-2}\left(\mathrm{~S}=0,31 \mathrm{~N}^{*} \mathrm{~mm}\right.$ $\left.{ }^{2}\right)$ und die der Lage „Rand“ $2,71 \mathrm{~N}^{*} \mathrm{~mm}^{-2}\left(\mathrm{~S}=0,34 \mathrm{~N}^{*} \mathrm{~mm}^{-2}\right)$ beträgt.

In der Gesamtbetrachtung wurde eine sehr hohe mittlere Abhebefestigkeit von 2,67 $\mathrm{N}^{*} \mathrm{~mm}^{-2}$ gemessen. Die Standardabweichung beträgt $0,31 \mathrm{~N}^{*} \mathrm{~mm}^{-2}$.

Für den Vergleich der Mittelwerte zwischen „Rand“ und „Mitte“ werden folgende Hypothesen aufgestellt:

$H_{0}$ : Die jeweiligen arithmetischen Mittelwerte der $F_{\max }$, bezogen auf die Lage der Probekörper in der Prüfplatte, unterscheiden sich nicht.

$H_{1}$ : Die jeweiligen arithmetischen Mittelwerte der $F_{\max }$, bezogen auf die Lage der Probekörper in der Prüfplatte, unterscheiden sich signifikant.

Betrachtet man die Lage- und Streuparameter sowie Abbildung 4-23 so muss man davon ausgehen, dass das sich die Mittelwerte der Lagen „Mitte“ und „Rand“ nicht unterscheiden. 


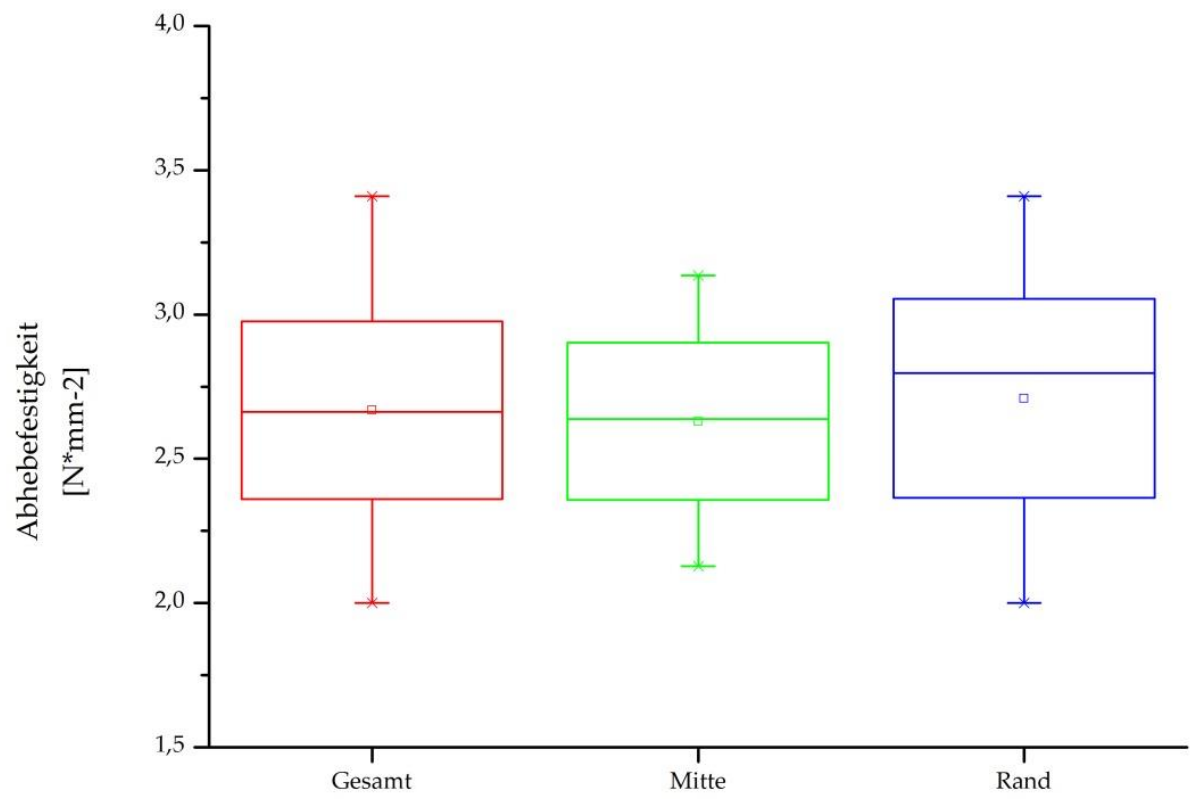

Abbildung 4-23: Boxplots zur Abhebefestigkeit von Erlenfurnier auf Erlenfurnier verklebt mit „Kleiberit 322" bei 120 Sekunden Presszeit, sortiert nach Gesamtergebnis sowie der Probenlage innerhalb der Platten.

Die Überprüfung der Hypothesen mittels ANOVA bei einem Signifikanzniveau von 0,05 bestätigt diese Annahme. Die Mittelwerte der Versuchsreihen „Mitte“ und „Rand“ unterscheiden sich nicht signifikant. Daher muss $\mathrm{H}_{0}$ angenommen und $\mathrm{H}_{1}$ verworfen werden.

\section{Diskussion der Ergebnisse}

Wie schon erwartet liefert das Ergebnisse der Abhebefestigkeit einen sehr hohen Wert, der mit einer Differenz von 0,93 $\mathrm{N}^{*} \mathrm{~mm}^{-2}$ deutlich über dem höchsten Festigkeitswert der Versuchsreihe von Erle auf PVC $\bar{x}=1,74 \mathrm{~N}^{*} \mathrm{~mm}^{-2}$ (240 s) liegt.

Da hier jedoch zwei identische Materialien mit einem speziell hierfür entworfenen Klebstoff, „Kleiberit 322“ ist ein Furnierleim (vergl. Kapitel 3.1.3.1), verbunden wurden, überrascht dieses Ergebnis nicht. Zudem werden Leimverbindungen von Erlenholz als besonders fest beschrieben (Grosser 2003).

Die hohe Qualität der Verklebung über die Fläche spiegelt sich auch in den um lediglich $0,08 \mathrm{~N}^{*} \mathrm{~mm}^{-2}$ und nicht signifikant differierenden Werten zwischen den Lagen „Mitte“ und „Rand“ wieder.

Abgeleitet aus den hohen Werten der Abhebefestigkeit aus Kapitel - 49 -4.3.1 und den noch deutlich höheren Messergebnissen der oben beschriebenen Versuchsreihe, kann man den Verbundwerkstoff bezogen auf die Qualität der Verklebungen als sehr gut 
bezeichnen. Als Referenz dienen die Normwerte der Abhebefestigkeit von Spanplatten, die im Baubereich eingesetzt werden und somit deutlich höhere Belastungen aushalten müssen als der hier entwickelte Werkstoff. Die Anforderungen an die Abhebefestigkeiten dieser Spanplatten liegt nach Niemz und Sonderegger (2017) zwischen $0,80 \mathrm{~N}^{*} \mathrm{~mm}^{-2}$ und $1,60 \mathrm{~N}^{*} \mathrm{~mm}^{-2}$.

Da im Rahmen dieser Arbeit noch eine weitere Variante zur Verklebung von Erlenfurnier mit Erlenfurnier überprüft wurde, folgt eine vergleichende Diskussion in Kapitel 4.3.5.

\subsubsection{Untersuchungen der Abhebefestigkeit von mittels thermoplastischem Klebefilm verklebten Erlenfurnieren}

Die folgenden Untersuchungen befassen sich mit der Abhebefestigkeit von Erlenfurnieren, die mit thermoplastischem Klebefilm verklebt wurden.

Im bisher beschriebenen Herstellungsverfahren des Verbundwerkstoffes geschah dieses mittels zweier verschiedener Prozesse. Zunächst erfolgt die Verklebung des Absperrfurniers auf PVC-Folie mit thermoplastischem Klebefilm durch Heißverpressung. Im zweiten Produktionsschritt werden die Absperrfurniere mittels PVAc-Leim und Kaltverpressung mit ihrem Deckfurnier, also der sichtbaren Materialschicht, verklebt.

Der Vorteil des Verfahrens liegt darin, dass sämtliche beim Industriepartner verwendeten Furniere mittels PVAc-Leims problemlos mit dem Absperrfurnier aus Erle verklebt werden können und ein hoher Erfahrungswert im Umgang mit diesen Komponenten bei den Mitarbeitenden des Projektpartners vorhanden ist.

Der Nachteil liegt im vergleichsweisen hohen Zeitaufwand. Der Materialverbund muss zwei Mal gepresst werden und mindestens $30 \mathrm{~min}$ in der Furnierpresse verbleiben, wenn dieser Pressvorgang nicht erneut unter erhöhter Presstemperatur geschieht. Eine erneute temperierte Verpressung war nicht erwünscht, um einen möglichen Temperatureinfluss auf den ersten Materialverbund aus PVC-Folie und Erlenfurnier zu vermeiden.

Ein zusätzlicher Nachteil des zweistufigen Herstellungsprozesses entsteht durch einen weiteren erhöhten zeitlichen Aufwand aufgrund der anfallenden Reinigung von verwendeten Gerätschaften.

Daher wurde überprüft, ob der Herstellungsprozess deutlich verkürzt werden kann, indem auch die beiden Furnierlagen mittels des oben erwähnten Klebfilms mit ausreichender Festigkeit verklebt werden können. 
$\mathrm{Zu}$ diesem Zweck erfolgte eine Verklebung von Erlenfurnier auf Erlenfurnier mittels des Klebstoffes "Collano 46.002" (100 g* $\left.\mathrm{m}^{-2}\right)$ bei der bereits ermittelten günstigsten Presszeit von $120 \mathrm{~s}$ und einem Pressdruck von 13,96 $\mathrm{N}^{*} \mathrm{~cm}^{-2}$. Tabelle 4-23 gibt einen Überblick zu den Herstellungsparametern.

Tabelle 4-23: Herstellungsparameter der untersuchten Furnierplatten aus Erlenfurnier auf Erlenfurnier verklebt mittels "Collano 46.002".

\begin{tabular}{|l|l|}
\hline Material & $\begin{array}{l}\text { Messerfurniere Erle auf Messerfurnier } \\
\text { Erle }\end{array}$ \\
\hline Plattenformat & $750 \mathrm{~mm} \times 300 \mathrm{~mm}$ \\
\hline Furnierdicke & $\overline{\mathrm{x}}=0,54 \mathrm{~mm}$ \\
\hline Klebstoff & Collano $46.002 / 100 \mathrm{~g}^{*} \mathrm{~m}^{-2}$ \\
\hline Presszeit & $120 \mathrm{~s}$ \\
\hline Presstemperatur & $120^{\circ} \mathrm{C}^{*}{ }^{-2}$ \\
\hline Pressdruck & $13,96 \mathrm{~N}^{*} \mathrm{Cm}^{-2}$ \\
\hline Schließzeit Presse & $\overline{\mathrm{x}}=8,83 \mathrm{~s}$ \\
\hline
\end{tabular}

Die Qualität der Verklebung wurde wie schon in Kapitel 4.3.1 erläutert anhand von Abhebefestigkeitsprüfungen untersucht.

Die Auswertung der Versuchsergebnisse erfolgt nach demselben Schema der vorangegangenen Kapitel. Zunächst werden die Mittelwerte der Abhebefestigkeit in den verschiedenen Plattenlagen miteinander verglichen. Im Anschluss erfolgt eine Bewertung der Ergebnisse für die gesamte Platte. Wie schon zuvor beschreiben die verwendeten Namen der Versuchsreihen „Mitte“ und „Rand“ die Entnahmestelle aus den Probeplatten, also Mittel- oder Randlage. Die Lagen „oben“ und „unten“ entfallen, da bei dieser Versuchsreihe keine Mittelschicht vorhanden ist. Durch diesen Umstand bedingt, fällt auch der Stichprobenumfang geringer aus. Als "gesamt" werden sämtliche Messwerte, lageunabhängig, tituliert.

\section{Abhebefestigkeit von Erlenfurnier auf Erlenfurnier bei Verklebung mittels thermoplastischen Klebefilms}

Wie aus Tabelle 4-24 ersichtlich, wurde bei der Herstellung des Verbundes aus Erlenfurnier auf Erlenfurnier mittels Verklebung durch den Klebstoff "Collano 46.002" bei einer Presszeit von $120 \mathrm{~s}$ eine mittlere Abhebefestigkeit von $\bar{x}=1,97 \mathrm{~N}^{*} \mathrm{~mm}^{-2}$ gemessen. Die Standardabweichung beträgt $0,18 \mathrm{~N}^{*} \mathrm{~mm}^{-2}$. Die Abhebefestigkeit der Lage „Mitte“ beläuft sich auf $\bar{x}=1,91 \mathrm{~N}^{*} \mathrm{~mm}^{-2}$ bei einer Standardabweichung von $0,16 \mathrm{~N}^{*} \mathrm{~mm}^{-2}$. Die Festigkeitswerte der Lage "Rand“ wurden mit 2,02 N*mm-2 gemessen. Die Standardabweichung beträgt $0,19 \mathrm{~N}^{*} \mathrm{~mm}^{-2}$. Somit differieren die Abhebefestigkeiten der Lagen "Mitte“ und "Rand“ um 0,11 N*mm-, was einer relativen Differenz von 5,45\% entspricht. Die Extrema unterscheiden sich mit einem Minimum von $1,52 \mathrm{~N}^{*} \mathrm{~mm}^{-2}$ und einem Maximum von $2,44 \mathrm{~N}^{*} \mathrm{~mm}^{-2} \mathrm{um} 0,92 \mathrm{~N}^{*} \mathrm{~mm}^{-2}$. 
Tabelle 4-24: Ergebnisse der Abhebefestigkeitsprüfung von Erlenfurnier auf Erlenfurnier verklebt mit „Collano 46.002“ bei 120 Sekunden Presszeit, differenziert nach dem Gesamtergebnis und den jeweiligen Lagen der Probekörper in den Probeplatten.

\begin{tabular}{|l|c|c|c|}
\hline Parameter & $\begin{array}{c}\text { Abhebefestigkeit } \\
\text { "gesamt" } \\
\mathbf{n = 3 9} \\
{\left[\mathbf{N}^{*} \mathbf{m m}^{-\mathbf{2}]}\right.}\end{array}$ & $\begin{array}{c}\text { Abhebefestigkeit } \\
\text { "Mitte" } \\
\mathbf{n = 1 9} \\
{\left[\mathbf{N}^{*} \mathbf{m m}^{-2}\right]}\end{array}$ & $\begin{array}{c}\text { Abhebefestigkeit } \\
\text { "Rand" } \\
\mathbf{n = 2 0} \\
{\left[\mathbf{N}^{*} \mathbf{m m}^{-2}\right]}\end{array}$ \\
\hline Presszeit s & 120 & 120 & 120 \\
\hline Median Z & 1,99 & 1,90 & 2,03 \\
\hline $\begin{array}{l}\text { Arithmetisches } \\
\text { Mittel } \boldsymbol{\mu}\end{array}$ & 1,97 & 1,91 & 2,02 \\
\hline $\begin{array}{l}\text { Standardab- } \\
\text { weichung S }\end{array}$ & 0,18 & 0,16 & 0,19 \\
\hline Minimum & 1,52 & 1,56 & 1,52 \\
\hline Maximum & 2,44 & 2,23 & 2,44 \\
\hline
\end{tabular}

Wie in den vorangegangenen Kapiteln werden auch hier folgende zwei Hypothesen aufgestellt:

$H_{0}$ : Die jeweiligen arithmetischen Mittelwerte der $F_{\max }$, bezogen auf die Lage der Probekörper in der Prüfplatte, unterscheiden sich nicht.

$H_{1}$ : Die jeweiligen arithmetischen Mittelwerte der $F_{\max }$, bezogen auf die Lage der Probekörper in der Prüfplatte, unterscheiden sich signifikant.

Aufgrund der relativ geringen Differenz zwischen den Mittelwerten und den vergleichsweise niedrigen Standardabweichungen sowie bei Betrachtung der Darstellung der Lage- und Streuparameter in Abbildung 4-24 scheinen sich die Mittelwerte der beiden Grundgesamtheiten nicht signifikant zu unterscheiden. 


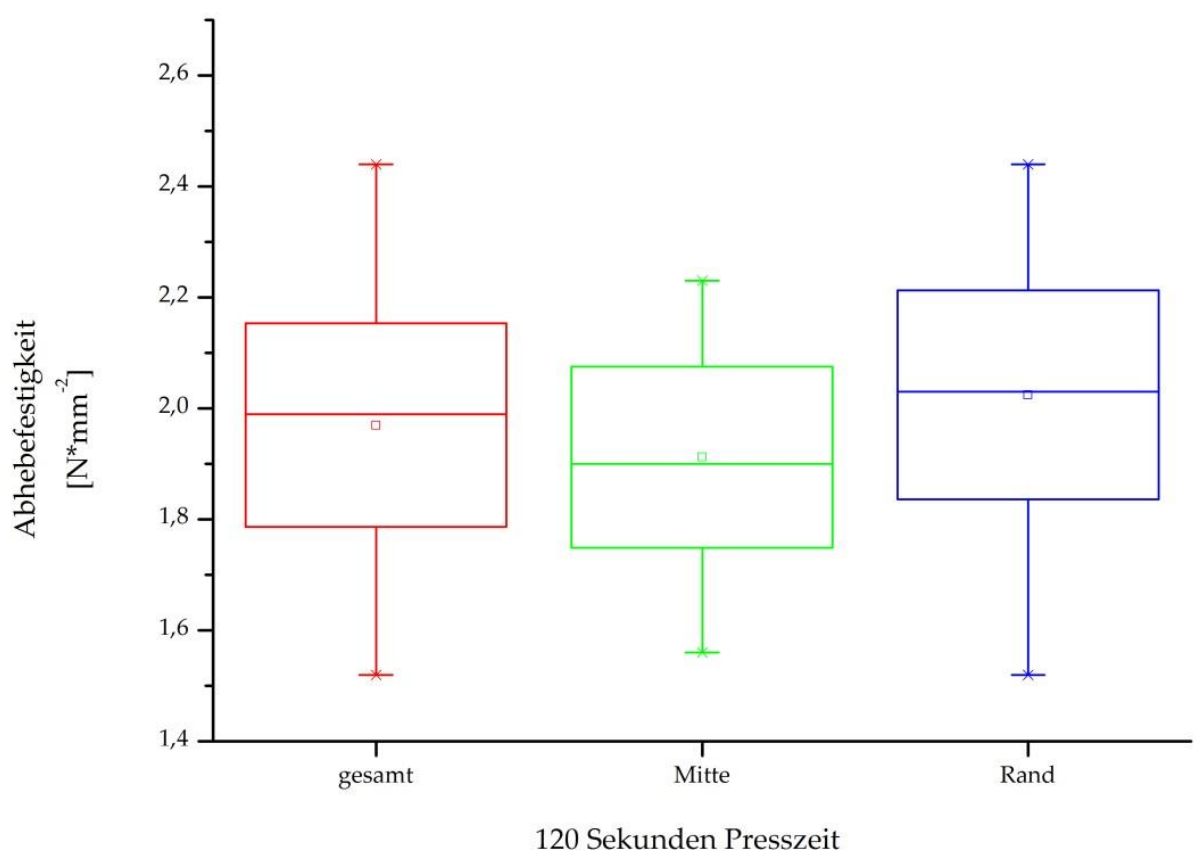

\begin{abstract}
Abbildung 4-24: Boxplots zur Abhebefestigkeit von Erlenfurnier auf Erlenfurnier verklebt mit "Collano 46.002" bei 120 Sekunden Presszeit, sortiert nach Gesamtergebnis sowie der Probenlage innerhalb der Platten.
\end{abstract}

Der Vergleich der Mittelwerte anhand einer ANOVA bei einem Signifikanzniveau von 0,05 bestätigt diese Annahme. Die Mittelwerte von „Mitte“ und „Rand“ unterscheiden sich nicht signifikant. Somit muss $\mathrm{H}_{0}$ angenommen und $\mathrm{H}_{1}$ verworfen werden.

\title{
Diskussion der Versuchsergebnisse
}

Die Versuchsergebnisse der Abhebefestigkeit zeigen eindeutig, dass sich der thermoplastische Klebefilm „Collano 46.002" dazu eignet, den Verbundwerkstoff aus Erlenfurnier und PVC-Folie in einem einzigen Pressvorgang herzustellen und auf einen PVAc basierten Klebstoff zu verzichten. Die gemessene Abhebefestigkeit liegt mit $\bar{x}=1,97 \mathrm{~N}^{*} \mathrm{~mm}^{-2}$ nicht nur weit über den ursprünglich geforderten $0,80 \mathrm{~N}^{*} \mathrm{~mm}^{-2}$ sondern auch deutlich über der von Erlenfurnier auf PVC mit $\bar{x}=1,71 \mathrm{~N}^{*} \mathrm{~mm}^{-2}$. Der Vergleich der beiden Mittelwerte mittels Kruskal-Wallis-Tests bestätigt eine signifikant (Signifikanzniveau 0,05) höhere Abhebefestigkeit bei mittels "Collano 46.022" verklebten Erlenfurnieren.

Der niedrigste gemessene Einzelwert dieser Versuchsreihe $\left(1,52 \mathrm{~N}^{*} \mathrm{~mm}^{-2}\right)$ übertrifft sogar die mittleren Abhebefestigkeiten der Versuchsreihen "60 $\mathrm{s}^{\prime \prime}$ mit 1,25 N*mm-2, „90 s“ mit 1,18 $\mathrm{N}^{*} \mathrm{~mm}^{-2}$ und „180 s“ mit 1,49 $\mathrm{N}^{*} \mathrm{~mm}^{-2}$ der Serie Erlenfurnier auf PVC (vergl. Kapitel 4.3.1.).

Dies spricht für die besonders gute Festigkeit des mittels „Collano 46.002“ verklebten Verbundes von Erlenfurnier auf Erlenfurnier. 
Da sich die Mittelwerte der Abhebefestigkeit zwischen den Lagen „Mitte“ und „Rand“ nicht signifikant unterscheiden, kann zudem eine sehr gute Qualität der Verklebung über die Fläche bestätigt werden.

Da die Presszeit von 120 s als ideal für das Erreichen einer hohen Abhebefestigkeit bei der Verarbeitung von "Collano 46.002" ermittelt wurde, muss im Folgenden eine Presszeit für den 5-schichtigen Gesamtverbund eruiert werden.

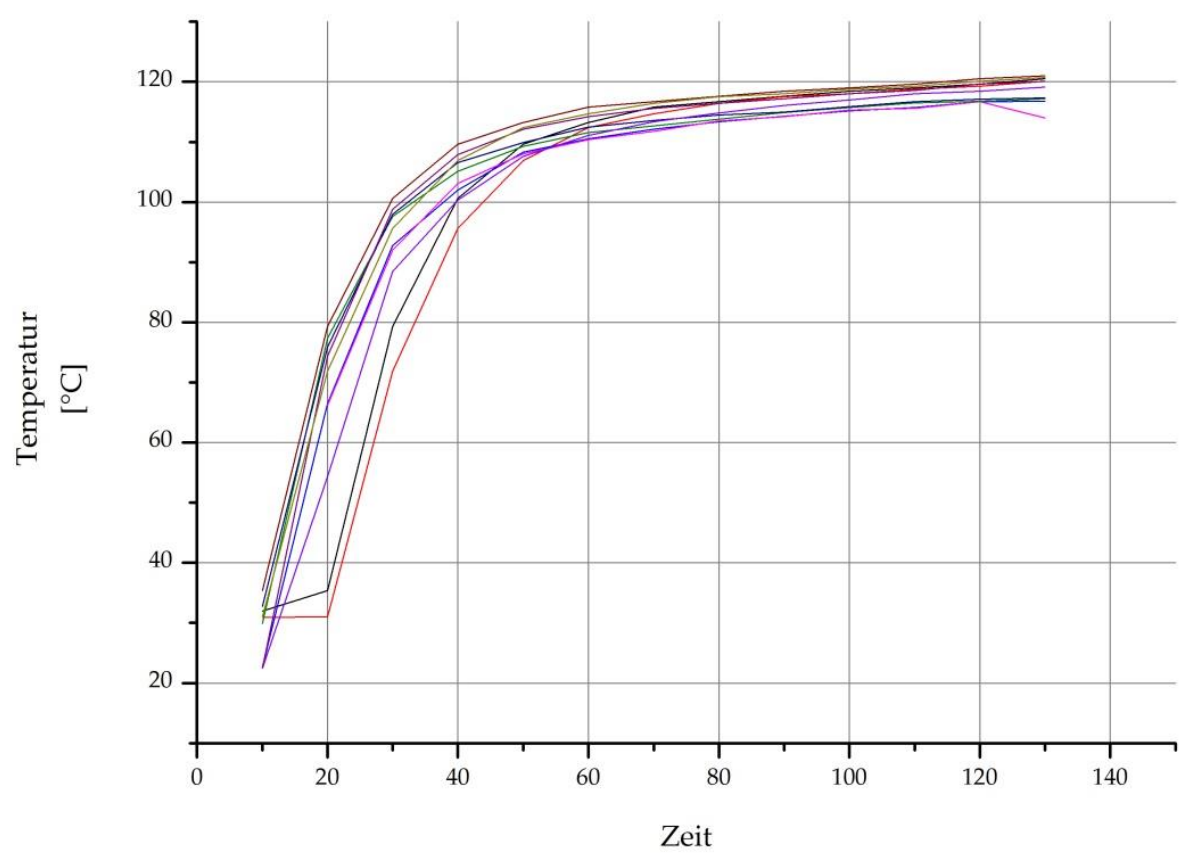

[s]

Abbildung 4-25: Temperaturverlauf über die Presszeit bei Erlenfurnier auf PVC, gemessen in den Klebefugen zwischen Furnier und PVC auf der Unter- und Oberseite.

Abbildung 4-25 zeigt den gemessenen Temperaturverlauf in den Klebefugen während des Pressvorgangs. Zur Messung der Temperatur wurde sowohl auf der Unter- als auch auf der Oberseite der Mittelschicht ein Temperaturfühler in die Klebefugen zwischen Furnier und PVC platziert.

Da laut technischem Datenblatt des Klebefilms "Collano 46.002" die minimale Klebefugentemperatur $90^{\circ} \mathrm{C}$ beträgt (Collano Adhesives AG 2011), kann man der Abbildung 4-25, unter Berücksichtigung der Schließ- und Öffnungszeit der Presse von knapp 10 s, entnehmen, dass sich der Klebstoff während des Pressvorgangs ca. 110 s in reaktiver Phase befindet.

Die Durchwärmung des Furnierblatts auf die klebstoffrelevante Minimaltemperatur ist demgemäß nach maximal $10 \mathrm{~s}$ abgeschlossen.

Da der Verbundwerkstoff beim Pressvorgang symmetrisch erhitzt wird, reicht nach o. g. Bedingungen eine Nettopresszeit von 130 s, um den gesamten Verbundwerkstoff 
mit höchstmöglicher Abhebefestigkeit bei gleichzeitig möglichst kurzer Presszeit herzustellen.

Die Herstellung des Verbundwerkstoffes in nur einem Pressvorgang würde mindestens 30 min Produktionszeit pro Platte sparen, die Rüst- und Reinigungszeiten für Werkzeuge und Maschinen noch nicht eingerechnet. Bereits nach wenigen Minuten $(<5 \mathrm{~min})$ außerhalb der Presse kann das Halbzeug weiterverarbeitet werden. Eine kurze Zwischenlagerung des noch heißen Werkstoffes unter Pressblechen (Raumtemperatur) wirkt sich positiv aus.

Aus diesen Gründen ist die Werkstoffherstellung in einem s g. One-Shot-Prozess zu empfehlen. Vorrausetzung ist jedoch, dass keine negativen Wechselwirkungen zwischen Deckfurnier und Klebstoff entstehen. Da der Projektpartner den Werkstoff besonders im Hochpreissegment einsetzten möchte und in diesem Zusammenhang häufig Furniere aus Tropenhölzern eingesetzt werden, müssen diese Materialkombinationen ggf. erneut auf ihre Eigenschaften überprüft werden.

Als im Herstellungsprozess erprobte und zuverlässige Variante der Verklebung von Furnieren untereinander gilt die in Kapitel 4.3.2 durchgeführte Version der Verleimung mittels PVAc-Klebstoffs. 


\subsubsection{Vergleichende Diskussion der Erlenfurnierverklebung unter Verwendung von PVAc-Leim und thermoplastischem} Klebefilm

Tabelle 4-25 gibt einen Überblick über die Ergebnisse der Abhebefestigkeitsprüfung der beiden Versuchsreihen "PVAc" und „Klebefilm". „PVAc" beschreibt die Verklebung von Erlenfurnier mit Erlenfurnier durch den Furnierleim „Kleiberit 322“. Als „Klebefilm" wird die Versuchsreihe zur Abhebefestigkeit von mittels "Collano 46.002" verklebten Erlenfurnieren bezeichnet.

Tabelle 4-25: Vergleichende Ergebnisse der Abhebefestigkeitsprüfung von Erlenfurnier auf Erlenfurnier verklebt mit „PVAC“ und „Klebefilm“.

\begin{tabular}{|l|c|c|}
\hline Parameter & $\begin{array}{c}\text { Abhebefestigkeit } \\
\text { "PVAc" } \\
\mathbf{n = 4 4} \\
{\left[\mathbf{N}^{*} \mathbf{m m}^{-2}\right]}\end{array}$ & $\begin{array}{c}\text { Abhebefestigkeit } \\
\text { "Klebefilm" } \\
\mathbf{n = 3 9} \\
{\left[\mathbf{N}^{*} \mathbf{m m}^{-2}\right]}\end{array}$ \\
\hline Presszeit s & 120 & 120 \\
\hline Median $\mathbf{Z}$ & 2,66 & 1,99 \\
\hline Arithmetisches Mittel $\boldsymbol{\mu}$ & 2,67 & 1,97 \\
\hline Standardabweichung $\mathbf{S}$ & 0,31 & 0,18 \\
\hline Minimum & 2,00 & 1,52 \\
\hline Maximum & 3,41 & 2,44 \\
\hline
\end{tabular}

Die ermittelten Abhebefestigkeiten zwischen „PVAc“ und „Klebefilm“ unterscheidet sich um $0,70 \mathrm{~N}^{*} \mathrm{~mm}^{-2}$, wobei die höhere Festigkeit mit $\bar{x}=2,67 \mathrm{~N}^{*} \mathrm{~mm}^{-2}$ bei der Versuchsreihe „PVAc" erreicht wird. Beide Versuchsreihen zeigen nur geringe Standardabweichungen. Dies lässt auf sehr valide Versuchsergebnisse aufgrund geringer Schwankungen schließen.

Ein statistischer Vergleich der beiden Mittelwerte mittels Kruskal-Wallis-Test (Signifikanzniveau 0,05) bestätigt einen signifikanten Unterschied der Grundgesamtheiten.

Wie in den Kapitel 4.3.2 und 4.3.5 bereits erwähnt, eigenen sich beide Klebstoffe hervorragend für die Verklebung der beiden Furnierschichten aus Erle miteinander.

Die Josef Wochner GmbH \& Co. KG muss die nun folgenden Vor- und Nachteile der beiden Klebstoffsysteme gegeneinander abwiegen.

Die Verklebung mittels PVAc-Leims bietet die Vorteile einer signifikant höheren Festigkeit und fugenfüllender Eigenschaften aufgrund seines Festkörpergehaltes von 50 \%-60 \% (Habenicht 2002; Dunky 2002a). Die Anwendungsmöglichkeit mit den meisten handelsüblichen Furnierarten ist gegeben und der Umgang mit diesem 
Klebstoff ist den meisten Handwerkern hinreichend bekannt, da dieses Klebstoffsystem zu einem der meistgenutzten in der Holz- und Möbelindustrie zählt (Zeppenfeld und Grunwald 2005).

Im Vergleich zum thermoplastischen Klebefilm kommen aber gleichzeitig Nachteile für den Verarbeiter zum Tragen. Der Herstellungsprozess des Werkstoffes in zwei Schritten, wie er bei der Verwendung von PVAc-Leim notwendig ist, dauert wenigstens $32 \mathrm{~min}$ (reine Presszeit, Leimauftrag und Fügen der Furniere nicht einberechnet). Weiterhin müssen spezifische Topf- und offenen Zeiten eingehalten werden, ein aufwendigeres Applikationsverfahren (z. B. händisch mit Rakeln oder Walze; maschinell) ist notwendig. Zudem fallen Maschinen- und Werkzeugreinigungen an.

Die Verwendung des thermoplastischen Klebefilms liefert zwar die schlechteren Abhebefestigkeitswerte im Vergleich zur Anwendung von PVAc-Leim, dennoch sind die adhäsiven Eigenschaften sehr gut.

Als wesentlicher Nachteil dieses Klebstoffsystems ist die mangelnde Erfahrung in Bezug auf die adhäsiven Eigenschaften von Verklebungen unterschiedlicher Furniere mit "Collano 46.002“ zu nennen. Ein hoher Extraktstoffgehalt oder niedrige pH-Werte, wie sie besonders bei tropischen Hölzern anfallen (Fengel und Wegener 2003), könnten negative Einflüsse auf die Adhäsion haben.

Wie in Kapitel 4.4.1 intensiv untersucht konnte für das hier verwendete Eichenfurnier (im Vergleich zu Erlenfurnier) ein niedrigerer pH-Wert sowie ein deutlich erhöhter Extraktstoffgehalt festgestellt werden.

Die Verklebung der Erlenfurniere mittels des Klebefilms bietet jedoch eine einige Vorteile. Der Klebstoffilm muss nur auf die Größe der zu benetzenden Fläche zugeschnitten werden. Ein weiterer Maschinen- oder Geräteeinsatz ist nicht nötig und die Klebstoffmenge bleibt über die Fläche immer konstant. Es gibt keine Topf-oder offenen Zeiten und der Pressvorgang zur Herstellung des Verbundwerkstoffes dauert weniger als $3 \mathrm{~min}$ zzgl. einer Abkühlphase von ca. $5 \mathrm{~min}$. Werkzeuge müssen bei korrekter Anwendung diese Verfahrens ebenfalls nicht gereinigt werden.

Im Sinne eines möglichst ökonomischen, weil zeitsparenden Verfahrens, müsste die Verklebung des Absperrfurniers mit dem Deckfurnier mittels "Collano 46.002" bevorzugt werden. Die geringere Abhebefestigkeit im Vergleich zur Verwendung von PVAc ist insofern irrelevant, da sie immer noch deutlich höher ausfällt als die höchste Abhebefestigkeit zwischen Erlenfurnier und PVC-Folie (vergl. Kapitel 4.3.1und 4.3.5). 


\subsection{Vergleichende analytische Untersuchungen von Erlen- und Eichenfurnieren}

Um die Ursache für die signifikant schlechteren Abhebefestigkeiten von Eichenfurnier auf PVC-Folie gegenüber der analogen Versuchsreihe mit Erlenfurnier (vergl. 4.3.3) zu erklären, wurden in den folgenden Kapiteln analytische Untersuchungen zu den Extraktstoffen und der Oberflächentopographie beider Furnierarten durchgeführt.

\subsubsection{Vergleichende Untersuchung der Extraktstoffgehalte von Erlen- und Eichenfurnier}

In Kapitel 4.3.3 konnte festgestellt werden, dass sich die Abhebefestigkeiten und damit die adhäsiven Eigenschaften von Erlen- und Eichenfurnieren, die mittels PU-basiertem thermoplastischem Klebefilms auf PVC-Folie verklebt wurden, signifikant unterscheiden. Die Abhebefestigkeiten von Eichenfurnier auf PVC-Folie fielen hierbei geringer aus.

Als Ursache hierfür wurden unterschiedliche Extraktstoffmengen und pH- Werte der beiden Furnier-/Holzarten vermutet, da diese wie bereits in Kapitel 2.2.1 beschrieben einen erheblichen Einfluss auf die Qualität von Verklebungen haben können.

Um einen Vergleich zwischen den beiden Holzarten ziehen zu können, wurden die im Folgenden beschriebenen und in den Kapiteln 3.2.1.1 bis 3.2.1.3 erläuterten Heiß- und Kaltwasser-Extraktionen sowie die sukzessive Extraktion mittels Lösungsmitteln durchgeführt. Zunächst erfolgt eine Darstellung der Ergebnisse. Nachfolgend die Diskussion der jeweiligen Ergebnisse aus den einzelnen Extraktionsmethoden und im Anschluss eine gemeinsame Diskussion der durchgeführten Versuchsreihen.

4.4.1.1 Heißwasser- und Kaltwasserextraktion sowie sukzessive Extraktion von Erlen- und Eichenfurnier

Im folgenden Kapitel werden die Versuchsergebnisse der Heißwasser- und Kaltwasserextraktion sowie die der sukzessiven Extraktion von Erlen- und Eichenfurnier erläutert und im Anschluss diskutiert.

Tabelle 4-26 gibt einen Überblick zu den Versuchsergebnissen der Heiß- und Kaltwasserextraktion. 
Tabelle 4-26: Mittelwerte der Heiß- und Kaltwasserextrakte von Erlen- und Eichenfurnier unter Berücksichtigung der zugehörigen pH-Werte und alkalischen Pufferkapazitäten.

\begin{tabular}{|l|c|c|}
\hline & Erlenfurnier & Eichenfurnier \\
\hline Kaltwasserextraktion & 3,37 & 3,46 \\
\hline Kaltwasserextrakte [\%] & 4,68 & 3,85 \\
\hline pH-Wert & 1,14 & 3,57 \\
\hline Pufferkapazität [mmol NaOH] & & \\
\hline \multicolumn{2}{|l|}{} \\
\hline Heißwasserextraktion & 4,22 & 10,70 \\
\hline Heißwasserextrakte [\%] & 4,49 & 3,83 \\
\hline pH-Wert & 5,31 & 14,19 \\
\hline Pufferkapazität [mmol NaOH] &
\end{tabular}

Wie bereits durch die in Kapitel 2.2.1 erwähnten Veröffentlichungen (Roffael und Rauch 1974; Bodig und Jayne 1982; Grosser 2003) zu Extraktstoffen erwartet, zeigt sich, dass sich aus Eichenfurnieren sowohl durch Heißwasser- als auch Kaltwasserextraktion höhere Konzentrationen an Extraktstoffen als bei Erlenfurnieren lösen lassen.

Mittels Kaltwasserextraktion ließen sich bei Erlenfurnier Extraktstoffgehalte von 3,37 \% in den wässrigen Auszügen messen. Bei der Vergleichsuntersuchung von Eichenfurnier lösten sich 3,46 \% Extraktstoffe im Kaltwasser.

Die mittels pH-Elektrode in der abgefilterten Lösung (vergl. Kapitel 3.2.1.1) gemessenen $\mathrm{pH}-$ Werte differieren deutlich. Während der $\mathrm{pH}$-Wert der Erlenfurniere mit $\bar{x}=4,68$ gemessen wurde, beläuft er sich bei Eichenfurnieren auf $\bar{x}=3,85$.

Wie aus Tabelle 4-26 ersichtlich, steigen bei der Heißwasserextraktion sowohl die Extraktstoffgehalte (Erlenfurnier $=4,22 \%$; Eichenfurnier $=10,70 \%$ ) als auch die Pufferkapazitäten an, während die $\mathrm{pH}$-Werte weiter sinken (Erlenfurnier=4,49; Eichenfurnier $=3,83$ ) und die Acidität somit weiter steigt. Gerade der $\mathrm{pH}$-Wert und die Pufferkapazität können entscheidenden Einfluss auf die Verleimbarkeit von Hölzern oder Holzpartikeln haben (Popper 1974; Dunky 2002b).

Um eine größeren Umfang von Stoffklassen aus den Furnieren extrahieren zu können, wurde eine sukzessive Extraktion durchgeführt. Die hier verwendeten Lösungsmittel haben unterschiedliche Polaritäten und können somit verschiedene Stoffklassen lösen (vergl. Tabelle 4-28 und Faix 2012).

Tabelle 4-27 gibt einen Überblick zu den Mittelwerten der durch sukzessive Extraktion (vergl. Kapitel 3.2.1.3) und mittels verschiedener Lösungsmitteln bestimmten Extraktstoffgehalte von Eichen- und Erlenfurnieren. 
Tabelle 4-27: Nach Lösungsmitteln sortierte, mittlere Extraktstoffgehalte nach sukzessiver Extraktion von Erlen- und Eichenfurnier.

\begin{tabular}{|l|c|c|}
\hline \multirow{2}{*}{ Lösungsmittel } & \multicolumn{2}{|c|}{ Extraktstoffgehalt (\%) } \\
\cline { 2 - 3 } & Erlenfurnier & Eichenfurnier \\
\hline Petrolether & 0,14 & 0,17 \\
\hline Diethylether & 0,40 & 0,39 \\
\hline Aceton/Wasser $(9: 1)$ & 3,18 & 2,28 \\
\hline Ethanol/Wasser $(4: 1)$ & 0,99 & 1,72 \\
\hline Wasser $\left(60^{\circ} \mathrm{C}\right)$ & 0,82 & 1,23 \\
\hline
\end{tabular}

Wie aus Tabelle 4-27 ersichtlich, setzt sich der Trend der höheren Extraktstoffgehalte in Eichenfurnier weitgehend fort. Lediglich durch die Lösungsmittel Diethylether und dem Lösungsmittelgemisch aus Aceton und Wasser (im Verhältnis 9:1) wurden bei Erlenfurnier deutlich höhere Extraktstoffanteile gelöst. In der Extraktionsstufe mit Diethylether lag der mittlere Anteil der Extrakte aus Erlenfurnier bei 0,40\%, aus Eichenfurnier lösten sich $0,39 \%$ und somit auf einem ähnlichen Niveau.

Im Fall des Aceton/Wassergemischs (9:1) war der Unterschied an Extraktstoffgehalten wesentlich höher. Durch dieses Lösungsmittelgemisch konnten aus Erlenfurnier Extraktstoffanteile von $\bar{x}=3,18 \%$ gelöst werden, bei Eichenfurnier im Vergleich nur Anteile von $\bar{x}=2,28 \%$.

\section{Diskussion der Ergebnisse}

Ziel der Untersuchungen der Extraktstoffgehalte und des $\mathrm{pH}$-Wertes war es zu überprüfen, ob die in den Furnieren aus Erle und Eiche enthaltenen Holzextraktstoffe und der pH-Wert einen ungünstigen Einfluss auf die Klebergebnisse von Eichenfurnier, das mit PU-basiertem thermoplastischem Klebefilm auf PVC-Folie verklebt wurde, haben können (vergl. Kapitel 4.3.2).

Dieser Verdacht lag nahe, da sowohl Holzextraktstoffe als auch niedrige pH-Werte einen negativen Einfluss auf Verklebungen haben können (Popper 1974; Roffael und Rauch 1974; Roffael und Schäfer 2002; Fengel und Wegener 2003; Faix 2012; Dunky und Niemz 2002).

Wie sich gezeigt hat, unterscheiden sich auch die in dieser Arbeit untersuchten Abhebefestigkeiten von Erlen- und Eichenfurnier (verklebt auf PVC-Folie mittels PUbasierten thermoplastischen Klebefilms) signifikant voneinander. Die höchste gemessene mittlere Abhebefestigkeit lag bei Erlenfurnier bei $1,74 \mathrm{~N}^{*} \mathrm{~mm}^{-2}$ bei Eiche hingegen nur bei 1,64 $\mathrm{N}^{*} \mathrm{~mm}^{-2}$. Deutlich größer war die Differenz bei der optimalen Presszeit von $120 \mathrm{~s}$. Hier wurde bei Erlenfurnier eine mittlere Abhebefestigkeit von $1,71 \mathrm{~N}^{*} \mathrm{~mm}^{-2}$ gemessen, bei Eichenfurnier hingegen nur $1,48 \mathrm{~N}^{*} \mathrm{~mm}^{-2}$ (vergl. Kapitel 4.3.3). 
Wie durch die vorangegangenen Untersuchungen festgestellt werden konnte, unterscheidet sich der $\mathrm{pH}$-Wert deutlich, Eichenfurniere weisen einen niedrigeren $\mathrm{pH}$ Wert auf als die Erlenfurniere. Die im Rahmen dieser Arbeit gemessenen $\mathrm{pH}-$ Werte für Eichenfurniere entsprechen in etwa denen der Literatur (vergl. Fengel und Wegener 2003; Roffael und Rauch 1974). Ein exakter Vergleich mit Literaturwerten ist jedoch nur schwer möglich. Dies gilt auch für die unterschiedlichen Anteile an Extraktstoffen, da diese Werte mit dem Alter des Holzes, dem Standort, der Lage im Stamm u. ä. zum Teil stark variieren (Roffael und Rauch 1974; Roffael und Schäfer 2002; Faix 2012; Fengel und Wegener 2003).

Die für Erlenholz ermittelten Extraktstoffgehalte aus Kaltwasser- (3,37\%) und Heißwasserextraktion (4,22 \%) entsprechen in etwa der Angabe von Grosser 2003, der einen Extraktstoffgehalt von $5 \%$ nennt. Auch hier gilt, dass ein Vergleich der Werte schwierig ist, da die Extraktstoffgehalte durch o. g. Einflüsse stark schwanken können (Roffael und Rauch 1974; Roffael und Schäfer 2002; Faix 2012; Fengel und Wegener 2003).

In Bezug auf die Extraktstoffgehalte in verschiedenen Lösungsmitteln zeigte sich, dass eine klare Tendenz zu höheren gelösten Extrakstoffanteilen aus Eichenfurnier zu erkennen war.

Besondere Beachtung sollte dem hohen Unterschiede zwischen den Extraktstoffgehalten von Erlen- und Eichenfurnier in der Heißwasserextraktion geschenkt werden.

Weißmann 1984 beschreibt folgende Stoffgruppen als Extrakte im heißen Wasser: Hemicellulosen, monomere Zucker, Pektine und Hydroxistilbenglycoside sowie zu $50 \%$ Polyphenole.

Nach Fengel und Wegener 2003 werden hydrolisierbare und kondensierte Tannine der Gruppe der Polyphenole zugeordnet, die einen besonders starken Einfluss auf die Verklebung nehmen können.

Betrachtet man Tabelle 4-28 und setzt diese in Vergleich $\mathrm{zu}$ den ermittelten Ergebnissen die in Tabelle 4-27 dargestellt sind fällt auf, dass analog zu den zuvor erwähnten Ergebnissen der Heiß- und Kaltwasserextraktion die Extraktstoffgehalte von Eichenfurnier (im Vergleich zu Erlenfurnier), ermittelt durch die sukzessive Extraktion, in den Lösungsmitteln höher ausfallen, in denen die Stoffklasse der Tannine vertreten ist. Besonders deutlich zeigt sich dies bei den Lösungsmitteln Ethanol/Wasser (4:1) mit Werten von $\bar{x}=1,72 \%$ bei Eichenfurnier und $\bar{x}=0,99 \%$ bei Erle sowie bei Wasser $\left(60^{\circ} \mathrm{C}\right)$ mit Extraktstoffgehalten von $\bar{x}=1,23 \%$ bei Eichenfurnier und $\bar{x}=0,82 \%$ bei Erlenfurnier. 
Tabelle 4-28: Exktraktionsmittel der sukzessiven Exktraktion mit entsprechenden Stoffklassen im Extrakt (nach Faix 2012).

\begin{tabular}{|l|l|}
\hline Extraktionsmittel & Stoffklassen im Extrakt \\
\hline Petrolether & $\begin{array}{l}\text { freie Fettsäuren, Fette Sterine, } \\
\text { Sterinester, Kohlenwasserstoffe, Terpene }\end{array}$ \\
\hline Diethylether & partiell oxidierte Fette, Terpene \\
\hline Aceton/ Wasser & phenolische Verbindungen, Glycoside \\
\hline Enthanol/ Wasser & $\begin{array}{l}\text { monomere und oligomere Zucker, } \\
\text { Glycoside, z. T. niedermolekulare } \\
\text { Ligningvorstufen, Farbstoffe, Tannine }\end{array}$ \\
\hline Wasser $\left(60^{\circ} \mathrm{C}\right)$ & $\begin{array}{l}\text { Stärke und z. T. Hemicellulosen, } \\
\text { Farbstoffe, Tannine }\end{array}$ \\
\hline
\end{tabular}

Da die Extraktstoffgehalte bei Eichenfurnier besonders in den Lösemitteln höher ausfielen in denen die Stoffklassse der Tannine gelöst wird, gilt es dies genauer zu betrachten.

Tannine werden u. a. zwischen kondensierten und hydrolisierbaren Tanninen unterschieden (Fengel und Wegener 2003; Roffael und Schäfer 2002).

Im Zusammenhang mit dieser Arbeit scheinen besonders die hydrolisierbaren Tannine von Interesse, die in Eichenholz vorkommen, da diese einen maßgeblich negativen Einfluss auf die Verleimbarkeit und die Benetzbarkeit mit Bindemitteln haben können. Vornehmlich altes Eichenholz, wie es für die Furnierherstellung verwendet wird, besitzt einen höheren Gehalt an Extraktstoffen (Roffael und Rauch 1974; Fengel und Wegener 2003; Popper 1974). Verstärkt werden diese Fakten in Bezug auf die Fragestellung dieses Kapitels dadurch, dass hydrolisierbare Tannine durch Trocknung an die Holzoberfläche, die bei Furnieren besonders groß ist, wandern und somit einen negativen Einfluss auf die Aushärtung des Bindemittels haben können (Dunky 2002b, Christiansen 1991).

Die negativen Auswirkungen von Holzextraktstoffen auf Verklebungen sind besonders für formaldehydbasierte Klebstoffsysteme gut untersucht (Christiansen 1991; Roffael und Rauch 1974).

Im Rahmen dieser Arbeit konnte jedoch eine nachlassende Abhebefestigkeit bei Eichenfurnieren, die mit einem thermoplastischem Klebefilm (PU-basiert) auf PVCFolie verklebt wurden, festgestellt werden (vergl. Kapitel 4.3.2). Da für ein solches Klebstoffsystem (oder andere Klebstoffe auf PU-Basis) keine Veröffentlichungen zu ähnlichen Fragestellungen gefunden werden konnten, kann hier nur aufgrund des Kenntnisstandes bei anderen Klebstoffsystemen eine Hypothese abgeleitet werden.

Da bei der Untersuchung von Eichenfurnier im Vergleich zu Erlenfurnier ein deutlich niedrigerer $\mathrm{pH}$-Wert gemessen wurde und in den Lösungsmitteln, die besonders 
Tannine lösen, ein zum Teil deutlich höherer Extraktstoffgehalt festgestellt wurde, lässt sich vermuten, dass einer der beiden Faktoren oder beide gemeinsam für die schlechtere Abhebefestigkeit (vergl. Kapitel 4.3.2) von Eichenfurnier auf PVC-Folie verantwortlich sind. Um diese Hypothese zu bekräftigen sind allerdings intensivere und spezifischere Untersuchungen nötig.

\subsubsection{Vergleiche der Oberflächenrauigkeit von Erlen- und} Eichenfurnier

Schon bei der Betrachtung von Furnieren aus Erle und Eiche fällt auf, dass sich die Oberflächen deutlich voneinander unterscheiden. Eichenfurnier wirkt auch haptisch rau und strukturreich, während sich Furniere der Erle eher glatt und geschmeidig anfühlen. Dies liegt unter anderem daran, dass die Eiche zu den ringporigen Hölzern zählt, die Erle zu den zerstreutporigen (vergl. Kapitel 3.1.1) (Sachsse 1984).

Diese starke Struktur der Eiche, dies gilt auch für andere ringporige Laubhölzer, fällt besonders beim Tangentialschnitt auf. Hierfür sind insbesondere die großlumigen Frühholzgefäße verantwortlich (Sachsse 1984).

Bei zerstreutporigen Laubhölzern sind diese markanten Strukturen aufgrund der gleichmäßigen Verteilung von Früh- und Spätholzgefäßen und deren geringen und meist gleichbleibenden Durchmesser nicht vorhanden (Sachsse 1984).

Die in Abbildung 4-26 und Abbildung 4-27 dargestellten Topographien der Furnieroberfläche von Erle (Abbildung 4-26) und Eiche (Abbildung 4-27) zeigen deutlich die zuvor beschriebenen Unterschiede.
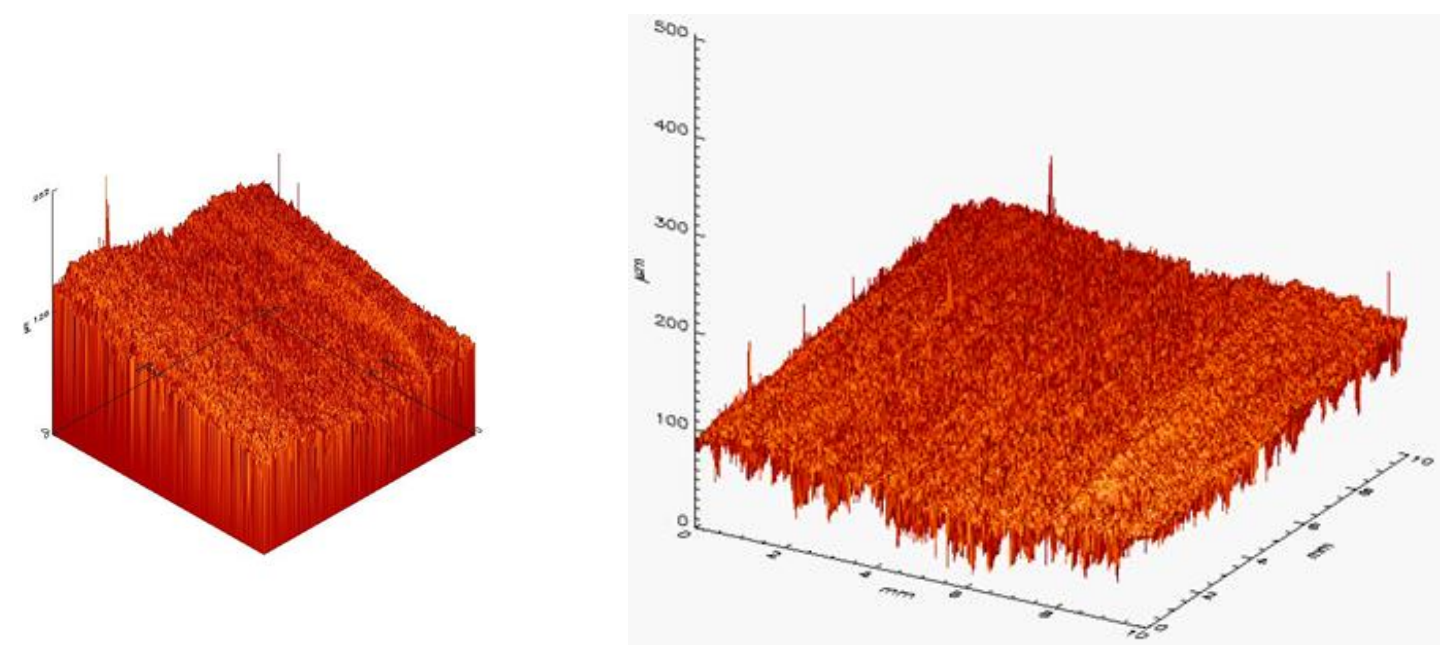

Abbildung 4-26: Darstellung der mittels „MicroProf" aufgenommenen Oberflächentopographie von Erlenfurnier. 
Während die Oberfläche des Erlenfurniers nahezu homogen wirkt und nur geringe „Krater" aufweist, sieht die Topographie von Eichenfurnier gänzlich different aus. Die Vertiefungen auf der Oberfläche von Erlenfurnier bleiben auf unter $100 \mu \mathrm{m}$ beschränkt, wohingegen bei Eichenfurnier vergleichsweise "tiefe Krater" von bis zu $250 \mu \mathrm{m}$ zu erkennen sind.

Daher stand die Annahme im Raum, dass die Beschaffenheit der Oberfläche des Eichenfurniers unter Umständen zu den im Vergleich zu Erlenfurnier schlechteren Ergebnissen bei den untersuchten Abhebefestigkeiten geführt haben könnten (vergl. Kapitel 4.3.2) .
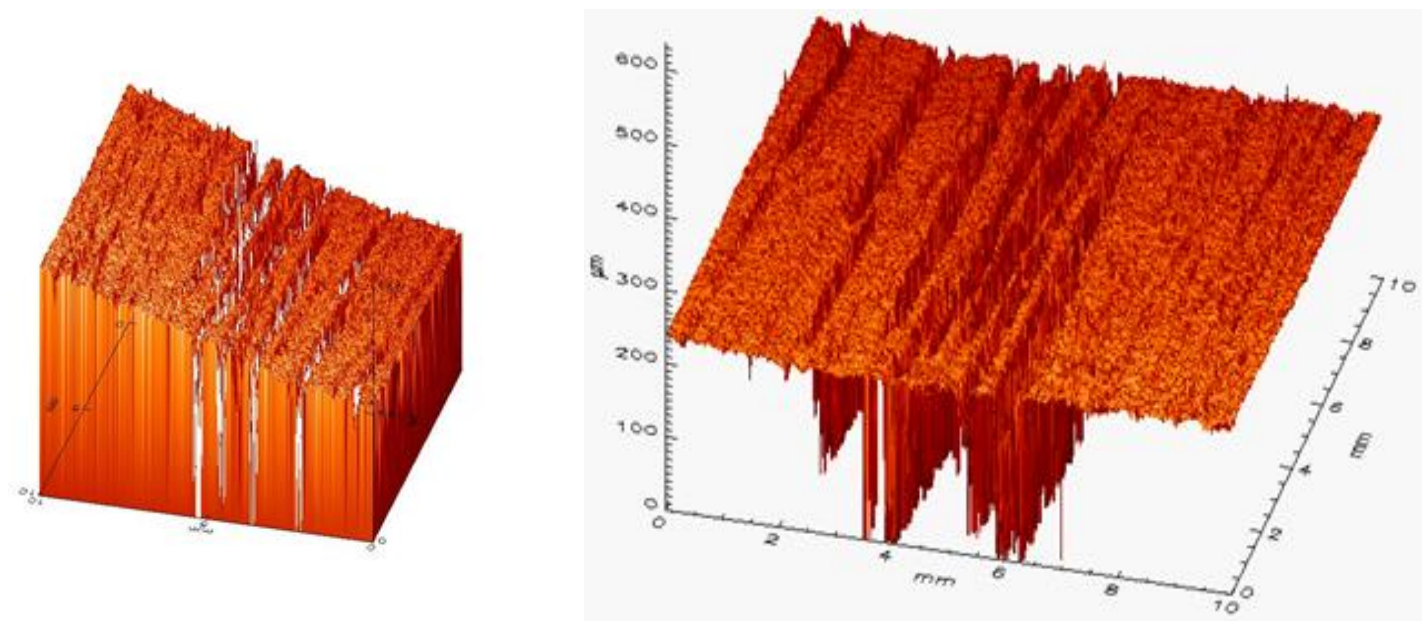

Abbildung 4-27: Darstellung der mittels „MicroProf" aufgenommenen Oberflächentopographie von Eichenfurnier.

Der Grund für die Annahme lag darin begründet, dass der äußerst dünne Klebefilm zu unzureichenden Klebstoffbenetzung aufgrund der rauen Oberflächenstruktur führen könnte, während PVAc-Leime wegen ihrer Beschaffenheit ein s. g. Fugenfüllvermögen besitzen (Dunky 2002a).

\section{Diskussion der Ergebnisse}

Während die topgraphischen Aufnahmen die Annahmen deutlicher Strukturunterschiede zwischen Eichen- und Erlenfurnier bekräftigen, kann in der Literatur kein eindeutiger Hinweis auf diese Annahme bestätigt werden.

Habenicht (2002) beschreibt drei verschiedene Arten von Oberflächen, die geometrische Oberfläche (gemessene Fügeteilbreit und Überlappungslänge), die wahre Oberfläche (Mikrooberfläche; ähnlich Abbildung 4-26 und Abbildung 4-27), die für klebtechnische Betrachtungen nur theoretischen Charakter hat sowie die wirksame Oberfläche (Anteil der wahren Oberfläche, der mit Klebstoff bedeckt wird). Die wirksame Oberfläche trägt somit maßgeblich zur Festigkeit der Klebung bei. 
Wie Habenicht (2002) weiter ausführt, haben bisherige systematische Versuche eine

Beziehung zwischen Klebefestigkeit und der Morphologie/Topographie von Oberflächen herzustellen zu dem Ergebnis geführt, dass die Intensität der technischen Rauheit und die Stärke der Haftfestigkeit in keinem eindeutigen Zusammenhang miteinander stehen.

Somit muss dieser Ansatz zwar nicht zwangsweise verworfen werden, ein Zurückstellen und ggf. intensiver Untersuchungen sind jedoch anzuraten.

\subsection{Werkstoffuntersuchungen des Gesamtverbundes/Halbzeugs}

In den vorangegangenen Kapiteln wurden sowohl die Materialien für den Verbundwerkstoff als auch die optimalen Herstellungsparameter festgelegt. Als optimale Materialkombination wurde Erlenfurnier auf PVC-Folie ermittelt.

Die ideale Presszeit für die höchsten Festigkeitswerte bei kürzester Verweildauer in der Presse beträgt 120 s. Grundsätzlich wurde in Kapitel 4.3 .5 festgestellt, dass der Gesamtverbund mit nur einem Pressvorgang (s. g. One-Shot-Verfahren) mittels des thermoplastischen Klebefilms „Collano 46.002“ hergestellt werden kann.

Die in den nächsten Kapiteln folgenden Untersuchungen wurden jedoch mit Probekörpern durchgeführt, deren Fertigung nach dem ursprünglich angedachten Herstellungsprozess geschah. Dies bedeutet, dass die Absperrfurniere mittels thermoplastischen Klebefilms auf die PVC-Folie aufgebracht wurden. Die Verklebung des Deckfurniers mit dem Absperrfurnier erfolgte durch Furnierleim auf PVAc-Basis. Der Grund hierfür lag an den Anforderungen, die der Projektpartner stellte.

Aufgrund der Versuchsergebnisse von Eichenfurnier auf PVC-Folie wurde der Wunsch geäußert, Deck- und Absperrfurnier grundsätzlich mit PVAc-Leim zu verkleben.

Der Grund hierfür liegt darin, dass der im Rahmen dieser Arbeit entwickelte Werkstoff für Produkte im Hochpreissegment Verwendung finden soll. In diesem Geschäftsbereich kommen sehr häufig Furniere aus tropischen Hölzern wie beispielsweise Makassar zum Einsatz. Im Vergleich zu Hölzern aus der gemäßigten Zone können Hölzer aus tropischen Baumarten einen hohen Anteil an Extraktstoffen beinhalten (Fengel und Wegener 2003). Holzextraktstoffe können nachweislich einen negativen Einfluss auf die Verleimbarkeit von Hölzern haben (vergl. Fengel und Wegener 2003, Roffael und Rauch 1974, Christiansen 1991). Da seitens des Projektpartners nicht für jede Furnier-/Holzart, die bei der Produktherstellung Verwendung findet, eine eigene Untersuchung auf die Qualität der Adhäsion 
durchgeführt werden kann, wird auf den (auch den angestellten Handwerkern) bekannten, im Umgang erprobten sowie bewährten PVAc-Leim zurückgegriffen.

\subsubsection{Biegefestigkeitsprüfung des Verbundwerkstoffes aus Erlenfurnier auf PVC-Folie}

Die Herstellungsparameter der Probeplatten zur Herstellung der Prüfkörper für die Bestimmung des Biege-Elastizitätsmoduls und der Biegefestigkeit nach DIN EN 310 können Tabelle 4-29 entnommen werden.

Tabelle 4-29: Herstellungsparameter der Faltfurnierplatten aus Erlenfurnieren und PVC-Folie zur Herstellung der Prüfkörper für die Biegefestigkeitsprüfung.

\begin{tabular}{|l|l|}
\hline Material & Messerfurniere Erle PVC-Folie \\
\hline Plattenformat & $750 \mathrm{~mm} \times 300 \mathrm{~mm}$ \\
\hline Furnierdicke & $\overline{\mathrm{x}}=0,54 \mathrm{~mm}$ \\
\hline Foliendicke & $\overline{\mathrm{x}}=0,91 \mathrm{~mm}$ \\
\hline Klebstoff MS-Absperrfurnier & Collano $46.002 / 100 \mathrm{~g}^{*} \mathrm{~m}^{-2}$ \\
\hline Klebstoff Furnier-Furnier & Kleiberit Furnierleim 322 \\
\hline Klebstoffmenge Furnierleim je Seite & $\overline{\mathrm{x}}=155,36 \mathrm{~g}^{*} \mathrm{~m}^{-2}$ \\
\hline Presstemperatur & $120{ }^{\circ} \mathrm{C}$ \\
\hline Pressdruck Heißpresse & $13,96 \mathrm{~N}^{*} \mathrm{~cm}^{-2}$ \\
\hline Pressdruck Furnierpresse (kalt) & $24,50 \mathrm{~N}^{*} \mathrm{~cm}^{-2}$ \\
\hline Schließzeit Presse & $\overline{\mathrm{x}}=8,83 \mathrm{~s}$ \\
\hline
\end{tabular}

Der Unterschied zu den vorherigen Herstellungsparametern der Probeplatten liegt in der Verwendung des PVAc-Leims „Kleiberit 322“ zur Verklebung des Deckfurniers auf dem Absperrfurnier. Die mittlere verwendete Leimmenge je Seite liegt mit $\bar{x}=155,36 \mathrm{~g}^{*} \mathrm{~m}^{-2}$ im Bereich der Empfehlungen des Klebstoffherstellers, der diesen mit einer Auftragsmenge von $120 \mathrm{~g}^{*} \mathrm{~m}^{-2}-180 \mathrm{~g}^{*} \mathrm{~m}^{-2}$ angibt (KLEBCHEMIE M. G. Becker GmbH \& Co. KG 1999).

Auch der höhere Pressdruck von 24,50 $\mathrm{N}^{*} \mathrm{~cm}^{-2}$, der beim Verpressen mit Furnierleim angewendet wurde, entspricht den Empfehlungen des Herstellers. Dieser rät zu einem Pressdruck von $0,20 \mathrm{~N}^{*} \mathrm{~mm}^{-2}$ bis 0,50 N*mm-2 (vergl. Kapitel 4.1) (KLEBCHEMIE M. G. Becker GmbH \& Co. KG 1999).

Tabelle 4-30 stellt die Ergebnisse der Biegefestigkeitsprüfung des Gesamtverbundes aus Erlenfurnieren auf PVC-Folie dar. Die Ergebnisse zur Biegefestigkeit und des Biege-E-Modul sind, bezogen auf den Faserverlauf des Deckfurniers zum Prüfstempel, entsprechend DIN EN 310 nach „senkrecht“ und „parallel“ unterteilt. 
Tabelle 4-30: Vergleichende Ergebnisse der Biegefestigkeitsprüfungen des Gesamtverbundes sowie Sortierung der Ergebnisse nach Ausrichtung der Holzfaser des Deckfurniers.

\begin{tabular}{|l|c|c|c|c|}
\hline Parameter & $\begin{array}{c}\text { Biegefestig- } \\
\text { keit } \\
\text { "senkrecht" } \\
\mathbf{n = 2 4} \\
{\left[\mathbf{N}^{*} \mathbf{m m}^{-2}\right]}\end{array}$ & $\begin{array}{c}\text { Biege-E- } \\
\text { Modul } \\
\text { "senkrecht" } \\
\mathbf{n = 2 4} \\
{\left[\mathbf{k N}^{*} \mathbf{m m}^{-2}\right]}\end{array}$ & $\begin{array}{c}\text { Biegefestig- } \\
\text { keit } \\
\text { "parallel" } \\
\mathbf{n = 2 2} \\
{\left[\mathbf{N}^{*} \mathbf{m m}^{-2}\right]}\end{array}$ & $\begin{array}{c}\text { Biege-E- } \\
\text { Modul } \\
\text { "parallel" } \\
\mathbf{n = 2 2} \\
{\left[\mathbf{k N}^{*} \mathbf{m m}^{-2}\right]}\end{array}$ \\
\hline Presszeit s & 120 & 120 & 120 & 120 \\
\hline Median Z & 47,46 & 5,88 & 27,88 & 2,00 \\
\hline $\begin{array}{l}\text { Arithmetisches } \\
\text { Mittel } \boldsymbol{\mu}\end{array}$ & 47,42 & 5,87 & 28,44 & 2,01 \\
\hline $\begin{array}{l}\text { Standardab- } \\
\text { weichung } \mathbf{S}\end{array}$ & 2,93 & 0,40 & 2,55 & 0,12 \\
\hline Minimum & 42,76 & 5,19 & 25,37 & 1,76 \\
\hline Maximum & 54,70 & 6,60 & 34,19 & 2,25 \\
\hline
\end{tabular}

Die Überprüfung der Biegefestigkeit der Versuchsreihe „senkrecht“ ergab einen Wert von $\overline{\mathrm{x}}=47,42 \mathrm{~N}^{*} \mathrm{~mm}^{-2}$ bei einer errechneten Standardabweichung von $2,93 \mathrm{~N}^{*} \mathrm{~mm}^{-2}$. Entsprechend nahe liegen auch die Extremwerte mit einem Minimum von $42,76 \mathrm{~N}^{*} \mathrm{~mm}^{-2}$ und einem Maximum von $54,20 \mathrm{~N}^{*} \mathrm{~mm}^{-2}$ am arithmetischen Mittel von $47,42 \mathrm{~N}^{*} \mathrm{~mm}^{-2}$.

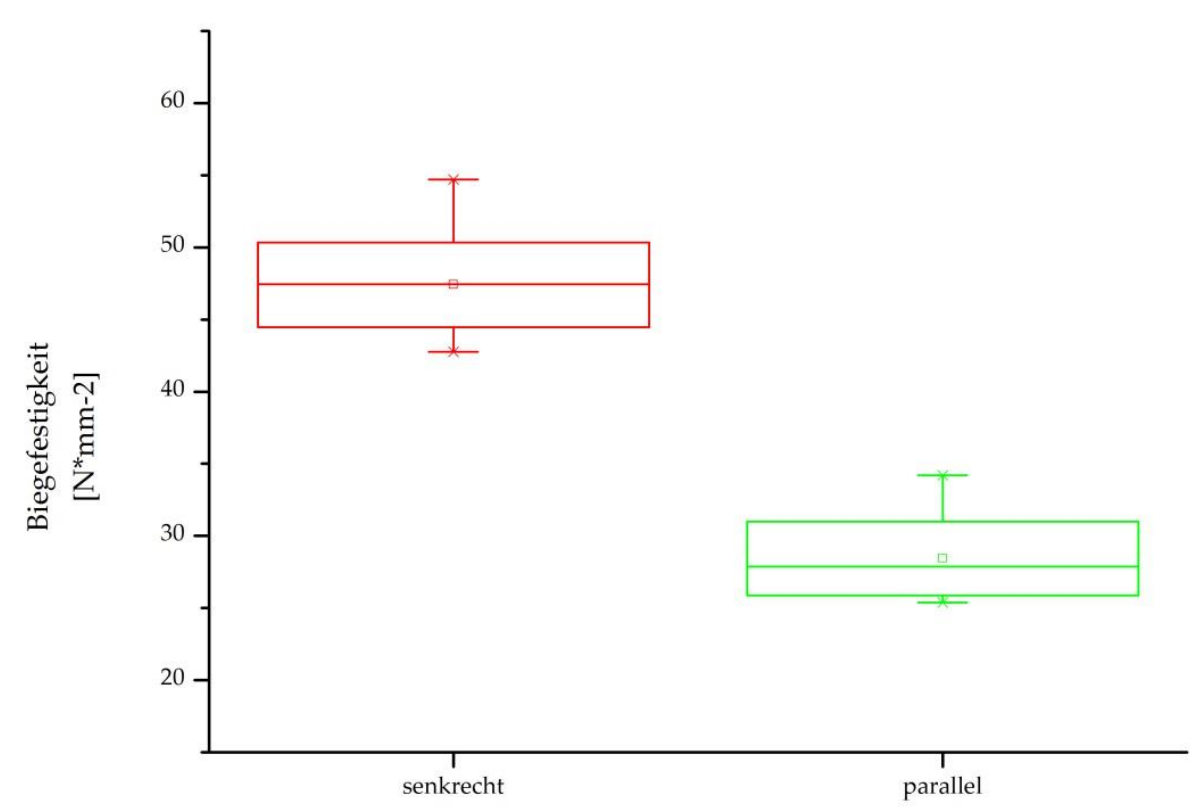

Abbildung 4-28: Boxplots zur Biegefestigkeit des Gesamtverbundes aus Erlenfurnieren auf PVC-Folie, sortiert nach der Faserausrichtung des Deckfurniers zum Prüfstempel. 
In Bezug auf die Streuparameter lieferte auch die Biegefestigkeitsprüfung der Versuchsreihe „parallel“ ein ähnliches Bild. Bei einem Mittelwert der Biegefestigkeit von $28,44 \mathrm{~N}^{*} \mathrm{~mm}^{-2}$ und einer Standardabweichung von $2,55 \mathrm{~N}^{*} \mathrm{~mm}^{-2}$ streuen die Extrema mit einem Minimum von 25,37 $\mathrm{N}^{*} \mathrm{~mm}^{-2}$ und einem Maximum von $34,19 \mathrm{~N}^{*} \mathrm{~mm}^{-2}$ zwar etwas stärker als bei "senkrecht", aber dennoch „nahe“ um das arithmetische Mittel, was auch Abbildung 4-28 anschaulich darstellt.

Die Überprüfung des Biege-E-Modul liefert ein analoges Bild $\mathrm{zu}$ den Versuchsergebnissen der Biegefestigkeitsprüfung.

Der Biege-E-Modul liegt bei der Versuchsreihe "senkrecht“ mit $\bar{x}=5,87 \mathrm{kN}^{*} \mathrm{~mm}^{-2}$ und einer Standardabweichung von $0,40 \mathrm{kN}^{*} \mathrm{~mm}^{-2}$ deutlich über dem Biege-E-Modul der Versuchsreihe "parallel“ mit einem Ergebnis von $\bar{x}=2,01 \mathrm{kN}^{*} \mathrm{~mm}^{-2}$ und einer errechneten Standardabweichung von $\overline{\mathrm{x}}=0,12 \mathrm{kN}^{*} \mathrm{~mm}^{-2}$.

Wie aus Abbildung 4-29 ersichtlich, streuen auch hier die Extremwerte nur vergleichsweise eng um die arithmetischen Mittel. In der Versuchsreihe „senkrecht" wurde ein Minimum von $5,19 \mathrm{kN}^{*} \mathrm{~mm}^{-2}$ und ein Maximum von $6,60 \mathrm{kN}^{*} \mathrm{~mm}^{-2}$ gemessen. Die Extrema der Versuchsreihe "parallel“ betrugen im Minimum $1,76 \mathrm{kN}^{*} \mathrm{~mm}^{-2}$ und im Maximum 2,25 kN* $\mathrm{mm}^{-2}$.

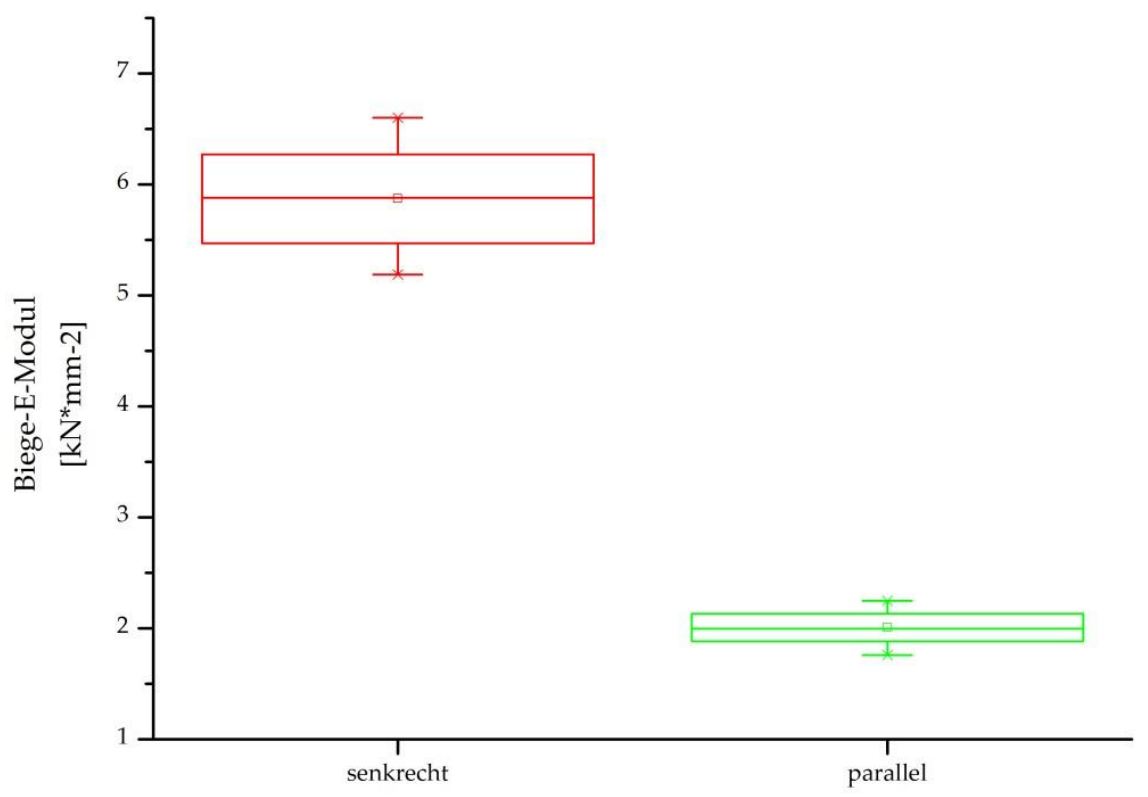

Abbildung 4-29: Boxplots zum Biege-E-Modul des Gesamtverbundes aus Erlenfurnieren auf PVCFolie, sortiert nach der Faserausrichtung des Deckfurniers zum Prüfstempel.

Wie auch in den vorangegangenen Kapiteln werden folgende zwei Hypothesen aufgestellt: 
$\mathrm{H}_{0}$ : Die jeweiligen arithmetischen Mittelwerte der Biegefestigkeit und des Biege-EModul, bezogen auf die Faserausrichtung des Deckfurniers zum Prüfstempel, unterscheiden sich nicht.

$\mathrm{H}_{1}$ : Die jeweiligen arithmetischen Mittelwerte der Biegefestigkeit und des Biege-EModul, bezogen auf die Faserausrichtung des Deckfurniers zum Prüfstempel, unterscheiden sich signifikant.

Obwohl Abbildung 4-28 und Abbildung 4-29 deutlich von einer Annahme von $\mathrm{H}_{1}$ ausgehen lassen, wurde dies noch einmal statistisch überprüft.

Der Mittelwertvergleich der Biegefestigkeiten der Versuchsreihen „senkrecht" und „parallel“ mittels Kruskal-Wallis-Test ergab bei einem Signifikanzniveau von 0,05, dass sich die Mittelwerte der beiden Grundgesamtheiten signifikant unterscheiden.

Die Mittelwerte der Biege-E-Moduln aus den Versuchsreihen "senkrecht" und „parallel“ wurden mittels ANOVA verglichen. Bei einem Signifikanzniveau von 0,05 unterscheiden sich diese beiden Grundgesamtheiten ebenfalls signifikant.

Somit muss sowohl für die Untersuchungen der Biegefestigkeit als auch die des BiegeE-Modul $\mathrm{H}_{0}$ verworfen und $\mathrm{H}_{1}$ angenommen werden.

\section{Diskussion der Versuchsergebnisse}

Die Bestimmung des Biege-E-Moduls und der Biegefestigkeit zeigten, dass der nur etwas über $3 \mathrm{~mm}$ dicke Werkstoff hervorragende Festigkeitseigenschaften hinsichtlich der beiden genannten Größen aufweist. Da die Versuchsergebnisse nur sehr wenig streuen, ist zudem von validen Werten auszugehen.

Um die Ergebnisse besser einordnen zu können, sollen diese mit den Biegefestigkeiten und E-Moduln anderer holzbasierter Werkstoffe verglichen werden.

Tabelle 4-31: Vergleichende Werte von Faltfurnierplatten zu anderen holzbasierten Werkstoffen in Bezug auf die Biegefestigkeit und den Biege-E-Modul (Werte für die Werkstoffe entnommen aus (Niemz 2002a; Niemz und Sonderegger 2017)).

\begin{tabular}{|c|c|c|c|c|c|c|}
\hline $\begin{array}{l}\text { Werkstoff- } \\
\text { eigenschaft }\end{array}$ & Einheit & $\begin{array}{c}\text { Falt- } \\
\text { furnier }\end{array}$ & $\begin{array}{c}\text { Span- } \\
\text { platte }\end{array}$ & MDF & $\begin{array}{c}\text { OSB } \\
\text { (Europa) }\end{array}$ & $\begin{array}{c}\text { Baufurniersperr- } \\
\text { holz } \\
\text { (Buche; > 5 Lagen) }\end{array}$ \\
\hline Werkstoffdicke & $\mathrm{mm}$ & $\overline{\mathrm{x}}=3,07$ & & & & \\
\hline Biegefestigkeit & $\mathrm{N}^{*} \mathrm{~mm}^{-2}$ & & $20-22$ & $33-38$ & & \\
\hline senkrecht & & 47,42 & & & $20-25$ & $5-17$ \\
\hline parallel & & 28,44 & & & 36,00 & $18-29$ \\
\hline Biege-E-Modul & $\mathrm{kN}^{*} \mathrm{~mm}^{-2}$ & & $2,6-3,2$ & $4,0-4,5$ & & \\
\hline senkrecht & & 5,87 & & & 1,85 & $0,65-4,00$ \\
\hline parallel & & 2,01 & & & 7,00 & $5,9-9,6$ \\
\hline
\end{tabular}

Wie aus Tabelle 4-31 deutlich hervorgeht, können die Werte der Biegefestigkeit des im Rahmen dieser Arbeit entwickelten Werkstoffes, der lediglich eine Materialdicke von 
etwas über $3 \mathrm{~mm}$ aufweist, mit denen anderer Holzwerkstoffe, die im Baubereich Verwendung finden, durchaus mithalten oder übertreffen sie je nach Prüfrichtung deutlich. Die Biegefestigkeitswerte der bisher zum Vergleich herangezogenen Spanplatte liegen mit angegebenen $20,00 \mathrm{~N}^{*} \mathrm{~mm}^{-2}$ bis $22,00 \mathrm{~N}^{*} \mathrm{~mm}^{-2}$ noch ca. $6,00 \mathrm{~N}^{*} \mathrm{~mm}^{-2}$ unterhalb der schwächeren Prüfrichtung („parallel“) von der Faltfurnierplatte. Auch die Biegefestigkeit des ebenfalls aus Furnieren hergestellten Baufurniersperrholz aus Buche liegt (von den höchsten Werten ausgehend) in der Prüfrichtung "senkrecht" ca. 30,00 N*mm-2 unterhalb der Festigkeitswerte von Faltfurnierplatte. In der Prüfrichtung „parallel“ sind die Werte nahezu identisch.

Hier zeigt sich, dass die Verwendung dünner Messerfurniere mit durchschnittlich 0,54 mm Stärke die Werkstoffeigenschaften hinsichtlich der Festigkeit positiv beeinflussen. Niemz und Sonderegger 2017 beschreiben für Lagenholz, dass mit zunehmender Furnierdicke die Festigkeit nachlässt. Im Umkehrschluss also dünnere Furniere die Festigkeit steigern. Sie bewerten diesen Einfluss als „deutlich“.

Somit ist die Biegefestigkeit für die seitens des Projektpartners zunächst angedachten Endprodukte (vergl. Kapitel 1.1) völlig ausreichend und eröffnet daher die Möglichkeit über weitere Produkte/Produktgruppen aus Faltfurnier nachzudenken. Der limitierende Faktor bezüglich senkrecht wirkender Kräfte (z. B. Flaschen im Flaschenträger, wie in Abbildung 4-2 dargestellt) ist demgemäß nicht die Biegefestigkeit des Halbzeugs, sondern die signifikant reduzierte Zugfestigkeit der PVC-Folie im angewinkelten Zustand, wie in Kapitel 4.2.3 beschrieben.

Der Biege-E-Modul des Werkstoffes Faltfurnier liegt mit 5,87 kN*mm-2 (,,senkrecht“) und 2,01 kN*mm-2 (,parallel“) zwischen den Werten von Spanplatten mit 2,6 kN*mm-2 bis $3,2 \mathrm{kN}^{*} \mathrm{~mm}^{-2}$ und OSB mit 1,85 $\mathrm{kN}^{*} \mathrm{~mm}^{-2}$ (senkrecht) und 7,00 $\mathrm{kN}^{*} \mathrm{~mm}^{-2}$ (parallel). 


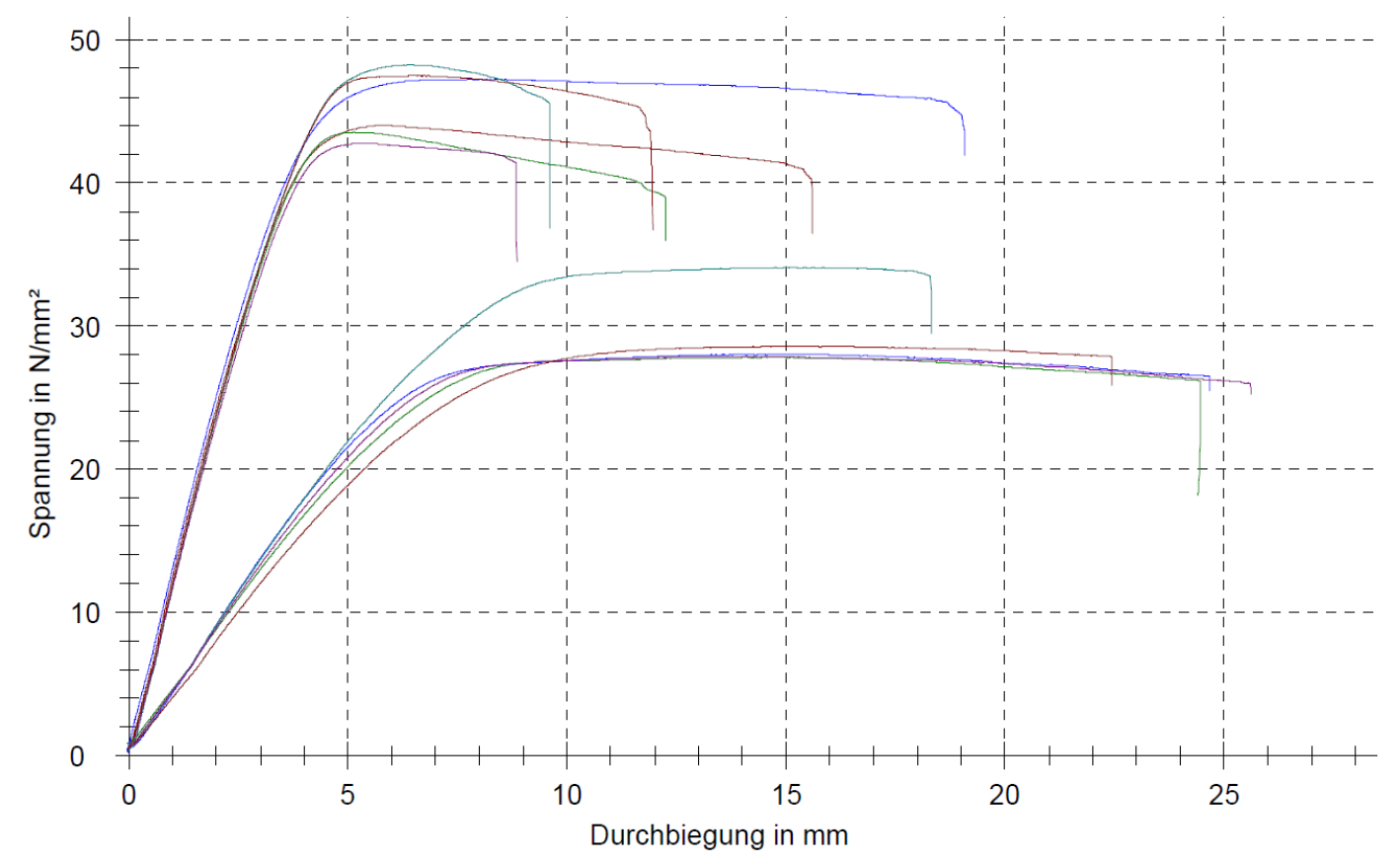

Abbildung 4-30: Seriengrafik einer Biegefestigkeitsversuchsreihe von Faltfurnier. Die unteren Kurven gehören zur Versuchsreihe "parallel" die oberen zu "senkrecht".

Abbildung 4-30 zeigt deutlich die Unterschiede zwischen den Biege-E-Moduln der Versuchsreihen „senkrecht" und „parallel“ von Faltfurnier. Die Durchbiegung bzw. Dehnung der Versuchsreihe „parallel“ bis zu 16 mm länger im Vergleich zur Serie „senkrecht“. Hier kommt die elastische Wirkung der PVC-Folie deutlich zum Tragen.

\subsubsection{Dauerhaftigkeitsuntersuchungen der Faltnut}

Das wesentliche Merkmal des im Rahmen dieser Arbeit entwickelten Werkstoffes ist seine Eigenschaft der wiederkehrenden Faltbarkeit. Diese Funktion wird zum einen durch die flexible Mittellage in Form der PVC-Folie und zum anderen durch das beidseitige, symmetrische Einfräsen der Furnierschichten auf eben diese Mittellage realisiert.

Dementsprechend übernimmt die Mittelschicht die Funktion eines Scharniers. Durch das Einfräsen der Furnierschichten fehlt an diesen Stellen eine aussteifende und stabilisierende Furnierschicht. Dies führt dazu, dass eben diese Nut, im Folgenden aufgrund ihrer Funktion „Faltnut" genannt, auch der Schwachpunkt des Werkstoffes ist. Je nach Produkt, z. B. Aktenmappe oder Flaschenträger (vergleiche dazugehörige Abbildungen im Anhang), sind Faltvorgänge einmalig beim Zusammenbau oder häufig möglich.

Vor allem bei Produkten, die nur einmalig zum Zusammenbau gefaltet werden, ist die Zugfestigkeit der ausschlaggebende und limitierende Faktor (vergl. Kapitel 4.2). 
Bei Produkten wie Visitenkartenetuis, Aktenmappen o. ä. aus dem Werkstoff Faltfurnier finden je nach Intensität des Gebrauchs häufig Faltvorgänge in Form von Öffnen und Schließen statt. Hier kommt weniger die statische Belastung als vielmehr die dynamische Belastung zum Tragen.

Da ein Produkt möglichst langlebig sein soll, muss es einer gewissen Anzahl von Faltvorgängen standhalten. Diese Anzahl wurde in Kapitel 1.2 mit minimal $1.000 \mathrm{zu}$ erreichenden Faltvorgängen definiert.

Um die maximal möglichen Faltvorgänge zu untersuchen wurde ein Versuchsaufbau wie in Kapitel 3.2.2.8 beschrieben getätigt.

Ähnliche Versuchsanordnungen sind auch aus der Textil- und Papierindustrie bekannt. Der Widerstand gegen Biegen, Falzen und Knittern wird dort Falzfestigkeit genannt und gilt bis zu einem bestimmten Grad als Maß für die Brüchigkeit eines Materials (Heermann und Herzog 1931).

Tabelle 2-1 gibt einen Überblick über diese Dauerhaftigkeitsüberprüfungen der Faltnut.

Tabelle 4-32: Ergebnisse der Dauerhaftigkeitsprüfung, in Bezug auf maximal mögliche Faltintervalle, des Gesamtverbundes aus Erle mit PVC-Folie.

\begin{tabular}{|l|c|}
\hline Parameter & $\begin{array}{c}\text { Faltvorgänge } \\
\text { [Wiederholungen] }\end{array}$ \\
\hline Stichprobenumfang $\mathrm{n}$ & 15 \\
\hline Faltwinkel & $90^{\circ}$ \\
\hline Median $\mathbf{Z}$ & 4.860 \\
\hline Arithmetisches Mittel $\boldsymbol{\mu}$ & 4.441 \\
\hline Standardabweichung S & 1.057 \\
\hline Minimum & 2.491 \\
\hline Maximum & 5.700 \\
\hline
\end{tabular}

Im Mittel wurden 4.441 Faltvorgänge bis zum Versuchsabbruch oder einem Materialversagen erreicht. Auffällig ist jedoch die hohe Standardabweichung von 1.057 Faltvorgängen sowie die Differenz zwischen dem Minimum mit 2.491 und dem Maximum von 5.700 Faltvorgängen.

Um mögliche Unterschiede in der Höhe der erreichten Faltintervalle interpretieren zu können, wurde jeder Probekörper auf mögliche Beschädigungen der Mittelschicht durch den Fräskopf untersucht und eine Messung der tatsächlichen Materialstärke der Mittelschicht durchgeführt.

\section{Diskussion der Versuchsergebnisse}

Zunächst lässt sich feststellen, dass die Minimalanforderung in Form von 1.000 möglichen Faltintervallen beim Faltfurnier aus Erlenfurnier und PVC-Folie problemlos erreicht wurde. 
Als unbefriedigend stellt sich jedoch die Situation der starken Streuung der Werte dar. Bei lediglich 15 untersuchten Probekörpern herrscht zwischen dem Minimum und Maximum eine Differenz von 3.209 Faltintervallen.

Wie der Vergleich der einzelnen Dickenmessungen der Mittelschicht mit den zugehörigen Probekörpern zeigte, lies die Anzahl der erreichten Faltintervalle in direktem Zusammenhang mit dem Materialverlust durch ein zu tiefes Einfräsen nach. Somit konnte belegt werden, dass ein Verringern der Materialstärke der Mittelschicht durch Fräsbeschädigungen zu einem Nachlassen der Dauerhaftigkeit führt (vergl. auch (Werndl 2014). Dies heißt jedoch nicht, dass eine dünnere Mittelschicht grundsätzlich weniger dauerhaft wäre.

Es empfiehlt sich demnach, abgeleitet aus den Versuchsergebnissen, ein besonderes Augenmerk auf das Aufspannen des Halbzeugs auf den Frästisch zu legen. Somit ist streng darauf $\mathrm{zu}$ achten, dass das Halbzeug vor dem Fräsvorgang absolut flach positioniert wird.

Zusätzlich könnte es empfehlenswert sein, die zur Produktherstellung benötigten Fräsmaschinen (Bearbeitungszentren) mit s. g. Tastaggregaten auszustatten, die Unterschiede in den Plattendicken oder Ungenauigkeiten beim Aufspannen auf den Frästisch ausgleichen können und das Fräswerkzeug somit immer auf der exakt vorgegeben Tiefe arbeiten lassen (vergl. z. B. Werkzeuge der Firma Benz, Haslach i. K.).

\subsubsection{Lagerung des Verbundwerkstoffes in starkem Wechselklima}

Um einen Eindruck der Formstabilität des Werkstoffes bei sich wechselnden klimatischen Bedingungen zu erhalten, wurde der Verbund aus Erlenfurnieren auf PVC-Folie für mehr als drei Monate bei sich stark ändernden Bedingungen in einer Klimakammer gelagert.

Da Holz ein hygroskopisches Material ist, kommt es zu Anpassungen der Holzfeuchtigkeit an die Luftfeuchte. Da es mit der Aufnahme von Feuchtigkeit, aber auch mit der Abgabe von Wasser an die Umgebungsluft zu Formveränderungen des Holzes kommt (Quellen und Schwinden) (Annies 2010), waren die Schwankungen der Plattengewichte von Interesse.

Die Formstabilität wurde im Anschluss an die Klimalagerung lediglich optisch beurteilt. Hierzu erfolgte eine Begutachtung der Faltfurnierplatten auf etwaige Risse, Verwerfungen in den Furnieren, Schüsselungen etc.

Tabelle 4-33 gibt einen Überblick zu den Parametern der Klimalagerung. 
Tabelle 4-33: Übersicht zur Lagerzeit, der Temperatur, und der Luftfeuchtigkeit der Klimakammer sowie den sich ändernden Gewichten der Probeplatten.

\begin{tabular}{|l|c|c|c|}
\hline Zeitraum & $\begin{array}{c}\text { mittleres } \\
\text { Plattengewicht } \\
(\mathbf{g})\end{array}$ & $\begin{array}{c}\text { Temperatur } \\
\left({ }^{\circ} \mathbf{C}\right)\end{array}$ & $\begin{array}{c}\text { Luftfeuchtigkeit } \\
\mathbf{( \% )}\end{array}$ \\
\hline $01.10 .2013-15.10 .2013$ & 542,50 & 20 & 65 \\
\hline $15.10 .2013-21.11 .2013$ & 561,63 & 5 & 90 \\
\hline $21.11 .2013-12.12 .2013$ & 559,80 & 50 & 90 \\
\hline $12.12 .2013-07.01 .2014$ & 519,90 & 50 & 10 \\
\hline $07.01 .2014-10.01 .2014$ & 541,07 & 20 & 65 \\
\hline
\end{tabular}

Die höchste Gewichtsschwankung fand zwischen einer Raumtemperatur von $50{ }^{\circ} \mathrm{C}$ bei $10 \%$ Luftfeuchtigkeit und $5{ }^{\circ} \mathrm{C}$ und $90 \%$ Luftfeuchtigkeit statt. Die Differenz des Plattengewichts betrug hier 41,73 g. Die höchste Differenz zwischen dem Gewicht bei Normalklima $\left(20^{\circ} \mathrm{C}\right.$ bei $65 \%$ Luftfeuchtigkeit) belief sich auf $19,13 \mathrm{~g}$ im Vergleich zu $5{ }^{\circ} \mathrm{C}$ bei $90 \%$ Luftfeuchtigkeit. Die Gewichtsveränderung lag somit bei 3,53 Prozent zum Ausgangsgewicht.

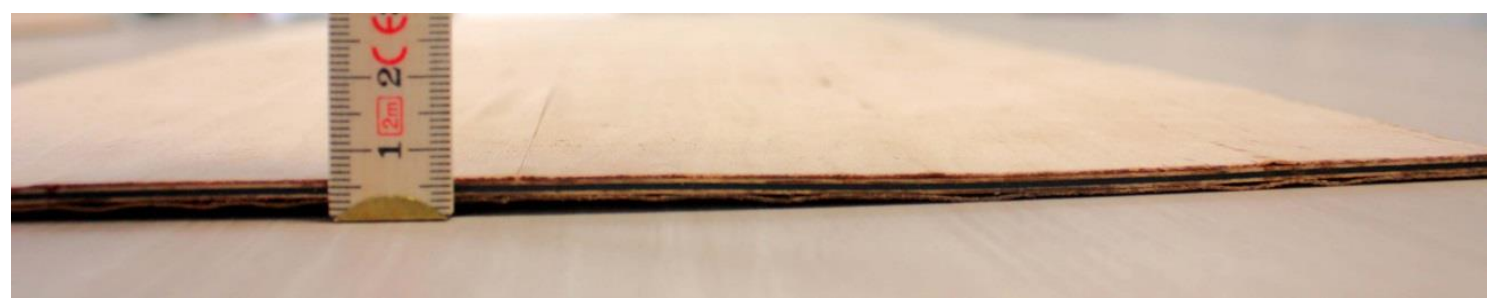

Abbildung 4-31: Foto der Faltfurnierplatte mit den auffälligsten Veränderungen nach der Lagerung im Wechselklima.

Wie Abbildung 4-31 zeigt, wurden die Ränder der Faltfurnierplatte um lediglich $2 \mathrm{~mm}$ durch die Lagerung im starken Wechselklima geworfen. Die in dieser Abbildung zu erkennenden leichten Wellen des Deckfurniers rühren daher, dass der verwendete Leim Kleiberit 322 nur für eine gelegentliche und kurzzeitige Exposition mit Wasser geeignet ist. Da die Klebefugen von nicht wasserfesten PVAc-Leimen bei zu hoher Feuchtigkeit quellen, verlieren sie an Festigkeit (Dunky 2002a), was zu den oben erwähnten Wellen im Deckfurnier führte.

Grundsätzlich zeigten die untersuchten Faltfurnierplatten eine gute Formstabilität, die nötigenfalls durch den Einsatz von wasserresistenten PVAc-Leimen zur Verklebung des Absperrfurniers mit dem Deckfurnier noch weiter verbessert werden könnte.

Diese gute Formstabilität der Faltfurnierplatten kann durchaus auf die Verwendung von Erlenfurnieren zurückzuführen sein. Das Holz der Erle gilt als mäßig schwindend und sein Stehvermögen nach Austrocknung wird als ausgezeichnet beschrieben. Zudem gehört Erle zu den Holzarten, die weder zum Reißen noch zum Schwinden neigen (Grosser 2003). 


\section{Zusammenfassung}

Die steigende Nachfrage nach sowohl langlebigen, nachhaltig sowie regional und fair produzierten Produkten steigt seit Anfang der 2000er Jahre stetig an. Insbesondere Produkte aus Holz erfreuen sich immer größerer Beliebtheit.

Vor diesem Hintergrund entstand gemeinsam mit der Firma Josef Wochner GmbH \& Co. $K G$, Rosenfeld Heiligenzimmern die Idee, einen Werkstoff auf Basis von Echtholzfurnieren zu entwickeln, der im Verpackungsumfeld eingesetzt werden kann.

Hierzu wurde das Konzept entworfen Furniere mit einer flexiblen Mittelschicht zu verkleben, um nach beidseitigem symmetrischen Einfräsen der Furnierschicht eine Art Scharnierfunktion durch die Mittelschicht zu erhalten. Auf diese Weise sollte es möglich sein, aus einem zunächst flachen Werkstoff 3-dimensionale Objekte ähnlich eines Umzugskartons, falten zu können. Somit wäre das Endprodukt zunächst flach lager- und auslieferbar, um anschließend vor Ort zusammengefaltet zu werden.

Das Hauptziel dieser Arbeit war die Entwicklung eines solchen Verbundwerkstoffes auf Furnierbasis und des dazugehörigen Herstellungsprozesses.

Um eine möglichst hohe Dimensionsstabilität zu gewährleisten, wurde ein 5schichtiger Aufbau aus jeweils zwei kreuzverleimten Furnierblättern auf einer flexiblen Mittelschicht angestrebt.

Dieser Verbundwerkstoff, Faltfurnier genannt, seine Teilverbünde sowie seine Bestandteile wurden hinsichtlich ihrer mechanisch-technologischen Eigenschaften untersucht und bewertet. Bei den zunächst zur Verwendung angedachten Furnieren aus Erle (Alnus glutinosa L) und Eiche (Quercus petraea (MATT.) LIEBL. bzw. Quercus robur L.) wurden zusätzlich die chemisch-physikalischen Merkmale betrachtet.

Nach diversen Vorversuchen wurde eine Materialkombination aus den o. g. Furnieren sowie einer PVC-Folie für die Mittelschicht ausgewählt. Für die Verklebung der verschiedenen Schichten kamen ein PVAc-basierter Klebstoff sowie ein thermoplastischer Klebefilm auf PU-Basis zum Einsatz.

Aufgrund dieser Materialauswahl waren den Herstellungsvariablen Pressdruck und Presstemperatur Grenzen gesetzt. Daher wurde die Presstemperatur auf $120^{\circ} \mathrm{C}$ und der Pressdruck auf $13,95 \mathrm{~N}^{*} \mathrm{~cm}^{-2}$ bei Verklebungen mittels thermoplastischem Klebefilm sowie $24,50 \mathrm{~N}^{*} \mathrm{~cm}^{-2}$ bei Verklebungen mittels PVAc festgelegt.

In einem ersten Schritt erfolgten Untersuchungen der Zugfestigkeit der eingesetzten PVC-Folie bei verschiedenen Ausgangsbedingungen. Die Ergebnisse der Zugfestigkeitsuntersuchungen lassen sich folgendermaßen zusammenfassen:

- Die Zugfestigkeit der PVC-Folie wurde nach DIN EN ISO 527-3 überprüft. 
Bei einem Vergleich der Zugfestigkeit der PVC-Folie in Abhängigkeit zu ihrer Herstellungsrichtung ließ sich feststellen, dass die Herstellungsrichtung einen signifikanten Einfluss auf die Zugfestigkeit hat. Die Differenz zwischen parallel und senkrecht zur Herstellungsrichtung genommener Probekörper lag bei 2,47 $\mathrm{N}^{*} \mathrm{~mm}^{-2}$. Die mit $14,91 \mathrm{~N}^{*} \mathrm{~mm}^{-2}$ höhere mittlere Festigkeit wurde parallel zur Herstellungsrichtung gemessen. Das gemittelte Gesamtergebnis der beiden Versuchsreihen (senkrecht und parallel) betrug 12,96 $\mathrm{N}^{*} \mathrm{~mm}^{-2}$.

- Um einen möglichen negativen Einfluss der Prozesstemperatur auf die Zugfestigkeitseigenschaften der PVC-Folie auszuschließen, wurde eine Gruppe Probekörper zunächst unter realen Pressbedingung (ohne Klebstoffeinsatz) vorbehandelt und im Anschluss mit unbehandelten Probekörpern verglichen.

Das Ergebnis ließ keine negativen Einflüsse der Presstemperatur auf die Zugfestigkeit der PVC-Folie erkennen. Tatsächlich lag der Festigkeitswert der thermisch behandelten Proben um 0,97 $\mathrm{N}^{*} \mathrm{~mm}^{-2}$ höher als bei den unbehandelten Vergleichsproben.

- Die wesentliche Funktion des in dieser Arbeit entwickelten Halbzeugs ist seine Faltbarkeit. Da an den Faltstellen (Faltnuten) die Furnierschicht entfernt wird, fehlt hier aussteifendes und festigendes Material. Zudem wird diese Faltnut bei Endprodukten häufig in einem angewinkelten Zustand sein.

Aus diesen Gründen wurden zusätzlich modifizierte Zugprüfungen jeweils in Winkel von $45^{\circ}$ und $90^{\circ}$ durchgeführt und den nach DIN EN ISO 527-3 geprüften Proben gegenübergestellt.

Erwartungsgemäß fielen die Ergebnisse der modifizierten Versuchsreihe niedriger aus als die der nach Norm geprüften. Die mittlere Zugfestigkeit belief sich bei einem Winkel von $45^{\circ}$ auf $10,95 \mathrm{~N}^{*} \mathrm{~mm}^{-2}$ und bei einem Winkel von $90^{\circ}$ auf $11,87 \mathrm{~N}^{*} \mathrm{~mm}^{-2}$. Der Vergleichswert der Normprüfung belief sich auf $12,96 \mathrm{~N}^{*} \mathrm{~mm}^{-2}$. In einem nächsten Schritt wurde anhand von Prüfungen der Abhebefestigkeit nach DIN EN 311 die optimale Presszeit als wesentliche Herstellungsvariable definiert. Weiterhin wurde mit diesen Untersuchungen die Qualität der Verklebung mittels thermoplastischen Klebefilms von Erlen- und Eichenfurnieren auf PVC-Folie bewertet und die Ergebnisse von Eiche und Erle einander gegenübergestellt. Zusätzlich erfolgte als weiterer Schritt der Vergleich der beiden Klebstoffsysteme (PVAc und Klebefilm) zur Eignung der Verklebung von Absperr- und Deckfurnier.

- Grundsätzlich ließ sich feststellen, dass der thermoplastische Klebefilm sehr gut zur Verklebung von Erlenfurnier auf PVC-Folie geeignet ist. Bei verschiedenen untersuchten Presszeiten von 60 s, 90 s, 120 s, 180 s und 240 s wurde als niedrigste mittlere Abhebefestigkeit ein Wert von $1,17 \mathrm{~N}^{*} \mathrm{~mm}^{-2}$ gemessen. Aus der Gegenüberstellung der Versuchsergebnisse konnte festgestellt werden, dass mit einer Presszeit von $120 \mathrm{~s}$ und einer mittleren Abhebefestigkeit von 1,71 $\mathrm{N}^{*} \mathrm{~mm}^{-2}$ die optimale Presszeit gefunden wurde. 
- Bei den Vergleichsuntersuchungen mit Eichenfurnier auf PVC-Folie fielen die Abhebefestigkeiten signifikant geringer aus als bei den analogen Untersuchungen mit Erlenfurnier. Die höchste gemessene mittlere Abhebefestigkeit lag bei $1,64 \mathrm{~N}^{*} \mathrm{~mm}^{-2}$. Um vergleichbare Werte mit Erlenfurnier $\mathrm{zu}$ erreichen, waren allerdings $180 \mathrm{~s}$ Presszeit nötig. Bei einer Presszeit von $120 \mathrm{~s}$ wurden bei Eichenfurnieren auf PVC-Folie 1,48 $\mathrm{N}^{*} \mathrm{~mm}^{-2}$ ermittelt.

Aufgrund der signifikant schlechteren Abhebefestigkeiten erfolgten keine weiteren Versuche von Verbünden mit Eichenfurnier durchgeführt.

- Die Verklebung von Absperr- und Deckfurnier geschah beim ursprünglichen Herstellungsprozess mittels PVAc-Leims. Da dieser Vorgang eine deutlich höhere Presszeit benötigt als eine Verklebung mit einem thermoplastischen Klebefilm wurde untersucht, ob der PVAc-Leim bei der Verklebung von Erlenfurnier durch den Klebefilm substituiert werden kann. Die Messung der Abhebefestigkeit erfolgte hierzu an Erlenfurnieren die sowohl mit PVAc-Leim als auch mittels thermoplastischem Klebefilms miteinander verklebt wurden.

Da es sich bei dem verwendeten PVAc-Leim um einen speziellen Furnierleim handelte, fielen die Ergebnisse der Abhebefestigkeit bei mit diesem System verklebten Erlenfurnieren erwartungsgemäß höher aus als bei den Vergleichsuntersuchungen mit thermoplastischem Klebefilm. Die mittlere Abhebefestigkeit der Versuchsreihe mit PVAc-Leim wurde mit 2,67 $\mathrm{N}^{*} \mathrm{~mm}^{-2}$, die der Versuchsreihe mit thermoplastischem Klebefilm mit 1,97 $\mathrm{N}^{*} \mathrm{~mm}^{-2}$ gemessen. Zusammenfassend lässt sich dennoch feststellen, dass der thermoplastische Klebefilm sehr wohl geeignet ist um Erlenfurniere miteinander zu verkleben. Dies bedeutet, dass bei der ausschließlichen Verwendung des Klebefilms bei der Halbzeugherstellung eine erhebliche Zeitersparnis möglich ist und sich dieses Verfahren somit als ökonomischer darstellt.

Die zu Projektbeginn als Zielgröße definierte Abhebefestigkeit von mindestens $0,80 \mathrm{~N}^{*} \mathrm{~mm}^{-2}$ konnte bei allen Materialkombinationen erreicht werden.

Um die Ursache für die signifikant schlechteren Abhebefestigkeiten von mittels thermoplastischem Klebefilm auf PVC-Folie verklebten Eichenfurnieren gegenüber des gleichen Verbundes aus Erlenfurnier festzustellen, wurde eine Untersuchung der beiden Furnierarten auf ihre chemisch-physikalischen Eigenschaften durchgeführt. Zunächst erfolgte eine Bestimmung der pH-Werte von Erlen- und Eichenfurnier mittels Kalt- und Heißwasserextraktion sowie durch eine sukzessive Extraktion mit verschiedenen Lösungsmitteln.

Im Anschluss wurde eine Messung der Oberflächentopographie beider Furnierarten durchgeführt, um einen möglichen Einfluss der Oberflächenrauigkeit auf die Qualität der Verklebung zu untersuchen.

- $\quad$ Die Bestimmung der pH-Werte und der Extraktstoffgehalte mittels Heiß- und Kaltwasserextraktion sowie sukzessiver Exktraktion ergab folgende Ergebnisse: 
Eichenfurnier ist mit einem in den wässrigen Auszügen der Kaltwasserextraktion gemessenen $\mathrm{pH}$-Wert von 3,85 saurer als Erlenfurnier mit einem $\mathrm{pH}$-Wert von 4,68 .

Sowohl bei der Heißwasserextraktion als auch bei der sukzessiven Extraktion mit Lösungsmitteln verschiedener Polarität fiel auf, dass besonders in den Lösungsmitteln in denen Tannine gelöst werden, Eichenfurniere einen deutlich höheren Extraktstoffgehalt als Erlenfurniere aufwiesen. Da Tannine für Beeinträchtigungen bei Verklebungen von Hölzern oder holzigen Partikeln verantwortlich gemacht werden, kann somit ein Einfluss des höheren Gehalts an Tanninen und/oder die stärkere Acidität von Eichenfurnieren für die geringeren Abhebefestigkeiten von Eichenfurnier auf PVC-Folie verantwortlich sein.

- Die Untersuchung der Oberflächenrauigkeit zeigte, dass Eichenfurniere mit Vertiefungen in der Oberfläche von bis $\mathrm{zu} 250 \mu \mathrm{m}$ deutlich rauer sind als Erlenfurniere mit Vertiefungen von bis zu $100 \mu \mathrm{m}$. Ein entscheidender Einfluss auf die Abhebefestigkeit durch die Struktur der Furnieroberflächen konnte nicht sicher nachgewiesen werden.

Im letzten Abschnitt des Promotionsvorhabens erfolgten mechanisch-technologische Untersuchungen des 5-schichtigen Gesamtverbundes aus Erlenfurnieren auf PVCFolie. In diesem Rahmen wurden die Biegefestigkeit, der Biege-E-Modul, die Dauerhaftigkeit der Faltnut sowie das Verhalten im starken Wechselklima überprüft. Diese Kenngrößen sind wesentlich für die Möglichkeit der Verwendung in verschiedenen Produktsegmenten (z. B. Kisten oder Möbel) und zur Bewertung der Haltbarkeit der Produkte.

- Die Prüfung der Biegefestigkeit nach DIN EN 310 des Halbzeugs aus Erlenfurnieren und PVC-Folie ergab folgende Ergebnisse. Bei Prüfung senkrecht zur Faserrichtung des Deckfurniers wurde eine Biegefestigkeit von 47,42 $\mathrm{N}^{*} \mathrm{~mm}^{-2}$ und ein Biege-EModul von 5,87 $\mathrm{kN}^{*} \mathrm{~mm}^{-2}$ gemessen. Parallel zur Faserrichtung des Deckfurniers ergab die Prüfung der Biegefestigkeit ein mittleres Ergebnis von $28,44 \mathrm{~N}^{*} \mathrm{~mm}^{-2}$. Der mittlere E-Modul wurde mit 2,01 N*mm-2 bestimmt.

Wie die Ergebnisse zeigen, handelt es sich bei Faltfurnier um einen Werkstoff mit hoher Biegefestigkeit. Die Ergebnisse fallen z. T. deutlich höher aus als die von im Bausektor verwendeten Holzwerkstoffen, wie OSB oder Spanplatten.

$\mathrm{Zu}$ Projektbeginn wurde für die Biegefestigkeit ein Wert von $20,00 \mathrm{~N}^{*} \mathrm{~mm}^{-2}$ als Zielgröße definiert. Dieser Wert wurde beim untersuchten Verbundwerkstoff übertroffen.

- Um die Dauerhaftigkeit eines Produktes aus Faltfurnier bewerten zu können, erfolgte ein Versuch, bei dem Probekörper, die mit einer Faltnut versehen waren, so häufig mittels einer Türprüfmaschine gefaltet wurden bis sich in der Mittelschicht Risse mit einer Größe von >20 mm feststellen ließen oder die PVC-Folie ganz versagte. 
Im Mittel wurden 4.441 Wiederholungen erreicht bis eines der definierten Abbruchkriterien eintraf. Dieser Wert scheint zunächst sehr positiv, muss aber mit Vorsicht bewertet werden, da die Streuung der einzelnen Ergebnisse recht stark ausfiel. $\mathrm{Zu}$ Beginn des Projektes wurde ein Wert von wenigsten $1.000 \mathrm{zu}$ erreichenden Faltvorgängen als Minimalanforderung bestimmt. Dieses Ziel konnte beim dem in dieser Arbeit entwickelten Werkstoff erreicht werden.

- Die Lagerung im starken Wechselklima bei einer Temperaturspanne von $5{ }^{\circ} \mathrm{C}$ bis $50{ }^{\circ} \mathrm{C}$ und einer Variation der Luftfeuchte von $10 \%$ bis $90 \%$ lieferte keine auffälligen Ergebnisse. Der Werkstoff blieb bis auf geringe Verwerfungen an seinen Kopfseiten formstabil. 


\section{Ausblick}

Wie sich im Laufe des Projektes herausstellte, ist der in dieser Arbeit entwickelte Werkstoff auch für viele weitere Anwendungen abseits des Verpackungssektors einsetzbar.

Einige Produkte (siehe Anhang) haben es mittlerweile zur Marktreife gebracht und stehen zum Verkauf. So sind derzeit beispielsweise Brillenetuis, Aktenmappen und Aktenkoffer beim Projektpartner zu erwerben. Als klarer Vorteil des Werkstoffes erwies sich beim Produktdesign der hohe Grad an Gestaltungs- und Personalisierungsmöglichkeiten.

Eine weitere Möglichkeit der Verwendung des Werkstoffes bietet die Anwendung im Möbelbau. So könnten beispielsweise platzsparende, weil faltbare, Tische für den Einsatz in kleinen Wohnräumen (z. B. Tiny Houses) oder Campingwagen zur Produktionsreife gebracht werden. Erste Vorversuche verliefen äußerst vielversprechend. Hier stellen jedoch die normativen Anforderungen an Möbel eine hohe Hürde dar.

Die Unsicherheiten aufgrund der hohen Abweichungen der Falzfestigkeit konnten wenige Tage vor Projektende durch den Einsatz einer neuen Mittelschicht behoben werden. Ein Spinnvlies aus Polyester mit ähnlicher Materialstärke wie die der PVCFolie, soll diese ersetzen. Die Gefahr des Aufspinnens einzelner Fäden durch den Fräskopf ist bei diesem Material nicht gegeben. Umfangreiche Materialuntersuchungen konnten hierzu noch nicht stattfinden, sind aber dringend anzuraten um Materialausschuss zu verhindern und die Werkstoffeigenschaften zu optimieren. Die ersten „Feldversuche“ bei Testern zur Zuverlässigkeit im Alltagsgebrauch verliefen bereits sehr positiv.

Aufgrund der verwendeten Materialien wie Furniere, Folien, Vliese und Klebefilme sollte über eine Effizienzsteigerung der Werkstoffproduktion nachgedacht werden. Diese Werkstoffe und die vergleichsweise kurze Presszeit/Produktionsdauer bieten hier noch einige Möglichkeiten das Halbzeug in größerem Umfang zu produzieren.

In der Zwischenzeit wurde durch den Projektpartner ein Gebrauchsmuster auf den Werkstoffverbund (DE202015003543U1) angemeldet. Der neue Werkstoffname lautet nun nicht mehr Faltfurnier sondern „Knick-Holz-Laminat“.

Da der Aspekt des Trends zum Umweltbewusstsein der Bevölkerung in den letzten Jahren noch einmal deutlich an Geschwindigkeit aufgenommen hat, wurde nach einem Lösungsansatz gesucht, die PVC-Schicht durch ein umweltverträglicheres Material zu ersetzen. 
Hierzu konnte ein Folgeprojekt initiiert werden, das sich mit der Verwendung von biobasierten Polymeren für die Mittelschicht beschäftigte. Die ersten Versuche verliefen vielversprechend, jedoch herrscht noch erheblicher Forschungsbedarf, um einen solchen Werkstoff zur Marktreife zu bringen. Eine Intensivierung der Forschungstätigkeit in diese Richtung ist äußerst wünschenswert. 


\section{Literaturverzeichnis}

Annies, Thomas (2010): Trocknen und Dämpfen. In: U. Lohmann (Hg.): Holz Handbuch. Unter Mitarbeit von Annies Thomas und Geerth-Adolph Peters. 7. völlig überarbeitete Auflage: DRW-Verl., S. 139-202.

Bablick, M. (2009): Holz und Holzwerkstoffe. Oberflächenbehandlung und Schutz. 1. Auflage. München: Deutsche Verlags-Anstalt.

Behets-Oschmann, L.; Knauf, M. (2010): Auf Furnier setzen. In: BM Bau- und Möbelschreiner (1), S. 30-31.

Bodig, J.; Jayne, B. (1982): Mechanics of Wood and Wood Composites. New York, Cincinnati, Toronto, London, Melbourne: Van Nostrand Reinhold Company. Brockmann, Walter; Geiß, Paul Ludwig; Klingen, Jürgen; Schröder, Bernhard (2005): Klebtechnik. Klebstoffe, Anwendungen und Verfahren. Weinheim: Wiley-VCH. Online verfügbar unter http://onlinelibrary.wiley.com/book/10.1002/3527605851.

Christiansen, Alfred W. (1991): How overdrying wood reduces its bonding to phenolformaldehyde adhesives. A critical review of the literature. Part II chemical reactions. In: Wood and Fiber Science 23 (1), S. 69-84.

Collano Adhesives AG (2011): Collano 46.002. Technisches Datenblatt. SempachStation/Schweiz.

consultic (2014a): Analyse der PVC Produktion, Verarbeitungs-, Abfall- und Verwertungsströme in Deutschland 2013. Unter Mitarbeit von C. Lindner. CONSULTIC MARKETING \& INDUSTRIEBERATUNG GMBH. Online verfügbar unter http://www.plasticseurope.de/Document/analyse-der-pvc-produktionverarbeitungs--abfall--und-verwertungsstrome-in-deutschland-2013.aspx?FolID=2, zuletzt geprüft am 28.07.2015.

Dadswell, H. E.; Hillis, W. E. (1962): Wood Extractives. and Their Significance to the Pulp and Paper Industrie. New York and London: Academic Press, S. 48. 
Diemert, J.; Hauk, J.; Stieneker, A.; Woidasky, J. (2005): Vinylpolymere. In: P. Eyerer, P. Elsner und T. Hirth (Hg.): Die Kunststoffe und ihre Eigenschaften. 6. Auflage. Berlin Heidelberg New York: Springer-Verlag, S. 606-674.

Dunky, Manfred (2002a): Bindemittel und Verleimung. Polyvinylacetatleime. In: Manfred Dunky und Peter Niemz (Hg.): Holzwerkstoffe und Leime. Technologie und Einflussfaktoren. Berlin Heidelberg New York: Springer-Verlag.

Dunky, Manfred (2002b): Einflussgrößen. Chemisches Verhalten des Holzes. In: Manfred Dunky und Peter Niemz (Hg.): Holzwerkstoffe und Leime. Technologie und Einflussfaktoren. Berlin Heidelberg New York: Springer-Verlag.

Dunky, Manfred; Niemz, Peter (2002): Holzwerkstoffe und Leime. Technologie und Einflussfaktoren. Berlin, Heidelberg: Springer-Verlag.

Euring, Markus (2008): Einsatz von Mediatoren bei der enzymatischen Aktivierung der fasereigenen Bindekräfte zur Herstellung von enzymgebundenen, bindemittelfreien Holzwerkstoffen. Dissertation. Georg-August-Universität Götttingen, Göttingen.

Eyerer, Peter (2005): Einführung in Polymer Engineering. In: Peter Eyerer, Peter Elsner und Thomas Hirth (Hg.): DOMININGHAUS Kunststoffe und ihre Eigenschaften. 6., neubearb. u. erw. Aufl. Berlin: Springer (VDI-Buch), S. 1-449.

Faix, Oskar (2012): Chemie des Holzes. In: Andre Wagenführ und Frieder Scholz (Hg.): Taschenbuch der Holztechnik. München: Carl Hanser verlag, S. 47-74.

Fengel, D.; Wegener, G. (2003): Wood. Chemistry, Ultrastructure, Reactions. Remagen: Kessel Verlag.

Franck, Adolf (2000): Kunststoff-Kompendium. Herstellung, Aufbau, Verarbeitung, Anwendung, Umweltverhalten und Eigenschaften der Thermoplaste, Polymerlegierungen, Elastomere und Duroplaste. 5., überarb. Aufl. Würzburg: Vogel (Vogel-Fachbuch).

Nationale Norm DIN 68330, 1976-08-00: Furniere.

Grosser, Dietger (2003): Das Holz der Schwarzerle. Eigenschaften und Verwendung. Hg. v. Bayerische Landesanstalt für Wald und Forstwirschaft (LWF). Freising (LWF Wissen, 42). 
Habenicht, Gerd (2002): Kleben. Grundlagen, Technologien, Anwendungen. 4. erweiterte Auflage. [Place of publication not identified]: Springer Berlin Heidelberg. Habenicht, Gerd (2009): Kleben. Grundlagen, Technologien, Anwendungen. 6. Aufl. Berlin: Springer.

Heermann, Paul; Herzog, Alois (1931): Mikroskopische und mechanisch-technische Textiluntersuchungen. Dritte vollständig neubearbeitete und erweiterte Auflage. Berlin, Heidelberg, s.l.: Springer Berlin Heidelberg.

Heissner GmbH (o. D.): Technisches Datenblatt. Teichfolie PVC 1 mm. Hg. v. Lauterbach Heissner GmbH.

Deutsche Norm DIN EN 310, August 1993: Holzwerkstoffe.

Deutsche Norm DIN EN 326-1, August 1994: Holzwerkstoffe - Probenahme, Zuschnitt und Überwachung.

Deutsche Norm DIN EN 311, August 2002: Holzwerkstoffe Abhebefestigkeit der Oberfläche.

IHB (2014): Deutsche Furnierbranche schrumpft weiter. Hg. v. IHB Das Netzwerk der Holzindustrie. Online verfügbar unter http://www.ihb.de/fordaq/news/Furnier_Furniergeschichten_Furniermarkt_Furnierind ustrie_39724.html, zuletzt geprüft am 16.07.2015.

IHB (2015): Interzum: Furnierbranche sieht Licht am Horizont. Hg. v. IHB Das Netzwerk der Holzindustrie. Onlinedokument. Online verfügbar unter http://www.ihb.de/fordaq/news/interzum_Ladenbau_Furnier_Formholz_41774.html, zuletzt geprüft am 22.07.2015.

Jackson, Paul (2012): Vom Entwurf zur Schachtel. Grundlagen des Verpackungsdesigns. 1. Auflage. Bern, Stuttgart, Wien: Haupt Verlag. Jaic, M.; Zivanovic, R.; Stevanovic-Janezic, T.; Dekanski A. (1996): Comparison of surface properties of Beech- and Oakwood as determined by ESCA method. In: HOLZ als Roh- und Werkstoff 54, S. 37-41. 
Kaiser, W. (2007): Kunststoffchemie für Ingenieure. Von der Synthese bis zur Anwendung. 2. Aufl. München: Hanser.

Kharazipour, Alireza (1996): Enzyme von Weißfäulepilzen als Grundlage für die Herstellung von Bindemitteln für Holzwerkstoffe. Frankfurt/Main: Sauerländer's Verlag (Schriften aus der Forstlichen Fakultät der Universität Göttingen und der Niedersächsischen Forstlichen Versuchsanstalt, 118).

KLEBCHEMIE M. G. Becker GmbH \& Co. KG (1999): Kleiberit Information. Funrierleim 322. Weingarten/Bd.

Knippers, Jan (2010): Atlas Kunststoffe und Membranen. Werkstoffe und Halbzeuge, Formfindung und Konstruktion. München: Institut für internationale ArchitekturDokumentation.

Koch, G.; Bauch, J.; Puls, J.; Schwab E.; Welling, J. (2000): Holzverfärbungen der Rotbuche (Fagus sylvatica [L.]) und Möglichkeiten vorbeugender Maßnahmen. In: Holz-Zentralblatt 126 (6), S. 74-75.

Kollmann, F. (Hg.) (1962): Furniere, Lagenhölzer und Tischlerplatten. Rohstoffe, Herstellung, Plankosten, Qualitätskontrolle usw. Unter Mitarbeit von Jürgen Häber. Berlin, Göttingen, Heidelberg: Springer-Verlag.

Kollmann, Franz (1955): Technologie des Holzes und der Holzwerkstoffe. 2 Bände.

Berlin, Göttingen, Heidelberg: Springer-Verlag (1).

Deutsche Norm DIN EN ISO 527-1, Juni 2012: Kunststoffe - Bestimmung der Zugeigenschaften.

Deutsche Norm DIN EN ISO 527-3, Juli 2003: Kunststoffe - Bestimmung der Zugeigenschaften.

Lohmann, U. (Hg.) (2003): Holz Lexikon. Band 1 A-K. 4. Auflage. 2 Bände. LeinfeldenEchterdingen: DRW-Verlag Weinbrenner GmbH \& Co.

Lohmann, U. (Hg.) (2010): Holz Handbuch. Unter Mitarbeit von Annies Thomas und Geerth-Adolph Peters. 7. völlig überarbeitete Auflage: DRW-Verl.

Müller, Rüdiger (1984): Herstellung von wasserfesten und beständigen Fenster- und Türelementen. In: Rudolf Hinterwaldner (Hg.): KLEBEN UND LEIMEN RUND UM'S 
HOLZ. Vortragsreihe des 5. Internationalen Klebtechnik-Seminars am Lehrinstitut der Holzwirtschaft und Kunststofftechnik e. V. D-8200 Rosenheim. Sonderdokumentation des KLEBSTOFF-DOKUMENTUMS. München: Hinterwaldner-Verlag, S. 45-53.

Niemz, Peter (2002a): Grundlagen. Eigenschaften ausgewählter Holzwerkstoffe. In: Manfred Dunky und Peter Niemz (Hg.): Holzwerkstoffe und Leime. Technologie und Einflussfaktoren. Berlin Heidelberg New York: Springer-Verlag.

Niemz, Peter (2002b): Grundlagen. Übersicht zu den Holzwerkstoffen. In: Manfred Dunky und Peter Niemz (Hg.): Holzwerkstoffe und Leime. Technologie und Einflussfaktoren. Berlin Heidelberg New York: Springer-Verlag.

Niemz, Peter; Sonderegger, Walter (2017): Holzphysik. Physik des Holzes und der Holzwerkstoffe. München: Fachbuchverlag Leipzig im Carl Hanser Verlag.

Otto GmbH \& Co KG (2013): Lebensqualität. Konsumethik zwischen persönlichem Vorteil und sozialer Verantwortung. Otto Group Trendstudie 2013 4. Studie zum ethischen Konsum. Hamburg, 2013, S. 7-9. Online verfügbar unter http://www.ottogroup.com/trendstudie, zuletzt geprüft am 15.07.2015.

Popper, Rudolf (1974): Die Einflüsse der Holzextraktstoffe auf das technologische Verhalten von Holz, insbesondere in bezug auf die Verleimung. ETH-Zürich. Zürich (Research Collection). Online verfügbar unter https://doi.org/10.3929/ethz-a-006121556, zuletzt geprüft am 26.03.2020.

Retting, W. (1975): Orientierung, Orientierbarkeit und mechanische Eigenschaften von thermoplastischen Kunststoffen. In: Colloid \& Polymer Sci. 253 (10), S. 852-874.

Ritter, Nina (2011): Entwicklung leichter, dreischichtiger Spanplatten aus einer Kombination der nachwachsenden Rohstoffe Mais und Holz. Dissertation. GeorgAugust-Universität Götttingen, Göttingen.

Roffael, E.; Rauch, W. (1974): Extraktstoffe in Eiche und ihr Einfluß auf die Verleimbarkeit mit alkalischen Phenol-Fomaldehydharzen. In: HOLZ als Roh- und Werkstoff (32), S. 182-187. 
Roffael, E.; Schäfer, M. (2002): Bedeutung der Extraktstoffe des Holzes in biologischer, chemischer und technologischer Hinsicht. Vorlesungsskript des Institutes für Holzbiologie und Holztechnologie, Georg-August-Universität Göttingen.

Sachsse, Hanno (1984): Einheimische Nutzhölzer und ihre Bestimmung nach makroskopischen Merkmalen. Hamburg: Parey (Pareys Studientexte, Nr. 44).

Sandermann, W.; Brendel, M. (1956): Die "zementvergiftende" Wirkung von Holzinhaltsstoffen und ihre Abhängigkeit von der chemischen Konstiution. In: HOLZ als Roh- und Werkstoff 14 (8), S. 307-313.

Schneider, Adolf (1966): Der Einfluß von Wärmebhandlungen im Temperaturbereich bis $200{ }^{\circ} \mathrm{C}$ und Wasserlagerung bis $100{ }^{\circ} \mathrm{C}$ auf den spektralen Remissionsgrad verschiedener Hölzer. In: HOLZ als Roh- und Werkstoff 24 (11), S. 542-546.

Schrott, P. (2010): Eine generelle Marktanalyse für Furniere. Eine Branche im Wandel. Saarbrücken: VDM Verlag Dr. Müller Aktiengesellschaft \& Co. KG.

Seintsch, B. (2013): Cluster Forst und Holz nach neuer Wirtschaftszweigklassifikation Tabellen für das Bundesgebiet und die Länder 2000 bis 2011. Thünen Working Paper 5. Braunschweig, S. 40-41. Online verfügbar unter http://literatur.ti.bund.de/digbib_extern/bitv/dn052186.pdf, zuletzt geprüft am 15.07.2015.

Simona (2016): technisches Handbuch. Simona Kunststoffe. Simona AG. Kirn. Online verfügbar unter https://www.simona.de/fileadmin/user_upload/Medien/Mediacenter/Technische_Infor mationen/technisches.Handbuch_SIMONA_Kunststoffe.pdf, zuletzt geprüft am 24.03.2020.

Soiné, H. (1995): Holzwerkstoffe. Herstellung und Verarbeitung ; Platten, Beschichtungsstoffe, Formteile, Türen, Möbel. Leinfelden-Echterdingen: DRW-Verl. Steffens, Henning; Bombosch, Firedbert (2007): Holzwirtschaft - Jobmotor im ländlichen Raum - Aspekte zur Holzmobilisierung. Cluster Forst und Holz in Thüringen aus volkswirtschaftlicher Sicht. In: Holzzentralblatt, 04.07.2007 (Nr. 27), S. $757-758$. 
Tsoumis, George (2009): Science ans Technology of wood. Structure, Properties, Utilization. Reprint of the edition from 1991. Remagen-Oberwinter: Verlag Kessel. VDM (2017): Die Lage der Furnierwirtschaft. Online verfügbar unter https://www.moebelfertigung.com/news/23683, zuletzt aktualisiert am 12.12.2017, zuletzt geprüft am 06.03.2020.

Weißmann, G. (1984): Zusammensetzung der wässrigen Rindenextrakte von Picea abies Karst. im Verlauf einer Vegetationsperiode. In: Holzforschung 38 (1), S. 7-10. Werndl, Peter (2014): Untersuchungen ausgewählter Materialeigenschaften eines neuartigen, faltbaren Verbundwerkstoffes. Bachelorarbeit. Hochschule Rosenheim, Rosenheim. Fakultät für Holztechnik.

Werndl, Peter (2016): Entwicklung eines biobasierten, faltbaren Verbundwerkstoffes unter Berücksichtigung spezieller technologischer Materialeigenschaften. Masterarbeit. Hochschule Rosenheim, Rosenheim. Fakultät für Holztechnik.

Zeppenfeld, Günter; Grunwald, Dirk (2005): Klebstoffe in der Holz- und Möbelindustrie. 2., überarb. u. erw. Aufl. Leinfelden-Echterdingen: DRW-Verl. Weinbrenner. 


\section{Anhang}

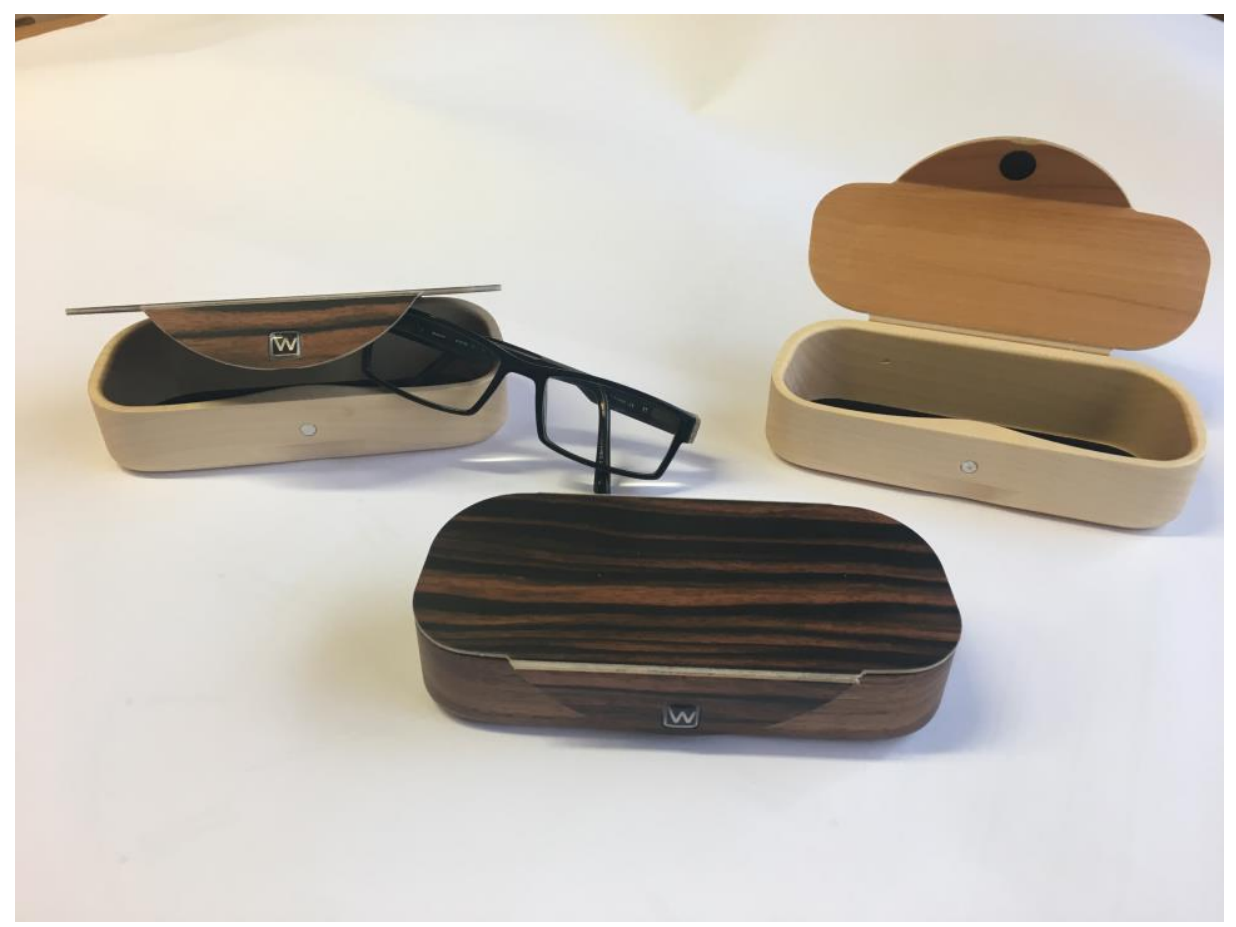

Anhang 8-1: Brillenentuis mit Deckeln aus Faltfurnier (Foto: Wolfgang Wochner).

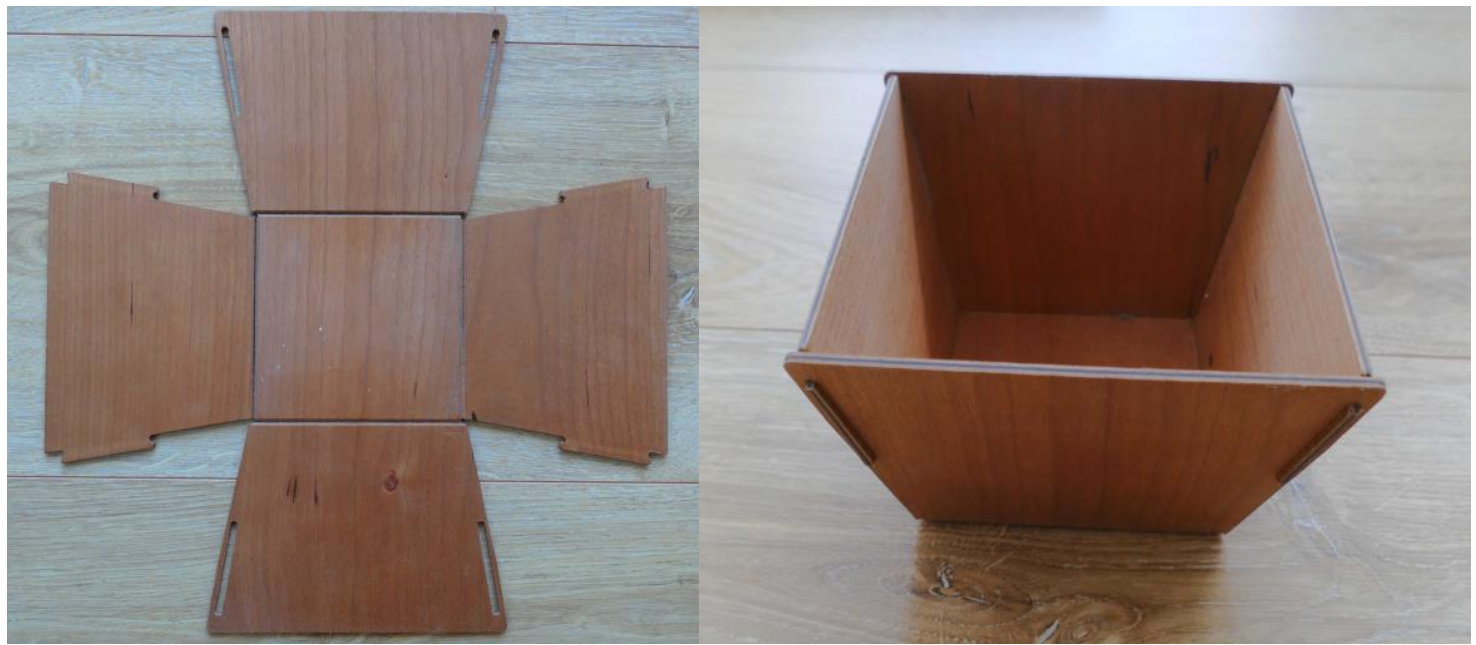

Anhang 8-2 Schale aus Faltfurnier, links geöffnet, rechts gefaltet. 


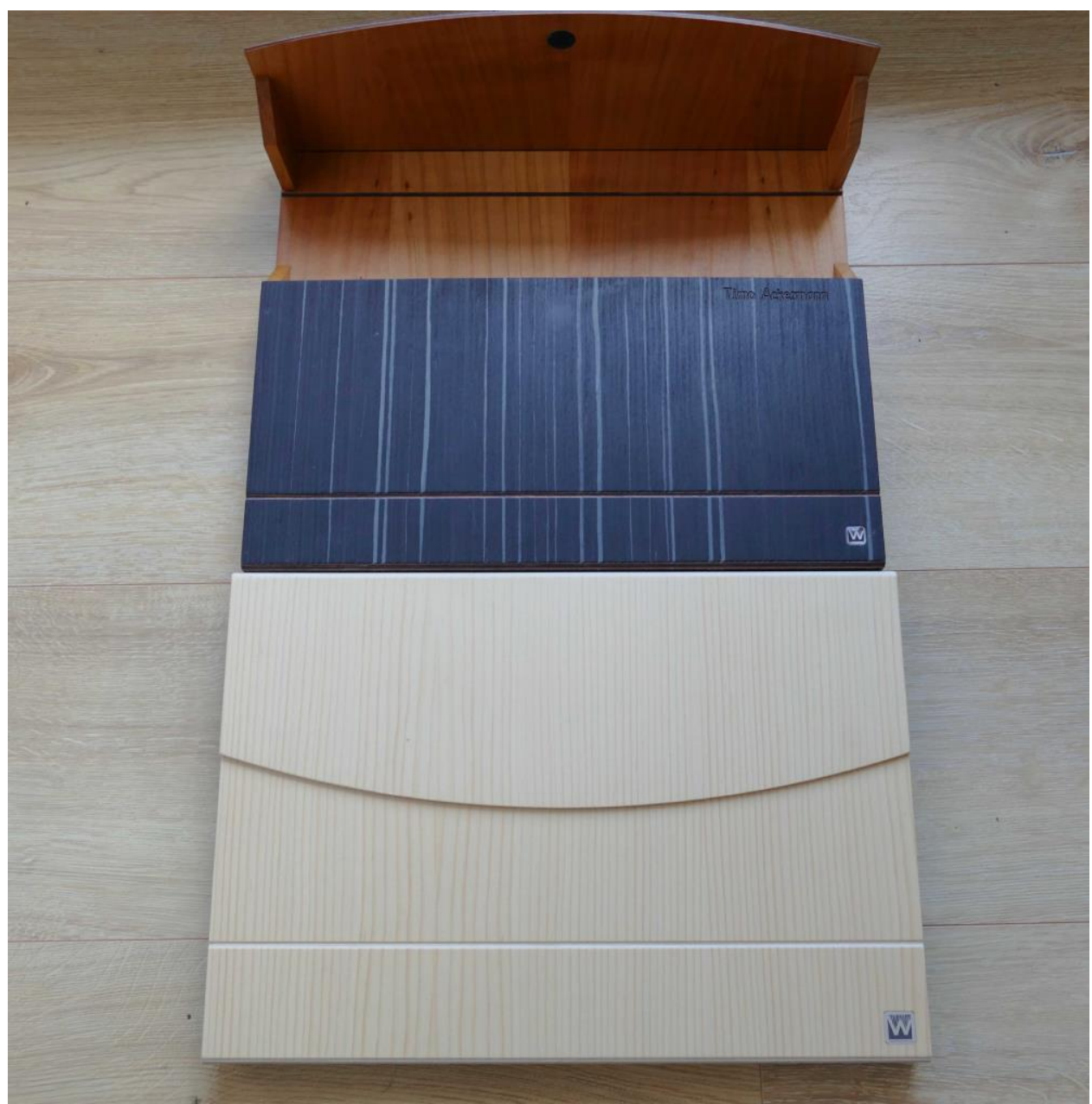

Anhang 8-3: Aktenmappen aus Faltfurnier.

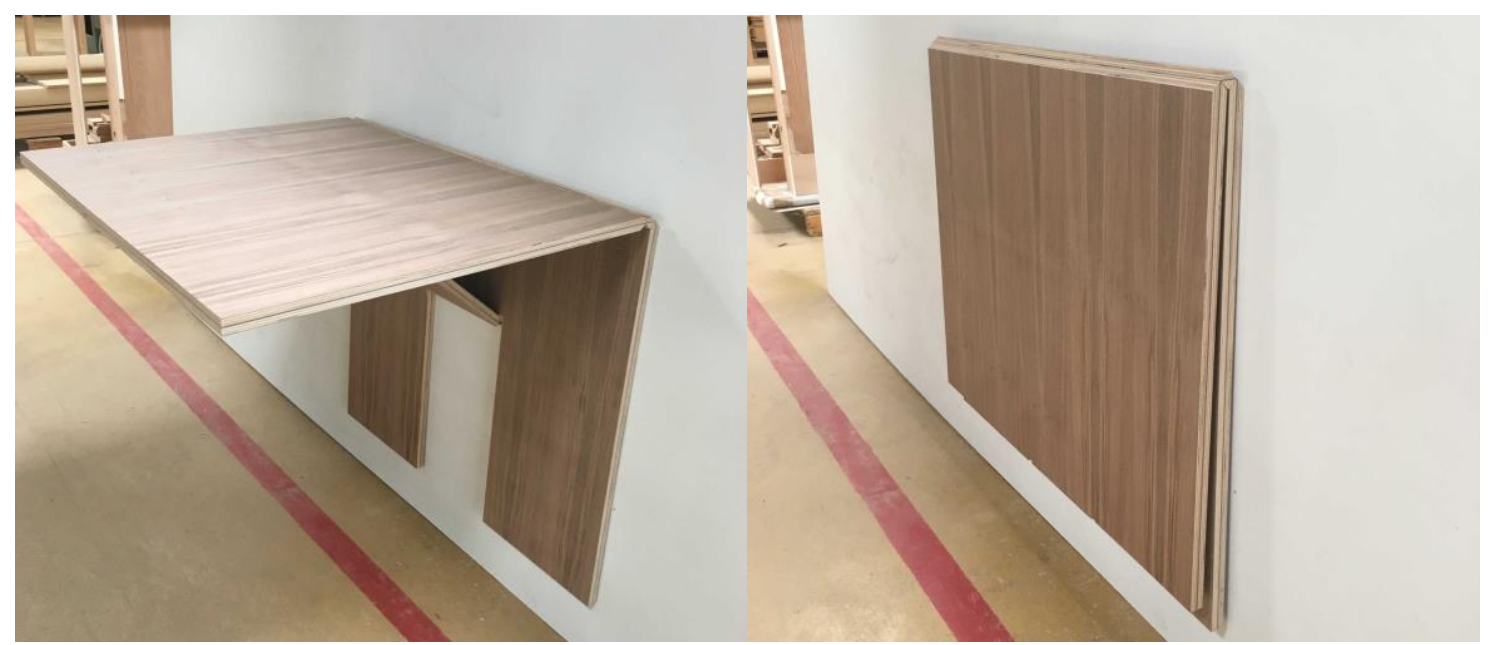

Anhang 8-4: Prototyp eines Klapptisches zur Wandbefestigung aus Faltfurnier, links aufgebaut, rechts zusammengeklappt (Foto: Wolfgang Wochner). 


\section{Eidesstattliche Erklärung}

Hiermit versichere ich, dass ich die vorliegende Dissertation selbständig verfasst habe und keine anderen als die angegebenen Quellen und Hilfsmittel benutzt habe.

Die Dissertation wurde in keinem anderen Prüfungsverfahren vorgelegt.

Ich habe den angestrebten Doktorgrad noch nicht erworben und bin nicht in einem früheren Promotionsverfahren für den angestrebten Doktorgrad gescheitert.

(Timo Ackermann)

Münster, 31. März 2020 